\title{
OPTIMAL NEUTRON SOURCE \& BEAM SHAPING ASSEMBLY FOR BORON NEUTRON CAPTURE THERAPY
}

\author{
SUMMARY REPORT
}

NEER Award Number

DE-FG07-98ID13642
J. Vujic (PI) E. Greenspan (Co-PI) W.E. Kastenberg (Co-PI)
Y. Karni and D. Regev
Department of Nuclear Engineering
University of California
Berkeley, CA 94720-1730
(510) 643-8085
vujic@nuc.berkeley.edu
gehud@,nuc.berkeley.edu
kastenbe@,nuc.berkeley.edu

with contributions from

J.M. Verbeke, K.N. Leung

D. Chivers, S. Guess, L. Kim, W. Waldron, Y. Zhu

Department of Nuclear Engineering

University of California

Berkeley, CA 94720-1730

Date published: April 2003 


\section{ACKNOWLEDGMENT}

This work was supported by the DOE NEER Program under contract No. DE-FG07-98ID13642 


\begin{abstract}
There were three objectives to this project:

1. The development of the 2-D SWAN code for the optimization of the nuclear design of facilities for medical applications of radiation, radiation shields, blankets of acceleratordriven systems, fusion facilities, etc.
\end{abstract}

2. Identification of the maximum beam quality that can be obtained for Boron Neutron Capture Therapy (BNCT) from different reactor-, and accelerator-based neutron sources. The optimal beam-shaping assembly (BSA) design for each neutron source was also to be obtained.

3. Feasibility assessment of a new neutron source for BNCT and other medical and industrial applications. This source consists of a state-of-the-art proton or deuteron accelerator driving an inherently safe, proliferation resistant, small subcritical fission assembly.

The 2-D SWAN code was successfully developed by replacement of the 1-D ANISN code for the solution of the transport equation by the 2-D DORT code and by making modifications to the SWIF module of the 1-D SWAN code. However, we found it difficult to get DORT to properly converge for the type of problems we were to consider in order to accomplish the second and third objectives of this project. Particularly problematic was the convergence of the adjoint equation. Not succeeding to resolve the DORT convergence problem, we developed a second version of the 2-D SWAN code in which DORT is replaced by TWODANT - another twodimensional discrete ordinates code in common use. The two versions of the 2-D SWAN codes are described in this report along with their validity tests and sample problems. In fact this report can serve as a draft of the users' manual for the newly developed 2-D SWAN codes.

As the TWODANT version of the 2-D SWAN code became operational only in 2002, we could not use the 2-D SWAN code for the optimization studies called for the second and third part of this project. Nevertheless, a study of the maximum attainable beam quality and an optimization of the BSA for two high-energy neutron sources was performed in parallel using 3-D Monte Carlo codes. A summary of his study is included in this report. It defines the optimal neutron energy for the treatment of deep and shallow seated brain tumors and comes up with an optimal BSA design for a D-D and a D-T fusion neutron sources. The findings of this study confirm our assertion that the beam quality that can be obtained from a high-energy neutron source is comparable to the beam quality that can be obtained using a low-energy neutron source. This assertion of ours, first published in 1995, was based on studies using the 1-D SWAN code.

The feasibility of using a subcritical fission assembly for amplifying the neutron current coming out from an accelerator neutron source was recently completed at UC Berkeley. This preliminary feasibility study considered a very compact $(26 \mathrm{~cm}$ in diameter, $21 \mathrm{~cm}$ long) cylindrical D-D neutron source coupled with a compact ( $80 \mathrm{~cm}$ in diameter, $14 \mathrm{~cm}$ thick) low-enriched uranium water-cooled subcritical neutron multiplier to provide a compact BNCT facility that is passively safe and economical. The fission multiplier amplifies the neutron current by a factor of 30 thus making the compact D-D neutron source useable for BNCT and other applications. However, a more thorough study is needed before a sound conclusion can be drawn on the desirability of 
developing commercial compact and possible mobile intense neutron irradiation-facilities that will be affordable to hospitals, research laboratories, industry and universities. 


\section{TABLE OF CONTENTS}

ABSTRACT

iii

INTRODUCTION

PART I: Development of a 2-D Optimization Code SWAN

1. INTRODUCTION

2. TYPE OF PROBLEMS 2D-SWAN CAN ADDRESS

4

3. THEORETICAL FOUNDATION

3.1 MATERIAL EFFECTIVENESS FUNCTIONS

3.2 OPTIMALITY CONDITION

3.3 DISCRETE MESH IMPLEMENTATION

3.4 VARIATION OF COMPOSITION

3.5 CONVERGENCE CONDITIONS

4. THE OPTIMIZATION PROCESS

5. 2D-SWAN STRUCTURE

5.1 OVERALL STRUCTURE

5.2 THE SWIF OPTIMIZATION MODULE

5.3 GIP AND DORT MODIFICATIONS

GIP

5.3 .2

DORT

5.4 I/O FILES

\section{4 .1}

GIP

5.4 .2

DORT

5.4 .3

SWIF

6. INPUT DATA DESCRIPTION 19

6.1 DORIF DRIVER 20

6.2 DORT INPUT SPECIFIC FOR "X” CASE 20

6.3 DORT INPUT COMMON FOR ALL CASES 23

6.4 GIP 26

6.5 DORT FLUX GUESS INPUT FOR THE THREE CASES 27

6.6 DORT FLUX INPUT AND OUTPUT UNITS 
6.7 SWIF INPUT 29

6.8 SWIF COMPOSITION INPUT 32

6.9 DORIF COMPOSITION INPUT 33

6.10 INPUT SAMPLE 34

6.11 OUTPUT SAMPLE 47

$\begin{array}{ll}7 . & \text { VALIDITY TESTS }\end{array}$

8. DORT CONVERGENCE PROBLEMS 58

8.1 INTRODUCTION 58

8.2 TEST PROBLEM DESCRIPTION 58

8.3 CONVERGENCE PROBLEMS 61

$\begin{array}{ll}\text { REFERENCES } & 69\end{array}$

PART II: Identification of the Maximum Attainable Beam Quality for BNCT 72

PART III: Feasibility Assessment of an Accelerator-Driven Subcritical Fission Neutron Source

APPENDIX A: UC BERKELEY STUDIES RELATED TO MAXIMUM ATTAINABLE BEAM QUALITY AND OPTIMAL BEAM SHAPING ASSEMBLY DESIGNS

75

APPENDIX B: FEASIBILITY ASSESSMENT OF AN ACCELERATOR-DRIVEN SUBCRITICAL FISSION NEUTRON SOURCE

ATTACHMENT:

J.M. Verbeke, J. Vujic, and K.N. Leung, "Neutron Beam Optimization for BNCT using the D-D and D-T High-Energy Neutron Sources," Nucl. Technol. 129 (2), 257-278 (2000). 


\section{INTRODUCTION}

There were three primary goals to this project:

(1) Development of a 2-D SWAN. The objective is to develop a first-of-its-kind general-purpose two-dimensional code for the optimization of source-driven systems. Examples of type of systems the 2-D SWAN is to be applicable to include facilities for medical applications, radiation shields, and blankets of accelerator-driven systems and of fusion facilities.

(2) Identification of the maximum attainable BNCT beam quality. The intention was to use the 2-D SWAN to identify the maximum beam quality for Boron Neutron Capture Therapy (BNCT) that can be obtained from different reactor-, and accelerator-based neutron sources. The optimal beam-shaping assembly (BSA) design for each neutron source was to be obtained.

(3) Feasibility Assessment of an Accelerator-Driven Subcritical Neutron Source. This source, intended for BNCT and other medical and industrial applications, is to consist of a state-of-the-art proton (or deuteron) accelerator driving a small, inherently safe, proliferation resistant subcritical fission assembly. The 2-D SWAN code was to be used for finding the optimal design of the subcritical neutron multiplier as well as of the BSA.

The first goal was formally accomplished as planned. However, we found it difficult to get DORT to properly converge for the type of problems we were to consider in order to accomplish the second and third goals. Particularly problematic was the convergence of the adjoint equation. Hence, we devoted all the time originally allocated for the second and third goals to search how to make DORT to converge.

Eventually we decided to replace DORT by TWODANT - another two-dimensional discrete ordinates code in common use. Thus we have developed another version of 2-D SWAN with TWODANT as the module for solving the transport equation.

This report describes the two two-dimensional optimization codes we developed and their validation. The focus is on the DORT version of the 2-D SWAN as it is with this code that we have invested most of our effort. The types of problems the SWAN codes can address are described in Section 2. The methodology used by the SWAN codes to search for the optimal system composition is summarized in Section 3. The SWAN optimization process is described in Section 4 and the 2D-SWAN code structure is outlined in Section 5. Input data description for the new 2D-SWAN code is described in Section 6. The validity of the 2-D SWAN is then addressed in Section 7 by comparing its predictions to results obtained using the well-established 1-D SWAN code. This validity test is done for design optimization of a simplified beamshaping-assembly (BSA) for Boron Neutron Capture Therapy (BNCT). Convergence problems encountered with the 2D-SWAN code are discussed in Section 8. 
Also included in this report is a summary of companion studies we have performed that address the subject of Part II and of Part III of this project and fulfill a significant part of their objective. These summaries are incorporated as Appendices of this report. 


\section{PART I}

\section{Development of a 2-D Optimization Code SWAN}

\section{INTRODUCTION}

The 2-D SWAN was to be developed on the basis of the one-dimensional optimization code SWAN originally developed and extensively applied ${ }^{1-39}$ by one of the co-PI's of this project. The original SWAN was able to address performance parameters of the form of linear flux functionals in fission-free systems. Since then the SWAN capability was extended; nowadays it can also be applied to (1) fissionable systems ${ }^{30-34}$; (2) performance parameters of the form of ratio of linear flux functionals ${ }^{27,28,32,34-39}$; and (3) critical systems, considering reactivity as the performance parameter ${ }^{30,31,33}$.

SWAN is composed of two major modules ${ }^{2,33}$ : (1) The one-dimensional discrete ordinates code ANISN for calculating the neutron flux and one or more adjoint functions across the system, and (2) The code SWIF that uses the flux and adjoint functions to calculate material "effectiveness functions" and uses these functions to guide an iterative search of the optimal system composition. Details will be given in the next section.

The approach proposed for the development of the 2-D SWAN is, basically, to replace the 1-D transport code ANISN by its 2-D "brother" DORT, and to modify the SWIF module to handle 2D EF's and 2-D material densities. It was anticipated that "having the experience we have in developing and successfully applying the 1-D SWAN, this modification is, in principle, straight forward. Nevertheless, there are few technical challenges to overcome." (This quotation is from the proposal). 


\section{TYPE OF PROBLEMS 2-D SWAN CAN ADDRESS}

The SWAN codes differ from conventional nuclear design codes in, essentially, a couple of ways: (a) They calculate "material effectiveness functions" (MEF) in addition to the flux, eigenvalue, and reaction rate distributions. (b) They use these MEF distributions to guide an automated search of the optimal system composition. The definition of the MEF is given in Section 3 and the search algorithm is described in Section 4.

The 2-D SWAN code can address the following type of optimization problems: find those constituents and their concentration across the system that will maximize or minimize a performance parameter of interest. The performance parameters that can be addressed with the code are of either one of the following forms:

(a) Linear flux functionals - such as reaction rates (for example: dose rate), or

(b) Ratio of linear flux functionals (e.g., ratio of dose-equivalent delivered to the tumor to maximum (or average) dose-equivalent delivered to the normal tissue - for BNCT).

(c) the maximum $k_{\text {eff }}$ for a given mass of fissile material of a given composition that can be configured when in combination with specified constituents (such as moderating and reflecting materials); and

(d) the minimum critical mass of a given type of fissile material when in combination with specified other materials and geometry.

The latter two apply only to multiplying systems. A constraint can be imposed on the optimization problem. 


\section{THEORETICAL FOUNDATION}

\subsection{MATERIAL EFFECTIVENESS FUNCTIONS}

2D-SWAN handles three different types of MEF's:

(a) EF, the Effectiveness Function, is the most basic MEF. The EF of material $\mathrm{i}$ in zone $\mathrm{z}$ with respect to performance parameter $\mathrm{P}$ expresses the effect, on $\mathrm{P}$, of an addition of a unit volume of material i in zone $\mathrm{z}$. EF is calculated using first-order perturbation theory formulation (See Sect. 3).

(b) EVREF, the Equal Volume Replacement Effectiveness Function, is the most widely used MEF. The EVREF of material $\mathrm{i}$ in zone $\mathrm{z}$ corresponding to a performance parameter $\mathrm{P}$ is the change in $\mathrm{P}$ due to the replacement of a cubic centimeter of reference material $\mathrm{R}$ in $\mathrm{z}$ by the same volume of material i. EVREF is calculated from the EF values of materials $i$ and $\mathrm{R}$ supplemented by material density data.

(c) ECREF, the Equal Cost Replacement Effectiveness Function, of material i in zone $\mathrm{z}$ corresponding to a performance parameter $\mathrm{P}$ is the change in $\mathrm{P}$ due to the replacement of a cubic centimeter of reference material $\mathrm{R}$ in $\mathrm{z}$ by that volume of material $\mathrm{i}$ that will leave the system cost unchanged. ECREF is calculated from the EF values of materials $i$ and $R$ supplemented by material cost data.

The MEF data provides the following information on the status of the specified system:

(a) Proximity of the present system to the optimum configuration.

(b) The system constituents which would most efficiently meet the optimization goals.

(c) Changes to the space-dependent concentration of these constituents needed to approach the optimal system (this information is used by SWAN to carry out the system optimization).

(d) Identification of alternate constituents considered to be more promising than one of the system constituents (this information is useful for determining if it is possible to get an even more optimal performance by replacing part of at least one of the system constituent by another material not incorporated in the system description).

The EF distributions are calculated using first order perturbation theory. Consider, first, performance parameters of the form of reaction rates. For problems in which the response function (usually proportional to a cross section) of the performance parameter to be maximized (or minimized) does not change with the change in composition, the values for $\mathrm{E}_{\mathrm{i}}(\mathrm{z})$ is calculated using the following expressions:

$\mathrm{E}_{\mathrm{i}}(\mathrm{z})=-\mathrm{AC}_{\mathrm{i}}(\mathrm{z})+\mathrm{BC}_{\mathrm{i}}(\mathrm{z})+\mathrm{FC}_{\mathrm{i}}(\mathrm{z})$ 
in which

$\mathrm{AC}_{\mathrm{i}}(\mathrm{z})=\int \mathrm{d} \underline{\mathrm{r}} \int \mathrm{dE} \int \mathrm{d} \underline{\Omega}\left[\Phi^{+}(\underline{\mathrm{r}}, \mathrm{E}, \underline{\Omega}) \Sigma_{\mathrm{t}, \mathrm{i}}(\mathrm{E}) \Phi(\underline{\mathrm{r}}, \mathrm{E}, \underline{\Omega})\right]$,

$\mathrm{BC}_{\mathrm{i}}(\mathrm{z})=\int \mathrm{d} \underline{\mathrm{r}} \int \mathrm{dE} \int \mathrm{d} \underline{\Omega} \int \mathrm{d} \mathrm{E}^{\prime} \int \mathrm{d} \underline{\Omega^{\prime}}\left[\Phi^{+}\left(\underline{\mathrm{r}}, \mathrm{E}^{\prime}, \underline{\Omega^{\prime}}\right)\right.$

$\left.\Sigma_{\mathrm{s}, \mathrm{i}}\left(\mathrm{E}->\mathrm{E}^{\prime} ; \Omega->\underline{\Omega}\right) \Phi(\underline{\mathrm{r}}, \mathrm{E}, \underline{\Omega})\right]$,

$\mathrm{FC}_{\mathrm{i}}(\mathrm{z})=\int \mathrm{dr} \int \mathrm{d} \mathrm{E}^{\prime}\left[\chi_{\mathrm{i}}\left(\mathrm{E}^{\prime}\right) \Phi^{+}\left(\underline{\mathrm{r}}, \mathrm{E}^{\prime}\right)\right] \int \mathrm{dE}\left[v_{\mathrm{i}} \Sigma_{\mathrm{f}, \mathrm{i}}(\mathrm{E}) \Phi(\underline{\mathrm{r}}, \mathrm{E})\right]$,

where $\int \mathrm{d}$ r denotes integration over the volume of zone $\mathrm{z}$,

$\Phi(\underline{\mathrm{r}}, \mathrm{E})=\int \mathrm{d} \underline{\Omega} \Phi(\underline{\mathrm{r}}, \mathrm{E}, \underline{\Omega})$, and

$\Phi^{+}(\underline{r}, \mathrm{E})=\int \mathrm{d} \underline{\Omega} \Phi^{+}(\underline{r}, \mathrm{E}, \underline{\Omega}) / 4 \pi$.

The EF distributions are then used to calculate the EVREF distributions as follows:

$\mathrm{EVREF}_{\mathrm{i}, \mathrm{R}}(\mathrm{z})=-\mathrm{E}_{\mathrm{R}}(\mathrm{z})+\mathrm{E}_{\mathrm{i}}(\mathrm{z})$.

Consider, next, a performance parameter of the form of a ratio of reaction rates, to be denoted as $\mathrm{N} / \mathrm{D}$. The EF used for such a performance parameter is

$\mathrm{EF}(\mathrm{N} / \mathrm{D})=[\mathrm{N} / \mathrm{D}][\mathrm{EF}(\mathrm{N}) / \mathrm{N}-\mathrm{EF}(\mathrm{D}) / \mathrm{D}]$

where $\mathrm{EF}(\mathrm{N})$ and $\mathrm{EF}(\mathrm{D})$ are $\mathrm{E}_{\mathrm{i}}(\mathrm{z})$ of Equation (1) when applied to, respectively, the numerator, $\mathrm{N}$, and the denominator, $\mathrm{D}$, of the performance parameter of interest. The EVREF for this performance parameter is of the same form as of Equation (7).

\subsection{OPTIMALITY CONDITION}

The criterion for the optimum system is the uniformity of the $\operatorname{EVREF}_{\mathrm{i}, \mathrm{R}}(\mathrm{z})$ of the constituent materials that are the optimization variables. This condition implies that, at the optimum, any permissible infinitesimal change in the concentration of the variable constituents should leave the performance parameter to be maximized or minimized unchanged, provided that

$$
0 \leq{ }^{\min } \psi_{\mathrm{i}}^{\mathrm{n}}(\mathrm{z}) \leq \psi_{\mathrm{i}}^{\mathrm{n}}(\mathrm{z}) \leq{ }^{\max } \psi_{\mathrm{i}}^{\mathrm{n}}(\mathrm{z}) \leq 1.0
$$


where $\psi_{\mathrm{i}}^{\mathrm{n}}(\mathrm{z})$ is the volume fraction of constituent $\mathrm{i}$ in zone $\mathrm{z}$ and iteration $\mathrm{n} ;{ }^{\min } \psi_{\mathrm{i}}^{\mathrm{n}}(\mathrm{z})$ is the minimum permissible volume fraction (usually 0) and ${ }^{\max } \psi_{\mathrm{i}}^{\mathrm{n}}(\mathrm{z})$ is the maximum permissible volume fraction. Material conservation requires that

$\sum_{\mathrm{i}} \psi_{\mathrm{i}}^{\mathrm{n}}(\mathrm{z})=$ constant $\leq 1.0$

where the summation is over all the $\mathrm{i}$ constituents of variable concentration. If the summation is smaller than unity, $(1.0$ - constant $)$ fraction of the volume of zone $\mathrm{z}$ is allocated for materials of fixed concentration. Examples for such materials are structural or moderator materials. The volume fraction is used as a measure of concentration since 2D-SWAN is set to work with macroscopic cross sections of the materials that are either incorporated in the system or are candidate constituents. SWAN implements this condition by requiring that the difference between the maximum and minimum value of the $\operatorname{EVREF}_{\mathrm{i}}(\mathrm{z})$ of all the material variables in all the ones will be smaller than a user-specified small number, $\varepsilon$. If and where a given material reaches an upper bound restraint (usually, it occupies $100 \%$ of the given zone volume), its $\operatorname{EVREF}_{\mathrm{i}}(\mathrm{z})$ is to be equal to or smaller than the unrestrained value of $\operatorname{EVREF}_{\mathrm{i}}(\mathrm{z})$. The opposite situation occurs in the case of a lower bound restraint (usually, the material has a zero concentration in the zone under consideration).

The optimality condition is, then,

$$
\operatorname{EVREF}_{\mathrm{i}}(\mathrm{z}) \quad\left\{\begin{array}{l}
<\text { constant, for } \psi_{\mathrm{i}}(\mathrm{z})={ }^{\min } \psi_{\mathrm{i}}(\mathrm{z}) \\
=\text { constant, for }{ }^{\min } \psi_{\mathrm{i}}(\mathrm{z}) \leq \psi_{\mathrm{i}}(\mathrm{z}) \leq{ }^{\max } \psi_{\mathrm{i}}(\mathrm{z}) \\
>\text { constant, for } \quad \psi_{\mathrm{i}}(\mathrm{z})={ }^{\max } \psi_{\mathrm{i}}(\mathrm{z})
\end{array}\right.
$$

More details concerning the derivation of the above expressions can be found in Ref. 17.

\subsection{DISCRETE MESH IMPLEMENTATION}

The angular flux and adjoint distributions are expanded in spherical harmonics $\mathrm{Y}(\underline{\Omega})$ :

$$
\begin{aligned}
& \phi(\underline{\mathrm{r}}, \mathrm{E}, \underline{\Omega})=\sum_{\ell=0}^{\mathrm{L}} \sum_{\mathrm{m}=-\ell}^{\ell} \phi_{\ell m}(\underline{\mathrm{r}}, \mathrm{E}) \mathrm{Y}_{\ell \mathrm{m}}(\underline{\Omega}) \\
& \phi^{+}(\underline{\mathrm{r}}, \mathrm{E}, \underline{\Omega})=\sum_{\mathrm{k}=0}^{\mathrm{K}} \sum_{\mathrm{n}=-\mathrm{k}}^{\mathrm{k}} \phi_{\mathrm{kn}}^{+}(\underline{\mathrm{r}}, \mathrm{E}) \mathrm{Y}_{\mathrm{kn}}(\underline{\Omega})
\end{aligned}
$$

and using the ortho-normality property:

$$
\int_{4 \pi} \mathrm{d} \underline{\underline{\Omega}} \mathrm{Y}_{\ell \mathrm{m}}(\underline{\Omega}) \mathrm{Y}_{\ell^{\prime} \mathrm{m}^{\prime}}^{*}(\underline{\Omega})=\delta_{\ell \ell^{\prime}} \delta_{\mathrm{mm}}
$$

and the relation: 


$$
\mathrm{Y}_{\ell \mathrm{m}}(\underline{\Omega})=(-1)^{\mathrm{m}} \mathrm{Y}_{\ell,-\mathrm{m}}^{*}(\underline{\Omega})
$$

the multigroup expression for $\mathrm{AC}$ can be written as follows:

$$
\mathrm{AC}(\underline{\mathrm{r}})=\sum_{\mathrm{g}=1}^{\mathrm{G}} \Sigma_{\mathrm{t}}^{\mathrm{g}} \sum_{\ell=0}^{\mathrm{L}} \sum_{\mathrm{m}=-\ell}^{\ell}(-1)^{\mathrm{m}} \phi_{\ell \mathrm{m}}^{\mathrm{g}}(\underline{\mathrm{r}}) \phi_{\ell-\mathrm{m}}^{+\mathrm{g}}(\underline{\mathrm{r}})
$$

where the flux moments are defined by:

$$
\phi_{\ell \mathrm{m}}^{\mathrm{g}}(\underline{\mathrm{r}}) \equiv \int_{4 \pi} \mathrm{d} \underline{\Omega} \phi^{\mathrm{g}}(\underline{\mathrm{r}}, \underline{\Omega}) \mathrm{Y}_{\ell \mathrm{m}}^{*}(\underline{\Omega}) .
$$

The multigroup expression used for the calculation of the $\mathrm{BC}$ term is:

$$
\mathrm{BC}(\underline{\mathrm{r}})=\sum_{\ell=0}^{\mathrm{L}} \frac{4 \pi}{(2 \ell+1))} \sum_{\mathrm{m}=-\ell}^{\ell}(-1)^{m} \sum_{\mathrm{g}=1}^{G} \sum_{\mathrm{h}=1}^{G} \phi_{\ell \mathrm{m}}^{\mathrm{g}}(\underline{\mathrm{r}}) \sum_{\mathrm{s} \ell}^{\mathrm{g} \rightarrow \mathrm{h}} \phi_{\ell-\mathrm{m}}^{+\mathrm{h}}(\underline{\mathrm{r}})
$$

In deriving this equation the differential scattering cross section has been expanded into a Legendre polynomial series:

$$
\sigma_{\mathrm{s}}^{\mathrm{g} \rightarrow \mathrm{h}}(\mu)=\sum_{\ell=0}^{\mathrm{L}} \sigma_{\mathrm{s} \ell}^{\mathrm{g} \rightarrow \mathrm{h}} \mathrm{P}_{\ell}(\mu)
$$

where

$$
\sigma_{\mathrm{s} \ell}^{\mathrm{g} \rightarrow \mathrm{h}}=\frac{2 \ell+1}{2} \int_{-1}^{1} \sigma_{\mathrm{s}}^{\mathrm{g} \rightarrow \mathrm{h}}(\mu) \mathrm{P}_{\ell}(\mu) \mathrm{d} \mu
$$

and

$$
\int_{-1}^{1} \mathrm{P}_{\ell}(\mu) \quad \mathrm{P}_{\ell^{\prime}}(\mu) d \mu=\frac{2}{2 \ell+1} \delta_{\ell \ell^{\prime}}
$$

Use is also made of the spherical harmonics addition theorem:

$$
\mathrm{P}_{\ell}(\mu)=\frac{4 \pi}{2 \ell+1} \sum_{\mathrm{m}=-\ell}^{\ell} \mathrm{Y}_{\ell \mathrm{m}}^{*}\left(\underline{\Omega}^{\prime}\right) \quad \mathrm{Y}_{\ell \mathrm{m}}(\underline{\Omega})
$$

where $\mu$ is $\underline{\Omega} \bullet \underline{\Omega}^{\prime}$, the cosine of the polar angle between the two directions $\underline{\Omega}$ and $\underline{\Omega}^{\prime}$. The multigroup expression used for the calculation of the $\mathrm{FC}$ term is:

$$
\mathrm{FC}(\underline{\mathrm{r}})=\sum_{\mathrm{h}=1}^{\mathrm{G}} \chi^{\mathrm{h}} \phi^{+\mathrm{h}}(\underline{\mathrm{r}}) \sum_{\mathrm{g}=1}^{\mathrm{G}} v \Sigma_{f}^{\mathrm{g}} \phi^{\mathrm{g}}(\underline{\mathrm{r}})
$$


The expression for the normalization integral, NI, is like the sum of Eq. (23) over all the materials which contribute to fission.

The moments of the flux and of the adjoint distributions $\left[\phi_{\ell \mathrm{m}}^{\mathrm{g}}(\underline{\mathrm{r}})\right.$ and $\left.\phi_{\ell \mathrm{m}}^{+\mathrm{g}}(\underline{\mathrm{r}})\right]$ are calculated routinely by DORT and are transferred from DORT to SWIF via the XTROUT file. The DORT adjoint moments are inverted in SWIF before being used for the calculation of the effectiveness functions. The inversion, both in the energy and in the angular variables, is done as follows:

$\phi_{\ell \mathrm{m}}^{+\mathrm{g}}(\underline{\mathrm{r}})=(-1)^{\ell+\mathrm{m}}\left[\phi_{\ell \mathrm{m}}^{+(\mathrm{G}-\mathrm{g}+1)}\right]_{D O R T}$.

This inversion produces the physically meaningful adjoint.

The cross sections $\sigma_{\mathrm{t}}^{\mathrm{g}}$ and $\sigma_{\mathrm{s} \ell}^{\mathrm{g} \rightarrow \mathrm{h}}$ are the standard DORT cross sections.

\subsection{VARIATION OF COMPOSITION}

The variation in the volume fraction of the $i^{\text {th }}$ material in the $\mathrm{n}^{\text {th }}$ iteration is calculated from the expression:

$\delta \psi_{\mathrm{i}}^{\mathrm{n}}(\mathrm{z}) \equiv \psi_{\mathrm{i}}^{\mathrm{n}}(\mathrm{z})-\psi_{\mathrm{i}}^{\mathrm{n}-1}(\mathrm{z})=\mathrm{A}_{\mathrm{i}}^{\mathrm{n}} \mathrm{Q}_{\mathrm{e}, \mathrm{i}}^{\mathrm{n}-1}(\mathrm{z})+\mathrm{B}_{\mathrm{i}}^{\mathrm{n}} \mathrm{Q}_{\mathrm{c}, \mathrm{i}}^{\mathrm{n}-1}(\mathrm{z})$

where $Q_{e, i}$ is the EVREF of material i pertaining to the performance parameter to be maximized or minimized, and $Q_{c, i}$ in the EVREF of material $i$ pertaining to the constraint. $A_{i}$ and $B_{i}$ are coefficients that determine the amplitude of the volume fraction variation per iteration.

There are four major steps in the determination of the new density distribution: Determination of the $A_{i}$ coefficients, determination of the $B_{i}$ coefficients, the calculation of the new density distributions [Eq. (25)], and readjustment of the new density distributions if they have overflowed the specified density limits.

With $\delta \psi_{i}^{n}(\mathrm{z})$ of Eq. (25), the variation of the constraint becomes:

$$
\delta \mathrm{F}_{\mathrm{c}}^{\mathrm{n}}=\sum_{\mathrm{i}=1}^{\mathrm{I}-1} \int \mathrm{d} \underline{\mathrm{r}} \mathrm{Q}_{\mathrm{c}, \mathrm{i}}^{\mathrm{n}-1}(\mathrm{z}) \delta \psi_{\mathrm{i}}(\mathrm{z})=\sum_{\mathrm{i}=1}^{\mathrm{I}-1} \int \underline{\mathrm{d}} \underline{\mathrm{r}}\left[\mathrm{A}_{\mathrm{i}}^{\mathrm{n}} \mathrm{Q}_{\mathrm{c}, \mathrm{i}}^{\mathrm{n}-1}(\mathrm{z}) \mathrm{Q}_{\mathrm{e}, \mathrm{i}}^{\mathrm{n}-1}(\mathrm{z})+\mathrm{B}_{\mathrm{i}}^{\mathrm{n}} \mathrm{Q}_{\mathrm{c}, \mathrm{i}}^{\mathrm{n}-1}(\mathrm{z}) \mathrm{Q}_{\mathrm{c}, \mathrm{i}}^{\mathrm{n}-1}(\mathrm{z})\right]
$$

The coefficients $\mathrm{B}_{\mathrm{i}}^{\mathrm{n}}$ are determined to give $\delta \mathrm{F}_{\mathrm{c}, \mathrm{i}}^{\mathrm{n}}=0$, that is:

$$
\mathrm{B}_{\mathrm{i}}^{\mathrm{n}}=-\mathrm{A}_{\mathrm{i}}^{\mathrm{n}}\left[\int \mathrm{dr} \mathrm{Q}_{\mathrm{c}, \mathrm{i}}^{\mathrm{n}-1}(\mathrm{z}) \quad \mathrm{Q}_{\mathrm{e}, \mathrm{i}}^{\mathrm{n}-1}(\mathrm{z})\right] /\left[\int \mathrm{d} \underline{\mathrm{r}} \mathrm{Q}_{\mathrm{c}, \mathrm{i}}^{\mathrm{n}-1}(\mathrm{z})^{2}\right], \quad \mathrm{i}=1,2, \ldots, \mathrm{I}-1 .
$$

Inserting $\mathrm{B}_{\mathrm{i}}^{\mathrm{n}}$ [Eq.(27)] back into Eq.(26) one gets: 


$$
\delta \psi_{\mathrm{i}}^{\mathrm{n}}(\mathrm{z})=\mathrm{A}_{\mathrm{i}}^{\mathrm{n}}\left[\mathrm{Q}_{\mathrm{e}, \mathrm{i}}^{\mathrm{n}-1}(\mathrm{z})-\frac{\int \mathrm{d} \underline{\mathrm{r}} \mathrm{Q}_{\mathrm{c}, \mathrm{i}}^{\mathrm{n}-1}(\underline{\mathrm{r}}) \mathrm{Q}_{\mathrm{e}, \mathrm{i}}^{\mathrm{n}-1}(\mathrm{z})}{\int \mathrm{d} \underline{\mathrm{r}}\left[\mathrm{Q}_{\mathrm{c}, \mathrm{i}}^{\mathrm{n}-1}(\mathrm{z})\right]^{2}} \mathrm{Q}_{\mathrm{c}, \mathrm{i}}^{\mathrm{n}-1}(\mathrm{z})\right] .
$$

The $A_{i}^{n}$ coefficients are calculated by SWIF using Eq.(28) as follows:

$$
\mathrm{A}_{\mathrm{i}}^{\mathrm{n}}=\mathrm{a}_{\mathrm{i}}^{\mathrm{n}} /\left[\mathrm{Q}_{\mathrm{e}, \mathrm{i}}^{\mathrm{n}-1}\left(\mathrm{z}_{\mathrm{m}}\right)-\frac{\int \mathrm{d} \underline{\underline{r}} \mathrm{Q}_{\mathrm{c}, \mathrm{i}}^{\mathrm{n}-1}(\mathrm{z}) \mathrm{Q}_{\mathrm{e}, \mathrm{i}}^{\mathrm{n}-1}(\mathrm{z})}{\int \mathrm{d} \underline{\mathrm{r}}\left[\mathrm{Q}_{\mathrm{c}, \mathrm{i}}^{\mathrm{n}-1}(\mathrm{z})\right]^{2}} \mathrm{Q}_{\mathrm{c}, \mathrm{i}}^{\mathrm{n}-1}\left(\mathrm{z}_{\mathrm{m}}\right)\right] \text {, }
$$

where $z_{m}$ denotes the zone in which the volume fraction change will be the maximum (in absolute value) and $a_{i}^{n}$ are the input data specified in the 12* array of SWIF. The value of $a_{j}^{n}$ should be the maximum volume fraction change desired for material $i$ at the $\mathrm{n}^{\text {th }}$ iteration (in the units used for the material densities).

Details about the derivation of the above expressions can be found in Ref. 2.

\subsection{CONVERGENCE CONDITION}

The criterion used for testing how close is the optimization process (which is iterative) to convergence is a direct implementation of the optimality condition defined in Eq. (11). One possible implementation is the following: Over the zones in which material $i$ of variable concentration did not reach its lower or upper concentration limits,

$$
\left|\mathrm{Q}_{\mathrm{i}}^{\mathrm{n}}(\mathrm{z}) / \overline{\mathrm{Q}}^{\mathrm{n}}-1\right|_{\max } \leq \varepsilon, \quad \text { for all zones and for } \mathrm{i}=1,2, \ldots \mathrm{I}-1
$$

where $\overline{\mathrm{Q}}^{\mathrm{n}}$ is the average value of the EVREF in iteration $\mathrm{n}$; the averaging is over all the zones which participate in the optimization and over all the I-1 materials, excluding those combinations of zones and materials for which the volume fraction reached its lower or upper permissible limit. The subscript "max" implies the maximum relative deviation from the average. This deviation should not exceed the value $\varepsilon$-- a user specified parameter.

In addition to the condition of Eq. (30), there is a need to check the situation in all the zones in which any of the I-1 materials reached its concentration limit.

Additional information on the original SWAN methodology can be found in Refs. 1, 2 and 17. 


\section{THE OPTIMIZATION PROCESS}

Before starting the optimization process, a reference system geometry and composition is defined. The user needs also to define the number and location of zones in the system. A zone in SWAN is defined as a region the composition of which is kept uniform throughout the optimization process. A zone can be as small as a single interval, and as big as the entire system.

In addition, the user has to decide if he/she wants to consider the effect of candidate constituents and, if so, must add them to the system constituents. A candidate constituent is defined as a potential constituent that is not included in the reference system, but may be included in the optimal system. Alternatively, the user may be interested in getting the EVREF or ECREF of certain materials. All of these materials have to be included in the system. If the volume fraction of a given material the user wants to get its EVREF is zero, the user should assign to this material an infinitesimally small concentration (e.g., $10^{-15}$ ).

The optimization process is iterative. Each iteration starts with the calculation of the flux distribution followed by the calculation of one or, in case of performance parameter of the form of a ratio of functionals, two adjoint function distributions. Using these distributions and the crosssections, SWAN then calculates the EVREF of all the constituents defined by the user to have variable concentration in each of the specified zones. The EVREF are then used to guide an automated redistribution of the system constituents that will improve the performance parameter of interest. This iterative process continues until either an optimality condition or the maximum number of iterations specified by the user is reached. Illustrations of the optimization process are given in Section 7. 


\section{2D-SWAN STRUCTURE}

\subsection{OVERALL STRUCTURE}

The structure of the 2-D SWAN code is similar to that of the 1-D SWAN ${ }^{2}$. The primary difference is that the 1-D transport code $\mathrm{ANISN}^{40}$ was replaced by its 2-D counterpart DORT. ${ }^{41}$ Another difference is that the cross section library format was changed to match the requirement of DORT.

The 2-D SWAN code consists of three primary modules: GIP ${ }^{42}$, DORT ${ }^{41}$ and SWIF $^{2,43}$. GIP converts an ANISN binary cross-section file (ordered by material) to another binary crosssection file that has the format required by DORT (ordered by group). DORT is a twodimensional discrete ordinates program that calculates the neutron and/or photon flux distribution, $\mathrm{k}_{\mathrm{eff}}$ and reaction rates. It can also solve the adjoint equation. The SWIF calculates the Effectiveness Functions and performs a search of the optimal system composition. The flow of data between the three modules and from/into input/output units is controlled by a new driver program called DORIF.

Figure 5.1 shows a general flowchart of the DORIF sequence. The general sequence of steps that is required for each iteration of the optimization process is as follows:

1. Define the reference system composition, geometry and zones.

2. Create the group-ordered cross-section files for both direct and adjoint cases.

3. Using these cross-section libraries and the current material density distribution, DORT calculates the flux, reaction rates and adjoint functions across the system. For criticality problems $\mathrm{k}_{\mathrm{eff}}$ is also calculated.

4. Using the resulting fluxes, adjoint functions, the current material density distribution and the original ANISN material-ordered cross section file, SWIF calculates the user's specified effectiveness functions. If only those functions are required, then this is the end of the sequence. If, on the other side, the user requests an optimization, the SWIF continues to the next step.

5. Using the EVREFs, SWIF calculates a zone-wise modified system composition. If more iterations are required then steps 3 to 5 are repeated.

This sequence of iterations is terminated when the maximum number of iterations, specified by the user, has been reached. Automatic convergence test algorithms will be added to DORIF in the future.

Following is a list of the main programs DORIF is composed of, along with a brief description of their function:

SWAN2D - A master program that fixes the magnitude of the common array.

DRIV - Opens the required files, reads the driver data, controls the flow of calculations, updates the flux guess for the next iteration, checks if the maximum number of 
iterations is reached and writes the new volume fractions of the materials for use in the next iteration, if required. 


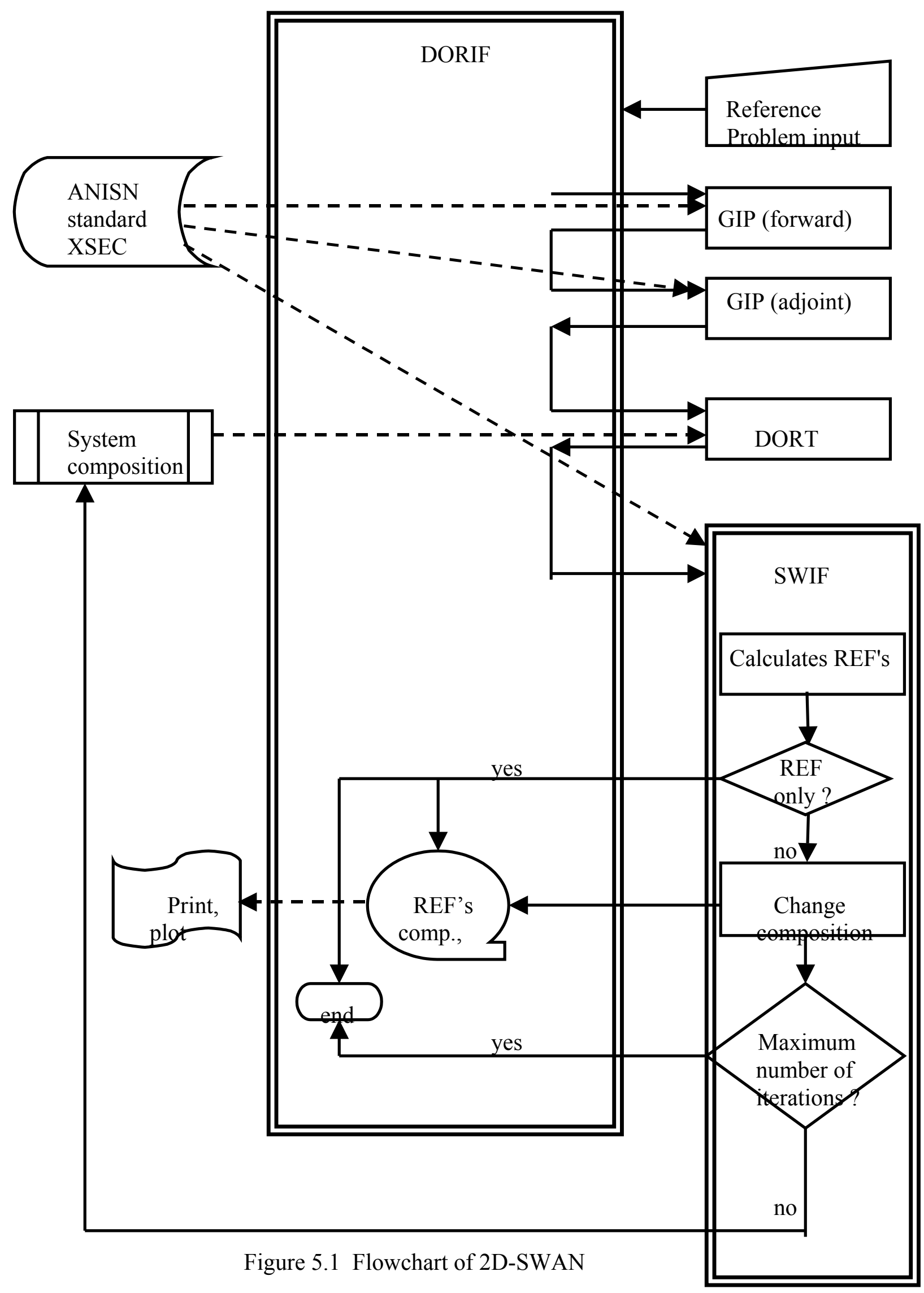


SHUFFLE - Reads the user's input data and splits it to make the input for GIP, DORT and SWIF.

FIDO - $\quad$ A short routine that addresses the reading of the user's input to AREAD.

AREAD - $\quad$ Reads explicitly the user's input, translates it and splits into different sub-input files as required (see later).

AWRITE - Writes output data.

CWRITE - Writes output data.

APUNCHOL-Writes output data in FIDO format.

GIP - $\quad$ Prepares the material-ordered cross-section file from an ordinary ANISN (groupordered) cross-section file. For more details see Reference 42.

DORT - Calculates the scalar flux, flux angular moments, activities and, for eigenvalue problems, also $\mathrm{k}_{\text {eff. }}$. Also calculates adjoint scalar flux and its angular moments. For more details see Reference 41.

SWIF - Calculates Effectiveness Functions and modifies the volume fraction of the materials of variable concentration in the search for the optimal system composition. For more details see Reference 43.

\subsection{THE SWIF OPTIMIZATION MODULE}

A flowchart of the SWIF optimization program is included in Figure 5.1. SWIF uses the same material-ordered cross section file used by GIP. The rest of the input data for SWIF is transferred from DORT. There are three or four input files: The first file includes a general description of the geometry needed by SWIF. DORT was modified in order to create such a file. The second file is the standard flux output file created by DORT; it is in the "VARFLM" format. The third file is similar to the second but containing adjoint flux data. The fourth file is needed only if the problem requires solution of two adjoint equations. If used this fourth file is to contain the second adjoint data.

Following is a list of the modules SWIF is composed of along with a brief description of their function:

SWIF - $\quad$ A routine that links between SWAN2D and the functional modules.

SETUP - $\quad$ Reads general data from the relevant file created by DORT and builds the addresses of the various arrays. It also controls the reading of the rest of the input data, controls the effectiveness function calculations and, if necessary, controls the creation of a new composition profile. 
UNIT3 - $\quad$ Reads the geometry data required by SWIF. Also reads the forward and adjoint fluxes from the standard VARFLM files created by DORT.

RDBLIB - Reads from the ANISN binary file the relevant cross-section data needed for SWIF.

EFFECT - Calculates the zone-wise EVREF for the materials of variable concentration.

NEWDEN - Calculates the new composition profile and updates the specified output files.

\subsection{GIP AND DORT MODIFICATIONS}

\subsubsection{GIP}

The original GIP reads the cross-section file that is ordered by materials (in ANISN format) from logical unit 9 and writes this data ordered by group on logical unit 8 . Since two GIP runs are required, one for the forward run and the second for the adjoint runs, the numbering of the logical units is set by DORIF. While the logical unit number for the ANISN ordinary file is set as before to 9 , The output cross-section file is now assigned to logical unit 7 for the forward run and to 8 for the adjoint runs.

The input for GIP is read from a logical unit 40 rather than from the standard logical unit 5. This was done in order to separate the input for GIP from those for DORT and SWIF. Since GIP is not run as a stand-alone module, some modifications were done in it in order to enable the user to use the ordinary ANISN X-SEC library each time GIP is called.

\subsubsection{DORT}

DORT uses the standard logical units 5 and 6 as, respectively, the input and output files. Some modifications were made in the DORT routines that treat the reading of the input, so that the user can read it from a several of input logical units. This enables the user to avoid repeating almost the same input for the forward and the adjoint (or the two adjoint) cases. This also enables the user to set the material density data (the $12 *$ array in DORT) on a separate file so that only this file can be replaced by SWIF when a new composition profile is generated.

One of the advantages of the multi input units set for DORIF is that it enables DORT to use the fluxes with its moments from a previous material iteration as an initial flux guess for the next material iteration. This option was found capable of considerably reducing the time for DORT convergence.

The original DORT is set to read the input data from logical unit 41. The FFREAD and FIDAS routines of DORT were modified to enable reading the data from other logical units. The new options added to these routines are the following: 
$\mathrm{nx}$ - Tells the input reading routine to start reading data from logical unit $\mathrm{n}$ just from the location where it was after the last time of data reading, i.e. the input file should not be rewinded.

ny - Tells the input reading routine to start reading data from the beginning of logical unit $\mathrm{n}$; i.e. first rewind this input file and then read from it.

Another option added to this routine is introduction of "Pv" anywhere in an input array; when introduced it multiplies all the elements of the input array, up to the position of "P" in this array, by the real number "v".

The SHUFFL routine reads the entire input, as prepared by the user and splits it between the various logical units, as defined in advance in DORIF or by the user. When this routine finds the following card

() $+\mathrm{n}$

with a blank in the $3^{\text {rd }}$ column, it transfers the data that follows to logical unit $\mathrm{n}$ until either the end of input is reached or this special card is read again.

Some modifications in material identification by DORT were needed in order to enable interfacing between DORT and SWIF. The original definitions for arrays $10 \$$ and $11 \$$ (MIXT and NUCL arrays) of DORT were related to the column number of the proper materials (or mixtures) in the cross section matrix, as produced by GIP. In DORIF, they were changed to the material number, as read from the ANISN binary cross-section file by GIP, or to any free number for mixtures. The assignment of the material numbers to the column numbers is done via the $13 \$$ array (MATL) of the DORT input. As a result some modifications were done in the routine MACMX that prepares the cross-sections for mixtures.

An input restriction is that ISCTM must be always positive. This is because SWIF, at this stage, cannot handle variable order of scattering.

\subsection{I/O FILES}

\subsubsection{GIP}

fort. 1 - Local file

fort. 2 - Local file

fort. 3 - Local file

fort.4 - Local file

fort.7 - Binary-output file of cross-sections for forward calculations of DORT.

fort.8 - Binary-output file of cross-sections for adjoint calculations of DORT.

fort.9 - Binary-input file of cross-sections in ANISN format.

fort.40 - Input file for GIP.

\subsubsection{DORT}

fort.1 - Local file 
fort.3 - Local file

fort. 4 - Local file

fort.7 - Binary-output file of cross-sections for forward calculations as done in GIP.

fort.8 - Binary-output file of cross-sections for adjoint calculations as done in GIP.

fort.21 - ASCII file containing the material composition profile (12** array) for DORT.

fort.25 - ASCII file (optional) contains the common input of the two (or three) cases.

fort.35 - ASCII file containing the flux guess input (93* and $95^{*}$ arrays) for the first iterations. In the next iterations it becomes dummy.

fort.41 - Master input file for DORT.

fort.61 - Binary file containing fluxes and moments for forward case in VARFLM format. It is an output file for each odd iteration and input file for each even iteration.

fort.62 - As fort.61, but it is an input file for each odd iteration and output file for each even iteration.

fort.63 - As fort.61 but for the first adjoint case.

fort.64 - As fort.63, but it is an input file for each odd iteration and output file for each even iteration.

fort.65 - As fort.61 but for the second adjoint case, if occurred.

fort.66 - As fort.65, but it is an input file for each odd iteration and output file for each even iteration, if occurred.

fort.67 - ASCII file that updated the flux guess input unit (NTFLX) and the flux output unit (NTFOG) in the $61 \$$ array of DORT. Enables using of fluxes and moments from previous iteration.

fort.71 - Binary file containing the distributed source (NTDSI in the $61 \$$ array of DORT). It is required when INPSRM $>0$.

fort. 82 - Local file

fort.83 - Local file

fort.84 - Local file

fort.85 - ASCII file (optional) containing the direct source distribution for the forward case (the $96^{*}, 97 *, 98^{*}$ arrays of the input to DORT).

fort.91 - Local file

fort.92 - Local file

fort.93 - Local file

fort.94 - Local file

fort.95 - Local file

fort.201 - Binary file containing necessary data, such as geometry, activities, $\mathrm{k}_{\mathrm{eff}}$, to be transmitted from DORT to SWIF

fort.202 - ASCII file with the edit-out of fort.201

\subsubsection{SWIF}

fort.9 - Binary-input file of cross-sections in ANISN format.

fort.10 - ASCII file that accumulates all the profiles from fort.21 of DORT and fort.22 of SWIF with the iterations.

fort.22 - ASCII file containing the material composition profile (11** array) for SWIF.

fort.30 - Binary file that dumps most of the input and output of SWIF if the trigger SAVE in the $1 \$$ array of SWIF is set to 1 . 
fort.34 - ASCII file that contains general information, such as zone structure and, for every iteration: the iteration number, the volume fractions of materials for which the EVREF were calculated, EVREF values, as well as the system $k_{\text {eff }}$ and the mass of the materials. (Like in SWANS ${ }^{43}$, see later appendix about the structure of this file.)

fort.42 - ASCII file which contains the input for SWIF.

fort.50 - Local file

fort.61 - Binary fluxes and moments files as edited by DORT.

fort.62 - Binary fluxes and moments files as edited by DORT.

fort.63 - Binary fluxes and moments files as edited by DORT.

fort.64 - Binary fluxes and moments files as edited by DORT.

fort.65 - Binary fluxes and moments files as edited by DORT.

fort.66 - Binary fluxes and moments files as edited by DORT.

fort.201- Binary file containing necessary data, such as geometry, activities, $k_{\text {eff }}$, to be transmitted from DORT. 


\section{INPUT DATA DESCRIPTION}

A general structure of the input to DORIF (2D-SWAN) is given in Figure 6.1. This input data is for problems that require a solution of two adjoint equations (here denoted as "adjoint- $\mathrm{H}$ " and

\begin{tabular}{|c|}
\hline DORIF DRIVER (2 lines) \\
\hline $\begin{array}{l}=\text { dort } \\
\text { DORT INPUT SPECIFIC TO FORWARD CASE } \\
t\end{array}$ \\
\hline $\begin{array}{l}=\text { dort } \\
\text { DORT INPUT SPECIFIC TO ADJOINT-H CASE } \\
\mathrm{t}\end{array}$ \\
\hline $\begin{array}{l}=\text { dort } \\
\text { DORT INPUT SPECIFIC TO ADJOINT-L CASE } \\
\mathrm{t} \\
=\text { end }\end{array}$ \\
\hline $\begin{array}{l}()+25 \\
\text { DORT INPUT COMMON FOR ALL CASES }\end{array}$ \\
\hline $\begin{array}{l}()+40 \\
=\text { gip } \\
\text { GIP INPUT FOR FORWARD CASE } \\
=\text { end } \\
=\text { gip } \\
\text { GIP INPUT FOR ADJOINT CASE } \\
=\text { end }\end{array}$ \\
\hline $\begin{array}{l}()+35 \\
\text { DORT FLUX GUESS INPUT FOR THE THREE CASES }\end{array}$ \\
\hline $\begin{array}{l}()+67 \\
\text { DORT FLUX INPUT AND OUTPUT UNITS }\end{array}$ \\
\hline $\begin{array}{l}()+42 \\
\text { SWIF INPUT }\end{array}$ \\
\hline $\begin{array}{l}()+22 \\
\text { SWIF PROFILE INPUT ( } 11 * \text { ARRAY) }\end{array}$ \\
\hline $\begin{array}{l}()+21 \\
\text { DORT PROFILE INPUT (12* ARRAY) }\end{array}$ \\
\hline
\end{tabular}

Figure 6.1 A general layout of DORIF input 
"adjoint-L" or "Adjoint case") in addition to a solution of a flux equation ("Forward case"). Overall there are 11 input data blocks. The lines above/below each block title are input lines that designate block initiation/termination. Following is a detailed data input specifications for each of the input "blocks" of Fig. 6.1.

\subsection{DORIF DRIVER}

This input block includes only two cards that control the flow of GIP, DORT and SWIF:

CARD 6.1.1 - Title (12A4); A general description (Hollerith)

CARD 6.1.2 - Option specifications (6I3)

1. IFIL - Preparation of input data files

0 - Use existing data files

1 - Prepare new data files (recommended)

2. IOPT - Sequence of calculations

0 - DORT run only

1 - SWIF run only

2 - Start with DORT followed by SWIF (recommended)

3 - Start with SWIF followed by DORT

3. IEF - Purpose of SWIF calculations

0 - Optimization Calculations

1 - Effectiveness Functions only

4. IMFL - Modifications to DORT and SWIF profile input files

$-1-$ SWIF will initialize contents of files (recommended for IEF $=1$ )

0 - SWIF will not modify contents of files

$1-$ SWIF will modify contents of files (recommended for IEF $=0$ )

5. IMAX - Maximum number of iterations on material density distributions $\{$ for IOPT $\geq 2$ and $\mathrm{IEF}=0$ \}

1 - for Effectiveness calculations (IEF $=1)$

6. ISUPR - Number of Iteration from which the SWIF output is suppressed (2 is recommended).

\subsection{DORT INPUT SPECIFIC FOR "X" CASE}

The next three input blocks contain the specific input for each of the three DORT runs: one forward run and two adjoint runs: adjoint-H and adjoint-L. The last of the blocks should be terminated with an "=end" line (as shown in Fig. 6.1). For detailed information about the meaning of the different input parameters see the manual of DORT ${ }^{41}$.

CARD 6.2.1 - = =dort (as shown in Fig. 6.1).

CARD 6.2.2 - Title card - begins with " in the first column.

CARD 6.2.3 - 61\$\$ - Data set logical unit reference numbers:

1. NTFLX - Flux input unit - Set to 0 at the beginning.

2. NTFOG - Flux output unit - At the beginning set to:

61 - for forward case 
62 - for adjoint-H (first adjoint) case

63 - for adjoint-L (second adjoint) case

In the second iteration, for the three cases, DORT sets automatically NTFLX to 61,62 and 63 and NTFOG to 64, 65 and 66. In the third iteration DORT sets NTFLX back to 64,65 and 66 and NTFOG back to 61,62 and 63 and so on.

3. NTSIG - Cross-section unit, as created by GIP. Should be always:

7 for forward case

8 for adjoint- $\mathrm{H}$ and adjoint- $\mathrm{L}$ cases.

4. NTBSI - 0

5. NTDSI - Distributed source input unit scratch - set to 71 for each case.

Terminate this input array with e. e means skip to the end of this array.

CARD 6.2.4 - $\quad 67 \mathrm{y} \quad$ for the first (forward) case

$67 \mathrm{x}$ for the other (adjoint) cases

The input data is to be read from logical unit 67. For the first case the data is read from the beginning of this logical unit. For the other cases it is read from the end point reached in the previous reading from this logical unit (see section 6.6).

CARD 6.2.5 - $\quad 25 y$

Switches to the beginning of logical unit 25 .

CARD 6.2.6 - $\quad 62 \$ \$$ - Define the special features for each case"

- For the forward case, add the two cards:

a1 0

a30 N A35 2 e

- For each adjoint case add the two cards:

a1 1

a30 $\mathrm{Ne}$

The notation An means move to the $\mathrm{n}^{\text {th }}$ entry in the current array.

The first entry (IADJ) in $62 \$ \$$ array defines if the problem is forward (0) or adjoint (1) calculation.

The $30^{\text {th }}$ entry (INPSRM) in this array defines the distributed source input option:

- 0 The source is set to 0 or read from unit NTDSI if NTDSI $>0$.

- 1 SIJ(I,J) is read from $96^{* *}$ array for each group. See card 6.2.17.

- $2 \mathrm{SIJ}(\mathrm{I}, \mathrm{J}) * \mathrm{SG}(\mathrm{G})$, read from $96^{* *}$ and $98^{* *}$ arrays. See cards 6.2 .17 and 6.2.19.

- $3 \mathrm{SI}(\mathrm{I}) * \mathrm{SJ}(\mathrm{J}) * \mathrm{SG}(\mathrm{G})$, read from the $96^{* *}, 97^{* *}$ and $98^{* *}$ arrays. See cards 6.2.17, 6.2.18 and 6.2.19.

NTDSI is required ALWAYS if INPSRM $>0$.

The $35^{\text {th }}$ entry (IACT) in this array defines the number of region and pointwise activities to be calculated. It is defined only for the first (i.e., forward) 
case which is set to 2 . In the other (adjoint) cases it takes the default value, i.e. 0 .

CARD 6.2.7- $\quad 67 y$

Switch reading the input from the beginning of logical unit number 67.

CARD $6.2 .8-\quad 25 \mathrm{x}$

Switch reading the input from logical unit 25 starting from the end point reached in the previous reading from logical unit 25.

CARD 6.2.9 - $\quad 63^{* *}$ - Define the special features for each case:

For each case add the following card:

- a2 XNF

The $2^{\text {nd }}$ entry $(\mathrm{XNF})$ in this array defines the normalization of the source for each of the three cases. When set to 0 the normalization is ignored. 0 is RECOMMENDED ALWAYS for the adjoint cases.

CARD 6.2.10 - $25 x$

Switch reading the input from logical unit 25 starting from the end point reached in the previous reading from logical unit 25.

CARD 6.2.11- 25x

Switch reading the input from logical unit 25 starting from the end point reached in the previous reading from logical unit 25.

CARD 6.2.12 - 25\$\$ - Activity data part I. ICMAT(IAC) materials to be used in activity calculations (length $|\mathrm{IACT}|)$. To be specified for forward calculation only.

ICMAT(IAC) is the location of the activity material IAC in $13 \$ \$$ array (See card 6.3.23). If negative, do not multiply the cross-section times the flux by the material density.

CARD 6.2.13 - 26\$\$ - Activity data part II. Specify ICPOS(IAC) cross section table position for activity (length $|\mathrm{IACT}|$ ). To be specified for forward calculation only.

CARD 6.2.14 - 27** - Activity data part III. Specify ACMUL(IAC) activity multiplier (length $|\mathrm{IACT}|)$. To be specified for forward calculation only.

The above three data arrays need to be specified for the $\mathrm{H}$ and $\mathrm{L}$ activities.

CARD 6.2.15 - $\mathrm{t}$

Terminates this data block. See DORT manual ${ }^{41}$.

CARD 6.2.16 - $\quad 35 \mathrm{y} \quad$ for the first (forward) case.

$35 \mathrm{x}$ for the other (adjoint) cases

The input data is to be read from logical unit 35. For the first case the data is read from the beginning of this logical unit. For the other cases it is read from 
the end point reached in the previous reading from this logical unit (see Section 6.5).

CARD 6.2.17 - 96** - The distributed source input for each case; part I. Specify $\mathrm{SIJ}(\mathrm{I}, \mathrm{J}), \mathrm{S}(\mathrm{I}, \mathrm{J})$ or $\mathrm{S}(\mathrm{I})$ (length $=\mathrm{ISMJM}$, ISMJM or IM) as INPSRM=1,2 or 3 . See card 6.2.6.

CARD 6.2.18 - 97** - The distributed source input for each case; part II. Specify $\mathrm{SJ}(\mathrm{J})($ length $=\mathrm{JM})$ only if INPSRM $=3$. See card 6.2.6.

CARD 6.2.19 - 98** - The distributed source input for each case; part III. Specify $\mathrm{SG}(\mathrm{G})($ length $=\mathrm{IGM})$ only if INPSRM $>1$. See card 6.2.6.

Each of the above three arrays must terminate by $\mathrm{t}$, as shown in Fig. 6.1.

CARD 6.2.20 - =end

Terminates this section as shown in Fig. 6.1. Specify for the last case ONLY.

\subsection{DORT INPUT COMMON FOR ALL CASES}

This data block specifies the common input for the forward and adjoint cases for DORT. It is assumed that the user is familiar with DORT and its instructions.

CARD 6.3.1 - $\quad($ ) +25 (See Fig. 6.1)

This specifies that all the following data will be inserted on logical unit 25 .

Note that column 3 is a blank.

CARD 6.3.2 - $\quad 62 \$ \$-$ Control parameters input (Integers):

For positions not defined below the default option is used. For more details or changes see the DORT manual ${ }^{41}$.

1. IADJ - Dummy. Its definition is $0 / 1$ for forward/adjoint calculation. This data is replaced by the value specified in card 6.2.6.

2. ISCTM - Maximum order of scattering.

Must be positive and the same in all the system.

3. IZM - Number of material zones.

4. IM - $\quad$ Number of spatial intervals in $1^{\text {st }}$ dimension (I).

Must be positive and the same for each $\mathrm{J}$ in the $2^{\text {nd }}$ dimension.

5. JM - Number of spatial intervals in the $2^{\text {nd }}$ dimension $(\mathrm{J})$.

6. IGM - Number of energy groups.

7. IHT - Position of total cross-section in cross-section table.

8. IHS - Position of self-scatter cross-section in cross-section table.

9. IHM - Length of cross-section in cross-section table for each energy group.

10. MIXL - Cross-section mixing table length.

11. MMESH - 0

12. MTP - Number of cross section sets to be read from NTSIG (see card 6.2.3.)

13. MTM - Total number of cross section sets including mixtures. 
14. IDFAC - Density factor. The input options are:

0 - not used

1 - Enter 3* array DNIJ(I,J) (see card 6.3.16) for modifying cross-sections.

15. MM - Maximum number of direction to be used.

Negative implies that a variable quadrature is used.

16. INGEOM - Geometry option:

0 - X-Y (infinite slab)

1 - R-Z (finite cylinder)

2 - R (infinite cylinder)

For more options see the DORT manual ${ }^{41}$.

17. IBL $-\quad$ Left boundary condition:

0 - void

1 - reflected

2 - periodic

3 - cylindrical

4 - fixed boundary source

5 - albedo

18. IBR - Right boundary condition. Same options as for IBL.

19. IBB - Bottom boundary condition. Same options as for IBL.

20. IBT - Top boundary condition. Same options as for IBL.

21. ISRMX - Maximum number of source (outer) iterations (default $=1$ ).

22. IFXMJ - Maximum number of flux (inner) iterations per group (default $=20$ ).

23. IFXMF - Final maximum number of flux (inner) iterations per group (no effect if 0 ).

24. MODE - Flux extrapolation model $($ default $=4)$. The options are:

0 - linear with negative fluxes set to 0

1 - linear with no fixup of negative fluxes

2 - scalar weighted

3 - zero weighted

4 - theta weighted (recommended in DORIF, especially for adjoint cases).

5 - vector weighted

25. KTYPE - Calculation type.

0 (fixed source) is the only option currently DORIF is set to handle.

26. IACC - Acceleration method used for the discrete ordinates solution. The options are:

0 - single groupwise rebalance factor

1 - diffusion acceleration

2 - partial current rebalance (default)

27. KALF - Rebalance stabilization method option:

0 - standard method

1 - alternate method

28. IGTYPE - Solution method. Set always to 0 (discrete ordinates).

29. INPFXM -Initial flux input option:

0 - flux read from logical unit NTFLX

1 - FIJ(I,J) read as $93^{* *}$ array for each group. See card 6.5.2.

2 - FIJ(I,J)*FG(G), uses $93 * *$ and $95^{* *}$ arrays. See cards 6.5.2 and 6.5.4.

$3-\mathrm{FI}(\mathrm{I}) * \mathrm{FJ}(\mathrm{J}) * \mathrm{FG}(\mathrm{G})$, uses $93 * *, 94 * *$ and $95^{* *}$ arrays. See cards 6.5.2, 6.5.3 and 6.5.4. 
30. INPSRM -Distributed source input options - same options as for INPFXM.

Dummy. It is overridden by the value specified in card 6.2.6

35. IACT - Number of region and pointwise activities to be calculated. If negative calculate only region activities.

Dummy. It is overridden by the value specified in card 6.2.6.

36. IRED - Balance table output control. Set to IZM to print balance table for each of the IZM zones.

CARD 6.3.3 - $\quad 41 \mathrm{x}$

Switch reading the input from logical unit 41 starting from the end point reached in the previous reading from logical unit 41 .

CARD 6.3.4 - e

This switches the input to the end of the array $62 \$ \$$.

CARD 6.3.5 - $\quad 63^{* *}$ - Control parameters input (floating point):

For positions not defined here the default option is used. For more details see DORT manual ${ }^{41}$.

1. TMAX - Maximum minutes of CPU time to be used for this problem. Ignored when set to 0

2. XNF - Dummy. It is overridden by the value specified in card 6.2.8.

End by e that instructs skipping to the end of this array.

CARD 6.3.6- $\quad 41 \mathrm{x}$

Switch reading the input from logical unit 41 starting from the end point reached in the previous reading from logical unit 41 .

CARD 6.3.7 - e

This switches the input to the end of the array $63^{* *}$.

CARD 6.3.8 - $\quad \mathrm{t}$

Terminates input data block 1 for DORT.

CARD 6.3.9 - $\quad \mathrm{t}$

Skips input data block 2 for DORT.

Cards 6.3.10 to 6.3 .13 constitute input data block 3 for DORT ${ }^{41}$ :

CARD 6.3.10 - 81** - W(M,MSET); Directional weights.

CARD 6.3.11 - $82 * *$ - EMU(M,MSET); $\mu$, cosine of angle with X or R direction.

CARD 6.3.12 - 83** - ETA(M,MSET); $\eta$, cosine of angle with $\mathrm{Z}$ or $\Theta$ direction.

CARD 6.3.13 - $\mathrm{t}$

Terminates input data block 3 for DORT. 
Cards 6.3.14 to 6.3 .24 constitute input data block 4 for DORT ${ }^{41}$ :

CARD 6.3.14 $-\quad 1^{* *}-\mathrm{CHI}(\mathrm{IG})$; fission spectrum fractions, $\chi$, by group.

CARD 6.3.15 - $\quad 2 * *-Z I N(J) ; Z$ or $\Theta$ fine mesh boundaries.

CARD 6.3.16 - $3^{* *}-$ DNIJ(I,J); density factor, if IDFAC $>0$ (see card 6.3.2).

Default is 1.0 for all the array.

CARD 6.3.17 - $\quad 4 * *-$ RIN(I,ISET); X or R fine mesh boundaries.

CARD 6.3.18 - $8 \$ \$$ - IJZN $(I, J) ;$ material zone by fine space mesh.

CARD 6.3.19 - 9\$\$ - IZMT(IJZN); material number (location in $13 \$ \$$ array - see card 6.3.23) by material zone.

CARD 6.3.20 - $10 \$ \$$ - MIXT(MIX); mixture ID numbers in the mixing table (MIXL $>0$ ). These numbers should be consistent with those defined in $13 \$ \$$ array - see Card 6.3.23.

CARD 6.3.21 - $11 \$ \$$ - NUCL(MIX); component ID numbers in the mixing table (MIXL $>0$ ). These numbers should be consistent with those defined in $13 \$ \$$ array - see Card 6.3.23.

CARD 6.3.22 - 21y

Switch reading the input from the beginning of logical unit 21.

CARD 6.3.23 - 13\$\$ - MATL(MT); list of material ID numbers of nuclides in the same order as in GIP (see card 6.4.9) and used in card 6.3.21, followed by material ID number of mixtures (chosen freely) as used in Card 6.3.20.

CARD 6.3.24 - 41x

Switch reading the input from logical unit 41 starting from the end point reached in the previous reading from logical unit 41 .

\subsection{GIP}

This data block includes the input for preparing the cross-section files in group-organized library format as required by DORT. Since forward and adjoint jobs require different ordering of the groups, it has to be specified twice. For details see Reference 42.

CARD 6.4.1 - $\quad()+40 \quad$ (See Fig. 6.1)

All the following data will be inserted on logical unit 40 . Note that $3^{\text {rd }}$ space is blank. 
Use cards 6.4.2 to 6.4.6 to create one input set for the forward case and one input set for the adjoint cases (the two adjoint cases use the same GIP library).

CARD 6.4.2 - $\quad$ =gip $\quad$ (See Fig. 6.1)

CARD 6.4.3 - $\quad$ Title (72 character description); A general description (Hollerith)

CARD 6.4.4 - $\quad 1 * *-$ Input parameters:

1. IGM - Number of energy groups

2. IHT - Position of total cross-section

3. IHS - Position of in-scatter cross-section

4. IHM - Length of cross section table

5. MS - Mixing table length. It is set to 0 in DORIF.

6. MCR - Number of materials to be read from cards. It is set to 0 in DORIF.

7. MTP - Number of materials to be read from the ordinary cross-section library file in binary format (as used by $\mathrm{ANISN}^{40}$ )

Each of the ISCT components for a given isotope is counted as a separate material.

8. MTM - Number of all the materials, including those read by GIP (MCR + MTP).

9. ITH - $0-$ for a forward case.

1 - for an adjoint case.

10. ISCT - Maximum order of scattering expansion

11. IPRT - $\quad 0$ - Print cross sections for all materials

1 - Do not print (recommended)

2 - Print only for mixtures

12. IOUT- $\quad 0$ - Output all materials (recommended)

1 - No output

2 - Output only for mixtures

13. IDOT-Output format

$0-$ ANISN

$1-$ DOT-III

2 - DOT-IV (recommended)

14. NBUF - Number of Kilo-Bytes buffer space $($ default $=60)$

End this array by e and t separated by a blank.

CARD 6.4.5 - $\quad$ 13\$\$ - Material ID numbers to be read from the ordinary cross-section library file in binary format, as used by ANISN $^{40}$ (MTP entries) .

End section by t.

CARD 6.4.6 - = =nd (See Fig. 6.1).

This card ends the input for a case.

\subsection{DORT FLUX GUESS INPUT FOR THE THREE CASES}


This data block specifies the input for Input Data Block 6 for DORIF (Fig. 6.1). A data block need to be specified for each DORT run; one for the forward solution and one for each adjoint run. It is assumed that the user is familiar with the manual of DORT ${ }^{41}$.

CARD 6.5.1 $-\quad()+35 \quad$ (See Fig. 6.1)

All the following data will be inserted on logical unit 35 . Note that the third space is blank.

Start with the input for a forward run.

CARD 6.5.2 - $93^{* *}-\mathrm{FIJ}(\mathrm{I}, \mathrm{J}), \mathrm{FIJ}(\mathrm{I}, \mathrm{J})$ or FI(I) as INPFXM $=1,2$, or 3.

CARD 6.5.3 $-94^{* *}-\mathrm{FJ}(\mathrm{J})$ if INPFXM $=3$.

CARD 6.5.4 - 95** $-\mathrm{FG}(\mathrm{IG})$ if INPFXM $>1$.

CARD 6.5.5 - $\quad 41 \mathrm{x}$

Switch reading the input from logical unit 41 starting from the end point reached in the previous reading from logical unit 41 .

Repeat Cards 6.5.2 to 6.5.5 for each adjoint run.

DORIF replaces the content of this file with updated fluxes in each optimization iteration.

\subsection{DORT FLUX INPUT AND OUTPUT UNITS}

DORIF changes the input and output logical units for the flux from one iteration to the next. The unit numbers are interchanged so that they are the same in each even DORIF iteration and the same in each odd DORIF iteration. In the first iteration DORIF uses the user specified initial flux (adjoint) guess (See Sec. 6.5). For any other iteration, DORIF uses for the initial flux (adjoint) the flux (adjoint) distribution (including all the angular moments) calculated in the previous iteration. This procedure considerably reduces the CPU time.

CARD 6.6.1 - $\quad()+67 \quad$ (See Fig. 6.1).

All the following data will be inserted on logical unit 67 . Note that the third space is blank.

Start with input for a forward run.

CARD 6.6.2 - $41 \mathrm{x}$

Switch reading the input from logical unit 41 starting from the end point reached in the previous reading from logical unit 41. In the next iteration DORIF replaces this card by defining the proper input and output logical units (NTFLX and NTFOG) in the 61\$ $\$$ array - see card 6.2.3.

CARD 6.6.3 - $\quad 41 \mathrm{x}$ 
Switch reading the input from logical unit 41 starting from the end point reached in the previous reading from logical unit 41. In the next iteration DORIF replaces this card by a proper card with the current INPFXM value the $29^{\text {th }}$ entry in $62 \$ \$$ array.

Repeat cards 6.6.2 and 6.6.3 also for each adjoint case.

\subsection{SWIF INPUT}

This data block describes the input required for preparing the system composition for the next iteration. All the data given in this block remains unchanged during the DORIF iterations. For more details see Ref. 43.

CARD 6.7.1 - $\quad()+42 \quad$ (See Fig. 6.1).

All the following data will be inserted on logical unit 42 . Note that the third space is blank.

CARD 6.7.2 - Title card. Its format is $(10 \mathrm{~L} 1,10 \mathrm{I} 1,15 \mathrm{~A} 4)$

The first 10 entries are indicators that control the printout in SWIF - the indicator can have one of two values: $\mathrm{T}$ for print or $\mathrm{F}$ for not to print. Following is a definition of the different printout control indicators:

Column Meaning

1 Parameters for /BULKBU/ common block.

2 Fluxes and currents of forward solution.

3 Fluxes and currents of adjoint solution(s) as given by DORT.

$4 \quad$ Fluxes and currents of adjoint solution(s) inverted in SWIF.

5 Cross-section data used by SWIF.

6 Terms of the Effectiveness Functions.

7 Density dependent input arrays.

8-10 Not used.

The next 10 entries are indicators that control the output of the revised density dependent data arrays:

Column

11 Not used in DORIF.

12 for SWIF $11 * *$ array (see section 6.8).

13 for DORT $12 * *$ array (see section 6.9).

14-20 Not used in DORIF.

The following options are available for columns 12 and 13:

0 - No output.

1 - Print only.

7 - Print and modify contents of files 21 and 22 (recommended by DORIF).

The last data is a title with a maximum of 60 characters.

DATA SEGMENT 1 : Initial Data 


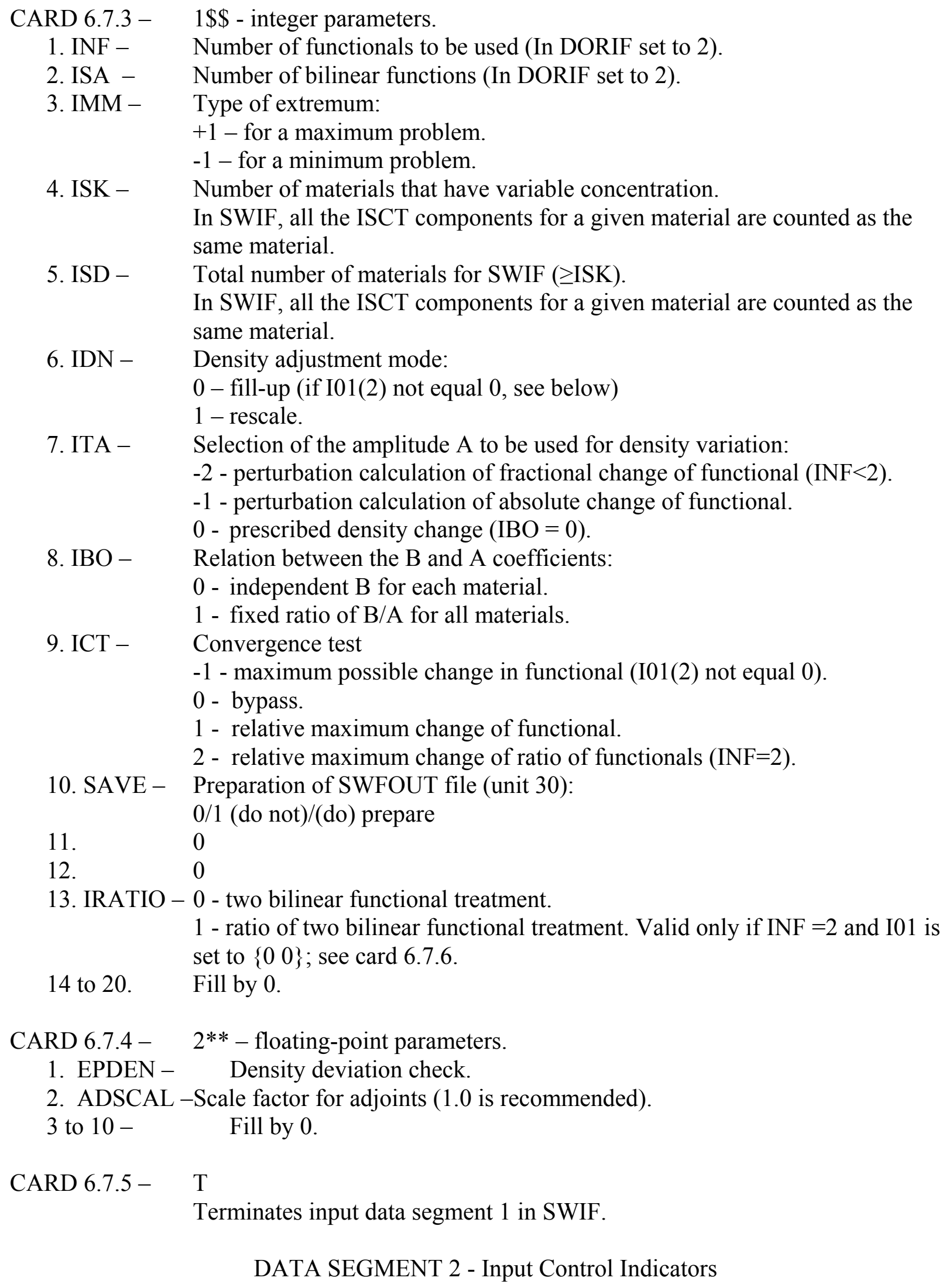


$\begin{array}{ll}\text { CARD 6.7.6 - } & 3 \$ \$-\text { I01 vector. } \\ & \text { Specify } 0 / 1 \text { for bilinear/weight functional (length INF). }\end{array}$

The weight functional option has not been tested so far in this version of DORIF.

CARD 6.7.7 - $\quad 4 \$ \$-$ ISAS $(\leq \mathrm{ISD})$

Number of materials that go into each adjoint source term (length ISA).

Fill with 0 is recommended.

CARD 6.7.8 - $\quad \mathrm{T}$

Terminates input data segment 2 in SWIF.

\section{DATA SEGMENT 3 - Material Specifications}

CARD 6.7.9 - $\quad 7 \$ \$$ - ID numbers of all materials (read from the ordinary cross-section library file in binary format, as used by ANISN library ${ }^{40}$ ) which have variable concentration (length $\left\{\mathrm{ISK}^{*}[\mathrm{ISCTM}+1]\right\}$ ).

CARD 6.7.10 - T

Terminates input data segment 3 in SWIF.

DATA SEGMENT 4 - Material Densities

CARD 6.7.11 - $\quad 8^{* *}$ - Maximum volume fraction of each material of variable concentration if it were to occupy all the volume available for all the materials of variable concentration (length IZM * ISK). For zones that have no materials of variable concentration set to 0 .

CARD 6.7.12 - $9 * *$ - Maximum permissible volume fraction of each material of variable concentration in each zone (length IZM * ISK).

For zones that have no materials of variable concentration set to 0 .

CARD 6.7.13 - $10^{* *}$ - Minimum permissible volume fraction of each material of variable concentration in each zone (length IZM * ISK).

For zones that have no materials of variable concentration set to 0 .

For any other zone, if any of the specified materials has an initial value of 0 , then this number should be set to $1 \mathrm{E}-15$.

CARD 6.7.14 - 22X

Switch reading the input from logical unit 22 starting from the end point reached in the previous reading from logical unit 22 (see section 6.8).

CARD 6.7.15 - 12** - Coefficients (A) for density modification (length [ISK-1]). 
Use when $\mathrm{IEF}=0$.

CARD 6.7.16 - 51** - Convergence parameters for substitution effectiveness functions (length [ISK-1]*INF). Use when IEF $=0$.

CARD 6.7.17 - $1 \mathrm{~B}$

Tells that the variable format specified in card 6.7.18 is to be used for reading card 6.7 20 and refers to this data as the array number specified in card 6.7.19.

CARD 6.7.18 - () - Variable format in parenthesis. This is the format in which the names of materials of variable concentration will be read and printed as heading to printouts of material volume fraction tables etc.

CARD 6.7.19 - $\quad 56 \%$ - The \% sign is used by SWIF to distinguish this array from an integer array (denoted by \$ $\$$ ) and from a floating point array (denoted by **). The name of the materials, as given in card 6.7.20, will be stored in array $56 \%$.

CARD 6.7.20 - Name of material of variable concentration (length ISK). See card 6.7.17 for explanation. The format is as specified in card 6.7.18.

CARD 6.7.21 - $65 \$ \$$ - Defines the zone number for which the ratio $\mathrm{H} / \mathrm{L}$ needs to be calculated. Should be used only for IRATIO $=1$ :

1. 2 (for internal use by SWIF)

2. 1 (for internal use by SWIF)

3. $-\mathrm{N}$ where $\mathrm{N}$ is the zone number corresponding to the location of the first adjoint source $(\mathrm{H})$.

4. $\quad-\mathrm{M}$ where $\mathrm{M}$ is the zone number corresponding to the location of the $2^{\text {nd }}$ adjoint source (L).

5 to 20 . Fill by 0 .

CARD 6.7.22 - T

Terminates SWIF input data segment 4.

CARD 6.7.23 - $\quad \mathrm{T}$

Terminates all the input for SWIF.

\subsection{SWIF COMPOSITION INPUT (11** ARRAY)}

This data block specifies the input arrays to SWIF that are varied by DORIF from iteration to iteration.

CARD 6.8.1 - $\quad($ ) +22 (See Fig. 6.1)

All the following data will be inserted on logical unit 22. Note that the third space is blank.

CARD 6.8.2 - $\quad 11^{* *}$ - current volume fraction of all the materials (length IZM*ISD). 
CARD 6.8.3 - $\quad 42 \mathrm{X}$

Switch reading the input from logical unit 42 starting from the end point reached in the previous reading from logical unit 42 .

\subsection{DORIF COMPOSITION INPUT (12** ARRAY)}

This data block specifies the input arrays to DORT that are varied by DORIF from iteration to iteration.

CARD 6.9.1 - $\quad()+21 \quad$ (See Fig. 6.1)

All the following data will be inserted on logical unit 21. Note that the third space is blank.

CARD 6.9.2 - 12** - DENS(MIX) current volume fraction of all the materials (length MIXL).

CARD 6.9.3 - $\quad 41 \mathrm{x}$

Switch reading the input from logical unit 41 starting from the end point reached in the previous reading from logical unit 41 . 


\subsection{INPUT SAMPLE}

The sample problem models a sphere of radius $65 \mathrm{~cm}$ in RZ geometry using a mesh of $1 \mathrm{~cm}$. The $65 \times 65$ cells are divided into 20 zones. The central $1 \mathrm{cmx} 1 \mathrm{~cm}$ zone is a vacuum in which the direct source is specified. This source zone is surrounded by another vacuum zone that is $4 \mathrm{~cm}$ thick. The following $30 \mathrm{~cm}$ thick spherical shell represents the Beam Shaping Assembly (BSA) of a Boron Neutron Capture Therapy (BNCT) facility. Its composition is to be optimized. It is divided into 12 equal thickness zones. The next $10 \mathrm{~cm}$ shell contains a low concentration of ${ }^{6} \mathrm{LiF}$. The last $20 \mathrm{~cm}$ shell represents the brain to be treated; it is divided into four zones: the first $1 \mathrm{~cm}$ thick zone represents the head outer surface; it is where $\mathrm{L}$ is measured. The following $7 \mathrm{~cm}$ are normal tissue. It is followed by $1 \mathrm{~cm}$ thick tumor region; it is in this region that $\mathrm{H}$ is measured. The remaining $11 \mathrm{~cm}$ thick zone represents normal tissue. The last zone is vacuum that fills the space between the outer "head" surface and a cylinder that is $65 \mathrm{~cm}$ in diameter and $65 \mathrm{~cm}$ high. The system "map" as printed by DORT is shown in Section 6.11 below. Following this map is a list of the zones composition and volume.

The neutron source is that obtained when $2.5 \mathrm{Mev}$ protons impinge on a ${ }^{7} \mathrm{Li}$ target. It is normalized to 1 neutron per second. The DABL-69 multi-group cross-section library (See Table 8.1 for the group structure) and the $\mathrm{S}_{8}-\mathrm{P}_{3}$ transport approximation are used for the DORT calculations.

Many comment statements are introduced in the sample input file so as to help the user to follow this relatively long input specification. The comment cards are denoted in their first location by the following characters:

/ Stands for a comment in the input of DORT. Any data after this sign until the end of card is neglected by the program.

( Stands for the same thing for SWIF.

" Stands for the title card for DORT input; see card 6.2.2.

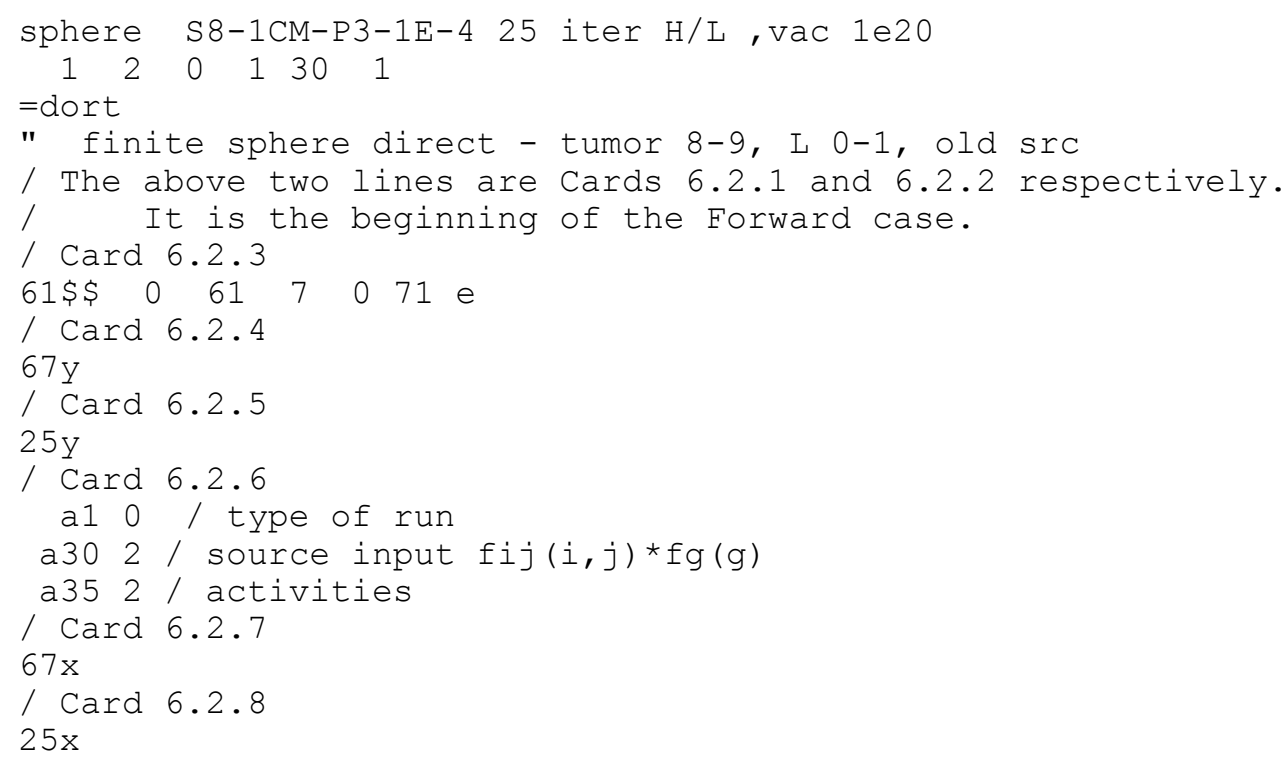


/ Card 6.2.9

a2 1.0 / normalization * width

/ Card 6.2.10

$25 \mathrm{x}$

/ Card 6.2.11

$/$ profile

$25 x$

/ act 1,2 - total tissue/tumor in ALL groups

/ Card 6.2.12

$25 \$ \$-51-52$

/ Card 6.2.13

$26 \$ \$ 4$

/ Card 6.2.14

$\begin{array}{lll}27 * * & 1 & 1\end{array}$

/ Card 6.2.15

t

/ Card 6.2.16

$35 y$

/ point source

/ Card 6.2.17

$96 \star \star 1$ f0 t

/ Card 6.2.19 LAST CARD OF FORWARD CASE

/ "OLD" source $2.5 \mathrm{Mev} \mathrm{p}$ on Li7

$98 * *$

$0.00000 e+00 \quad 0.00000 e+00 \quad 0.00000 e+00 \quad 0.00000 e+00 \quad 0.00000 e+00$

$0.00000 e+00 \quad 0.00000 e+00 \quad 0.00000 e+00 \quad 0.00000 e+00 \quad 0.00000 e+00$

$0.00000 e+00 \quad 0.00000 e+00 \quad 0.00000 e+00 \quad 0.00000 e+00 \quad 0.00000 e+00$

$0.00000 e+00 \quad 0.00000 e+00 \quad 0.00000 e+00 \quad 0.00000 e+00 \quad 0.00000 e+00$

$0.00000 e+00 \quad 0.00000 e+00 \quad 0.00000 e+00 \quad 4.00820 e-02 \quad 1.39214 e-01$

$2.05083 e-013.74583 e-01 \quad 1.28245 e-01 \quad 6.32080 e-02 \quad 2.79300 e-02$

$1.37827 e-02 \quad 2.72520 e-03 \quad 1.42785 e-03 \quad 4.36950 e-04 \quad 1.73130 e-03$

$1.04676 e-03 \quad 3.18075 e-04 \quad 9.76725 e-05 \quad 4.61385 e-05 \quad 2.61090 e-05$

$1.08416 e-052.75190 e-061.14270 e-06 \quad 2.90055 e-07 \quad 1.06697 e-07$

$6.20985 e-080.00000 e+00 \quad 0.00000 e+00 \quad 0.00000 e+00 \quad 0.00000 e+00$

$0.00000 e+00 \quad 0.00000 e+00 \quad 0.00000 e+00 \quad 0.00000 e+00 \quad 0.00000 e+00$

$0.00000 e+00 \quad 0.00000 e+00 \quad 0.00000 e+00 \quad 0.00000 e+00 \quad 0.00000 e+00$

$0.00000 e+00 \quad 0.00000 e+00 \quad 0.00000 e+00 \quad 0.00000 e+00 \quad 0.00000 e+00$

$0.00000 e+00 \quad 0.00000 e+00 \quad 0.00000 e+00 \quad 0.00000 e+00$

$t$

$=$ dort

" sphere adjoint - kerma factor of $\mathrm{H}=8-9 \mathrm{~cm}$ (zone 18)

/ The above two lines are Cards 6.2.1 and 6.2.2 respectively.

/ It is the beginning of the First adjoint-H case.

/ Card 6.2.3

$61 \$ \$ 0628071$ e

/ Card 6.2.4

$67 \mathrm{x}$

/ Card 6.2.5

$25 y$

/ Card 6.2.6

al 1 / type of solution

a30 2 / source input fij(i,j)*fg(g)

/ Card 6.2.7

$67 x$

/ Card 6.2.8

$25 \mathrm{x}$

/ Card 6.2.9

a2 0.0 / No normalization

/ Card 6.2.10

$25 x$ 


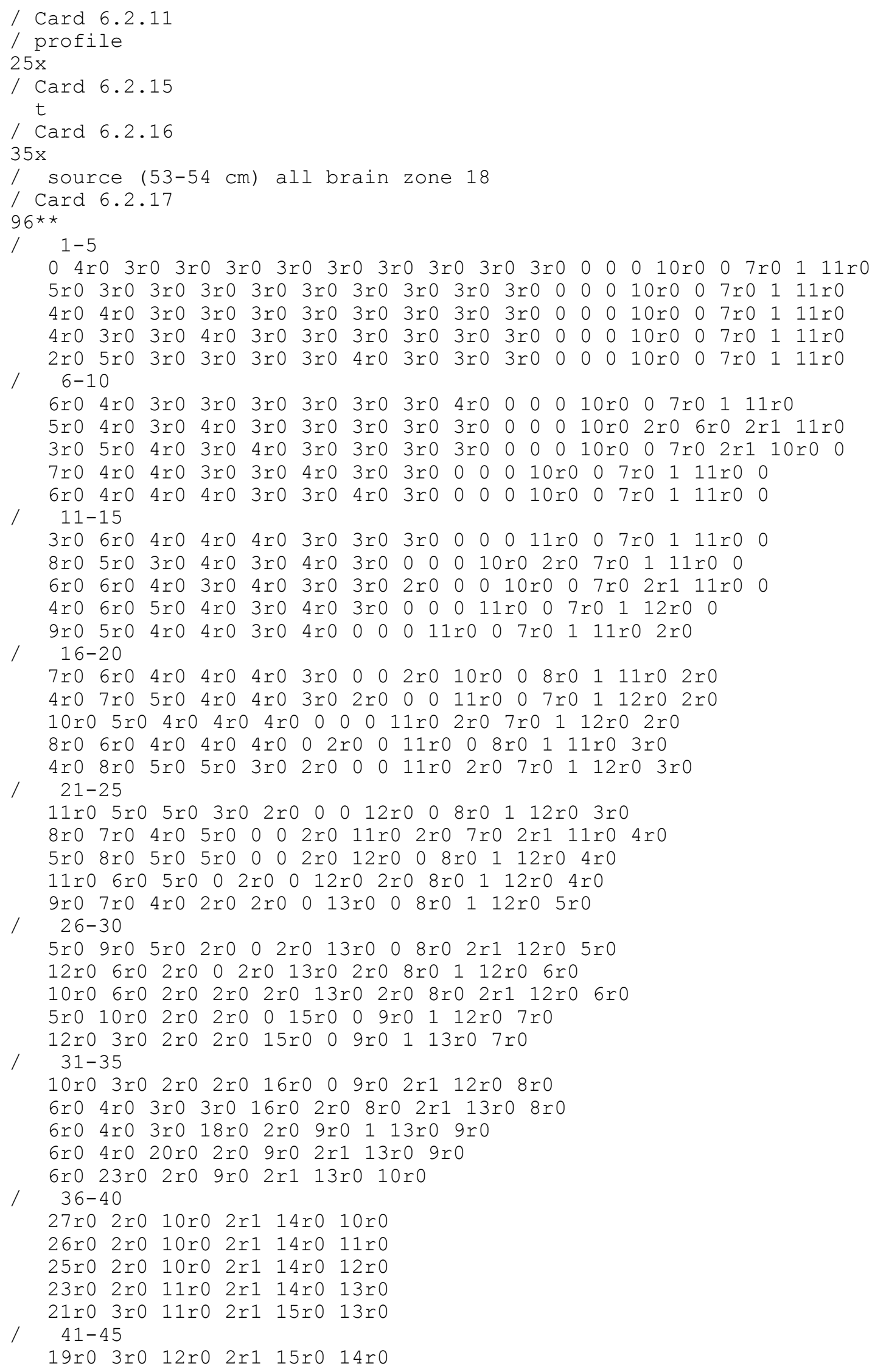




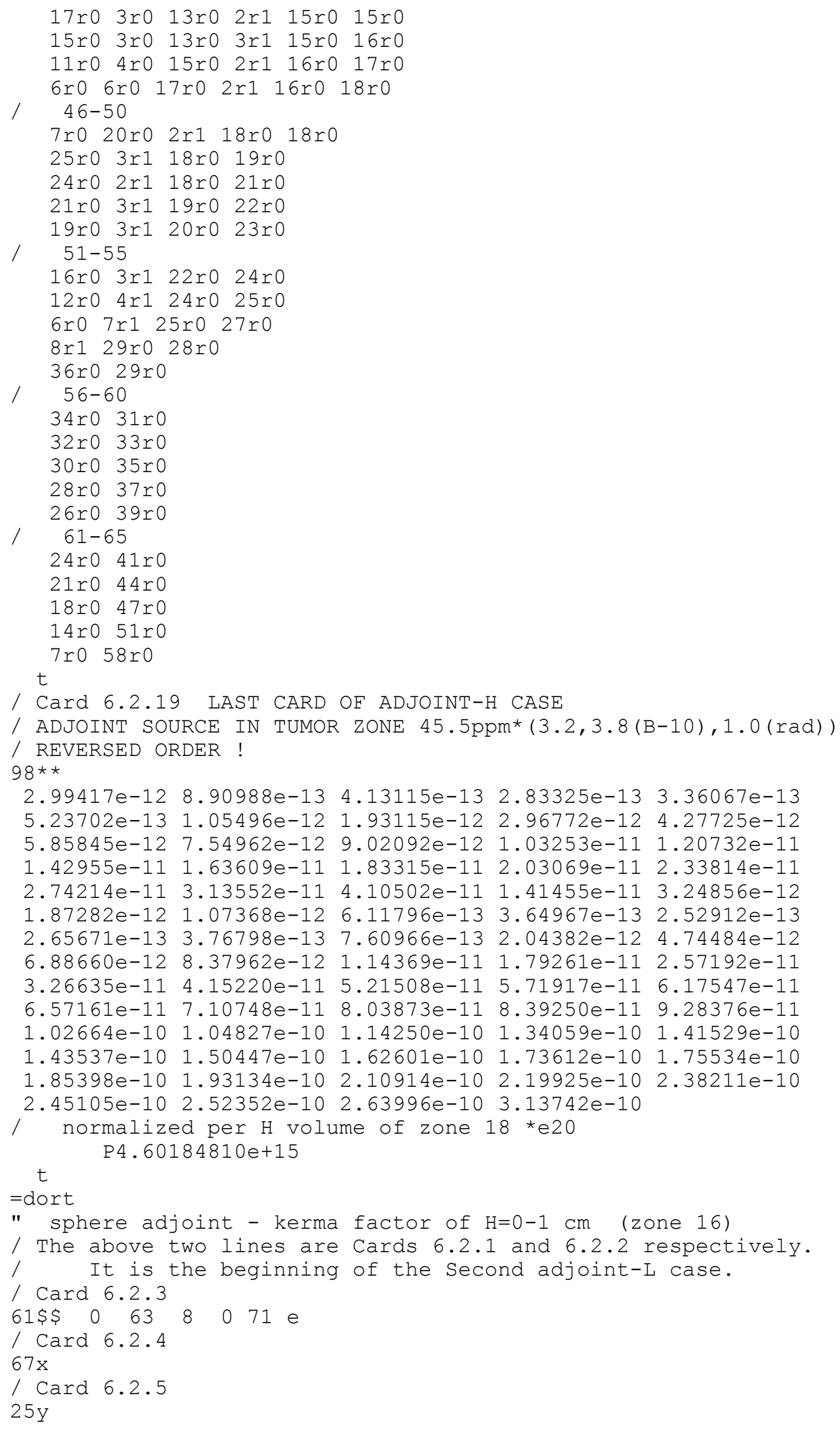


/ Card 6.2.6

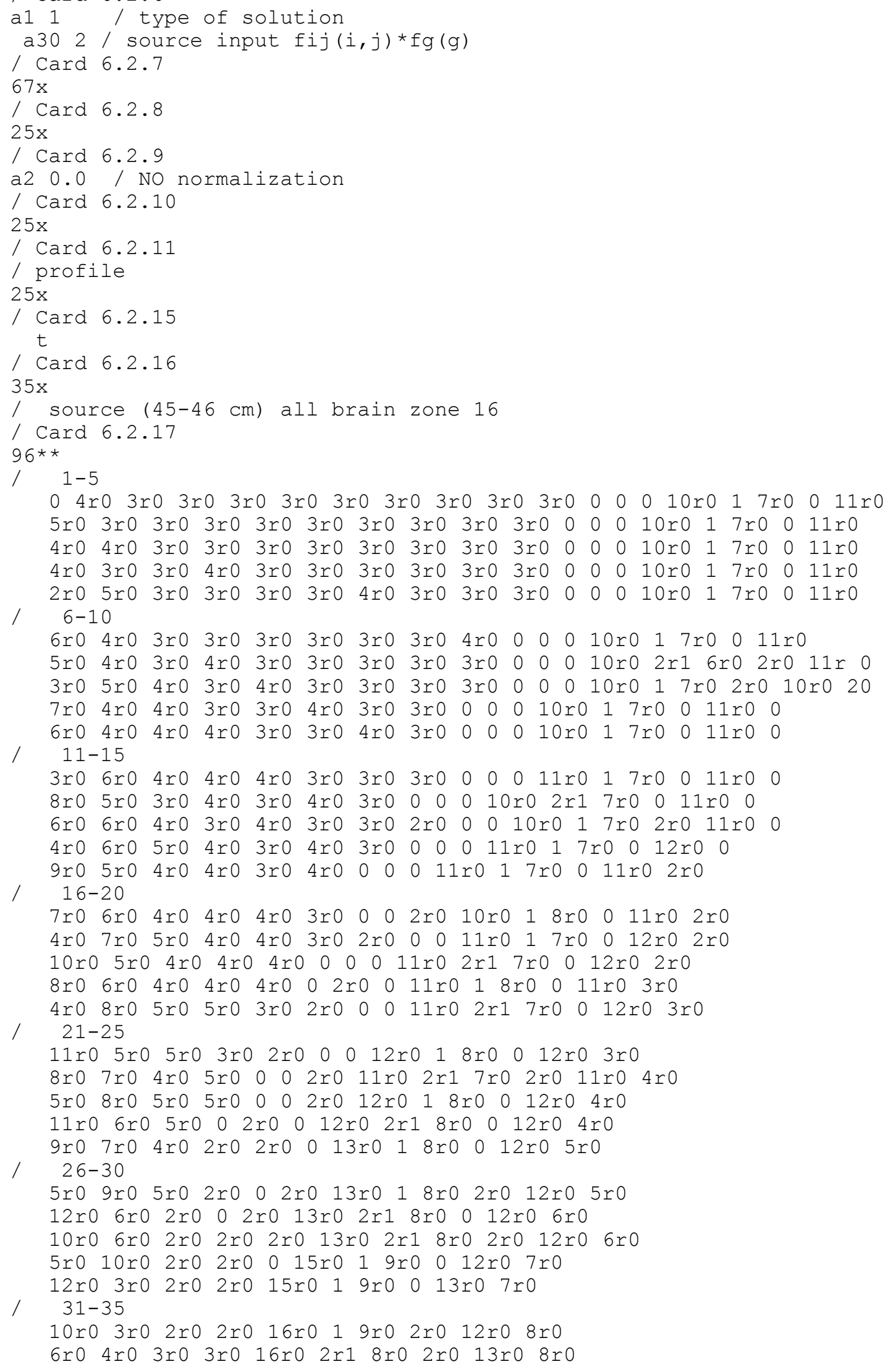




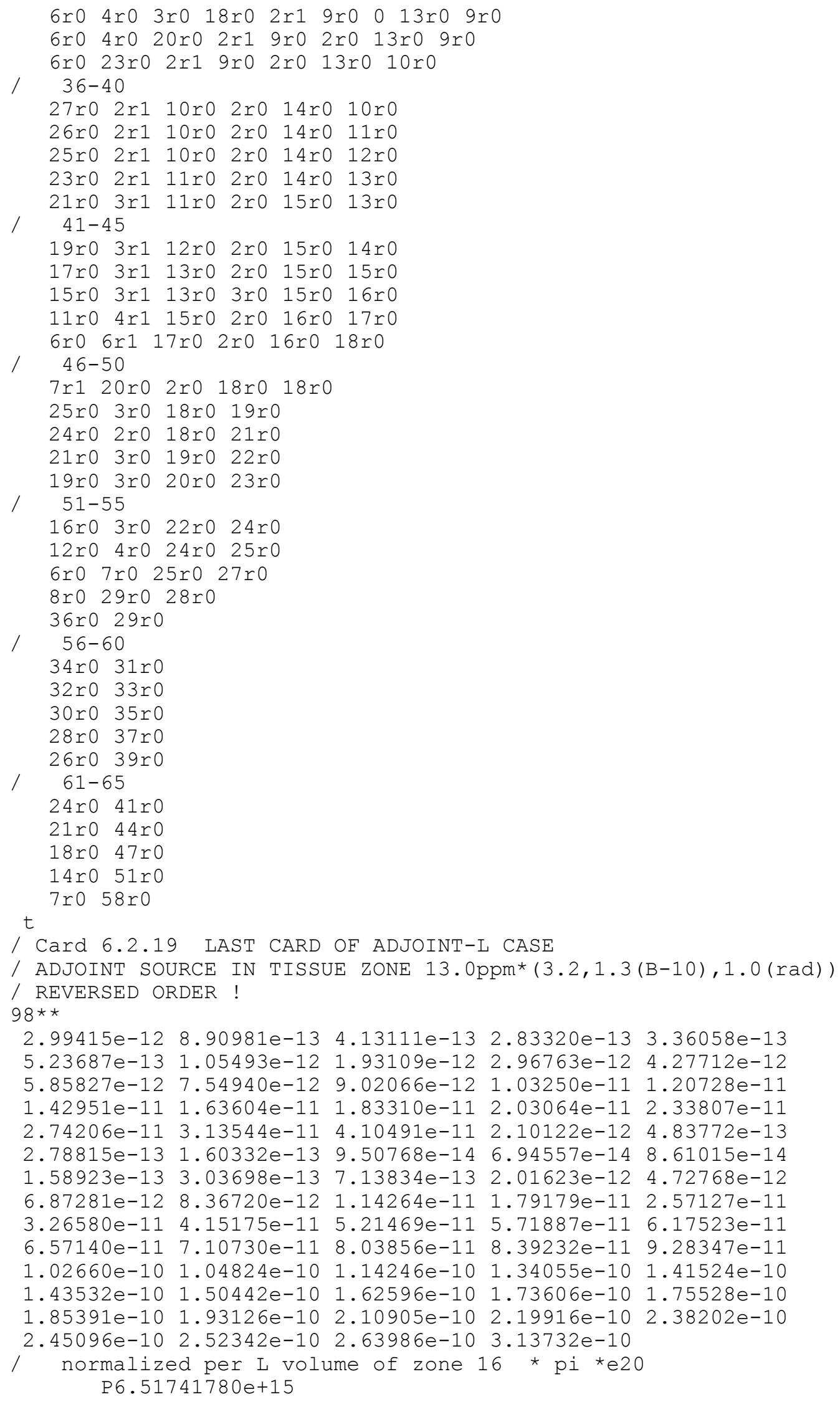




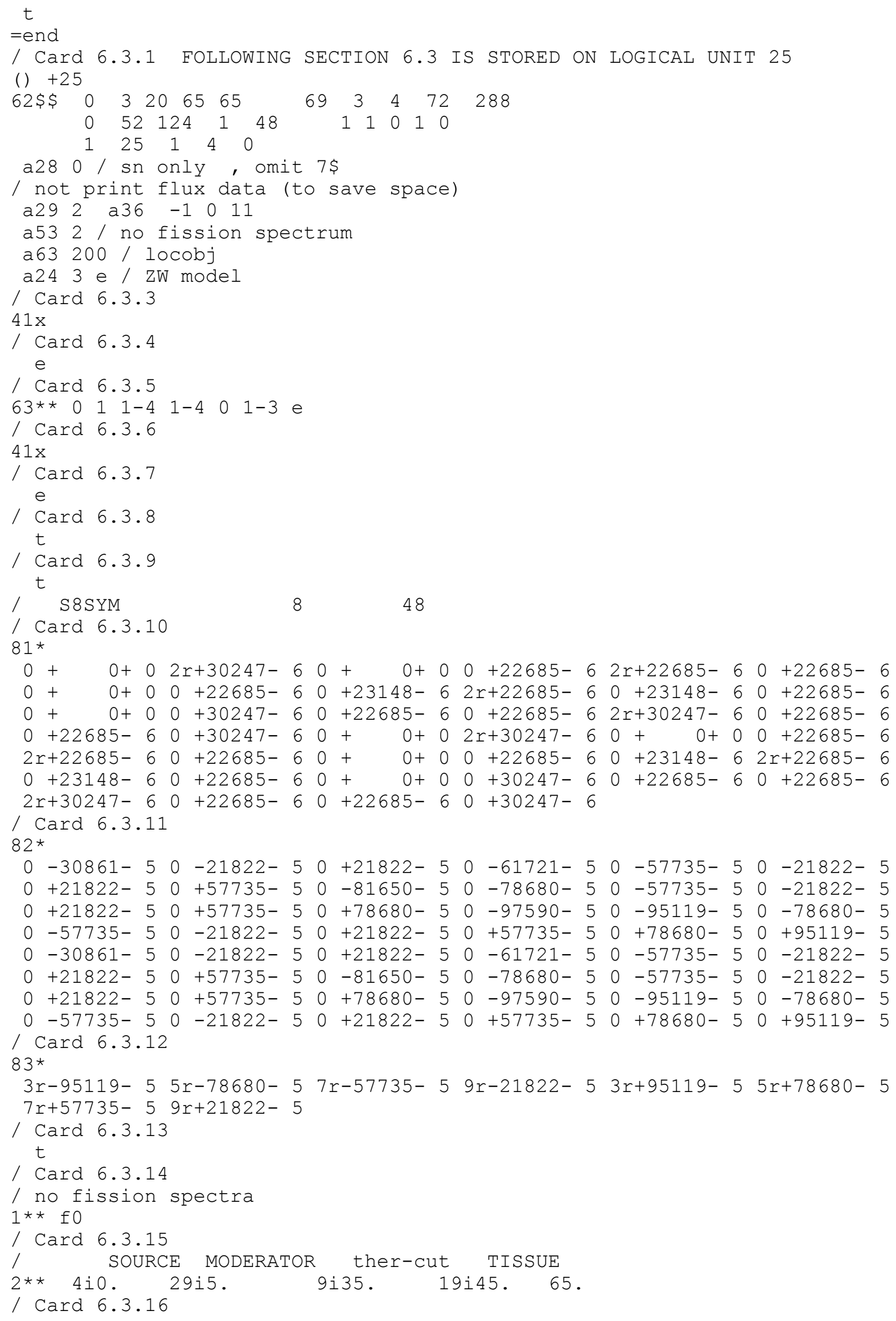




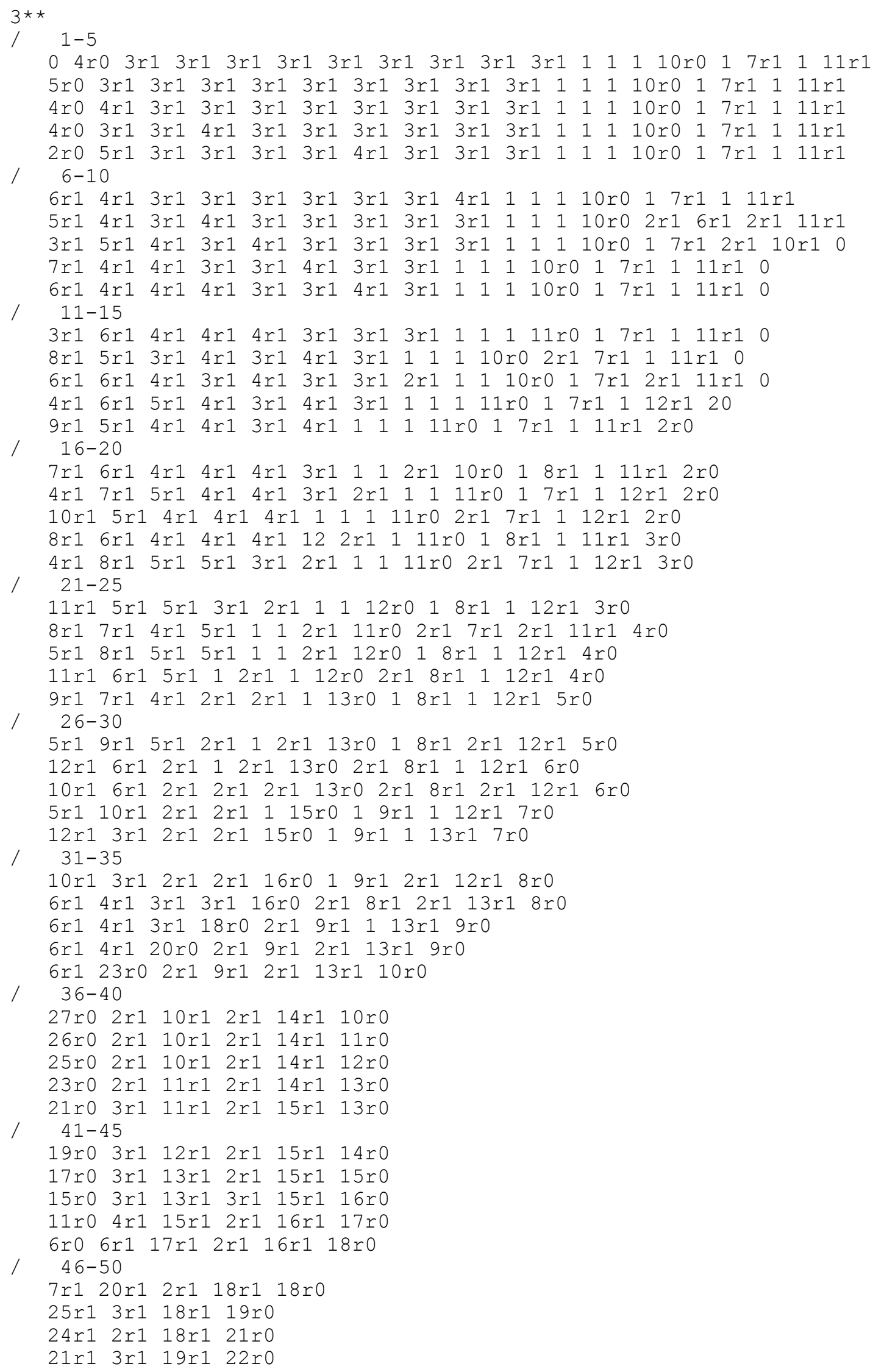




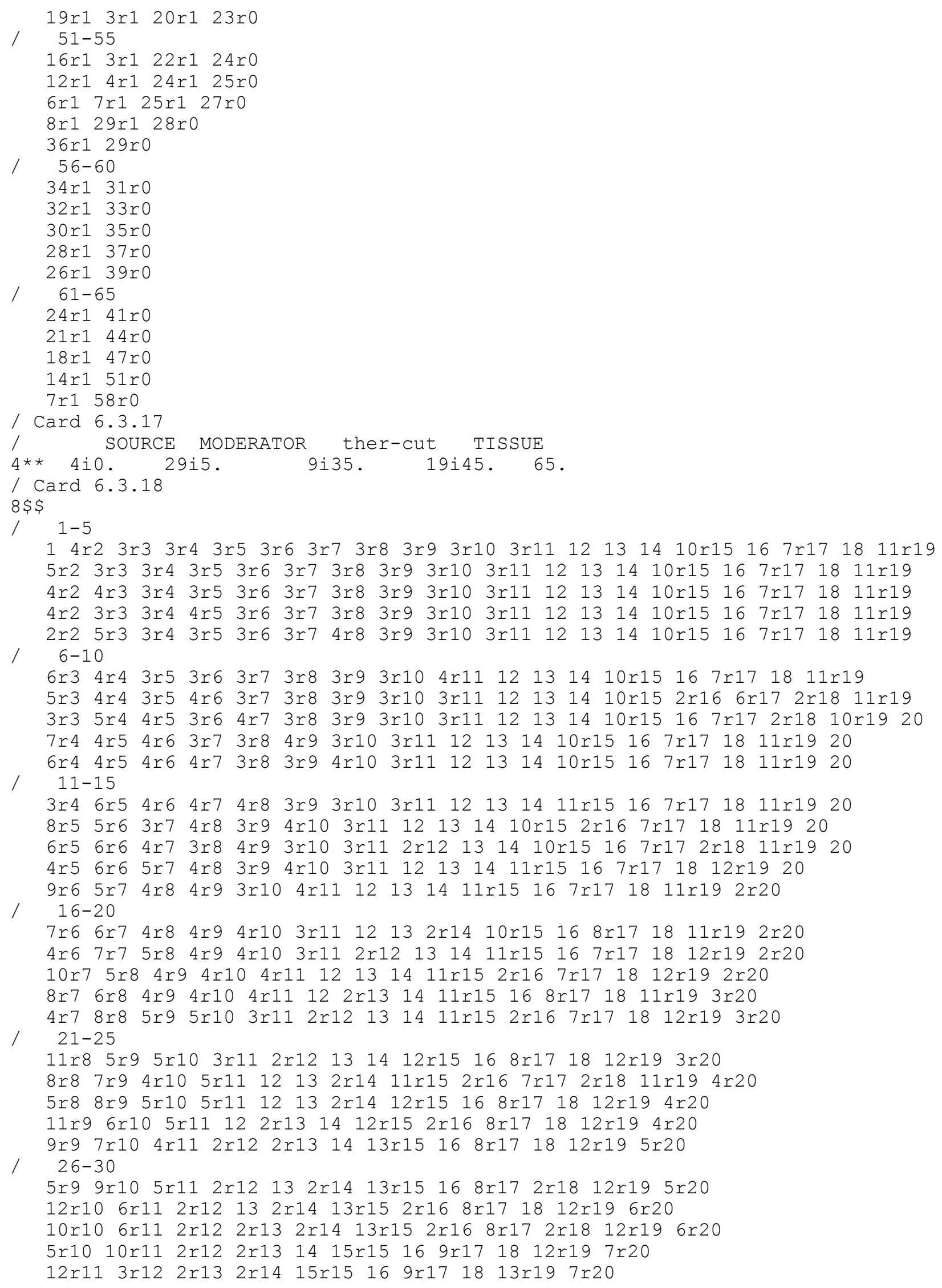




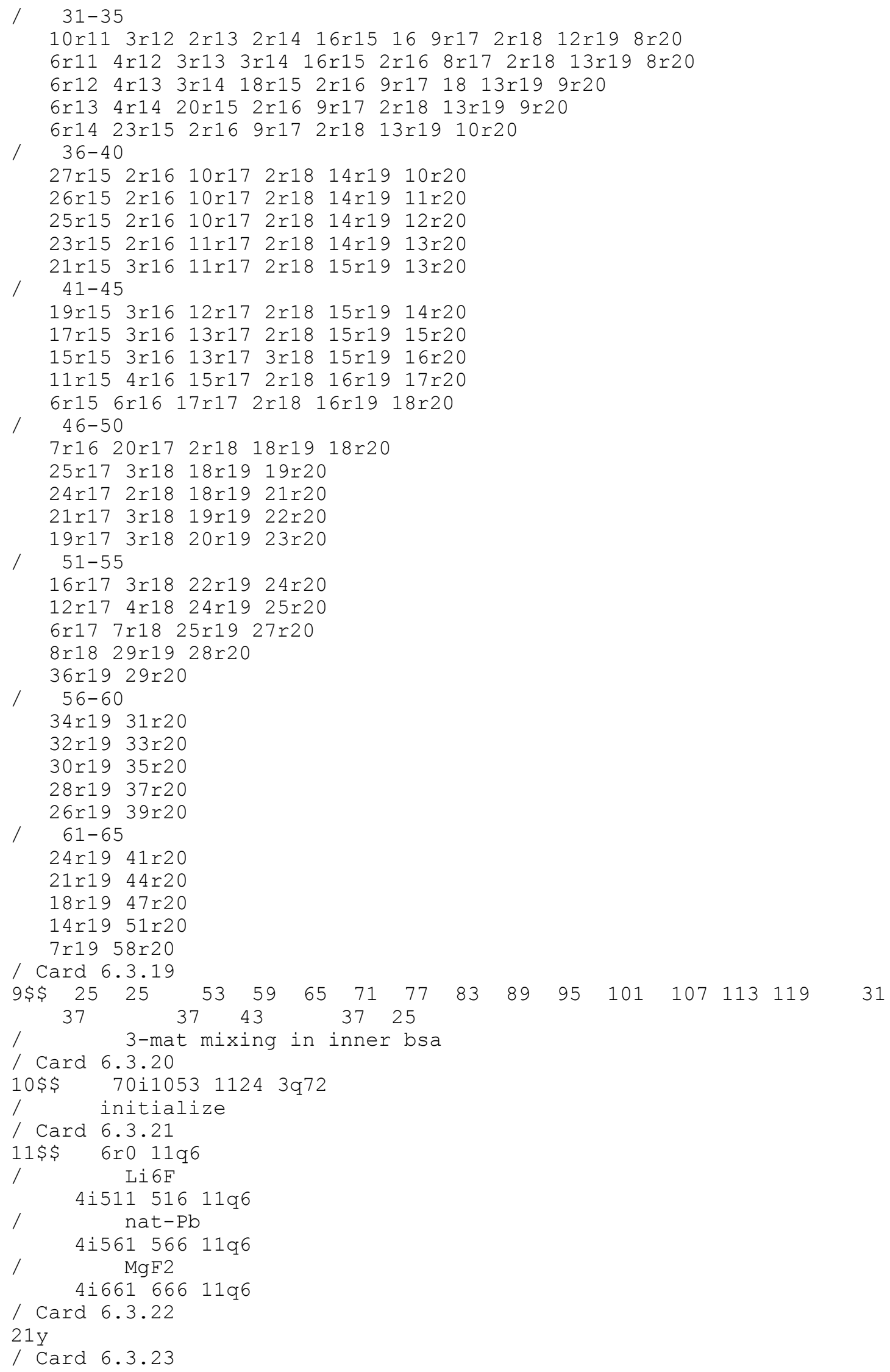




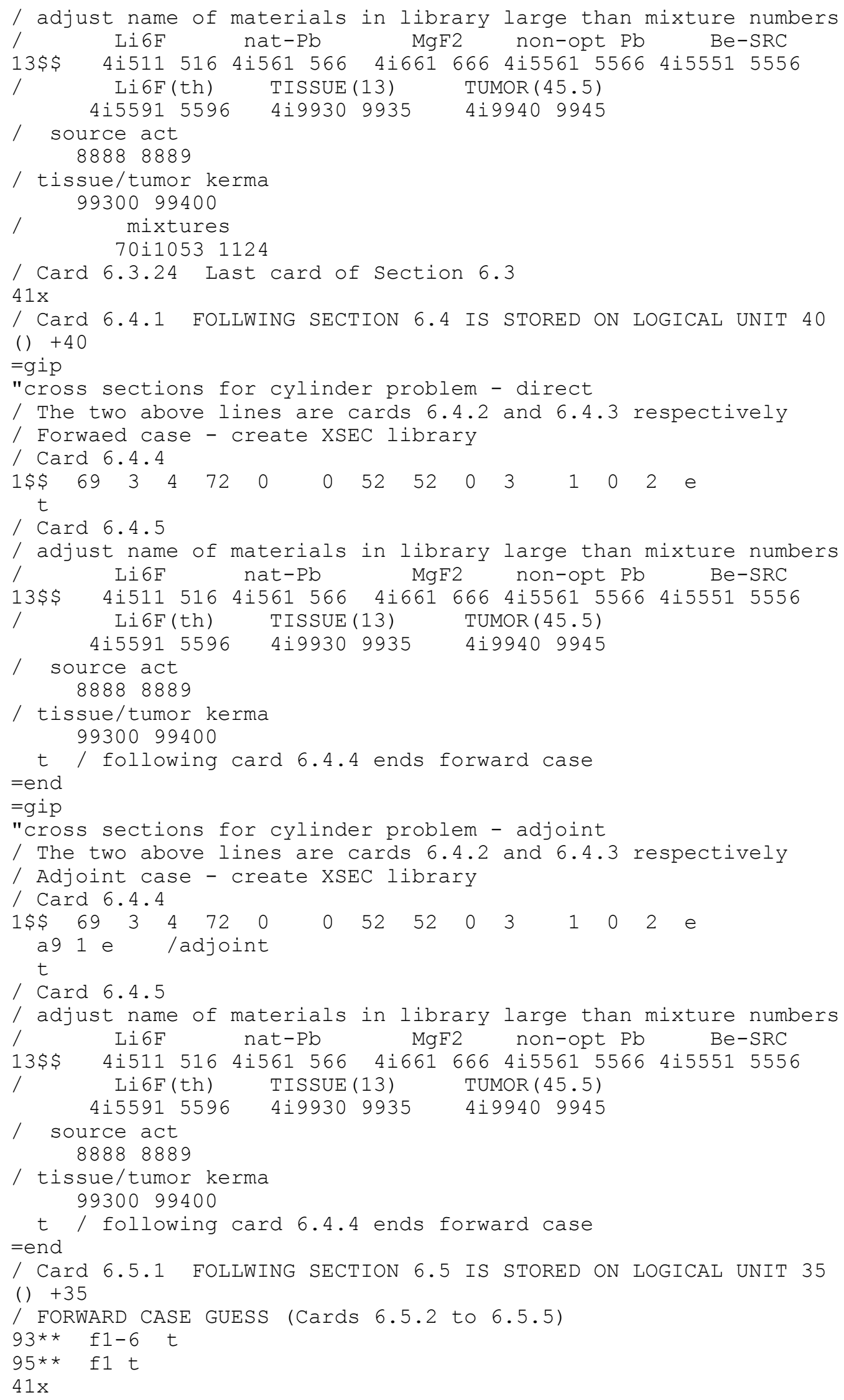




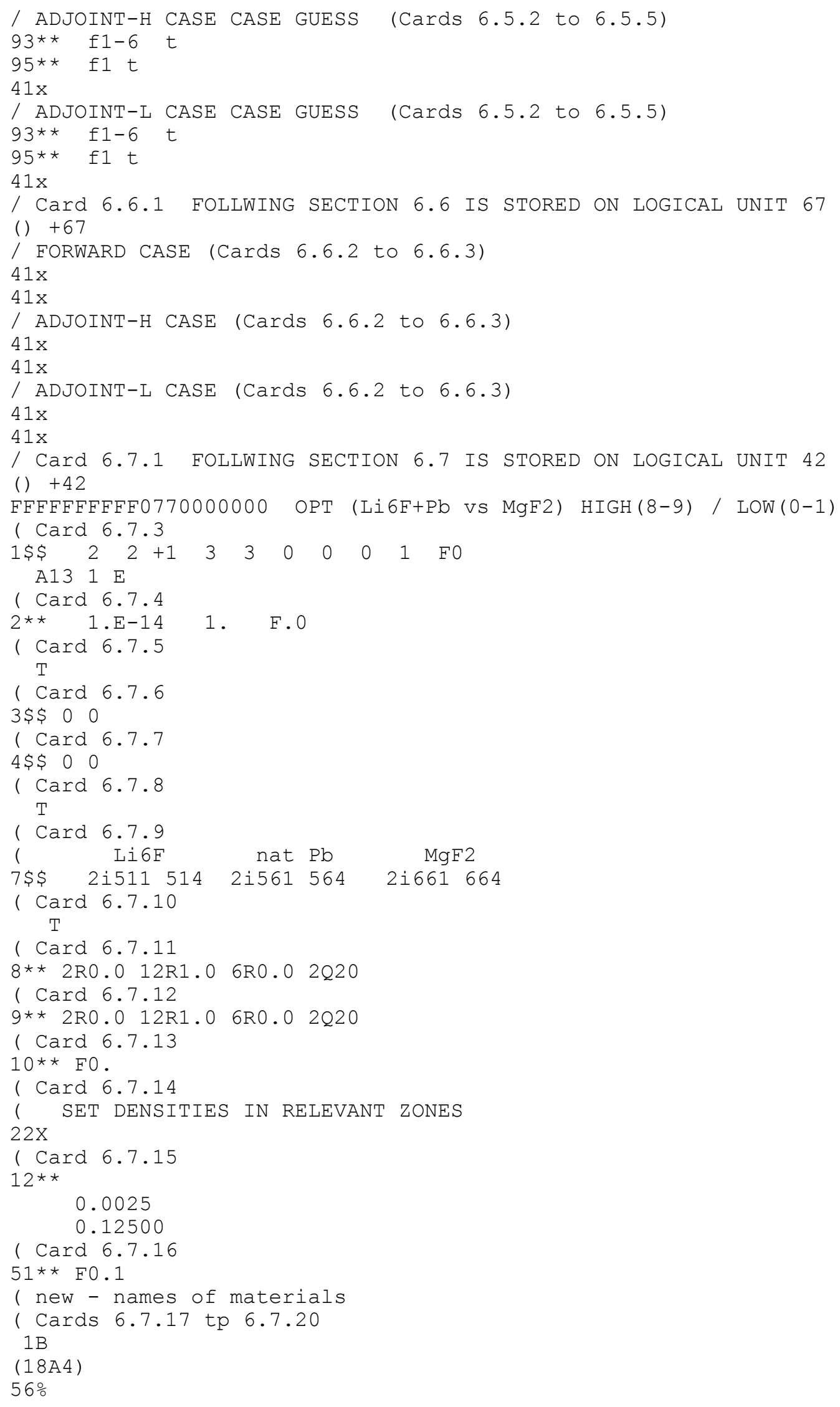




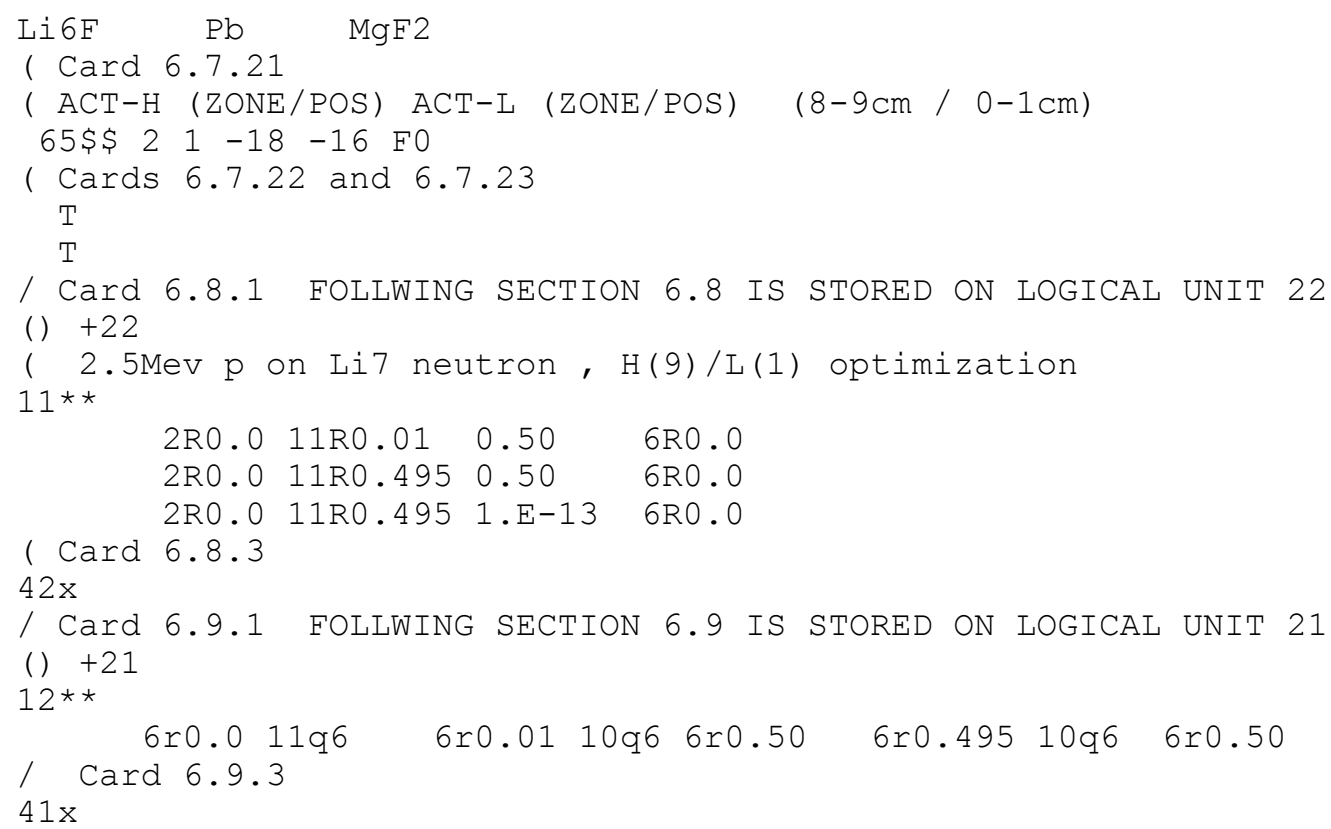

\subsection{OUTPUT SAMPLE}

A small fraction of the DORIF output is provided below. It includes the system "map" as printed by DORT, initial system composition and output of the values of $\mathrm{L}, \mathrm{H}$ and the $\mathrm{H} / \mathrm{L}$ ratio for the first 30 iterations of the optimization process.

Ozone number by interval

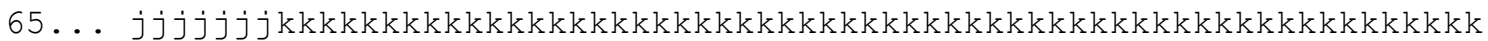

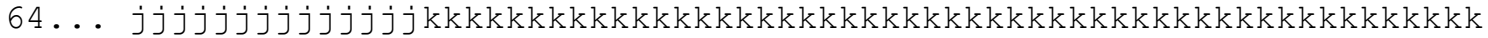

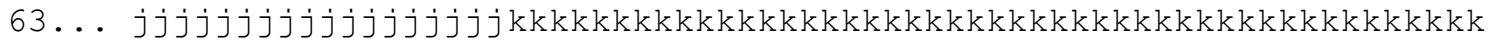

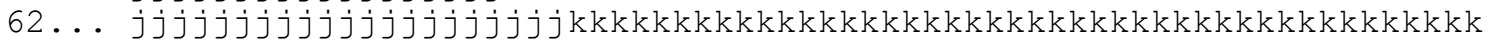

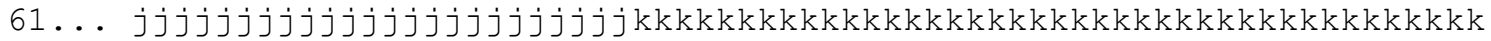

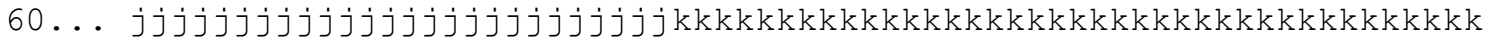

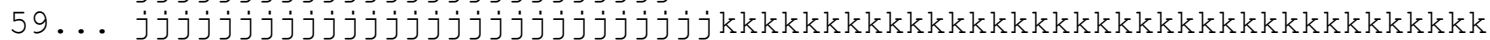
$58 \ldots$... . . . $57 \ldots$...

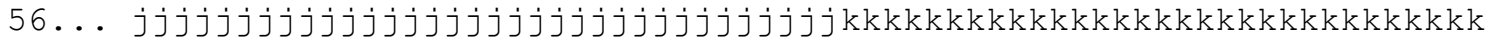

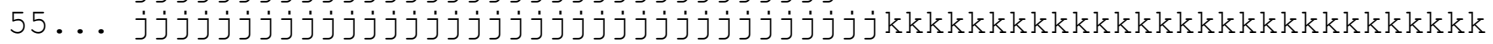

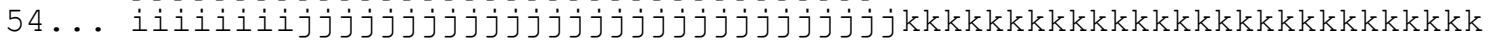
53... hhhhhhiiiiiiijjjjjjjjjjjjjjjjjjjjjjjjjkkkkkkkkkkkkkkkkkkkkkkkkkkk

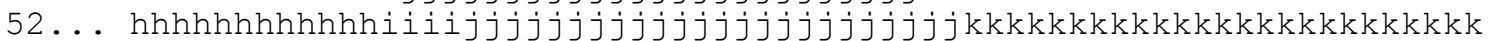

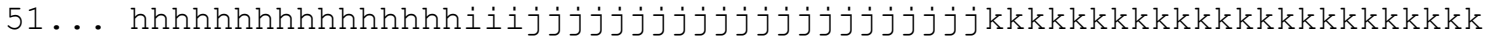

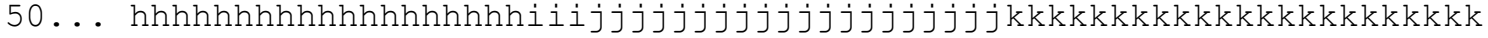

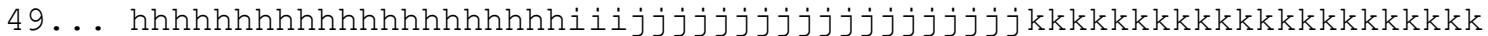

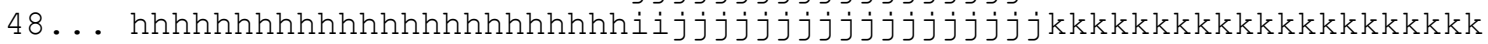

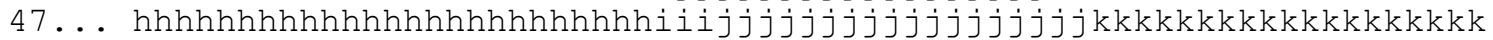
46... ggggggghhhhhhhhhhhhhhhhhhhhiijjjjjjjjjjjjjjjjjjkkkkkkkkkkkkkkkkkk 45... ffffffgggggghhhhhhhhhhhhhhhhhiijjjjjjjjjjjjjjjjkkkkkkkkkkkkkkkkkk $44 \ldots$ fffffffffffgggghhhhhhhhhhhhhhhiijjjjjjjjjjjjjjjjkkkkkkkkkkkkkkkkk 43... fffffffffffffffggghhhhhhhhhhhhhiiijjjjjjjjjjjjjjjkkkkkkkkkkkkkkkk 42... fffffffffffffffffggghhhhhhhhhhhhhiijjjjjjjjjjjjjjjkkkkkkkkkkkkkkk 41... fffffffffffffffffffggghhhhhhhhhhhhiijjjjjjjjjjjjjjjkkkkkkkkkkkkkk 
40... fffffffffffffffffffffggghhhhhhhhhhhiijjjjjjjjjjjjjjjkkkkkkkkkkkkk 39... fffffffffffffffffffffffgghhhhhhhhhhhiijjjjjjjjjjjjjjkkkkkkkkkkkkk 38... fffffffffffffffffffffffffgghhhhhhhhhhiijjjjjjjjjjjjjjkkkkkkkkkkkk 37... ffffffffffffffffffffffffffffgghhhhhhhhhhiijjjjjjjjjjjjjjkkkkkkkkkkk 36... fffffffffffffffffffffffffffgghhhhhhhhhhiijjjjjjjjjjjjjjkkkkkkkkkk 35... eeeeeefffffffffffffffffffffffgghhhhhhhhhiijjjjjjjjjjjjjkkkkkkkkkk 34... ddddddeeeefffffffffffffffffffffgghhhhhhhhhiijjjjjjjjjjjjjkkkkkkkkk 33... ccccccddddeeeffffffffffffffffffgghhhhhhhhhijjjjjjjjjjjjjkkkkkkkkk 32... bbbbbbccccdddeeeffffffffffffffffgghhhhhhhhiijjjjjjjjjjjjjkkkkkkkk $31 \ldots$ bbbbbbbbbbcccddeeffffffffffffffffghhhhhhhhhiijjjjjjjjjjjjkkkkkkkk $30 \ldots$ bbbbbbbbbbbbcccddeefffffffffffffffghhhhhhhhhijjjjjjjjjjjjjkkkkkkk 29... a aaaabbbbbbbbbbccddefffffffffffffffghhhhhhhhhijjjjjjjjjjjjkkkkkkk 28... aaaaaaaaabbbbbbccddeefffffffffffffgghhhhhhhhiijjjjjjjjjjjjkkkkkk 27... aaaaaaaaaaabbbbbbccdeefffffffffffffgghhhhhhhhijjjjjjjjjjjjkkkkkk 26... 99999aaaaaaaabbbbbccdeefffffffffffffghhhhhhhhiijjjjjjjjjjjjkkkkk 25... 999999999aaaaaaabbbbccddefffffffffffffghhhhhhhhijjjjjjjjjjjjkkkkk 24... 99999999999aaaaaabbbbbcddeffffffffffffgghhhhhhhhijjjjjjjjjjjjkkkk 23... 8888899999999aaaaabbbbbcdeeffffffffffffghhhhhhhhijjjjjjjjjjjjkkkk $22 \ldots 888888889999999 a a a a b b b b b c d e e f f f f f f f f f f f g g h h h h h h h i i j j j j j j j j j j j k k k k$ $21 \ldots 8888888888899999 a a a a a b b b c c d e f f f f f f f f f f f f g h h h h h h h h i j j j j j j j j j j j j k k k$ $20 \ldots 77778888888899999 a a a a a b b b c c d e f f f f f f f f f f f g g h h h h h h h i j j j j j j j j j j j j k k k$ $19 \ldots 777777778888889999 a a a a b b b b c d d e f f f f f f f f f f f g h h h h h h h h i j j j j j j j j j j j k k k$ $18 \ldots 7777777777888889999 a a a a b b b b c d e f f f f f f f f f f f g g h h h h h h h i j j j j j j j j j j j j k k$ $17 \ldots 66667777777888889999 a a a a b b b c c d e f f f f f f f f f f f g h h h h h h h i j j j j j j j j j j j j k k$ $16 \ldots 666666677777788889999 a a a a b b b c d e e f f f f f f f f f f g h h h h h h h h i j j j j j j j j j j j k k$ $15 \ldots 6666666667777788889999 a a a b b b b c d e f f f f f f f f f f g h h h h h h h i j j j j j j j j j j j k k$ 14... 5555666666777778888999aaaabbbcdefffffffffffghhhhhhhijjjjjjjjjjjjk

$13 \ldots 55555566666677778889999 a a a b b b c c d e f f f f f f f f f f g h h h h h h h i i j j j j j j j j j j j k$

$12 \ldots 55555555666667778888999 a a a a b b b c d e f f f f f f f f f f g g h h h h h h h i j j j j j j j j j j j k$

$11 \ldots 444555555666677778888999 a a a b b b c d e f f f f f f f f f f f g h h h h h h h i j j j j j j j j j j j k$

$10 \ldots 444444555566667777888999 a a a a b b b c d e f f f f f f f f f f g h h h h h h h i j j j j j j j j j j j k$

9... 4444444555566667778889999aaabbbcdeffffffffffghhhhhhhijjjjjjjjjjjk

8... 3334444455556667777888999aaabbbcdeffffffffffghhhhhhhiijjjjjjjjjjk

7... $3333344445556666777888999 a a a b b b c d e f f f f f f f f f f g h h h h h h i i j j j j j j j j j j j$

6... $3333334444555666777888999 a a a b b b b c d e f f f f f f f f f f g h h h h h h i j j j j j j j j j j j$

5... 22333334445556667778888999aabbbcdeffffffffffghhhhhhhijjjjjjjjjjj

4... $22223334445555666777888999 a a a b b b c d e f f f f f f f f f f g h h h h h h h i j j j j j j j j j j j$

3... 22223333444555666777888999aabbbcdeffffffffffghhhhhhhijjjjjjjjjjj

2... $22222333444555666777888999 a a a b b b c d e f f f f f f f f f f g h h h h h h i j j j j j j j j j j j$

$1 \ldots 12222333444555666777888999 a a a b b b c d e f f f f f f f f f f g h h h h h h h i j j j j j j j j j j$ yzt

$\operatorname{xrr} 12345678901234567890123456789012345678901234567890123456789012345$ 00000000011111111112222222222333333333344444444445555555555666666

Osymbol $=\begin{array}{lllllllllllllllllllll}1 & 2 & 3 & 4 & 5 & 6 & 7 & 8 & 9 & \mathrm{a} & \mathrm{b} & \mathrm{c} & \mathrm{d} & \mathrm{e} & \mathrm{f} & \mathrm{g} & \mathrm{h} & \mathrm{i} & \mathrm{j} & \mathrm{k}\end{array}$ Onumber $=\begin{array}{llllllllllllllllllll}1 & 2 & 3 & 4 & 5 & 6 & 7 & 8 & 9 & 10 & 11 & 12 & 13 & 14 & 15 & 16 & 17 & 18 & 19 & 20\end{array}$

0

Omatl number by interval

65 . . 2222222pppppppppppppppppppppppppppppppppppppppppppppppppppppppp 64 .. 22222222222222 2ppppppppppppppppppppppppppppppppppppppppppppppppp 63 . . 222222222222222222ppppppppppppppppppppppppppppppppppppppppppp 62 . . 222222222222222222222ppppppppppppppppppppppppppppppppppppppppp $61 \ldots 2222222222222222222222$... 22 pppppppppppppppppppppppppppppppppppppp $60 \ldots 2222222222222222222222222$. . 2pppppppppppppppppppppppppppppppppppp $59 \ldots 222222222222222222222222222$. . 22 ppppppppppppppppppppppppppppppppp $58 \ldots 222222222222222222222222222222$.. $22 p p p p p p p p p p p p p p p p p p p p p p p p p p p p p$ $57 \ldots 22222222222222222222222222222222$.. 2 ppppppppppppppppppppppppppppppp $56 \ldots 2222222222222222222222222222222222$... 2 ppppppppppppppppppppppppppppp 55 . . . 222222222222222222222222222222222222 pppppppppppppppppppppppppppp $54 \ldots 8888888822222222222222222222222222222$ ppppppppppppppppppppppppppp 
53... 22222288888882222222222222222222222222ppppppppppppppppppppppppp $52 \ldots \quad 2222222222228888222222222222222222222222$ ppppppppppppppppppppppp $51 \ldots 22222222222222228882222222222222222222222$ pppppppppppppppppppppp $50 \ldots 222222222222222222288822222222222222222222$ ppppppppppppppppppppp $49 \ldots$. 2222222222222222222228882222222222222222222 pppppppppppppppppppp $48 \ldots$.. 22222222222222222222222288222222222222222222ppppppppppppppppppp $47 \ldots$.. 2222222222222222222222222888222222222222222222ppppppppppppppppp $46 \ldots$. 22222222222222222222222222288222222222222222222pppppppppppppppp $45 \ldots$ VvVvVv22222222222222222222222882222222222222222 ppppppppppppppppp $44 \ldots$ vvvvVvVvVvv2222222222222222222882222222222222222pppppppppppppppp

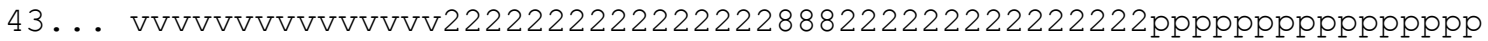

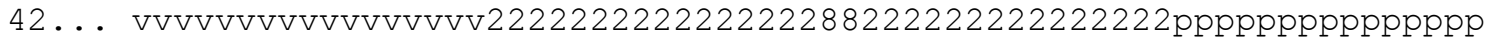
$41 \ldots$ vVvVvVvVvVvVvVvvvvv22222222222222288222222222222222ppppppppppppp

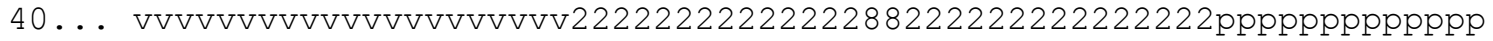

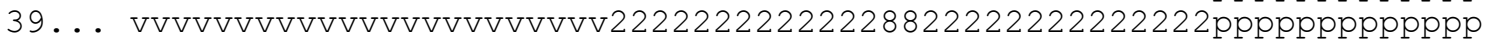

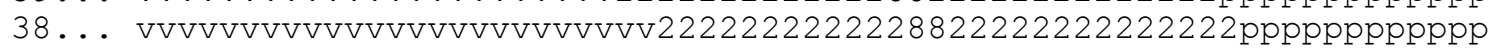

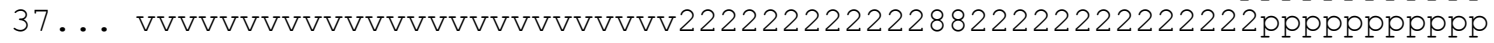
$36 \ldots$ vVVVVVVVVVVVVVVVVVVVVVVVVVv2222222222228822222222222222ppppppppp

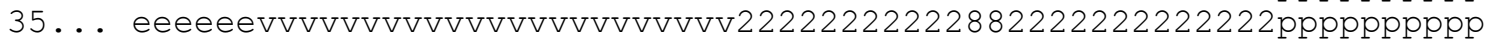

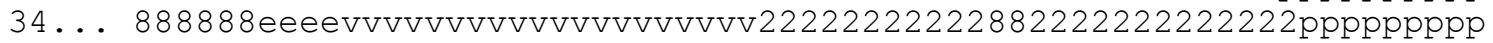
33 ... 2222228888 eeevvvvvvvvvvvvvvvvvv2222222222282222222222222pppppppp

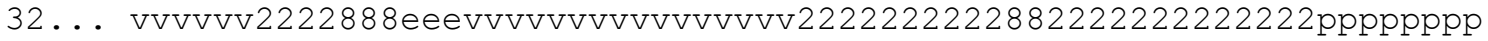

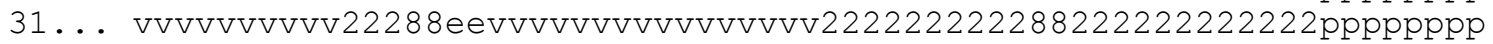

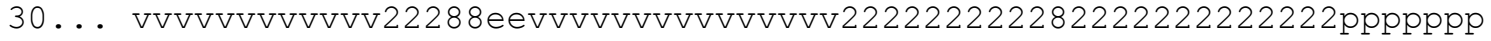

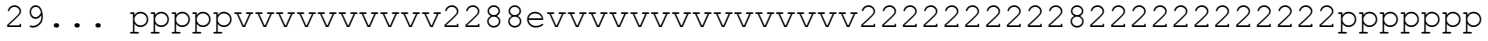

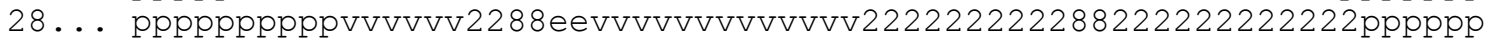

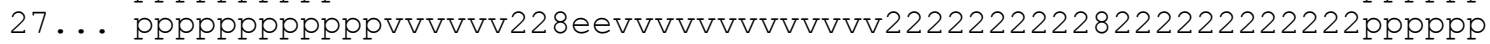

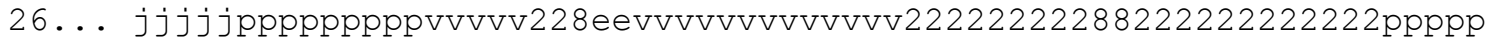

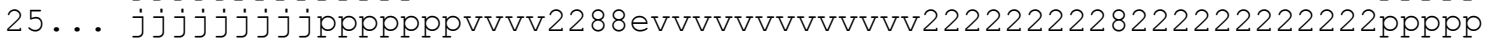

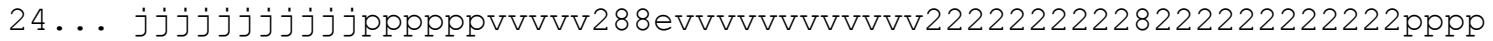
23... dddddjjjjjjjjpppppvvvvv28 eevvvvvvvvvvvv2222222228222222222222pppp $22 \ldots$ ddddddddjjjjjjjppppvvvvv28eevvvvvvvvvvv2222222228822222222222pppp $21 \ldots$ dddddddddddjjjjjpppppvvv228evvvvvvvvvvvv2222222228222222222222ppp $20 \ldots 7777 d d d d d d d d j j j j j$ pppppvvv228evvvvvvvvvvv22222222228222222222222ppp $19 \ldots 77777777 d d d d d d j j j j$ ppppvvvv288evvvvvvvvvvv222222222822222222222ppp $18 \ldots 7777777777 d d d d d j j j j p p p p v v v v 28$ evvvvvvvvvvv2222222228222222222222pp 17... 11117777777dddddjjjjppppvvv228 evvvvvvvvvvv222222228222222222222pp $16 \ldots 111111777777 d d d d j j j j p p p p v v v 28$ eevvvvvvvvvv222222222822222222222pp $15 \ldots 1111111177777 d d d d j j j j p p p v v v v 28$ evvvvvvvvvvv22222222822222222222pp $14 \ldots$ uuuu11111177777ddddjjjppppvvv28evvvvvvvvvvv222222228222222222222p $13 .$. uuuuuu 111117777 dddjjjjpppvvv228 evvvvvvvvvv222222228822222222222p $12 \ldots$ uuuuuuuu11111777ddddjjjppppvvv28 evvvvvvvvvv222222222822222222222p $11 \ldots$ ooouuuuuul1117777ddddjjjpppvvv28 evvvvvvvvvvv22222222822222222222p

$10 \ldots$ o . o00oouuuu11117777dddj jjppppvvv28evvvvvvvvvv22222222822222222222p 9... o00000ouuuu1111777dddjjjjpppvvv28evvvvvvvvvv22222222822222222222p 8... iiiooooouuuu1117777dddjjjpppvvv28evvvvvvvvvv22222222882222222222p

7 ... iiiiioooouuu1111777dddjjjpppvvv28evvvvvvvvvv222222228822222222222 6... iiiiiioooouuu111777dddjjjpppvvvv28 evvvvvvvvvv22222222822222222222 5... ppiiiiiooouuu111777ddddjjjpppvvv28 evvvvvvvvvv22222222822222222222

4 ... ppppiiiooouuuu111777dddjjjpppvvv28evvvvvvvvvv22222222822222222222

3... ppppiiiiooouuu111777dddjjjpppvvv28evvvvvvvvvv22222222822222222222

2... pppppiiiooouuu111777dddjjjpppvvv28evvvvvvvvvv22222222822222222222

1... pppppiiiooouuu111777dddjjjpppvvv28evvvvvvvvvv22222222822222222222 yzt

xrr 12345678901234567890123456789012345678901234567890123456789012345 00000000011111111112222222222333333333344444444445555555555666666

0symbol $=\begin{array}{lllllllllllllllllllllll}1 & 2 & 3 & 4 & 5 & 6 & 7 & 8 & 9 & a & b & c & d & e & f & g & h & i & j & k & l & m & n\end{array}$

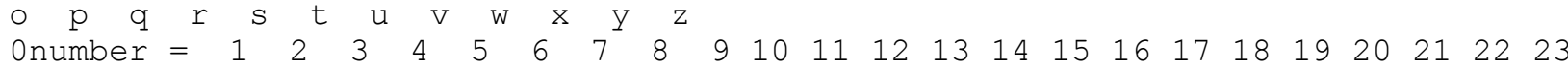
$\begin{array}{lllllllllllll}24 & 25 & 26 & 27 & 28 & 29 & 30 & 31 & 32 & 33 & 34 & 35\end{array}$ 


$\begin{array}{llll}24 & 2.9943 \mathrm{E}-16 & 3.4288 \mathrm{E}-16 & 1.1451 \mathrm{E}+00 \\ 25 & 2.9578 \mathrm{E}-16 & 3.3858 \mathrm{E}-16 & 1.1447 \mathrm{E}+00 \\ 26 & 2.9225 \mathrm{E}-16 & 3.3442 \mathrm{E}-16 & 1.1443 \mathrm{E}+00 \\ 27 & 2.8883 \mathrm{E}-16 & 3.3040 \mathrm{E}-16 & 1.1439 \mathrm{E}+00 \\ 28 & 2.8551 \mathrm{E}-16 & 3.2648 \mathrm{E}-16 & 1.1435 \mathrm{E}+00 \\ 29 & 2.8229 \mathrm{E}-16 & 3.2268 \mathrm{E}-16 & 1.1431 \mathrm{E}+00 \\ 30 & 2.7917 \mathrm{E}-16 & 3.1900 \mathrm{E}-16 & 1.1427 \mathrm{E}+00\end{array}$




\section{VALIDITY TESTS}

The validity of the 2-D SWAN has been checked using a couple of approaches: (a) Comparing the perturbation theory prediction of the effect of small changes in the system composition on a performance parameter of interest against the difference between the performance parameter calculated by DORT for the perturbed and reference systems. (b) Comparing the optimal system composition arrived at using the 2-D SWAN with that of the 1-D SWAN. This comparison was done for an infinite slab and for an infinite cylindrical system. Such systems can be identically modeled by the two codes. In the following we shall describe only the latter test that is an integral test.

The system is a straight cylinder, infinite in the $\mathrm{z}$ direction. The source is at the innermost interval, $1 \mathrm{~cm}$ in radius. Its spectrum is that obtained when $2.5 \mathrm{MeV}$ protons impinge on a ${ }^{7} \mathrm{Li}$ target ${ }^{6}$. The source is surrounded by a $4 \mathrm{~cm}$ of vacuum and then by a $30 \mathrm{~cm}$ thick "beam shaping assembly" (BSA). The BSA consists of $\mathrm{MgF}_{2},{ }^{7} \mathrm{LiF}$ and ${ }^{6} \mathrm{LiF}$. The BSA is surrounded by a $10 \mathrm{~cm}$ thick vacuum layer that is surrounded by a $20 \mathrm{~cm}$ thick tissue. The problem is to find that distribution of the three BSA constituents that will maximize $\mathrm{H} / \mathrm{L}$, where $\mathrm{H}$ is the equivalent dose rate received by a tumor that is located between $8 \mathrm{~cm}$ and $9 \mathrm{~cm}$ inside the tissue and $\mathrm{L}$ is the equivalent dose rate received by the normal tissue in the $1 \mathrm{~cm}$ thick shell closest to the BSA. It is assumed that the ${ }^{10} \mathrm{~B}$ concentration in the tumor and in the normal tissue is, respectively, 45.5 $\mathrm{ppm}$ and $13 \mathrm{ppm}$. It is also assumed that the compound factor is 3.8 for the tumor and 1.3 for the normal tissue. The RBE used is 3.2 for neutrons and 1.0 for photons. The spatial mesh consists of $1 \mathrm{~cm}$ intervals and the BSA is divided into $3 \mathrm{~cm}$ thick radial zones except for the outermost three zones that are $1 \mathrm{~cm}$ thick. The composition within each zone is uniform. The initial composition is $100 \% \mathrm{MgF}_{2}$ except in the outermost zone in which the composition is $100 \%{ }^{6} \mathrm{LiF}$. Axially, the 2-D system is taken to be $20 \mathrm{~cm}$ long and is divided into twenty 1-cm intervals and four $5-\mathrm{cm}$ zones. A reflective boundary condition is imposed at the axial boundaries while a vacuum boundary condition is assumed for the radial boundary. The source intensity is normalized to 1 neutron per $\mathrm{cm}$ of source. The adjoint source is normalized to give the equivalent dose in units of "equivalent Grays". The $\mathrm{S}_{8}-\mathrm{P}_{3}$ approximation is used for solving the transport equation and its adjoints.

Figures 7.1 and 7.2 compare the optimal composition arrived at by the new 2-D SWAN code (also referred to as DORIF), versus the optimal composition arrived at with the 1-D SWAN $\operatorname{code}^{2}$. The 1-D SWAN code has been in use for many years and for many applications (See, for example, the bibliography at the end of reference 6) and is assumed to be reliable. In each zone, $\mathrm{MgF}_{2}$ makes the difference between the sum of the volume fractions in Figures 7.1 and 7.2 and between 1.0. The agreement between the two codes is good; the differences in the ${ }^{6} \mathrm{LiF}$ concentrations (Figure 7.2) are all in the below $1 \%$ concentration level. Most of the ${ }^{6} \mathrm{LiF}$ is called for the last BSA zone and there the two codes call for identical concentration.

Figures 7.3 through 7.5 compare the evolution, with the number of iterations, of three performance parameters: $\mathrm{H}, \mathrm{L}$ and their ratio. The convergence pattern of the 2-D code is very similar to that of the reference 1-D SWAN. It takes only 5 iterations to approach the plateau in $\mathrm{H} / \mathrm{L}$. After 20 iterations the H/L ratio is very close to its asymptotic value. This is despite of the 
fact that both the $\mathrm{H}$ and $\mathrm{L}$ values keep drifting downwards (See figures 7.3 and 7.4) with increasing number of iterations.

Figure 7.6 shows the EVREF for replacing $\mathrm{MgF}_{2}$ by ${ }^{6} \mathrm{LiF}$ corresponding to the last couple of iterations. It is observed that the EVREF changes sign from negative (hollow symbol) in iteration 1 to positive (filled symbol) in iteration 2. Iteration 1 and 2 are two consecutive iterations after convergence has been reached. A negative EVREF calls for removal, whereas a positive EVREF calls for the addition of some ${ }^{6} \mathrm{LiF}$. The ${ }^{6} \mathrm{LiF}$ density change going from iteration 1 to iteration 2 is very small; its effect on the value of $\mathrm{H} / \mathrm{L}$ is not detectable in Figure 6. When such a switching of EVREF signs occur, we know for sure that the optimal concentration has been reached.

Not observable in Figure 7.6 is the fact that the EVREF in the outermost zone is positive both in iteration 1 and 2. A positive EVREF implies that more ${ }^{6} \mathrm{LiF}$ is called for. However, the ${ }^{6} \mathrm{LiF}$ volume fraction in the last zone is already 1.0 (See Figure 7.2).

Figures 7.7 and 7.8 illustrate the evolution of the concentration of ${ }^{6} \mathrm{LiF}$ and of ${ }^{7} \mathrm{LiF}$ in the iterative optimization process. The initial concentration of both materials is zero, except for the last zone in which the ${ }^{6} \mathrm{LiF}$ volume fraction is 1.0.

Similar results were obtained for an infinite slab system. This system was modeled with the 2-D SWAN in R-Z geometry having the neutron source at one of the cylinder bases and imposing reflective radial boundary conditions.

Additional tests are being conducted to verify the validity of the 2-D SWAN code to optimize the design of two-dimensional systems. Tests are also being performed to find how to apply the 2-D SWAN most efficiently, so as to minimize the running time without sacrificing accuracy.

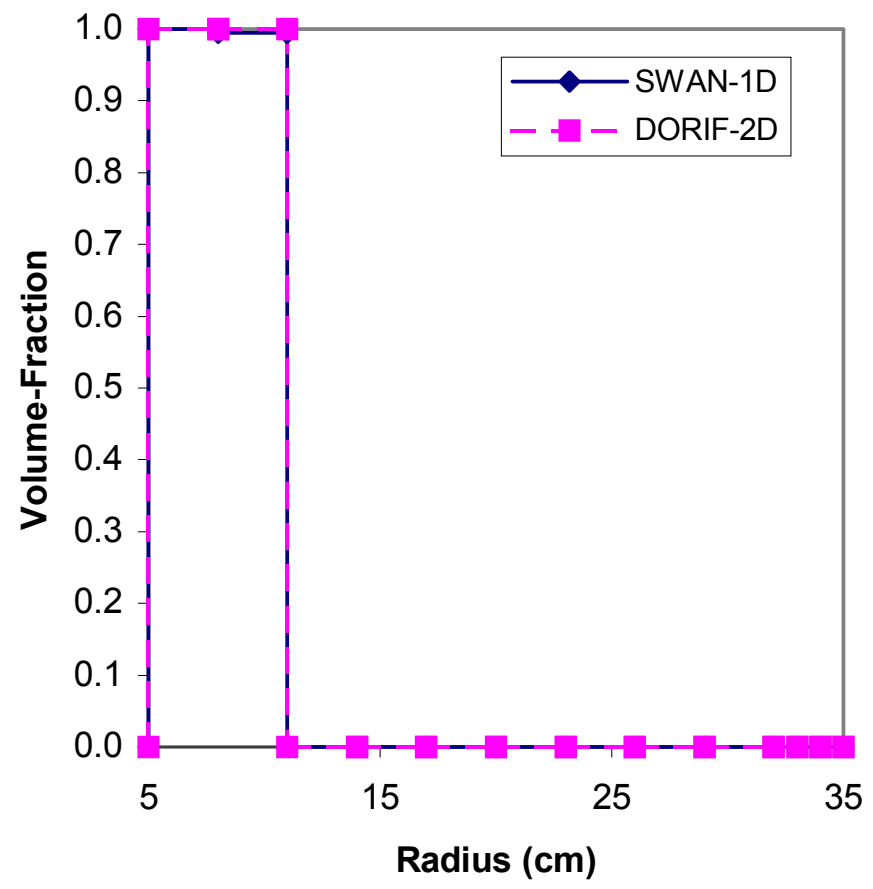


Figure 7.1 Comparison of optimal ${ }^{7} \mathrm{LiF}$ concentration

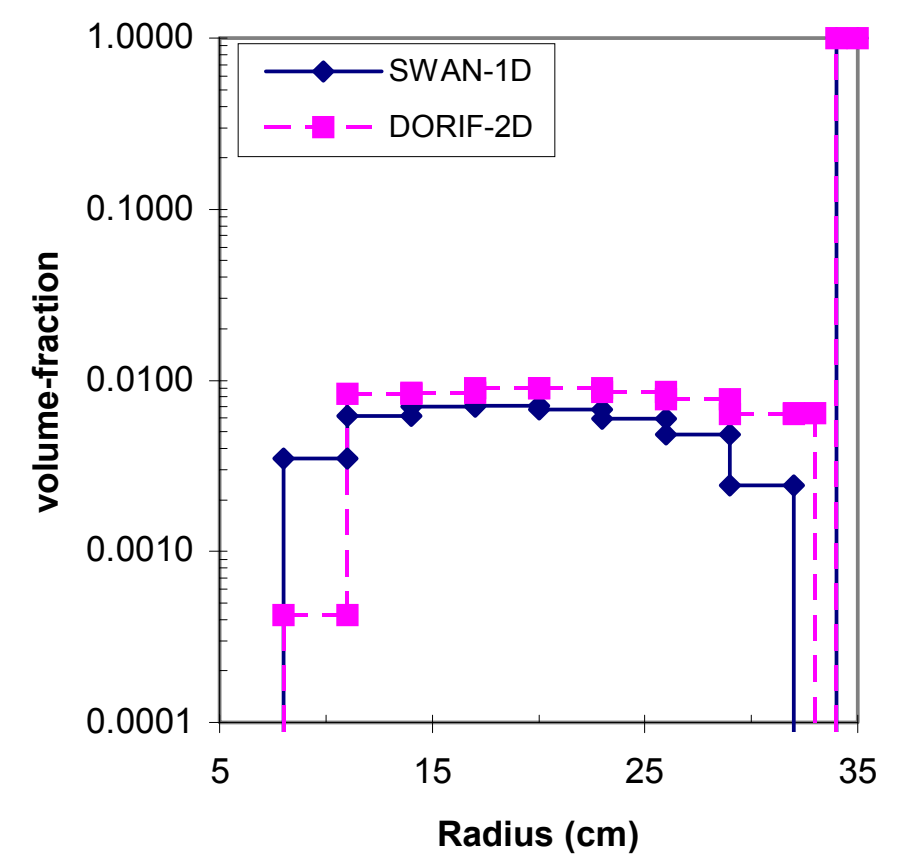

Figure 7.2 Comparison of optimal ${ }^{6} \mathrm{LiF}$ concentration

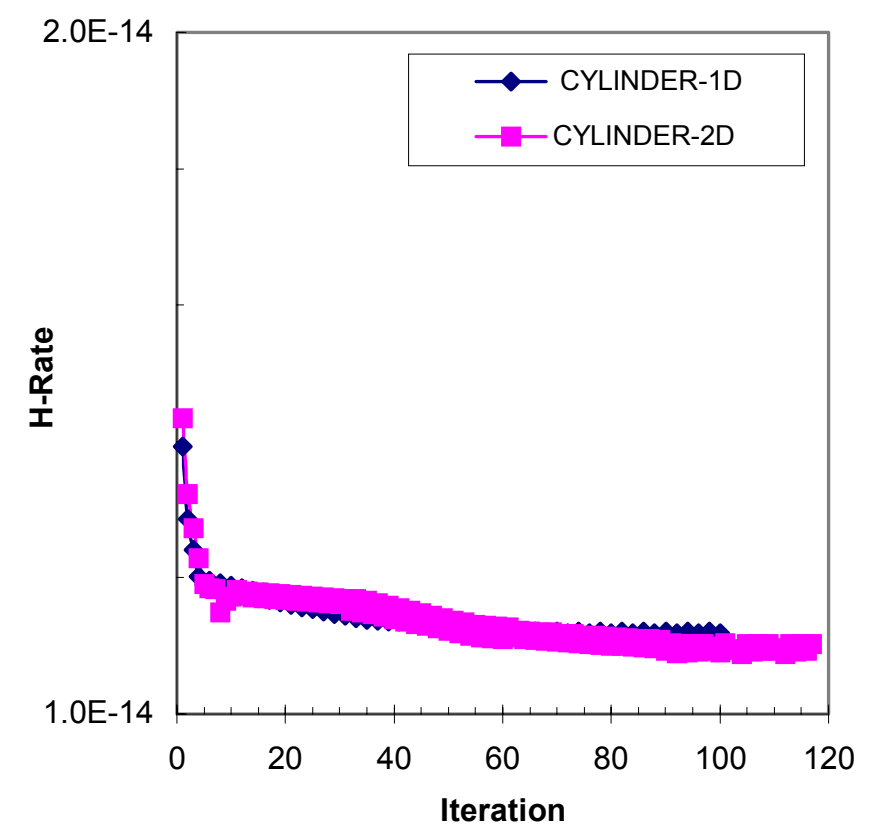

Figure 7.3 Comparison of evolution of dose-equivalent to tumor located between 8 to $9 \mathrm{~cm}$ inside brain 


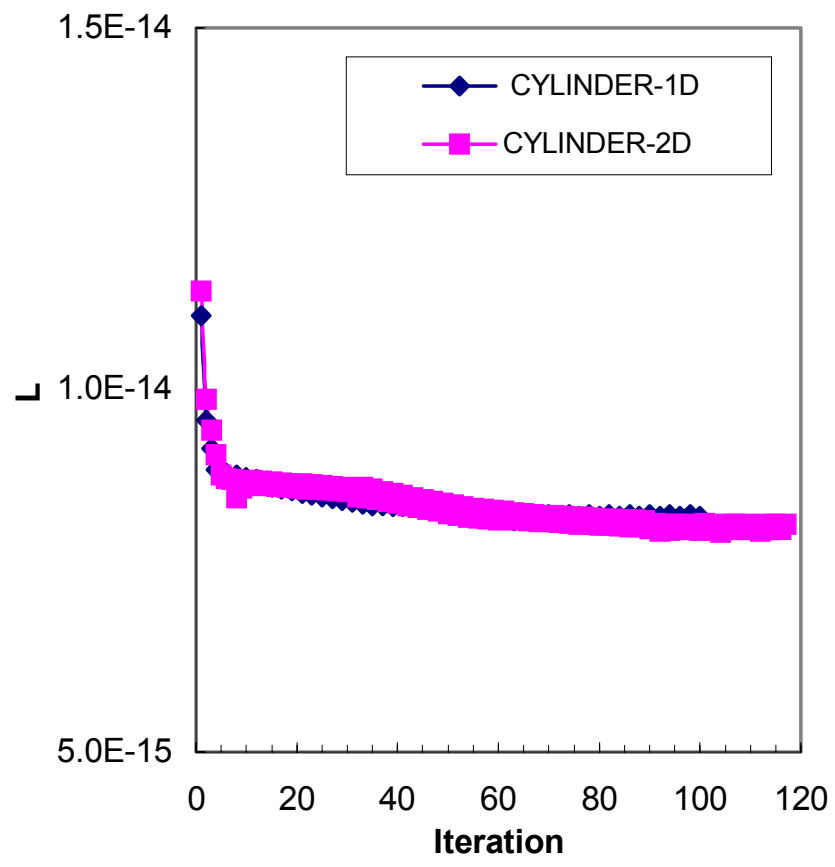

Figure 7.4 Comparison of evolution of dose-equivalent to normal tissue at outermost $1 \mathrm{~cm}$ of brain

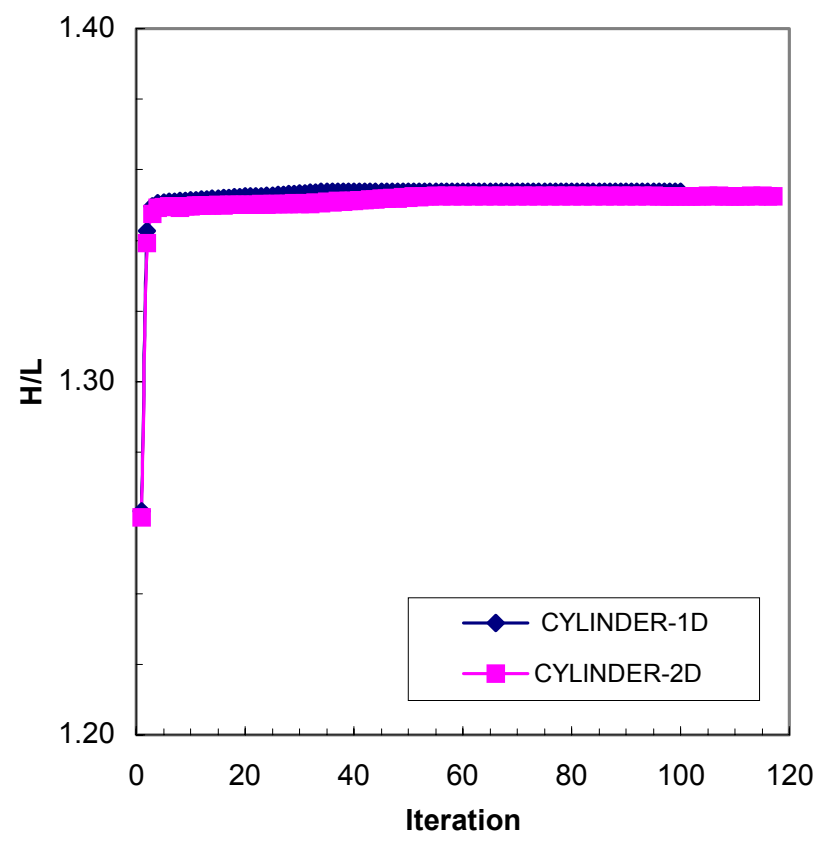


Figure 7.5 Comparison of $\mathrm{H} / \mathrm{L}$ ratio

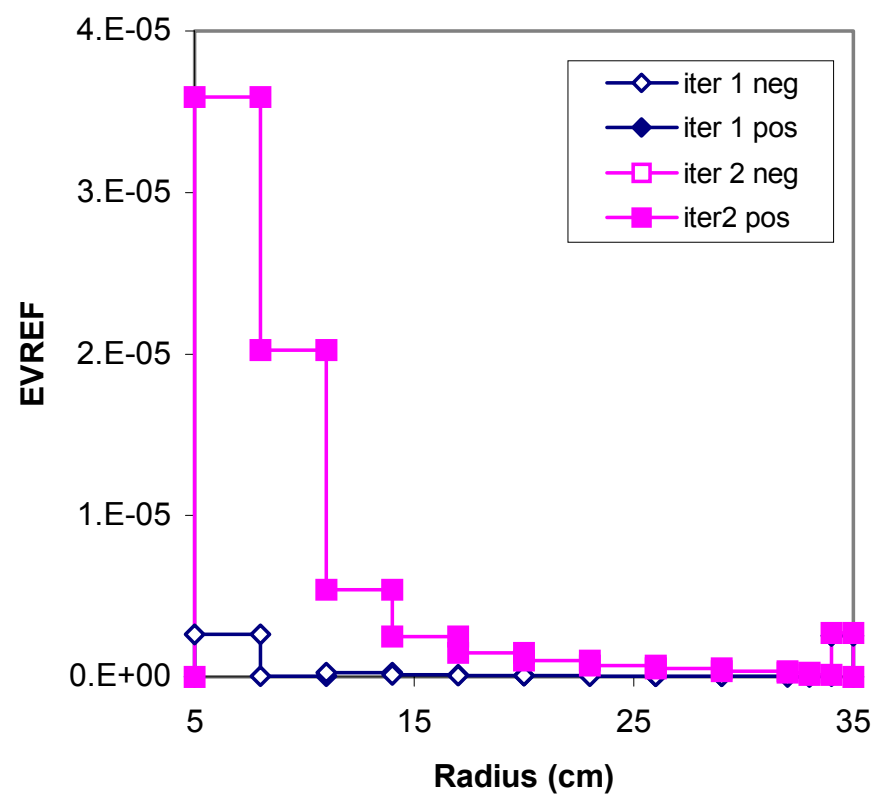

Figure 7.6 EVREF of ${ }^{6} \mathrm{LiF}$ Vs. $\mathrm{MgF}_{2}$ for successive iterations after the 2-D optimization has converged

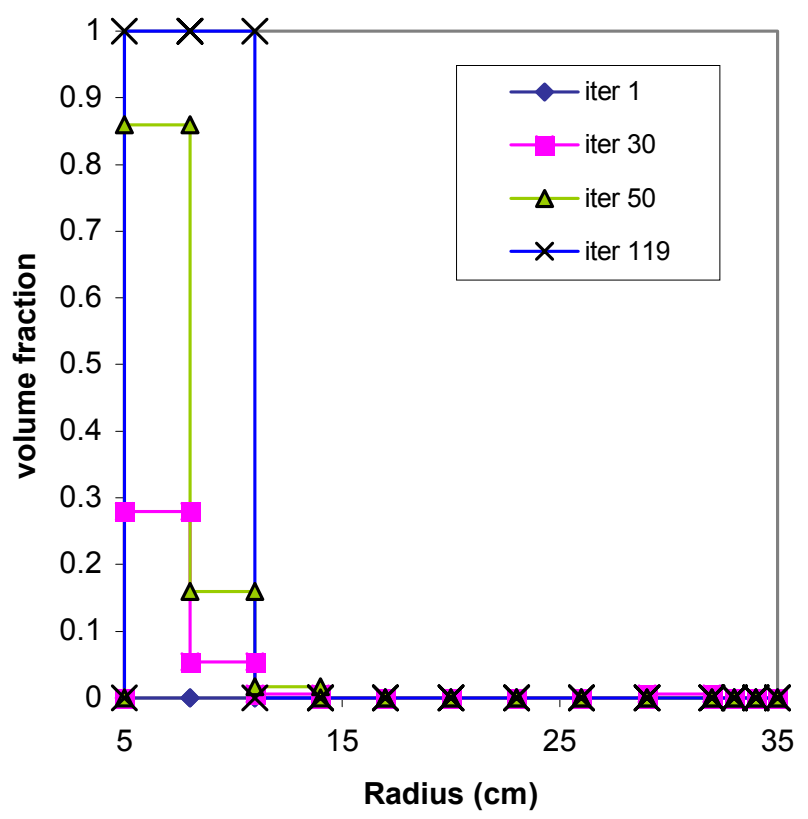


Figure $7.7^{7} \mathrm{LiF}$ concentration evolution in 2-D optimization

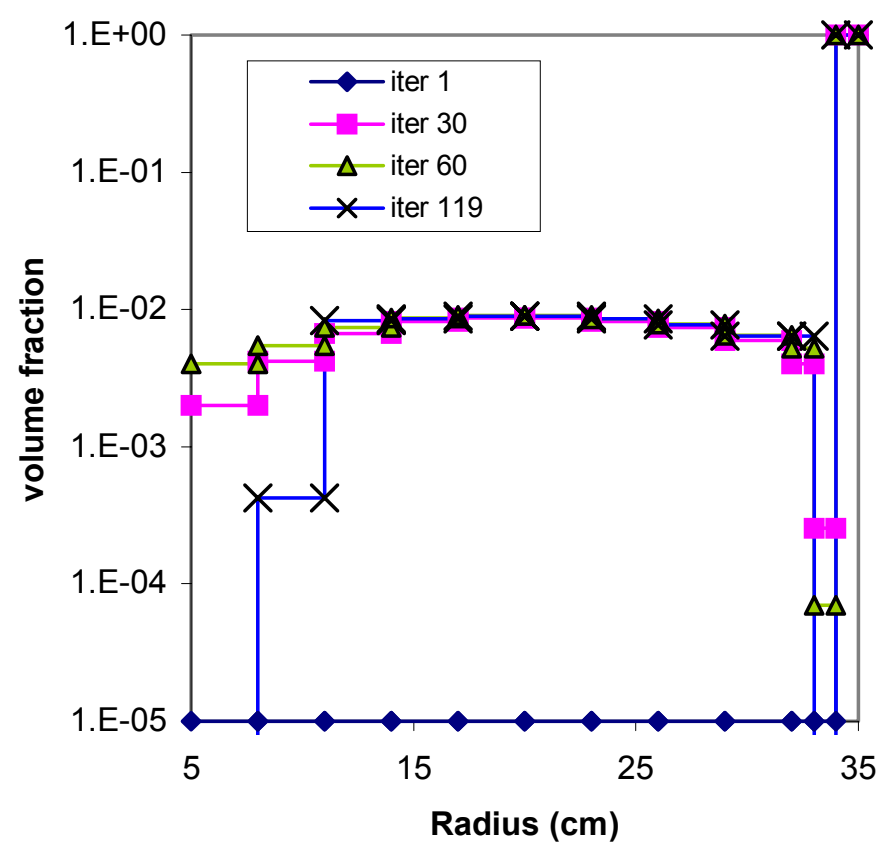

Figure $7.8{ }^{6} \mathrm{LiF}$ concentration evolution in 2-D optimization 


\section{DORT CONVERGENCE PROBLEMS}

\subsection{INTRODUCTION}

Although DORIF converged properly for the simple problems considered for the code validation testing, we encountered severe convergence problems for more realistic two-dimensional problems. Particularly problematic is the convergence of the adjoint solutions. In this section we'll illustrate the convergence problems we have encountered.

\subsection{TEST PROBLEM DESCRIPTION}

The geometry of the problem considered is schematically described in Figure 8.1. This is a cylindrical problem that is described in R-Z geometry. The outer dimensions are radius of 100 $\mathrm{cm}$ and height of $252 \mathrm{~cm}$. There is a source-vacuum tunnel through which protons from an accelerator (not shown) enter the system and go to the beryllium target that is located $119 \mathrm{~cm}$ inside the system. The evacuated beam tube is $5 \mathrm{~cm}$ in radius. Its lower $100 \mathrm{~cm}$ is surrounded by a $\mathrm{Pb}$ reflector up to a radius of $100 \mathrm{~cm}$. The remaining $20 \mathrm{~cm}$ in height is surrounded by a U/Zr shell having an outer radius of $18.2 \mathrm{~cm}$. From this radius up to a radius of $100 \mathrm{~cm}$ there is a $\mathrm{Pb}$ reflector at $80 \%$ of its theoretical density. Above the source vacuum channel there is a ${ }^{9} \mathrm{Be}$ target disc that is $2 \mathrm{~cm}$ in height and $5 \mathrm{~cm}$ in radius. This disc is surrounded by $\mathrm{U} / \mathrm{Zr}$ of $18.2 \mathrm{~cm}$ outer radius and then by $80 \%$ nominal density $\mathrm{Pb}$ reflector up to a radius of $100 \mathrm{~cm}$. Above this $\mathrm{Be}$ disc there is a $21 \mathrm{~cm}$ high U/Zr cylinder $18.2 \mathrm{~cm}$ in radius that is surrounded by a reflector of $80 \%$ nominal density $\mathrm{Pb}$ up to a radius of $100 \mathrm{~cm}$. The U/Zr occupies only $80 \%$ of the volume; the other $20 \%$ is assumed used for air-cooling.

The cylinder of radius $100 \mathrm{~cm}$ and height $80 \mathrm{~cm}$ that follows on top of the $\mathrm{U} / \mathrm{Zr}$ segment is the beam shaping assembly (BSA). The BSA was split at a radius of $20 \mathrm{~cm}$ to inner and outer zones. A collimator having an outer radius of $60 \mathrm{~cm}$ with a central aperture that is $10 \mathrm{~cm}$ in radius is separating the BSA from the brain. It is surrounded by a pure $\mathrm{Pb}$ annulus up to a radius of 100 $\mathrm{cm}$.

The brain is modeled as a $20 \mathrm{~cm}$ high cylinder of radius $10 \mathrm{~cm}$. The brain is surrounded by a shield that is $60 \mathrm{~cm}$ in outer radius. This shield is referred in Figure 1 as "collimator II". The adjoint source $\mathrm{H}$, representing the tumor, is modeled as a central disc that is $1 \mathrm{~cm}$ in height and 1 $\mathrm{cm}$ in radius. It is located between 8 and $9 \mathrm{~cm}$ from the brain bottom. The adjoint source $\mathrm{L}$ is modeled by a similar volume disc that is located between 0 to $1 \mathrm{~cm}$ from the brain bottom. It measures the maximum dose received by the skin during the treatment.

The neutron source is a combination of an external source that is multiplied by a small subcritical fission assembly (SA). The external source is a neutron emitted from $19 \mathrm{MeV}$ protons impinging on a ${ }^{9} \mathrm{Be}$ target. In order to avoid fission iterations, the SA fission neutron source was determined off-line, and is added to the external neutron source to make a single effective external neutron source. So that in the SA-BSA system a combined direct source of this neutron and the fission rate in the $\mathrm{U} / \mathrm{Zr}$ is entered. With this approximation no more fissions need to be followed, so that only one outer-iteration is needed. 


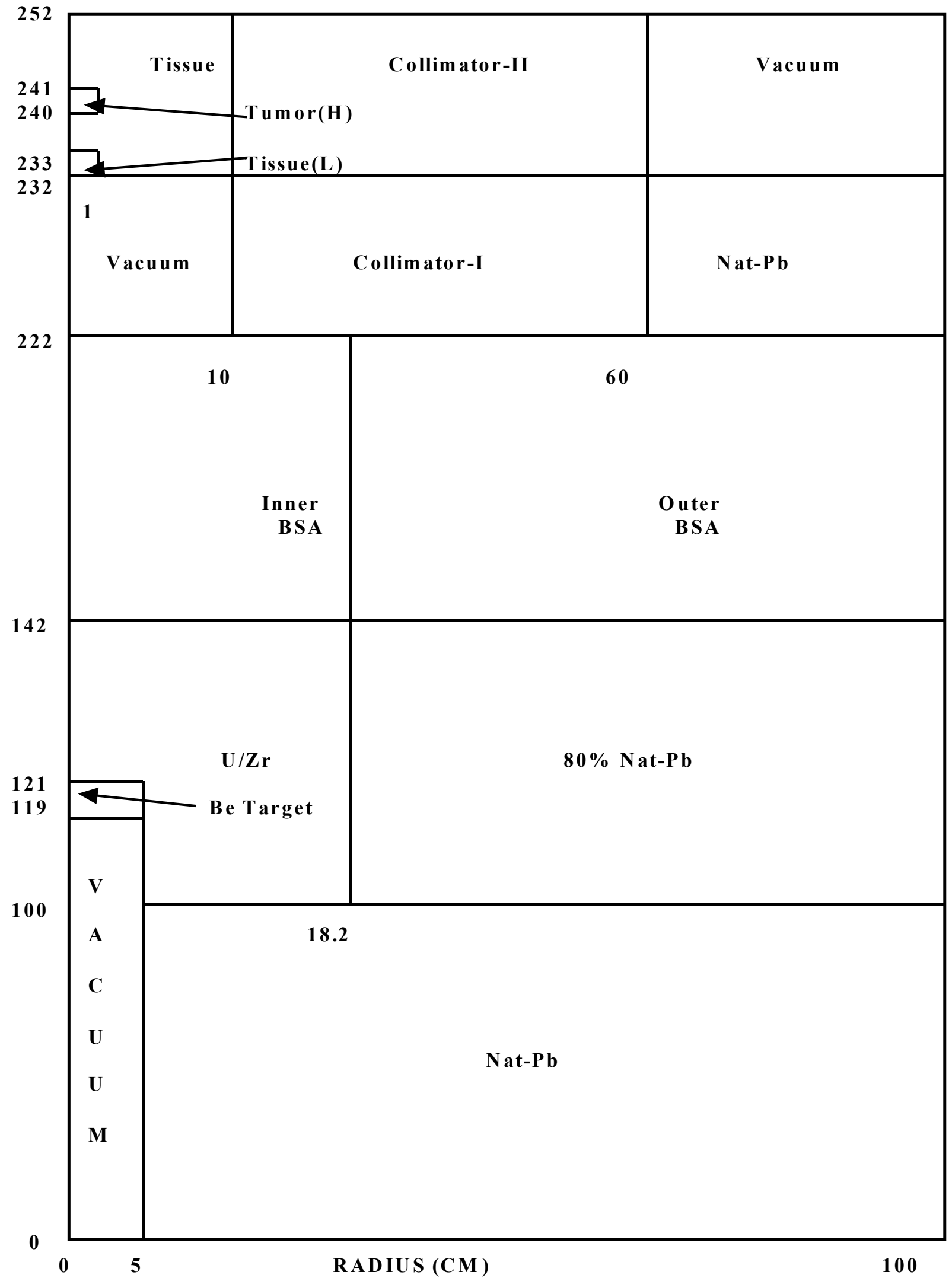

Figure 8.1 Geometry and composition of a two-dimensional test problem 
For the adjoint sources we take the proper energy-absorption XSECs divided by the detector volume and enlarged by factor of $10^{20}$. This multiplication is done in order to minimize distortion in the results due to round-off errors. The adjoint results are then scaled back by $10^{-20}$.

The BSA is composed of five materials: ${ }^{6} \mathrm{LiF}, \mathrm{Be}, \mathrm{Pb}, \mathrm{Fe}$ and $\mathrm{MgF}_{2}$. The spatial distribution of these constituents was assumed to be uniform with each constituent occupying $20 \%$ of the BSA volume. The inner and outer BSA have identical composition.

Collimator-I is assumed to have 75 volume \% Poly(H2O) of a uniform distribution plus a mixture of ${ }^{6} \mathrm{LiF}$ and $\mathrm{Pb}$. Collimator-II or the shield for the brain is taken to have a uniform mixture of 25 volume $\%{ }^{6} \mathrm{LiF}, 25 \% \mathrm{~Pb}$ and $50 \% \mathrm{Poly}\left(\mathrm{H}_{2} \mathrm{O}\right)$.

The system is divided into 305 zones and 100x252 RZ cells, $1 \mathrm{~cm}$ in height and $1 \mathrm{~cm}$ in radius. The $\mathrm{S}_{16}$ (160 angles)- $\mathrm{P}_{3}$ transport approximation is used for the DORT calculations. The maximum of iterations per energy group permitted is 200. The 69 group DABL cross section library is used for the calculations. It consists of 43 neutron groups and 23 photon groups. Table 8.1 gives the group energy boundaries.

Table 8.1 Energy Group Structure of the DABL Library

$\begin{array}{cl}\text { Group } & \text { Upper Energy(ev) } \\ \text { No. } & \text { NEUTRONS } \\ 1 & 1.9640 \mathrm{e}+7 \\ 2 & 1.6905 \\ 3 & 1.4918 \\ 4 & 1.4101 \\ 5 & 1.3840 \\ 6 & 1.2523 \\ 7 & 1.2214 \\ 8 & 1.1052 \\ 9 & 1.0000 \\ 10 & 9.0484 \mathrm{e}+6 \\ 11 & 8.1873 \\ 12 & 7.4082 \\ 13 & 6.3763 \\ 14 & 4.9659 \\ 15 & 4.7237 \\ 16 & 4.0657 \\ 17 & 3.0119 \\ 18 & 2.3852 \\ 19 & 2.3069 \\ 20 & 1.8268 \\ 21 & 1.4227 \\ 22 & 1.1080 \\ 23 & 9.6164 \mathrm{e}+5\end{array}$

\begin{tabular}{rlrl}
24 & 8.2085 & \multicolumn{3}{c}{ PHOTONS } \\
25 & 7.4274 & 47 & $2.0000 \mathrm{e}+7$ \\
26 & 6.3928 & 48 & 1.4000 \\
27 & 5.5023 & 49 & 1.2000 \\
28 & 3.6883 & 50 & 1.0000 \\
29 & 2.4724 & 51 & $8.0000 \mathrm{e}+6$ \\
30 & 1.5764 & 52 & 7.0000 \\
31 & 1.1109 & 53 & 6.0000 \\
32 & $5.2475 \mathrm{e}+4$ & 54 & 5.0000 \\
33 & 3.4307 & 55 & 4.0000 \\
34 & 2.4788 & 56 & 3.0000 \\
35 & 2.1875 & 57 & 2.5000 \\
36 & 1.0333 & 58 & 2.0000 \\
37 & $3.3546 \mathrm{e}+3$ & 59 & 1.5000 \\
38 & 1.2341 & 60 & 1.0000 \\
39 & $5.8295 \mathrm{e}+2$ & 61 & $7.0000 \mathrm{e}+5$ \\
40 & 2.7536 & 62 & 4.5000 \\
41 & 1.0130 & 63 & 3.0000 \\
42 & $2.9023 \mathrm{e}+1$ & 64 & 1.5000 \\
43 & 1.0677 & 65 & 1.0000 \\
44 & $3.0590 \mathrm{e}+0$ & 66 & $7.0000 \mathrm{e}+4$ \\
45 & 1.1253 & 67 & 4.5000 \\
46 & $4.1399 \mathrm{e}-1$ & 68 & 3.0000 \\
lower energy $1.0000 \mathrm{e}-5$ & 69 & 2.0000 \\
& & $10 w e r$ & energy \\
& & & \\
\end{tabular}




\subsection{CONVERGENCE PROBLEMS}

Shown in Tables 8.2, 8.3 and 8.4 are the convergence tables edited by DORT for the above sample problem. Table 8.2 is for the forward (flux) solution whereas Tables 8.3 and 8.4 are for the adjoint solutions; Table 8.3 for the adjoint corresponding to the tumor (" $\mathrm{H}$ " adjoint) and Table 8.4 for the adjoint corresponding to the skin ("L" adjoint). The leftmost column is the energy group number. The second column from left is the number of inner iterations performed by DORT in the specific group; the limit set of this number is 200 . The fourth column from left is the maximum deviation of the value of the flux from the previous iteration; the convergence limit specified for this problem is $10^{-4}$.

Table 8.2 Convergence Data for DORT Sample Problem - Forward Solution

\begin{tabular}{rcccccc} 
grp itn imfd jmfd mx fx dv & \multicolumn{1}{l}{$m x$ dv } \\
1 & 200 & 24 & 33 & $-6.010 \mathrm{E}-04$ & $6.850 \mathrm{E}-12$ \\
2 & 200 & 74 & 133 & $2.260 \mathrm{E}-04$ & $1.020 \mathrm{E}-10$ \\
3 & 200 & 58 & 170 & $-5.680 \mathrm{E}-04$ & $8.740 \mathrm{E}-11$ \\
4 & 200 & 1 & 224 & $9.590 \mathrm{E}-04$ & $1.110 \mathrm{E}-12$ \\
5 & 200 & 10 & 13 & $-5.260 \mathrm{E}-04$ & $6.310 \mathrm{E}-09$ \\
6 & 200 & 90 & 233 & $-2.280 \mathrm{E}-05$ & $3.980 \mathrm{E}-15$ \\
7 & 200 & 100 & 245 & $-2.160 \mathrm{E}-05$ & $2.700 \mathrm{E}-14$ \\
8 & 200 & 100 & 243 & $2.270 \mathrm{E}-05$ & $3.520 \mathrm{E}-14$ \\
9 & 123 & 97 & 251 & $9.720 \mathrm{E}-06$ & $6.140 \mathrm{E}-14$ \\
10 & 200 & 62 & 13 & $-4.600 \mathrm{E}-04$ & $8.820 \mathrm{E}-12$ \\
11 & 70 & 99 & 248 & $9.440 \mathrm{E}-06$ & $7.320 \mathrm{E}-14$ \\
12 & 52 & 96 & 252 & $9.710 \mathrm{E}-06$ & $1.770 \mathrm{E}-13$ \\
13 & 200 & 100 & 244 & $2.930 \mathrm{E}-05$ & $1.680 \mathrm{E}-13$ \\
14 & 200 & 97 & 251 & $3.240 \mathrm{E}-05$ & $5.160 \mathrm{E}-14$ \\
15 & 200 & 100 & 235 & $8.550 \mathrm{E}-05$ & $5.830 \mathrm{E}-14$ \\
16 & 200 & 99 & 248 & $-1.190 \mathrm{E}-04$ & $2.790 \mathrm{E}-13$ \\
17 & 200 & 100 & 246 & $-1.440 \mathrm{E}-04$ & $2.930 \mathrm{E}-13$ \\
18 & 200 & 100 & 244 & $-1.300 \mathrm{E}-04$ & $5.600 \mathrm{E}-14$ \\
19 & 200 & 100 & 247 & $2.010 \mathrm{E}-04$ & $8.530 \mathrm{E}-13$ \\
20 & 200 & 99 & 248 & $1.610 \mathrm{E}-04$ & $1.870 \mathrm{E}-12$ \\
21 & 200 & 100 & 245 & $-1.200 \mathrm{E}-04$ & $2.280 \mathrm{E}-12$ \\
22 & 200 & 100 & 246 & $-2.690 \mathrm{E}-04$ & $1.800 \mathrm{E}-12$ \\
23 & 200 & 100 & 244 & $-1.580 \mathrm{E}-04$ & $1.990 \mathrm{E}-12$ \\
24 & 200 & 98 & 252 & $-1.490 \mathrm{E}-04$ & $1.510 \mathrm{E}-12$ \\
25 & 200 & 98 & 251 & $2.260 \mathrm{E}-04$ & $2.750 \mathrm{E}-12$ \\
26 & 200 & 100 & 243 & $-2.100 \mathrm{E}-04$ & $2.140 \mathrm{E}-12$ \\
27 & 200 & 100 & 244 & $-1.750 \mathrm{E}-04$ & $4.770 \mathrm{E}-12$ \\
28 & 200 & 100 & 245 & $1.620 \mathrm{E}-04$ & $2.510 \mathrm{E}-12$ \\
29 & 200 & 100 & 247 & $-2.590 \mathrm{E}-04$ & $2.730 \mathrm{E}-12$ \\
30 & 200 & 99 & 249 & $-2.270 \mathrm{E}-04$ & $2.330 \mathrm{E}-12$ \\
31 & 200 & 100 & 243 & $3.240 \mathrm{E}-04$ & $5.060 \mathrm{E}-12$ \\
32 & 200 & 100 & 243 & $-2.030 \mathrm{E}-04$ & $2.920 \mathrm{E}-12$ \\
33 & 200 & 100 & 245 & $-2.750 \mathrm{E}-04$ & $1.770 \mathrm{E}-12$ \\
34 & 200 & 100 & 246 & $-3.520 \mathrm{E}-04$ & $1.200 \mathrm{E}-12$
\end{tabular}

$\begin{array}{lllllll}35 & 200 & 100 & 248 & -1.080 \mathrm{E}-04 & 9.560 \mathrm{E}-12 \\ 36 & 200 & 100 & 243 & -2.500 \mathrm{E}-04 & 1.010 \mathrm{E}-11 \\ 37 & 200 & 63 & 251 & 1.490 \mathrm{E}-04 & 1.920 \mathrm{E}-11 \\ 38 & 200 & 100 & 244 & -2.420 \mathrm{E}-04 & 3.500 \mathrm{E}-12 \\ 39 & 200 & 100 & 244 & -3.610 \mathrm{E}-04 & 2.080 \mathrm{E}-12 \\ 40 & 200 & 100 & 233 & -3.900 \mathrm{E}-04 & 6.220 \mathrm{E}-13 \\ 41 & 200 & 100 & 233 & 6.840 \mathrm{E}-04 & 3.800 \mathrm{E}-13 \\ 42 & 200 & 100 & 235 & -1.230 \mathrm{E}-04 & 2.370 \mathrm{E}-13 \\ 43 & 200 & 100 & 234 & 3.160 \mathrm{E}-04 & 1.220 \mathrm{E}-13 \\ 44 & 200 & 99 & 232 & 9.480 \mathrm{E}-05 & 2.180 \mathrm{E}-14 \\ 45 & 200 & 100 & 233 & 2.460 \mathrm{E}-04 & 1.820 \mathrm{E}-14 \\ 46 & 200 & 46 & 216 & -4.030 \mathrm{E}-05 & 1.610 \mathrm{E}-18 \\ 47 & 200 & 18 & 63 & 2.940 \mathrm{E}+04 & 2.300 \mathrm{E}-20 \\ 48 & 200 & 54 & 10 & -1.530 \mathrm{E}+04 & 6.130 \mathrm{E}-28 \\ 49 & 200 & 100 & 233 & 8.720 \mathrm{E}-05 & 8.700 \mathrm{E}-17 \\ 50 & 200 & 100 & 234 & 1.470 \mathrm{E}-04 & 3.540 \mathrm{E}-16 \\ 51 & 126 & 96 & 251 & -8.340 \mathrm{E}-06 & 2.150 \mathrm{E}-13 \\ 52 & 200 & 100 & 244 & 6.650 \mathrm{E}-05 & 1.530 \mathrm{E}-14 \\ 53 & 200 & 100 & 244 & 9.560 \mathrm{E}-05 & 1.370 \mathrm{E}-14 \\ 54 & 128 & 88 & 233 & 7.390 \mathrm{E}-06 & 2.700 \mathrm{E}-14 \\ 55 & 68 & 88 & 233 & 9.470 \mathrm{E}-06 & 3.180 \mathrm{E}-14 \\ 56 & 200 & 100 & 246 & 2.020 \mathrm{E}-04 & 6.230 \mathrm{E}-14 \\ 57 & 145 & 100 & 233 & -7.400 \mathrm{E}-06 & 7.670 \mathrm{E}-15 \\ 58 & 200 & 2 & 252 & -4.680 \mathrm{E}-05 & 2.940 \mathrm{E}-11 \\ 59 & 47 & 100 & 244 & 8.980 \mathrm{E}-06 & 5.250 \mathrm{E}-13 \\ 60 & 200 & 90 & 233 & -1.000 \mathrm{E}-04 & 3.200 \mathrm{E}-13 \\ 61 & 200 & 82 & 233 & -1.520 \mathrm{E}-04 & 8.210 \mathrm{E}-13 \\ 62 & 200 & 64 & 251 & -2.790 \mathrm{E}-04 & 2.830 \mathrm{E}-13 \\ 63 & 200 & 67 & 248 & 6.420 \mathrm{E}-04 & 1.240 \mathrm{E}-13 \\ 64 & 200 & 65 & 251 & 9.680 \mathrm{E}-04 & 1.220 \mathrm{E}-14 \\ 65 & 200 & 100 & 235 & -6.130 \mathrm{E}-04 & 4.000 \mathrm{E}-17 \\ 66 & 200 & 69 & 248 & 3.290 \mathrm{E}-04 & 2.110 \mathrm{E}-18 \\ 67 & 200 & 7 & 92 & -6.250 \mathrm{E}-04 & 1.070 \mathrm{E}-13 \\ 68 & 200 & 20 & 119 & 1.350 \mathrm{E}-03 & 1.000 \mathrm{E}-16 \\ 69 & 200 & 21 & 109 & -2.440 \mathrm{E}-02 & 1.610 \mathrm{E}-21\end{array}$

It is seen that the direct DORT calculations did not converge well enough in many of the energy groups - the convergence criterion was not reached even after 200 iterations. The situation is much worse in the adjoint calculations (Tables 8.3 and 8.4). In fact, large fluctuations in the 
results were observed between consequitive iterations in these calculations. Eventually the calculations "crashed". The evolution towards such "crashing is illustrated in Table 8.5; it pertains to group 13 of the " $\mathrm{H}$ " adjoint.

Table 8.3 Convergence Data for DORT Sample Problem - Adjoint "H" Solution

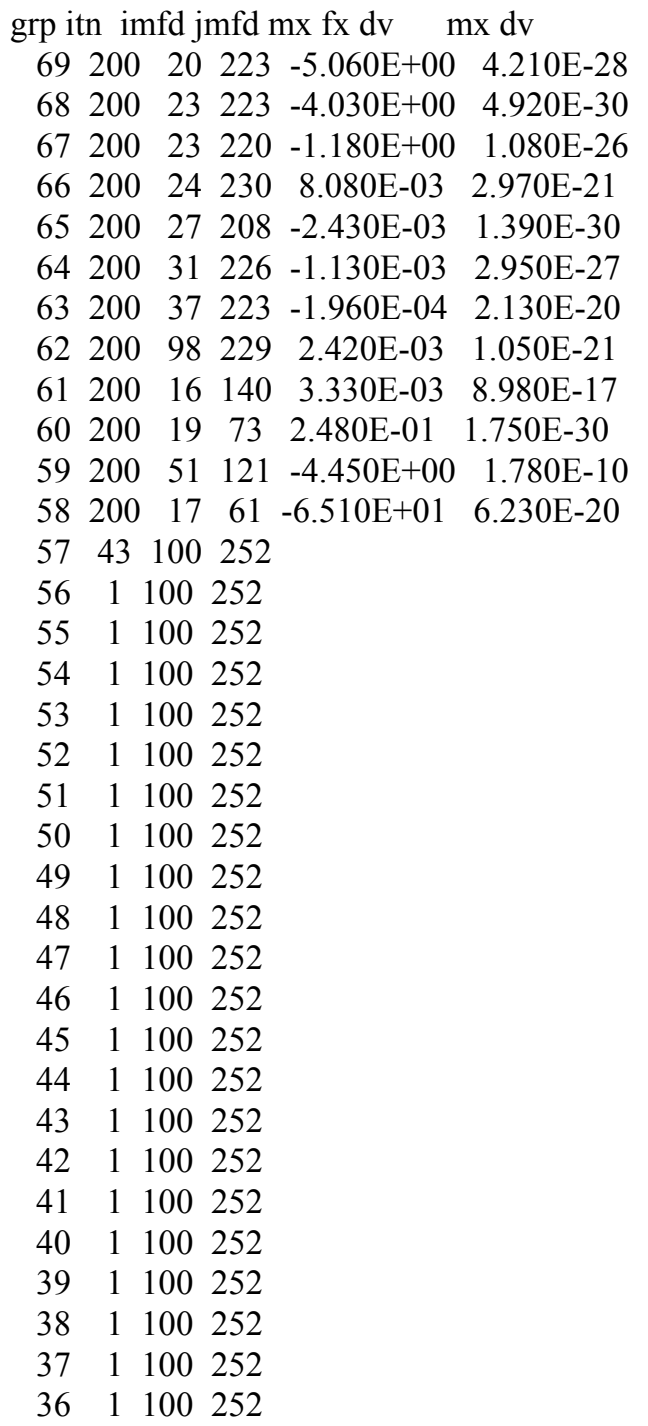

$\begin{array}{cccc}35 & 1 & 100 & 252 \\ 34 & 1 & 100 & 252 \\ 33 & 1 & 100 & 252 \\ 32 & 1 & 100 & 252 \\ 31 & 1 & 100 & 252 \\ 30 & 1 & 100 & 252 \\ 29 & 1 & 100 & 252 \\ 28 & 1 & 100 & 252 \\ 27 & 1 & 100 & 252 \\ 26 & 1 & 100 & 252 \\ 25 & 1 & 100 & 252 \\ 24 & 1 & 100 & 252 \\ 23 & 1 & 100 & 252 \\ 22 & 1 & 100 & 252 \\ 21 & 1 & 100 & 252 \\ 20 & 1 & 100 & 252 \\ 19 & 1 & 100 & 252 \\ 18 & 1 & 100 & 252 \\ 17 & 1 & 100 & 252 \\ 16 & 1 & 100 & 252 \\ 15 & 1 & 100 & 252 \\ 14 & 1 & 100 & 252 \\ 13 & 1 & 100 & 252 \\ 12 & 1 & 100 & 252 \\ 11 & 1 & 100 & 252 \\ 10 & 1 & 100 & 252 \\ 9 & 1 & 100 & 252 \\ 8 & 1 & 100 & 252 \\ 7 & 1 & 100 & 252 \\ 6 & 1 & 100 & 252 \\ 5 & 1 & 100 & 252 \\ 4 & 1 & 100 & 252 \\ 3 & 1 & 100 & 252 \\ 2 & 1 & 100 & 252 \\ 1 & 1 & 100 & 252\end{array}$

Table 8.4 Convergence Data for DORT Sample Problem - Adjoint "L" Solution

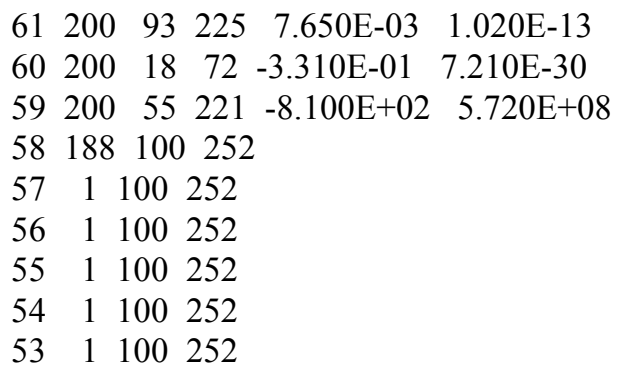




$\begin{array}{llll}52 & 1 & 100 & 252 \\ 51 & 1 & 100 & 252 \\ 50 & 1 & 100 & 252 \\ 49 & 1 & 100 & 252 \\ 48 & 1 & 100 & 252 \\ 47 & 1 & 100 & 252 \\ 46 & 1 & 100 & 252 \\ 45 & 1 & 100 & 252 \\ 44 & 1 & 100 & 252 \\ 43 & 1 & 100 & 252 \\ 42 & 1 & 100 & 252 \\ 41 & 1 & 100 & 252 \\ 40 & 1 & 100 & 252 \\ 39 & 1 & 100 & 252 \\ 38 & 1 & 100 & 252 \\ 37 & 1 & 100 & 252 \\ 36 & 1 & 100 & 252 \\ 35 & 1 & 100 & 252 \\ 34 & 1 & 100 & 252 \\ 33 & 1 & 100 & 252 \\ 32 & 1 & 100 & 252 \\ 31 & 1 & 100 & 252 \\ 30 & 1 & 100 & 252 \\ 29 & 1 & 100 & 252 \\ 28 & 1 & 100 & 252 \\ 27 & 1 & 100 & 252\end{array}$

$\begin{array}{cccc}26 & 1 & 100 & 252 \\ 25 & 1 & 100 & 252 \\ 24 & 1 & 100 & 252 \\ 23 & 1 & 100 & 252 \\ 22 & 1 & 100 & 252 \\ 21 & 1 & 100 & 252 \\ 20 & 1 & 100 & 252 \\ 19 & 1 & 100 & 252 \\ 18 & 1 & 100 & 252 \\ 17 & 1 & 100 & 252 \\ 16 & 1 & 100 & 252 \\ 15 & 1 & 100 & 252 \\ 14 & 1 & 100 & 252 \\ 13 & 1 & 100 & 252 \\ 12 & 1 & 100 & 252 \\ 11 & 1 & 100 & 252 \\ 10 & 1 & 100 & 252 \\ 9 & 1 & 100 & 252 \\ 8 & 1 & 100 & 252 \\ 7 & 1 & 100 & 252 \\ 6 & 1 & 100 & 252 \\ 5 & 1 & 100 & 252 \\ 4 & 1 & 100 & 252 \\ 3 & 1 & 100 & 252 \\ 2 & 1 & 100 & 252 \\ 1 & 1 & 100 & 252\end{array}$

Table 8.5 Convergence Data for DORT Sample Problem - Adjoint "H” Solution Group 13 Iterations

\begin{tabular}{rccccc}
\multicolumn{6}{c}{ grp itn imfd jmfd*mx fx dv*mx dv fx } \\
13 & 1 & 100 & $118-2.26 \mathrm{E}+01$ & $4.24 \mathrm{E}-08$ \\
13 & 2 & 23 & $57-1.92 \mathrm{E}+00$ & $7.18 \mathrm{E}-20$ \\
13 & 3 & 23 & $68-2.84 \mathrm{E}+02$ & $3.98 \mathrm{E}-20$ \\
13 & 4 & 25 & $67-2.46 \mathrm{E}+02$ & $2.00 \mathrm{E}-20$ \\
13 & 5 & 18 & 49 & $4.40 \mathrm{E}+02$ & $6.39 \mathrm{E}-20$ \\
13 & 6 & 25 & 15 & $2.20 \mathrm{E}+03$ & $1.41 \mathrm{E}-23$ \\
13 & 7 & 37 & $10-2.60 \mathrm{E}+03$ & $8.17 \mathrm{E}-23$ \\
13 & 8 & 16 & 69 & $1.21 \mathrm{E}+03$ & $3.89 \mathrm{E}-17$ \\
13 & 9 & 16 & $241-6.00 \mathrm{E}+02$ & $1.10 \mathrm{E}+05$ \\
13 & 10 & 21 & $28-5.62 \mathrm{E}+02$ & $5.30 \mathrm{E}-18$ \\
13 & 11 & 16 & $37-4.05 \mathrm{E}+02$ & $4.39 \mathrm{E}-13$ \\
13 & 12 & 3 & 201 & $1.80 \mathrm{E}+03$ & $1.22 \mathrm{E}+00$ \\
13 & 13 & 19 & 55 & $3.68 \mathrm{E}+03$ & $3.84 \mathrm{E}-16$ \\
13 & 14 & 3 & $67-9.34 \mathrm{E}+03$ & $1.14 \mathrm{E}-09$ \\
13 & 15 & 22 & $242-1.74 \mathrm{E}+03$ & $1.34 \mathrm{E}+05$ \\
13 & 16 & 58 & 47 & $7.72 \mathrm{E}+03$ & $3.17 \mathrm{E}-23$ \\
13 & 17 & 32 & 54 & $7.09 \mathrm{E}+03$ & $1.53 \mathrm{E}-17$ \\
13 & 18 & 79 & 16 & $2.79 \mathrm{E}+03$ & $2.19 \mathrm{E}-28$ \\
13 & 19 & 12 & 143 & $9.16 \mathrm{E}+03$ & $2.28 \mathrm{E}-06$ \\
13 & 20 & 18 & 158 & $1.34 \mathrm{E}+03$ & $1.04 \mathrm{E}-01$ \\
13 & 21 & 36 & $232-3.66 \mathrm{E}+03$ & $6.84 \mathrm{E}+00$ \\
13 & 22 & 19 & 179 & $4.18 \mathrm{E}+03$ & $4.21 \mathrm{E}+01$
\end{tabular}

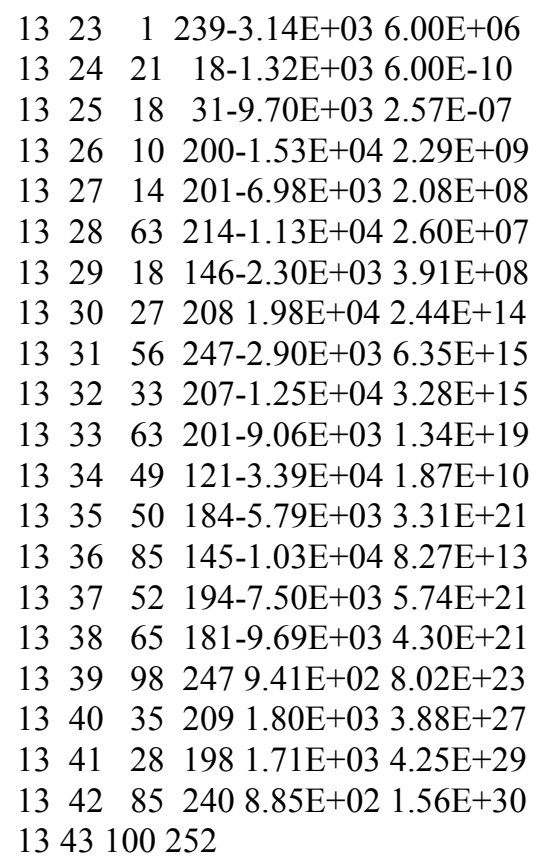


A significant better convergence is obtained with TWODANT, as illustrated in Tables 8.6 to 8.8. All three runs were converged in less than $\sim 20$ iterations, although in some of the groups there was a need for a negative flux fixup.

Table 8.6 Convergence Data for TWODANT Sample Problem - Forward Solution

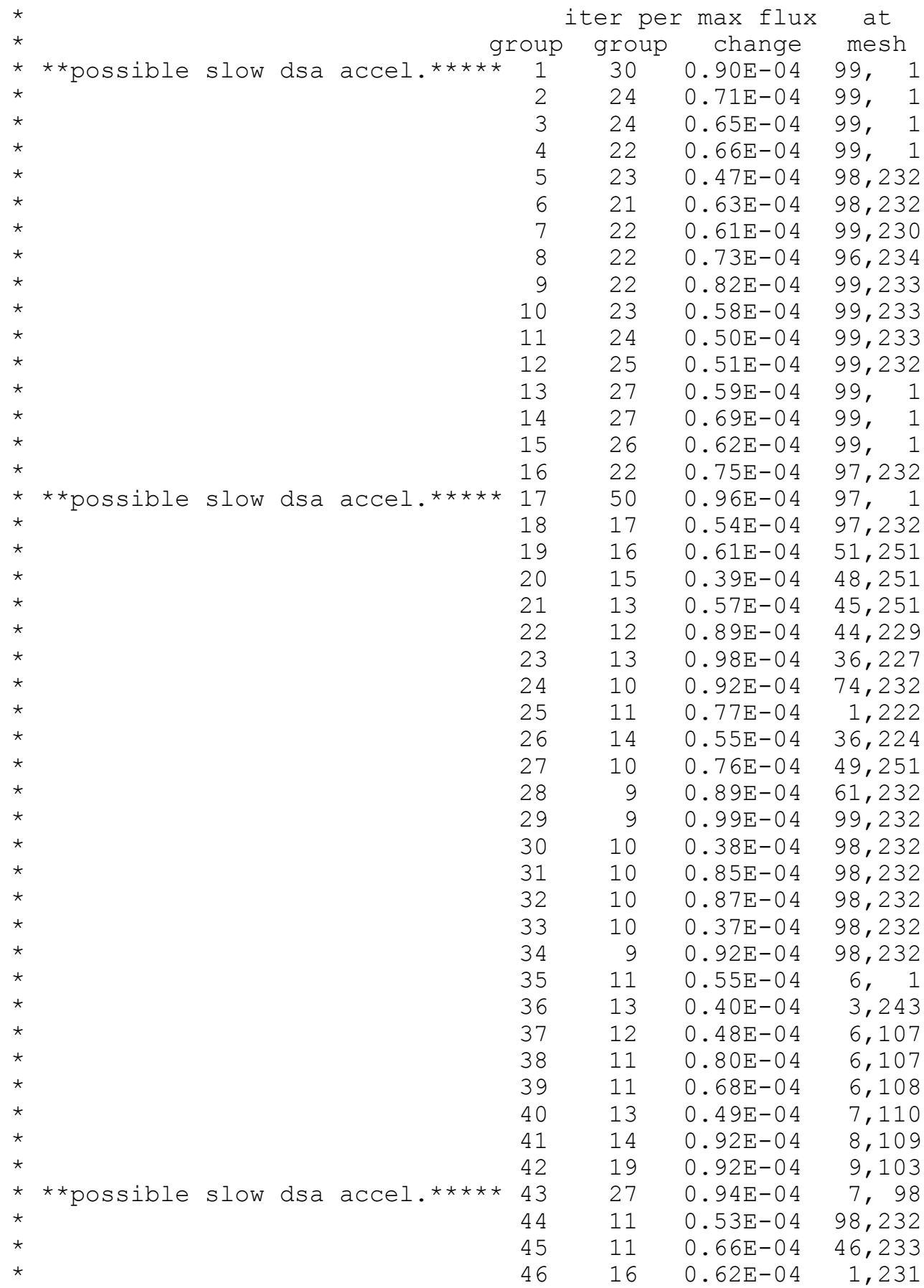




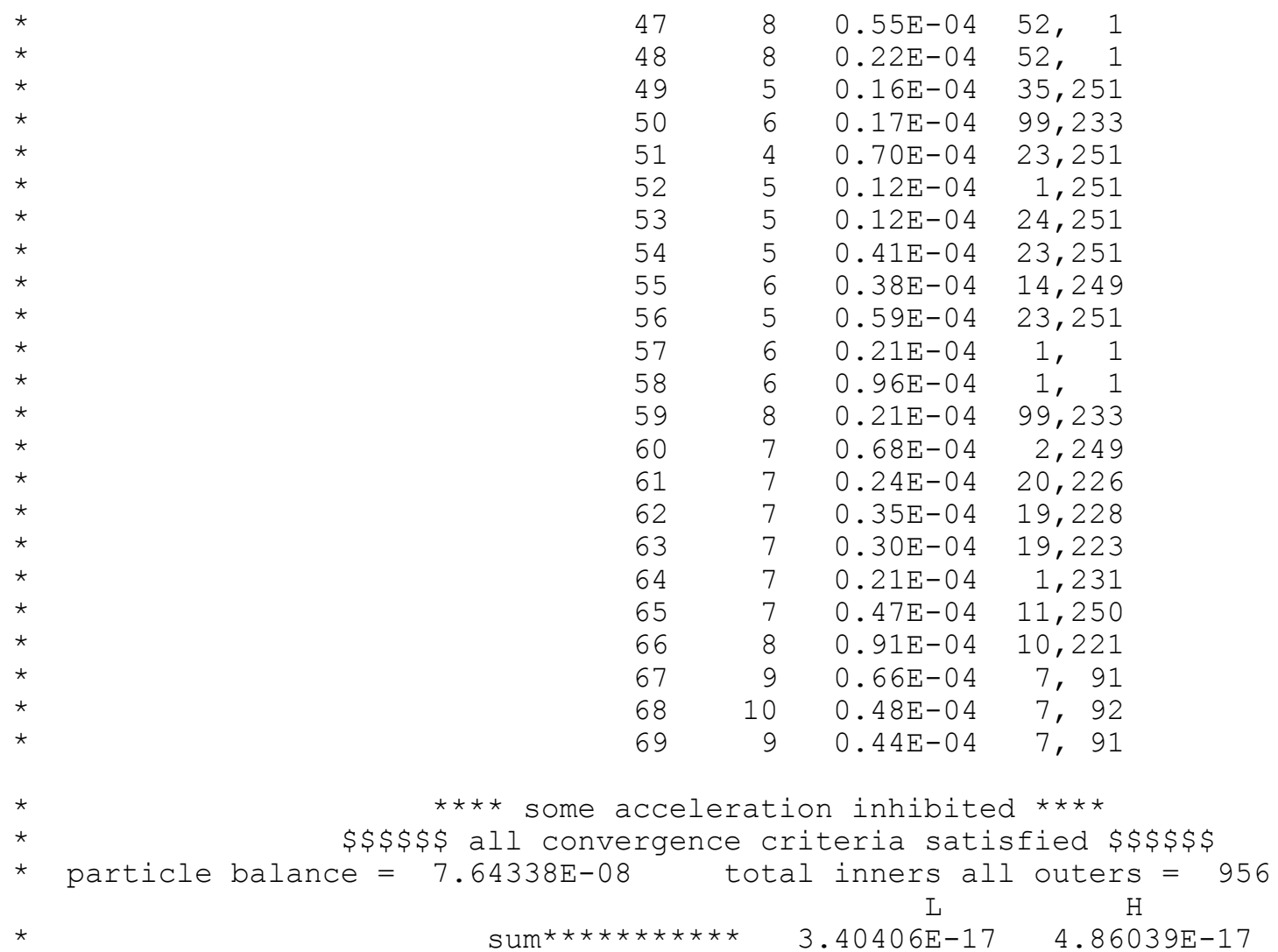

Table 8.7 Convergence Data for TWODANT Sample Problem - Adjoint "H" Solution

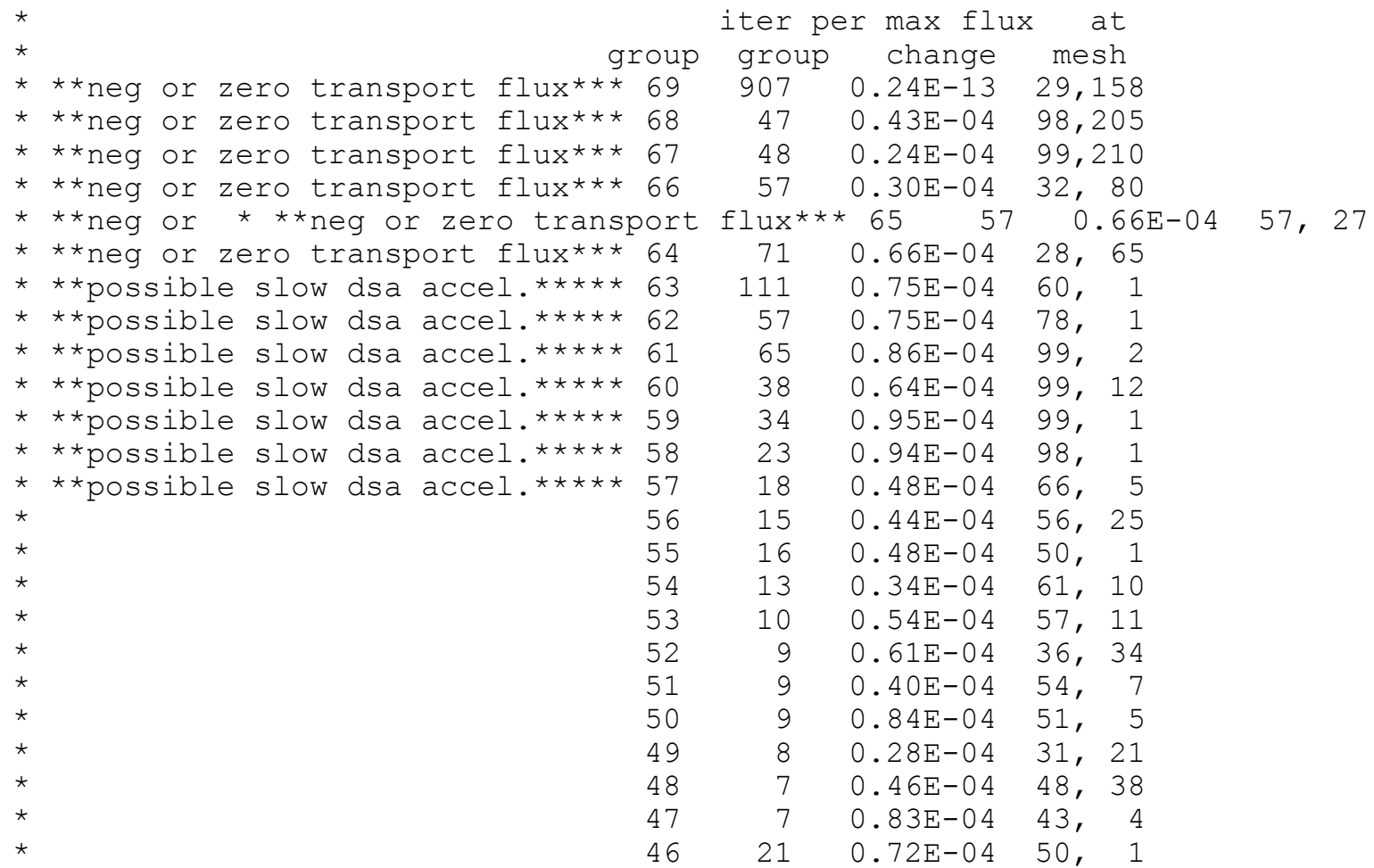




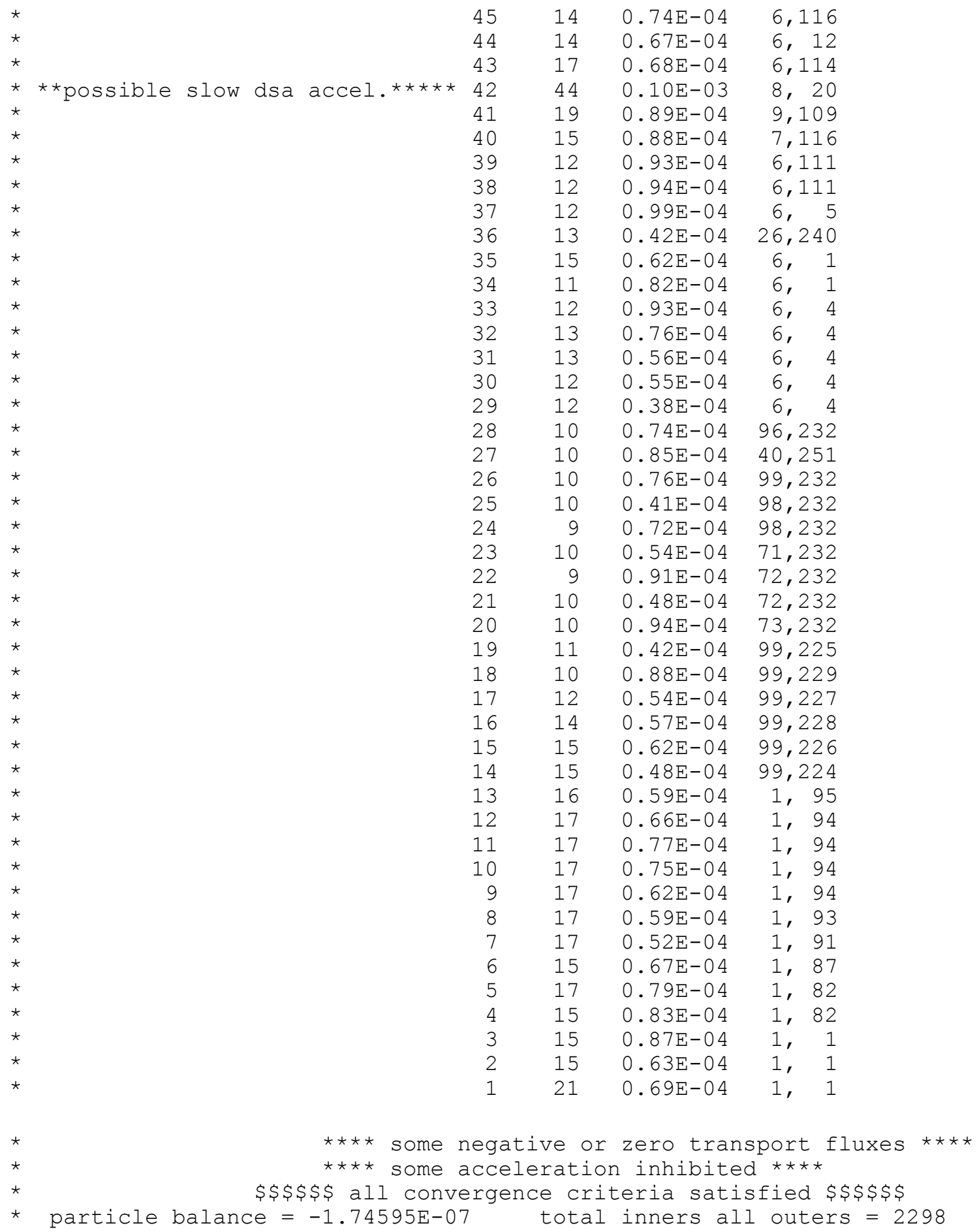

Table 8.8 Convergence Data for DORT Sample Problem - Adjoint "L" Solution

\begin{tabular}{|c|c|c|c|c|c|c|c|c|}
\hline & & & & & גp & $\begin{array}{l}\text { zer per } \\
\text { group }\end{array}$ & $\begin{array}{c}\max \text { flux } \\
\text { change }\end{array}$ & $\begin{array}{c}\text { at } \\
\text { mesh }\end{array}$ \\
\hline & ${ }^{* *}$ neg or & zero & transport & $f \operatorname{lu} x * \star \star$ & 69 & 38 & $0.67 \mathrm{E}-04$ & 95, \\
\hline & **neg or & zero & transport & flux $* \star *$ & 68 & 43 & $0.93 E-04$ & 99 \\
\hline
\end{tabular}




\begin{tabular}{|c|c|c|c|c|c|}
\hline * & $* *$ neg or zero transport flux $* * *$ & 67 & 44 & $0.74 \mathrm{E}-04$ & 99,208 \\
\hline * & $* *$ neg or zero transport flux $* * *$ & 66 & 56 & $0.50 \mathrm{E}-04$ & 32,80 \\
\hline * & $* *$ neg or zero transport flux $* * *$ & 65 & 55 & $0.80 \mathrm{E}-04$ & 29, \\
\hline * & $* \star$ neg or zero transport flux $* * *$ & 64 & 69 & $0.67 \mathrm{E}-04$ & 53,36 \\
\hline * & * possible slow dsa accel. $* * * * *$ & 63 & 113 & $0.77 \mathrm{E}-04$ & 61, \\
\hline * & $* *$ possible slow dsa accel. $* * * * *$ & 62 & 56 & $0.64 \mathrm{E}-04$ & 23 \\
\hline * & $\star *$ possible slow dsa accel. $* * * \star *$ & 61 & 49 & $0.63 E-04$ & 99,23 \\
\hline * & ** possible slow dsa accel. $* * * \star *$ & 60 & 38 & $0.78 \mathrm{E}-04$ & 99,12 \\
\hline * & $\star *$ possible slow dsa accel. $* * \star \star \star *$ & 59 & 39 & $0.15 E-04$ & 99, \\
\hline * & 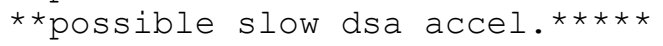 & 58 & 23 & $0.92 \mathrm{E}-04$ & 97, \\
\hline * & $\star *$ possible slow dsa accel. $* * * \star *$ & 57 & 18 & $0.45 E-04$ & 99, \\
\hline * & 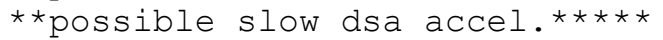 & 56 & 16 & $0.50 E-04$ & 86, \\
\hline * & $* *$ possible slow dsa accel. $* * * * *$ & 55 & 19 & $0.72 \mathrm{E}-04$ & 65, \\
\hline * & & 54 & 13 & $0.93 E-04$ & 78,15 \\
\hline * & & 53 & 10 & $0.17 E-04$ & 56, \\
\hline * & & 52 & 9 & $0.14 \mathrm{E}-04$ & 74,21 \\
\hline * & & 51 & 9 & $0.55 E-04$ & 46, \\
\hline * & & 50 & 8 & $0.74 E-04$ & 99,26 \\
\hline * & & 49 & 8 & $0.77 E-05$ & 37,4 \\
\hline * & & 48 & 7 & $0.73 E-04$ & 33,5 \\
\hline * & & 47 & 8 & $0.47 E-05$ & 88,6 \\
\hline * & & 46 & 20 & $0.61 \mathrm{E}-04$ & 11,232 \\
\hline * & & 45 & 14 & $0.71 E-04$ & 6,11 \\
\hline * & & 44 & 14 & $0.65 E-04$ & 6, \\
\hline * & & 43 & 17 & $0.79 E-04$ & 6,114 \\
\hline * & $* *$ possible slow dsa accel. $* * * * *$ & 42 & 22 & $0.73 E-04$ & 8,107 \\
\hline * & & 41 & 21 & $0.63 E-04$ & 9,109 \\
\hline * & & 40 & 15 & $0.92 \mathrm{E}-04$ & 7,116 \\
\hline * & & 39 & 12 & $0.80 \mathrm{E}-04$ & 6,111 \\
\hline * & & 38 & 12 & $0.82 \mathrm{E}-04$ & 6,11 \\
\hline * & & 37 & 12 & $0.95 E-04$ & 6, \\
\hline * & & 36 & 12 & $0.66 \mathrm{E}-04$ & 6 , \\
\hline * & & 35 & 13 & $0.87 \mathrm{E}-04$ & 5, \\
\hline * & & 34 & 10 & $0.99 E-04$ & 95,232 \\
\hline * & & 33 & 12 & $0.83 E-04$ & 6, \\
\hline * & & 32 & 13 & $0.68 E-04$ & 6, \\
\hline * & & 31 & 13 & $0.56 \mathrm{E}-04$ & 6, \\
\hline * & & 30 & 12 & $0.55 E-04$ & 95,232 \\
\hline * & & 29 & 11 & $0.43 E-04$ & 6 \\
\hline * & & 28 & 10 & $0.79 E-04$ & 95,232 \\
\hline * & & 27 & 10 & $0.41 E-04$ & 38,25 \\
\hline * & & 26 & 11 & $0.55 E-04$ & 22,139 \\
\hline * & & 25 & 10 & $0.69 E-04$ & 99,232 \\
\hline * & & 24 & 9 & $0.90 E-04$ & 99,232 \\
\hline * & & 23 & 11 & $0.51 E-04$ & 73,232 \\
\hline * & & 22 & 10 & $0.85 E-04$ & 73,232 \\
\hline * & & 21 & 11 & $0.41 E-04$ & 99,232 \\
\hline * & & 20 & 11 & $0.72 \mathrm{E}-04$ & 75,232 \\
\hline * & & 19 & 12 & $0.47 E-04$ & 1,98 \\
\hline * & & 18 & 11 & $0.60 \mathrm{E}-04$ & 99,229 \\
\hline * & & 17 & 13 & $0.45 E-04$ & 99,22 \\
\hline * & & 16 & 15 & $0.47 E-04$ & 99,228 \\
\hline * & & 15 & 16 & $0.61 E-04$ & 1,96 \\
\hline * & & 14 & 16 & $0.51 E-04$ & 1,9 \\
\hline * & & 13 & 17 & $0.92 E-04$ & 1,9 \\
\hline * & & 12 & 18 & $0.95 E-04$ & 1,9 \\
\hline * & & 11 & 19 & $0.53 E-04$ & 1,87 \\
\hline * & & 10 & 18 & $0.96 E-04$ & 1,9 \\
\hline$\star$ & & 9 & 18 & $0.74 E-04$ & 1,8 \\
\hline * & & 8 & 18 & $0.67 E-04$ & 1,8 \\
\hline
\end{tabular}




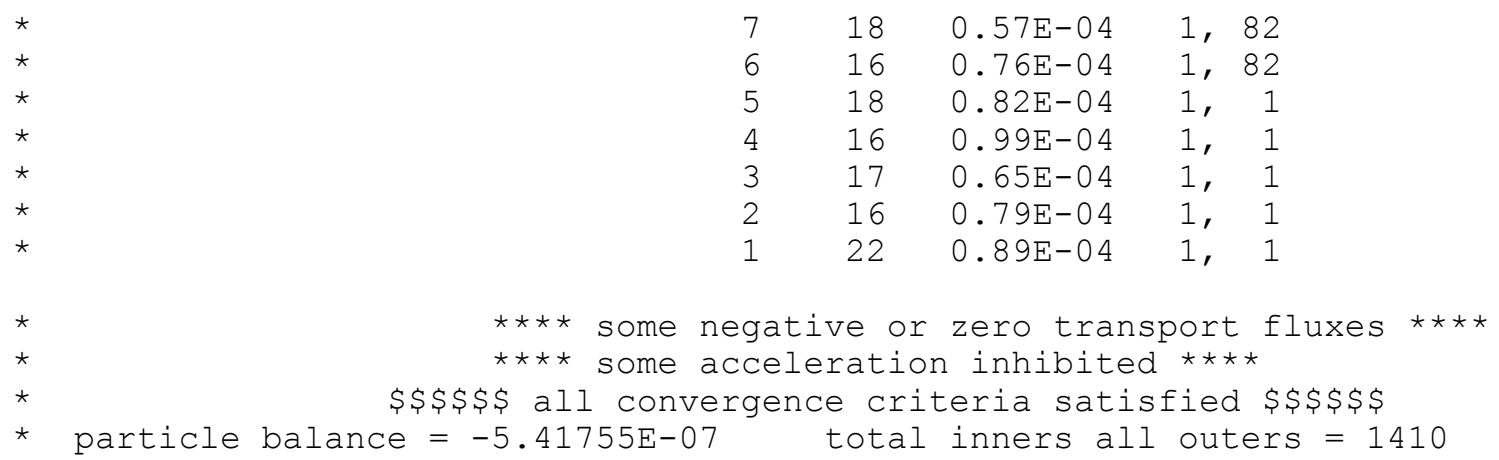




\section{REFERENCES}

1. E. Greenspan, "Developments in Perturbation Theory", Advances in Nuclear Science and Technology., Vol. 9, 181-268, Academic Press, Inc., 1976.

2. Greenspan, "A Method for the Optimization of Fusion Reactor Neutronic Characteristics," Proc. Conf. On Mathematical Models and Computation Techniques for Analysis of Nuclear Systems, Ann Arbor, Michigan, 162-179 (1973). Also in MATT-981.

3. E. Greenspan and W.G. Price, "The Breeding Potential of the Princeton Reference Fusion Power Reactor," Proc. Conf. On the Technology of Controlled Thermonuclear Reactors, San Diego, CA, 10 pages (1974). Also in MATT-1043.

4. D. Gilai, E. Greenspan, P. Levin and W.G. Price, "Optimal Iron-Water Shields for Fusion Reactors," Proc. $5^{\text {th }}$ Int. Conf. Reactor Shielding, CONF-770401, pp. 731-738 (1977).

5. D. Gilai, E. Greenspan and P. Levin, "Optimal W-TiH ${ }_{2}$ Shields for Fusion Reactors," Trans. American Nuclear Society, 45, 625-626 (1983).

6. D. Gilai, E. Greenspan and P. Levin, "On Optimal Shields for Fusion Reactors," Proc. $6^{\text {th }}$ Int. Conf. On Radiation Shielding, Tokyo, Japan, Vol. II, 646-654 (1983).

7. Greenspan and Y. Karni, "High-Energy Multiplication Blankets for Cat-D Fusion Reactors," Trans. American Nuclear Society, 46, 216-217 (1984).

8. D. Gilai, E. Greenspan, P. Levin and Y. Karni, "Optimal Shields for Fusion Reactors," Proc. American Nuclear Society Topical Mtg. On Reactor Physics and Shielding, Chicago, IL (Sept. 1984) pp. 250-260.

9. E. Greenspan, P. Levin and A. Kinrot, "Optimal Shield Concepts for Experimental Fusion Devices," Fusion Technol., 8, 1026-1031 (1985).

10. E. Greenspan, A. Kinrot and P. Levin, "Optimal Blanket Concepts for D-T Fusion Reactors," Fusion Technol., 8, 619-624 (1985).

11. A. Kinrot and E. Greenspan, "Minimum Thickness Lithium-Lead Blankets," Trans. American Nuclear Society, 48, 104-105 (1985).

12. E. Greenspan and Y. Karni, "Tungsten-Based Composite Materials for Fusion Reactor Shields," Trans. American Nuclear Society, 49, 440-441 (1985).

13. A. Kinrot and E. Greenspan, "Borides vs. Hydrides for Minimizing Fusion Reactor Shield Thickness," Trans. American Nuclear Society, 49, 441-442 (1985).

14. E. Greenspan and Y. Karni, "Neutronic Optimization of Li4SiO4/Be/He/SS Blankets and Shields for the NET," Fusion Technology, 10, 1605-1610 (1986).

15. E. Greenspan and Y. Karni, "Minimum Thickness Blankets Using Neutron Multiplying and Hydrogenous Materials," Trans. American Nuclear Society, 54, 133-134 (1987).

16. E. Greenspan and Y. Karni, "Helium Cooled Beryllium Blanket Thickness Dependence on Tritium Breeding Material,” Trans. American Nuclear Society, 55, 124-126 (1987).

17. Y. Karni and E. Greenspan, "Tritium Breeding Possibilities in Aqueous Self Cooled Blankets," Trans. American Nuclear Society, 54, 121-123 (1987).

18. Y. Karni and E. Greenspan, "Minimum Thickness Blanket - Shield for Fusion Reactors," Fusion Eng. And Design, 10, 63-69 (1989).

19. E. Greenspan, J. Bendahan and T. Gozani, "A Novel Approach to Design Optimization of Nuclear-Based Explosive Detection Systems," Proc. $1^{\text {st }}$ Int. Symp. On Explosive Detection, Atlantic City, N.J., November 13-15, 1991.

20. E. Greenspan, "High Effectiveness Shielding Materials and Optimal Shield Design," ASTM Journal of Testing and Evaluation, 20, 71- 77 (1992). 
21. Y. Karni, E. Greenspan, J. Bendahan and T. Gozani, "Effective Materials and Optimal Design for Radiation Shields," Proc. of the American Nuclear Society Topical Mtg. On New Horizons in Radiation Protection and Shielding, Pasco, WA, April 26 - May 1, 1992, pp. 154-161.

22. E.F. Watkins, C.E. Annese and E. Greenspan, "Shield Cost Minimization Using SWAN," Trans. American Nuclear Society, 68, 393-395 (1993).

23. E.F. Watkins and E. Greenspan, "A Perturbation Technique for Shield Weight Minimization," Trans. American Nuclear Society, 68, 326-327 (1993).

24. E. Greenspan et al., "The "Material Effectiveness Function" Approach to SPACE-R Shield Optimization," Proc. of the $11^{\text {th }}$ Symposium on Space Nuclear Power and Propulsion, Albuquerque, NM, Jan. 9-13, 1994, pp. 87-95.

25. Y. Karni and E. Greenspan, "The "Equal System Weight Replacement Effectiveness Function" Approach to Shield Mass Minimization," Proc. $8^{\text {th }}$ International Conference on Radiation Shielding, Arlington, TX, April 24-27, 1994. pp. 866-873.

26. Y. Karni and E. Greenspan, "Medium Density Hydrogenous Materials for Shielding Against Nuclear Radiation," Proc. $8^{\text {th }}$ International Conference on Radiation Shielding, Arlington, TX, April 24-27, 1994. pp. 43-50.

27. Y. Karni and E. Greenspan, "Using "SWAN" for Optimizing the Neutron Source Assembly Design for Boron-Neutron-Capture-Therapy Facilities," Proc. $1^{\text {st }}$ Int. Workshop on Accelerator-Based Neutron Sources for Boron Neutron Capture Therapy, Jackson, WY, Sept. 11-14, 1994. pp. 225-242.

28. Y. Karni, E. Greenspan, J. Vujic and B. Ludewigt, "Optimal Beam-Shaping Assemblies for BNCT Facilities," Trans. American Nuclear Society, 73, 29-30 (1995).

29. E. F. Watkins and E. Greenspan, "The SWAN/NPSOL Code System for Multivariable Multiconstraint Shield Optimization," Trans. American Nuclear Society, 73, 347-349 (1995).

30. E. Greenspan, Y. Karni, J. Vujic and E.F. Watkins, "Minimum Critical Mass of ${ }^{239} \mathrm{Pu}$ in Rock,"Proc. ANS Embedded Topical Mtg. On DOE Spent Nuclear Fuel and Fissile Material Management, Reno, NV, June 16-20, 1996. pp. 281-289.

31. Y. Karni and E. Greenspan, "The SWAN Code for Optimization of Critical Systems," Trans. American Nuclear Society, 76, 242 (1997).

32. Y. Karni and E. Greenspan, "Subcritical Multiplication of Accelerator Generated Neutrons for BNCT,” Trans. American Nuclear Society, 76,4 (1997).

33. Y. Karni and E. Greenspan, "The SWAN Code for Minimum Critical Mass and Maximum $\mathrm{k}_{\text {eff }}$ Determination," Proc. ANS Topical Meeting on Criticality Safety Challenges in the Next Decade, Chelan, WA, Sept. 7-11, 1997. pp. 181-188.

34. Y. Karni, E. Greenspan and J. Vujic, "Accelerator Driven Fission Neutron Source for BNCT," Proc. ANS Embedded Topical Mtg. On Accelerator Applications, Albuquerque, N.M., Nov. 1997. pp 508-515.

35. E. Greenspan, Y. Karni, J. Vujic and B. Ludewigt, "BNCT Beam Quality Sensitivity to Source Neutron Spectrum," Trans. American Nuclear Society, 77, 44 (1997).

36. Y. Karni, E. Greenspan, and J. Vujic, "Perturbation Theory Guided Design of Beam Shaping Assemblies," Trans. American Nuclear Society, 77, 354-355 (1997).

37. Y. Karni, E. Greenspan and J. Vujic, "BNCT Beam Quality Sensitivity to BSA Thickness," Trans. American Nuclear Society, 78, 19-20 (1998). 
38. E. Greenspan and Y. Karni, "BNCT Beam Quality Maximization", Proc. $8^{\text {th }}$ Int. Symp. on Neutron Capture Therapy for Cancer, La Jolla, CA, Sept. 13-18, 1998.

39. E. Greenspan and Y. Karni, "The SWAN Approach to Neutron Beam Optimization for BNCT and other Applications" Proc. Int. Conf. on the Physics of Nuclear Science and Technology, Long Island, N.Y., Oct. 5-8, 1998.

40. W.W. Engle, Jr., "A User's Manual for ANISN, a One Dimensional Discrete Ordinates Transport Code with Anisotropic Scattering,“ Oak Ridge Gaseous Diffusion Plant Report K-1693 (1967). See also RSIC Computer Code Collection CCC-82.

41. W.A. Rhoades and R.L. Childs, "The DORT Two Dimensional Discrete Ordinates Transport Code, Nucl. Sci. \& Eng., 99, 1, 88 (1988). See also RSIC Computer Code Collection CCC-543.

42. The GIP program for Preparation of Group-Organized Cross-Section Libraries" Edited by W.A. Rhoades on Apr-13, 1978, pp 94-100 of: W. A. Rhoades "DOT-IV - Two Dimensional Discrete Ordinates Radiation Transport Code System", CCC-320 of ORNL , October 1979.

43. E. Greenspan, Y. Karni, L.M. Petrie and D. Regev, "SWANS: A Prototypic SCALE Criticality Sequence for Automated Optimization Using the SWAN Methodology," ORNL/TM-1999/274 (UCBNE-4226), Oct. 2000. 


\section{PART II}

\section{Identification of the Maximum Attainable Beam Quality for BNCT}

It has recently been shown ${ }^{37}$ that (1) the success probability of Boron Neutron Capture Therapy (BNCT) very strongly depends on the clinical quality of the neutron beam (as well as on the boron concentration in the tumor and normal tissue), and (2) the neutron beam quality available from three reactors with existing facilities for epithermal neutron BNCT is not satisfactory, especially for deep tumors. Consequently, for a given boron compound and a neutron source, the success probability of the BNCT strongly depends on how close to optimal nuclear engineers can design the Beam Shaping Assembly (BSA) to be.

It has also been shown that (1) the beam quality strongly depends on the BSA design (See, for example, Refs. 28, 35, 38-40), and (2) the SWAN code is uniquely suitable and unmatchable by any other code for (a) identifying the maximum beam quality attainable from a given neutron source $^{27}$ and (b) systematic comparison of the viability of different neutron sources for BNCT ${ }^{28,}$ $32,34,35$. In fact, in the limited application of SWAN to BSA optimization we made a number of useful discoveries: (1) ${ }^{7} \mathrm{LiF}^{27,28}$ and $\mathrm{MgF}_{2}{ }^{36}$ are very promising major constituents for BSA (offering a better beam quality than $\mathrm{AlF}_{3} / \mathrm{Al}$ ); (2) The beam quality attainable from a hard spectrum neutron source can be similar to that attainable from soft spectrum neutron source ${ }^{28,35}$; (3) The optimal composition of the BSA for hard spectrum neutron source is quite complex; it may consist of 4 to 5 materials (excluding the collimator materials) arranged in highly heterogeneous geometry ${ }^{28,}{ }^{34}$. Figure 4 of Ref. 34 shows, for illustration, the BSA SWAN identified for a $\mathrm{Be}(19 \mathrm{MeV} \mathrm{p}, \mathrm{n})$ source. It is almost inconceivable that such a design could have arrived at without SWAN. Not only SWAN identified the desirable constituents, but it also tells that no other constituent, out of the dozen or so considered, can further improve the beam quality.

Although the 1-D SWAN can show trends and discover promising design approaches and BSA constituents, it can not simulate the BNCT facility as accurate as needed for reliable design. Its primary deficiencies are inability to (a) well represent the brain and tumor size and geometry, and to account for (b) the reflector, and (c) the collimator.

The objective of PART II of the study is to perform a comprehensive systematic study aimed at (1) finding the maximum beam quality which can be attained from different neutron sources, and (2) quantifying the corresponding dose-equivalent to be delivered to the tumor, per source neutron, and (3) identifying the optimal BSA design. Additional objective is to find the sensitivity of the attainable beam quality and of the optimal BSA design to the boron concentration and to the tumor location. The original plan of this study was to use the 2-D SWAN developed in PART I of the study for PART II. In view of the convergence difficulties encountered with the 2-D SWAN we could not apply it to PART II. However related studies were carried-out during this project period by UC Berkeley students. These studied used Monte Carlo codes rather than the 2D-SWAN; they were financially supported by the Lawrence Berkeley National Laboratory. LBNL personnel participated in these studies. Prof. Jasmina 
Vujic, this project PI, served as the academic advisor to these students. A couple of papers summarizing this LBNL sponsored work are included in Appendix A.

\section{PART III}

\section{Feasibility Assessment of an Accelerator-Driven Subcritical Fission Neutron Source}

Accelerator-based neutron source, if sufficiently intense to enable acceptable patient treatment time and not prohibitively expensive, can be beneficial in many hospitals around the country for BNCT, other neutron treatments, and short lived medical radio-isotope production. Although the state-of-the-art of accelerators and beam target technologies is close to that required for commercial BNCT facility, additional R\&D effort is required (primarily to increase the ion beam current and the heat flux which targets can reliably accommodate for long duration).

Nuclear reactors of state-of-the-art technology can provide intense enough neutron beams. However, they are relatively expensive to construct and run, and cannot be sited in or near hospitals.

We have recently proposed ${ }^{32,34}$ that a proper combination of a small accelerator and a small Subcritical fission Assembly (SA) could be constructed using state-of-the-art technology, may be sited in hospitals, and are likely to be more economical than either critical reactors or accelerator only neutron sources. Following are selected conclusions from our preliminary feasibility assessment $^{34}$ : (1) It is possible to design a BSA for a fission neutron source that will provide a beam quality which is comparable to that attainable when using the lower energy neutrons obtained from the $2.5 \mathrm{MeV} \mathrm{p}-{ }^{7} \mathrm{Li}$ reaction (System A). (2) The required beam current for System A can be reduced by a factor of 2 or 3.6 with use of, respectively, a thermal or a fast SA. The corresponding reduction in the current required from $19 \mathrm{MeV} \mathrm{p}$ accelerator and a Be target (System B) is a factor of 12 or 46. (3) Relative to the beam current required for System A with no SA, the current required for System B with a fast SA is lower by a factor of 88 or 340 ! (4) The total thermal power (from beam + fission) which need be removed from the SA is on the order of $10 \mathrm{~kW}$ (as compared with $\sim 50 \mathrm{~kW}$ from a much smaller target of accelerator only facility and versus several hundreds to thousands of $\mathrm{kW}$ for critical reactors). (5) Use of a SA may significantly reduce the treatment cost per patient. It may also reduce the time and cost required for the development of commercial accelerator driven BNCT facilities. (6) It appears that SAs could be designed to be inherently safe against criticality which might endanger the patients or the staff. (7) It is likely that SAs and BSAs that are more attractive than those considered in Ref. 34 could be developed.

The objective of Part III of this project was to perform a more thorough assessment of the feasibility of accelerator driven SA (ADSA) neutron source for BNCT applications. If ADSA will be found as attractive as indicated in Ref. 34, neutron therapies could be offered to the public in hospitals around the country sooner, and at a relatively lower cost than possible 
otherwise. If successfully commercialized, ADSA facilities may also be a new US export product and a new noble use of fission.

The original plan of this study was to use the 2-D SWAN developed in PART I of the study for PART III. In view of the convergence difficulties encountered with the 2-D SWAN we could not apply it to PART III. However a related study was carried out by UC Berkeley undergraduate students in the framework of the NE-170 Nuclear Design course project taught by Professor Ehud Greenspan - a co-PI of this project. This study used the Monte Carlo code MCNP rather than the 2D-SWAN. A summary report of this student project is included in Appendix B. This report was originally prepared for submission to the American Nuclear Society Students Design Competition. Our students won the first place in this year contest. 


\title{
APPENDIX A \\ UC BERKELEY STUDIES RELATED TO MAXIMUM ATTAINABLE BEAM QUALITY AND OPTIMAL BEAM SHAPING ASSEMBLY DESIGNS
}

\author{
See Attachment: \\ J.M. Verbeke, J. Vujic, and K.N. Leung, "Neutron Beam Optimization for BNCT using the \\ D-D and D-T High-Energy Neutron Sources," Nucl. Technol. 129 (2), 257-278 (2000)
}

\begin{abstract}
SUMMARY
A mono-energetic neutron beam simulation study has been carried out to determine which neutron energy is the most suitable for treatment of shallow and deep-seated brain tumors in the context of Boron Neutron Capture Therapy. For deep-seated tumors, the energy range 1 to 20 $\mathrm{keV}$ appears to be optimal, with a maximum therapeutic gain at $\sim 8 \mathrm{keV}$. For shallow tumors, any neutron beam of energy higher than 1 to $10 \mathrm{eV}$ and lower than $\sim 8 \mathrm{keV}$ will result in a relatively high therapeutic gain. However, it should be noted that even if the main tumor mass is shallow, microscopic fingerlets spreading throughout the surrounding tissues can reach greater depths. Since these fingerlets also have to be irradiated, $8 \mathrm{keV}$ neutron beams should be preferred for treatment of shallow tumors. An overall higher therapeutic gain is obtained with such neutron beams.
\end{abstract}

The neutron beam diameter has a considerable effect on the tumor dose for deep-seated tumors. For instance, the tumor dose at a depth of $8 \mathrm{~cm}$ more than doubles when the neutron beam diameter increases from $6 \mathrm{~cm}$ to $18 \mathrm{~cm}$. The drawback of larger diameter beams is that the volume of high healthy tissue dose also increases with the beam diameter. For shallower tumors, the increase in tumor dose with beam diameter is still present but less pronounced, and it vanishes for 2-cm-deep tumors. However, the volume of high healthy tissue dose still increases with the beam diameter. From the results of this neutron beam energy and diameter simulation study, we can conclude that (a) $8 \mathrm{keV}$ neutron beams are optimal for deep-seated tumors, but (b) no set of neutron beam diameter and energy is best in all respects for any kind of tumors. Diameter and energy of the neutron beam have to be determined by the characteristics of the tumor to be treated, such as tumor depth, size and how far from the main tumor mass the fingerlets spread. Given all these characteristics, simulation of particle transport in the brain can help determine the optimal set of neutron beam diameter and energy for treatment.

Based on the results of this simulation study, the fusion reactions D-D and D-T have then been investigated as neutron sources for BNCT. Two different methods are used to compute the dose distribution in the brain. The first method is based on the simulation of the radiation transport in the BSA and the phantom with MCNP. The second method is based on the coupling of MCNP and BNCT RTPE for the radiation transport simulations in the BSA and phantom respectively, 
the coupling being done through an exit window between the BSA and the phantom. The radiation transmitted through the delimiter is not accounted for in the second method, due to the limitations of BNCT RTPE, which makes it less accurate than the first one. The first method, although more accurate is much more time-consuming due to the use of MCNP to simulate radiation transport through both the BSA and the phantom head.

Our analysis shows that the low neutron yield of the D-D reaction is an obstacle for the treatment of Glioblastoma Multiforme. On the other side, high-energy neutrons from D-T can be moderated to the desired energy range without reducing the neutron flux to a negligible level. With the optimal moderator and lead reflector configuration, a 1 A mixed deuteron/triton beam with energy of $150 \mathrm{keV}$ accelerated onto a titanium target leads to a treatment time of 1 hour. The dose near the center of the brain obtained with this configuration is more than $65 \%$ higher than doses obtained by a neutron beam currently used at BMRR for clinical trials, and comparable to other accelerator-based neutron sources. A multiple beam configuration could increase the tumor dose at the center of the brain and reduce the treatment time. 


\section{APPENDIX B}

\section{FEASIBILITY ASSESSMENT OF AN ACCELERATOR-DRIVEN SUBCRITICAL FISSION NEUTRON SOURCE}

\section{SUMMARY}

A compact Boron Neutron Capture Therapy (BNCT) facility was designed using a Deuterium-Deuterium (D-D) fusion neutron source and a Subcritical Fission Multiplier (SCM). BNCT is a bi-modal approach utilizing external neutrons that are absorbed in tumor-seeking borated pharmaceuticals. Neutron absorption in the boron compounds release charged particles via the ${ }^{10} \mathrm{~B}(\mathrm{n}, \alpha){ }^{7} \mathrm{Li}$ reaction, resulting in a localized dose to the surrounding cancerous tissue. This compact facility could be easily deployed in hospitals to treat glioblastoma multiforme, a fatal, highly non-localized brain cancer that affects about six thousand people every year in the United States alone. Other applications include synovectomies, the treatment of head and neck cancers, and the treatment of melanoma.

The source of neutrons is a novel, highly compact, coaxial electrostatic accelerator under development by Prof. K.N. Leung and his group at the Lawrence Berkeley National Laboratory with the participation of UCB NE students. The neutron source dimensions are approximately $26 \mathrm{~cm}$ in diameter and $21 \mathrm{~cm}$ in length. Previous work by J. Verbeke of UCB NE concluded that the neutron source intensity generated by accelerating deuterium and tritium is sufficient for BNCT applications. There are a number of disadvantages of using tritium for BNCT: it is expensive, radioactive, needs frequent refueling and difficult to manage. However, this accelerator can generate neutrons by accelerating only deuterium ions, but the intensity is approximately 30 times too low. The goal of the project was to assess the feasibility of augmenting the D-D fusion neutron source intensity by a factor of at least 30 using a small, inherently safe, subcritical fission assembly.

The subcritical multiplier (SCM) has a core that is $80 \mathrm{~cm}$ in diameter and $14 \mathrm{~cm}$ thick. It is made of zircaloy clad uranium dioxide fuel and water moderator/coolant. The water-to-fuel volume ratio was optimized to give the maximum $\mathrm{k}_{\mathrm{eff}}$ so that any change from nominal operating conditions would result in a loss in reactivity. The uranium enrichment for the desired $\mathrm{k}_{\text {eff }}$ of 0.98 was found to be $3.8 \%{ }^{235} \mathrm{U}$ and the optimal moderator-to-fuel volume ratio was found to be $2.25: 1$. The neutron current coming out of the SCM is 30 times higher than the D-D neutron current entering the SCM from the neutron source. The fission power of the SCM is $\sim 500 \mathrm{~W}$ - approximately $0.25 \%$ of the accelerator power. Only $\sim 0.004 \%$ of the ${ }^{235} \mathrm{U}$ is consumed per year of operation assuming a capacity factory of $100 \%$.

Thermal-hydraulic analysis was performed to ensure the integrity of the fuel, cladding, and shielding of the SCM by passive means under the most severe plausible accidents. With SCM power levels well below $1 \mathrm{~kW}$, the free convection of light water between fuel plates was found to be sufficient to cool the SCM. Even if this mechanism were to fail in the event of a loss-of-coolant-accident, the system is designed such that conduction through the SCM and free convection of air or water is sufficient for assuring the integrity of the SCM fuel, reflector and shield.

The total activity of the fission products generated in the SCM during 10 years of full power operation is 625 Curie 3 days after shutdown. In comparison, the activity of the tritium in a CNS based on D-T fusion giving comparable neutron beam intensity is 2230 Curies. Whereas the fission products are imbedded and well contained in the clad solid fuel matrix, the tritium is in gaseous form, highly permeable and difficult to contain. The cost of the SCM is estimated to be approximately $\$ 250 \mathrm{~K}$. This is just about the cost of T 
for one full power year of operation of a D-T neutron source giving a comparable intensity of a neutron beam of comparable quality.

It is concluded that use of a small subcritical neutron multiplier in combination with a compact D-D neutron source could provide a compact BNCT facility that is passively safe and economical. However, a more thorough study is needed before commercialization of compact, safe, and possible mobile intense neutron irradiation-facilities that will be affordable to hospitals, research laboratories, industry and universities around the country. 


\title{
A COMPACT BNCT FACILITY USING A FUSION NEUTRON SOURCE AND A FISSION MULTIPLIER
}

\author{
NE-170 Nuclear Design Project \\ Advisor: E. Greenspan
}

May 2002

\author{
D. Chivers, S. Guess, L. Kim, W. Waldron, Y. Zhu \\ Department of Nuclear Engineering \\ University of California, Berkeley \\ Berkeley, CA 94720-1730
}

\begin{abstract}
A compact Boron Neutron Capture Therapy (BNCT) facility was designed using a DeuteriumDeuterium (D-D) fusion-based Compact Neutron Source (CNS) and a Subcritical Fission Multiplier (SCM). BNCT is a bi-modal cancer therapy utilizing external neutrons that are absorbed in tumor-seeking borated pharmaceuticals. Neutron absorption in the boron compounds release charged particles via the ${ }^{10} \mathrm{~B}(\mathrm{n}, \alpha)^{7} \mathrm{Li}$ reaction, resulting in a localized dose to the surrounding cancerous tissue. This compact facility could be easily deployed in hospitals to treat glioblastoma multiforme, a fatal, highly non-localized brain cancer that affects about six thousand people every year in the United States alone. ${ }^{\text {i }}$ Other applications include synovectomies, the treatment of head and neck cancers, and the treatment of melanoma.

Previous work by Verbeke ${ }^{\text {ii }}$ (See also Appendix A) demonstrated that a D-D fusion CNS-based facility was capable of treating a patient in 30 hours, far longer than the desired treatment time of one hour or less. To improve this facility, a subcritical fission multiplier assembly was designed to amplify the neutron intensity and reduce treatment times. The resulting fission neutrons are then filtered through a Beam Shaping Assembly (BSA) to optimize the energy spectrum. A novel method of delivering boron-containing compounds was also researched, but will not be discussed in this report pending the results of that study.
\end{abstract}

The neutron source is based on a highly compact coaxial electrostatic accelerator under development at the Lawrence Berkeley National Laboratory. This cylindrical source consists of a radio frequency driven deuterium plasma. The atomic deuterium ions are then extracted to a deuterium-loaded target where the beam drives the reaction and continuously reloads the target. A vacuum contained within a quartz vacuum chamber is used for high voltage insulation around the target cylinder. With a $200 \mathrm{kV}$ extraction voltage and a $1 \mathrm{~A}$ beam current, this source emits $2.45 \mathrm{MeV}$ neutrons isotropically at about $10^{12}$ neutrons per second. The outer dimensions of the source are approximately $26 \mathrm{~cm}$ in diameter and $21 \mathrm{~cm}$ in length. Cooling of the CNS is achieved by forced annular flow around the cylindrical target. Such an active cooling system is sufficient since the CNS does not pose major safety risks.

A reflector assembly surrounding the source reflects neutrons towards the SCM with shielding provided by an outer neutron-absorbing layer. Using MCNP, infinite slabs of varying thickness 
were modeled to determine the fraction of source neutrons reflected into the SCM. Various materials, including light water, heavy water, lead, and graphite were explored. Ultimately, lead was selected given its high reflectivity and its minimal impact on the neutron energy spectrum. A similar study was performed to characterize the shielding properties of lithiated polyethylene and light water. Lithiated polyethylene was selected given its ability to moderate and absorb neutrons without emitting gamma rays.

To amplify the neutrons from the CNS, a subcritical fission assembly consisting of Zircaloy cladding, uranium dioxide, light water, and a lead reflector was designed with MCNP. The design goal was a $\mathrm{k}_{\mathrm{eff}}$ of 0.98 , which corresponds to a multiplication factor of 50 . The moderatorto-fuel volume ratio was optimized to maximize $\mathrm{k}_{\infty}$. Varying the SCM dimensions and uranium enrichment maximized the neutron current into the Beam Shaping Assembly.

The optimal moderator-to-fuel volume ratio was found to be about 2.25:1. The optimal fission plate dimensions are $80 \mathrm{~cm}$ in diameter and about $14 \mathrm{~cm}$ in length with five $1 \mathrm{~cm}$ thick $3.8 \mathrm{w} / \mathrm{o}$ enriched plates. The $\mathrm{k}_{\mathrm{eff}}$ of this system is 0.983 resulting in about 30.4 neutrons leaking into the BSA per source neutron. The radial leakage of neutrons accounts for the discrepancy between the observed value and the expected multiplication factor of 50 .

In contrast to the CNS, passive mechanisms are desired for cooling the SCM as the integrity of the fuel, cladding, and shielding must be ensured under the most severe plausible accidents. With SCM power levels well below $1 \mathrm{~kW}$, the free convection of light water between fuel plates is sufficient to cool the SCM. Even if this mechanism were to fail in the event of a loss-of-coolantaccident, the system is designed such that conduction through the SCM and free convection of air or water is sufficient for cooling.

A Beam Shaping Assembly (BSA) is required to moderate and shape the neutron spectrum emanating from the SCM. To maximize the tumor dose while minimizing undesirable effects on healthy tissue, the most effective neutron energies are between 1 and $20 \mathrm{keV}$. Using MCNP, the effects of various materials upon neutron spectrums were analyzed. Materials studied included lead, iron, Fluental $\left(40 \% \mathrm{Al} / 60 \% \mathrm{AlF}_{3}\right.$ mixture), and ${ }^{6} \mathrm{LiF}$. Analyzing MCNP results and neutron cross-section data, a $45 \mathrm{~cm}$ thick Fluental layer combined with a $0.1 \mathrm{~cm}$ thick ${ }^{6} \mathrm{LiF}$ thermal neutron filter was optimal for a $80 \mathrm{~cm}$ diameter cylindrical BSA.

Given time constraints, the patient dose and treatment times are not accurately quantified at this time. While preliminary data is available, additional simulations are required to reduce statistical error and publishing these vales is premature. 


\section{INTRODUCTION}

A compact Boron Neutron Capture Therapy (BNCT) facility was designed using a DeuteriumDeuterium (D-D) fusion-based Compact Neutron Source (CNS) and a Subcritical Fission Multiplier (SCM). BNCT is a bi-modal cancer therapy utilizing external neutrons that are absorbed in tumor-seeking borated pharmaceuticals. Neutron absorption in the boron compounds release charged particles via the ${ }^{10} \mathrm{~B}(\mathrm{n}, \alpha)^{7} \mathrm{Li}$ reaction, resulting in a localized dose to the surrounding cancerous tissue. This compact facility could be easily deployed in hospitals to treat glioblastoma multiforme, a fatal, highly non-localized brain cancer that affects about six thousand people every year in the United States alone. ${ }^{\text {iii }}$ Other applications include synovectomies, the treatment of head and neck cancers, and the treatment of melanoma.

Previous work by Verbeke ${ }^{\text {iv }}$ demonstrated that a D-D fusion CNS-based facility was capable of treating a patient in 30 hours, far longer than the desired treatment time of one hour or less. To improve this facility, a subcritical fission multiplier assembly was designed to amplify the neutron intensity and reduce treatment times. The resulting fission neutrons are then filtered through a Beam Shaping Assembly (BSA) to optimize the energy spectrum. A novel method of delivering boron-containing compounds was also researched, but will not be discussed in this report pending the results of that study.

The design of this complex facility was broken up into separate parts (CNS, SCM, BSA, thermal hydraulics) and analysis on each section was carried out in parallel. Interfaces between the sections were approximated in various ways as described within the report. Feedback between the different design teams modified the initial assumptions and imposed new design constraints. Based on these parallel efforts, a complex integrated model was then constructed and analyzed. Ultimately, dose and treatment time are the figures of merit.

\section{Design Goals}

\section{COMPACT NEUTRON SOURCE (CNS)}

A compact, high intensity source of epithermal neutrons is desired for Boron Neutron Capture Therapy (BNCT) as patient dose and treatment time are dependent on neutron source intensity and spectrum. Ideally, the source intensity should be as high as reasonably achievable while the source spectrum, which will be shaped through the device, should be of higher energy than the ideal energies between 1 and $20 \mathrm{keV}$.

\section{Source Options}

One well-developed source is a $2.5 \mathrm{MeV}$ electrostatic proton accelerator that drives a ${ }^{7} \mathrm{Li}$ target to produce neutrons. Unfortunately, a fairly large accelerator is required to hold off the high voltage required for a $2.5 \mathrm{MeV}$ beam. Commercially available proton cyclotrons are another possibility, but they produce currents that are much too low for a reasonable intensity. 
In contrast, a much smaller electrostatic accelerator is under development that accelerates a deuteron beam into a tritium-loaded target, producing neutrons via D-T fusion. While this design is compact due to a lower operating voltage, the safety requirements for handling the tritium inventory makes this design less desirable. However, a similar source based on D-D fusion can be used as it simplifies the handling of materials. Unfortunately, Verbeke has demonstrated that intensities from a deuterium-based source are insufficient, resulting in treatment times around 30 hours. ${ }^{\mathrm{V}}$ However, a subcritical fission multiplier may amplify the source intensity by as much as a factor of fifty, making this D-D source an attractive option for BNCT.

\section{Neutron Source Description}

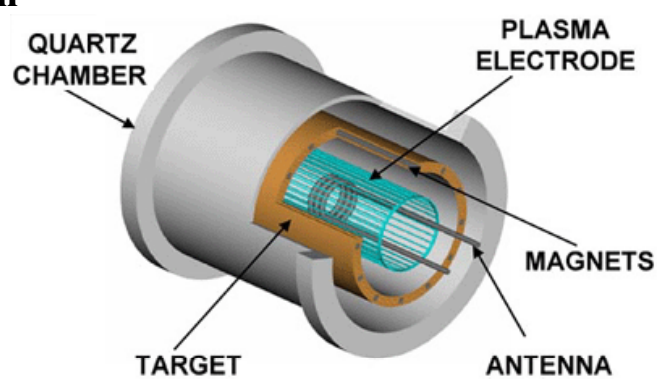

Figure 1 Diagram of Compact Neutron Source.

The neutron source is based on a highly compact coaxial electrostatic accelerator under development at the Lawrence Berkeley National Laboratory. This cylindrical source consists of a radio frequency driven deuterium plasma. The atomic deuterium ions are then extracted to a deuterium-loaded target biased at $-200 \mathrm{kV}$ where the deuterium beam drives the reaction and continuously reloads the target. A vacuum contained within a quartz vacuum chamber is used for high voltage insulation around the target cylinder. The vacuum line, RF power, cooling water, and pulsed high voltage are brought in through a vacuum feed-through at one end of the cylindrical assembly. Any maintenance would involve the movement of reflecting and shielding materials to access the neutron source. With a $200 \mathrm{kV}$ extraction voltage and a $1 \mathrm{~A}$ beam current, this source produces an isotropic source of $2.45 \mathrm{MeV}$ neutrons at about $10^{12}$ neutrons per second. The outer dimensions of the source are approximately $26 \mathrm{~cm}$ in diameter and $21 \mathrm{~cm}$ in length.

The cylindrical geometry of the source is advantageous for holding high voltage due to the uniform distribution of the electric field equipotential lines between the electrode and target cylinders. There are no sharp corners or edges that can cause field enhancements that may lead to electron emission and voltage breakdown. This geometry also simplifies the beam optics such that the beam power is evenly distributed over the cylindrical target, simplifying heat removal. Because this target is wrapped around the source of deuterium ions, there is a large target area in a small space compared to an accelerator with flat plate target. This geometry also leads to a very high current limit as a result of the large beam extraction area. Using a maximum current density of $100 \mathrm{~mA} / \mathrm{cm}^{2}$, the current limit for this design is approximately $27 \mathrm{~A} .^{\mathrm{vi}}$

$\mathrm{I}_{\max }=\pi$ (electrode diameter)(electrode length)(electrode transparency)(current density)

$\mathrm{I}_{\max }=\pi(10.4 \mathrm{~cm})(21 \mathrm{~cm})(0.4)(100 \mathrm{~mA} / \mathrm{cm} 2)=27.44 \mathrm{~A}$

This is a considerable improvement when compared to a source where only one side of the plasma is used for extraction. 


\section{D-D and D-T Reactions}

Although the neutron production cross section for a D-D reaction is approximately two orders of magnitude lower than the cross section for D-T reactions in the 100 to $200 \mathrm{KeV}$ range, D-D was chosen because of the cost and safety concerns regarding tritium. By eliminating the tritium inventory associated with a D-T source, the maintenance and disposal cost is significantly reduced and the handling of the source hardware is simpler and safer. The mechanical design of the system also becomes easier without the need for a sealed system designed to prevent the release of tritium. The neutronics of a D-D system is also desirable as the subcritical multiplier (SCM) has a higher fission cross-section for thermal neutrons and is therefore more efficient when driven at thermal energies. Less moderation, and therefore less absorption, is needed when using the $2.5 \mathrm{MeV}$ neutrons from the D-D reaction compared to the $14 \mathrm{MeV}$ neutrons from the D-T reaction.

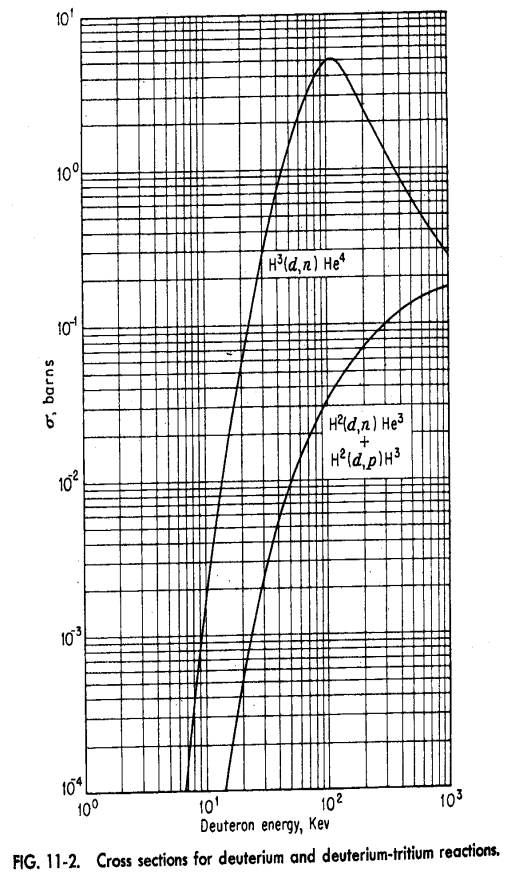

Figure 2 Cross-sections of D-D and D-T reactions. ${ }^{\text {vii }}$

Because the neutron production cross-section increases nonlinearly with deuteron beam energy and linearly with current, it is advantageous to increase the voltage on the extraction electrode as high as possible before increasing the current. This will maximize the intensity per unit beam power $(\mathrm{n} / \mathrm{s} / \mathrm{W})$. For example, the neutron yield from a $1 \mathrm{~A}$ deuteron beam is $2.3 \times 10^{11} \mathrm{n} / \mathrm{s}$ at 100 $\mathrm{KeV}$ and $11.5 \times 10^{11}$ at $200 \mathrm{KeV}$. This corresponds to $2.3 \times 10^{6} \mathrm{n} / \mathrm{s} / \mathrm{W}$ for $100 \mathrm{KeV}$ beam energy compared to $5.75 \times 10^{6} \mathrm{n} / \mathrm{s} / \mathrm{W}$ for $200 \mathrm{KeV}$ beam energy (see Figure 3). It is difficult to work with voltages beyond $200 \mathrm{kV}$ because power supplies and high voltage components such as cables and connectors are not commercially available and fabricating high voltage components would significantly increase costs. Once the maximum electrode voltage is determined, the neutron intensity increases linearly with current and beam power. Though the maximum extracted beam current from the deuterium source is approximately $27 \mathrm{~A}$, the size and cost of the 
power supply as well as the cost of electricity becomes excessive beyond $400 \mathrm{~kW}$, which corresponds to a $2 \mathrm{~A}$ at $200 \mathrm{KeV}$.

\section{D-D Neutron Yield vs. Beam Energy}

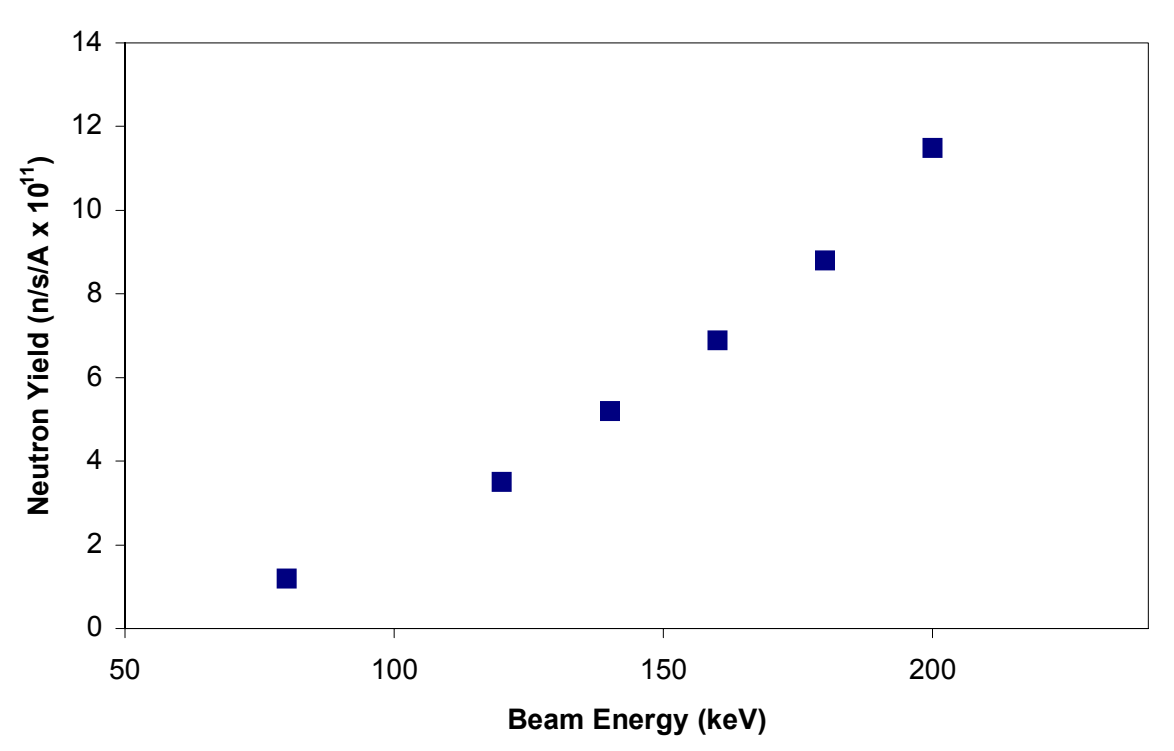

Figure 3 D-D neutron yield vs. beam energy

\section{Target Design}

The target consists of an inner diameter titanium layer on the order of 1 micron thick, a layer of copper to conduct the heat away from the target, a cooling channel for water and a coil (or permanent magnet set), and an outer layer of stainless steel. The permanent magnets or the coil is used to provide a small magnetic field to keep electrons from being accelerated towards the plasma electrode after the deuterons impact the target surface. The deuteron beam is used both to load the target and to drive the D-D reaction. The target is loaded continuously with the beam, and therefore has a long lifetime. The target lifetime is limited by the sputtering of the titanium layer. This layer thickness can be increased for longer life, but at the expense of decreased thermal conductivity. It is necessary to keep the target temperature between 100 and 200 degrees $\mathrm{C}$ so that the deuterium remains at the surface and does not migrate below the target surface. The heat generated in the target is approximately $200 \mathrm{~W} / \mathrm{cm}^{2}$.

\section{Reflector Design}

A reflector assembly is needed to direct as many of the source neutrons as possible to the SCM. For the purposes of this study, this source can be approximated by a monoenergetic isotropic source of neutrons. In choosing the material and dimensions of the reflector, a simple test geometry was to evaluate the relative reflectivity, leakage, and absorption of various materials. The thicknesses of these materials were varied from 20 to $60 \mathrm{~cm}$ and the number of reflected neutrons was counted as were the neutrons penetrating through the test material. This source and geometry were simulated in Monte Carlo N-Particle Transport code (MCNP). The geometry was 
a $10 \mathrm{~cm}$ diameter cylinder of reflective material with one end being a unidirectional source of 2.5 $\mathrm{MeV}$ neutrons. On each end of the cylinder, there were volumes with tally surfaces, which counted the surface current, or number of source neutrons going through the surfaces. The sides of the cylinder were made to be perfectly reflective to simulate an infinite slab.

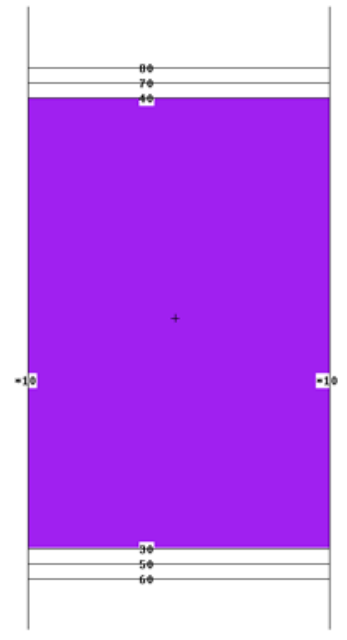

Figure 4 MCNP model for reflector study.

Water, heavy water, lead, and graphite were evaluated as reflector materials. $\mathrm{D}_{2} \mathrm{O}$ and lead were very similar and performed much better than the graphite. The water performed the most poorly as a result of the high absorption cross section due to the hydrogen. These tests were done with ten thousand particles and had errors between $1 \%$ and $5 \%$.

\section{Reflectivity}

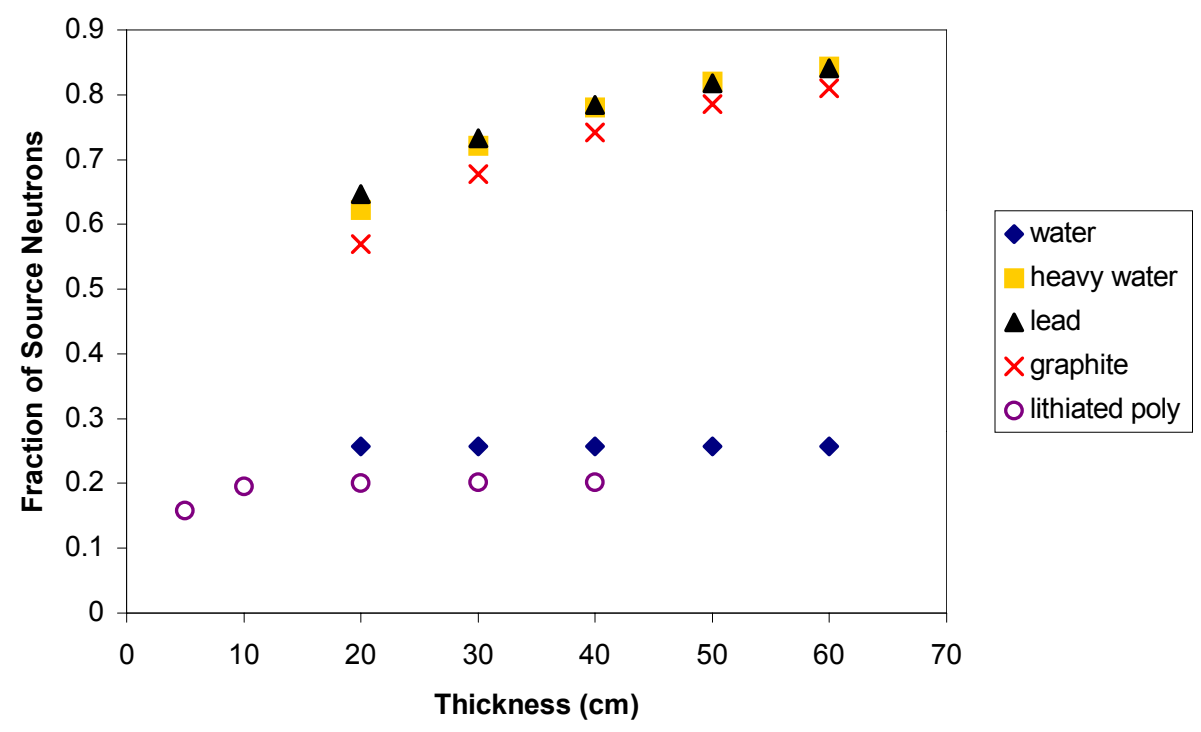

Figure 5 Reflectivity data. 


\section{Leakage}

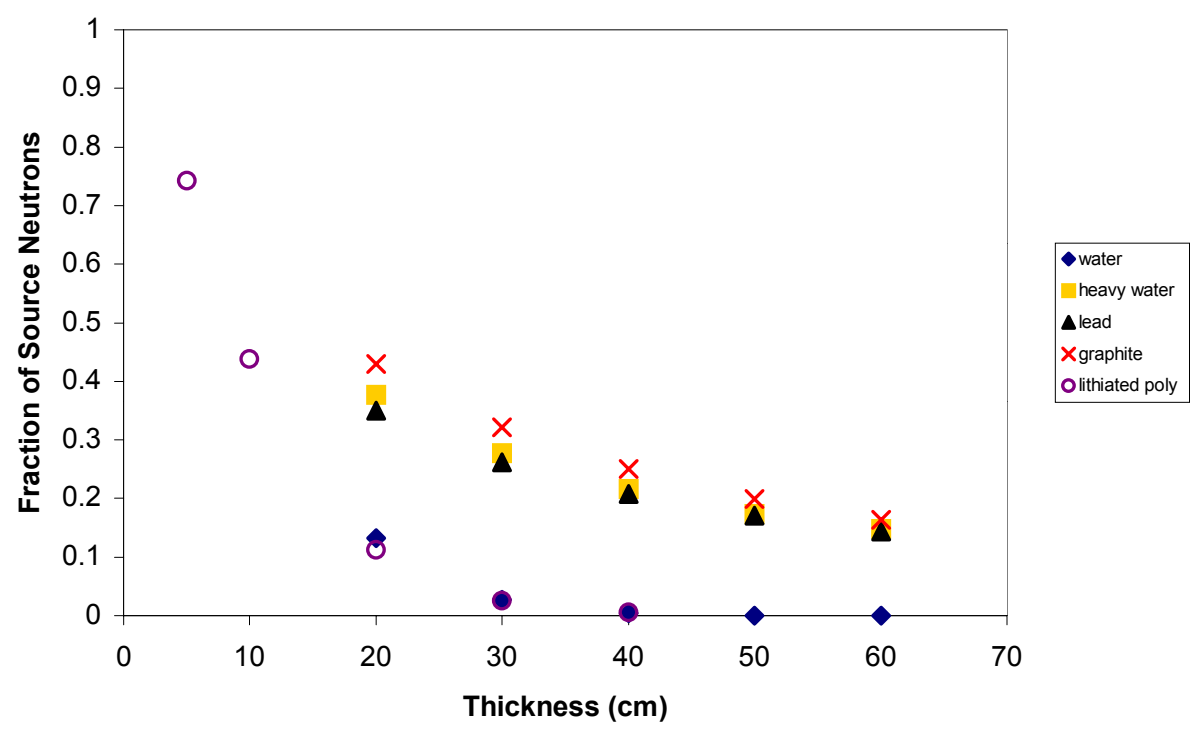

Figure 6 Leakage data

With the modeling of $30 \mathrm{~cm}$ of lead, heavy water, and graphite, it can be seen that the lead maintains a fairly hard spectrum at $2.5 \mathrm{MeV}$ with very few low energy neutrons. Compared to the lead, the $\mathrm{D}_{2} \mathrm{O}$ decreases the $2.5 \mathrm{MeV}$ peak by half and produces a concentration of thermal neutrons. The graphite is similar to the $\mathrm{D}_{2} \mathrm{O}$ but the changes from the lead spectrum are not as extreme. One can conclude that if the efficiency of the SCM is heavily dependent on the higher cross section for fission for the lower energy neutrons, it may be advantageous to use $\mathrm{D}_{2} \mathrm{O}$ as the reflector and add gamma-ray shielding closer to the outside of the assembly.

Using this data, a reflector assembly was designed to reflect source neutrons to the SCM. The main reflector consists of a half cylinder surrounding the cylindrical source with blocks forming the interface between the source and the SCM. The reflector aperture was increased to increase the solid angle "seen" by the SCM (see Figure 7).
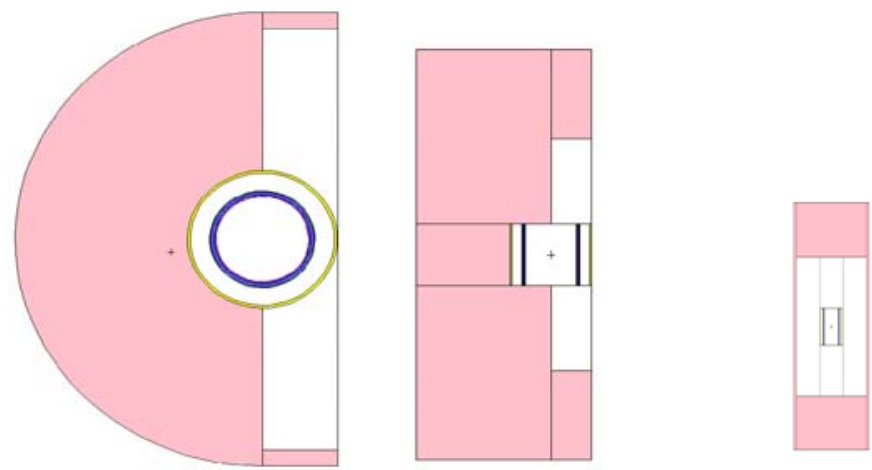

Figure 7 MCNP model views of source and reflector assembly (top, side, front). 
A tally plane was established at the interface between the source and SCM to determine the fraction and spectrum of neutrons passing into the SCM. As seen in Figure 8, about $40 \%$ of the source neutrons passed through the aperture with $30 \mathrm{~cm}$ of lead reflector. Heavy water, while reflecting a similar fraction of neutrons, was eliminated as a candidate since it moderated neutrons and decreased the $2.45 \mathrm{MeV}$ intensity, which may lead to greater backscatter from the SCM.

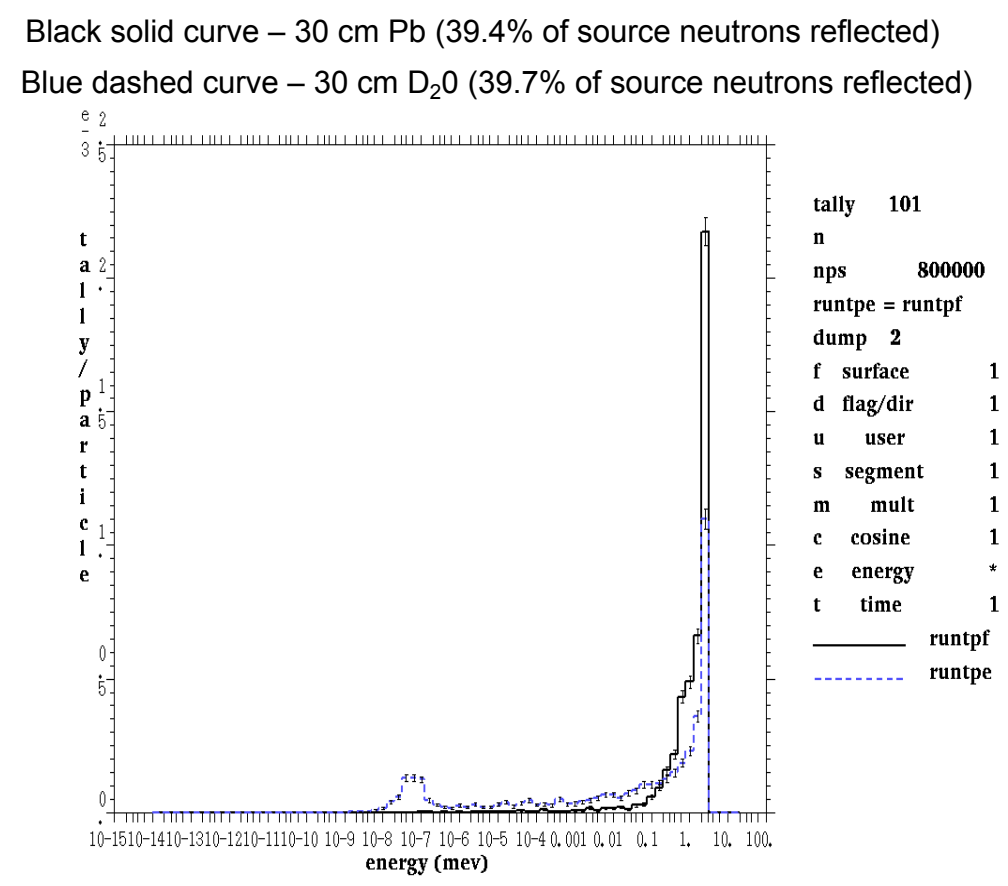

Figure 8 Output spectrum of lead and $\mathrm{D}_{2} \mathrm{O}$ reflectors.

\section{Shielding Design}

The same geometry used in the initial reflector study was used to investigate water and lithiated polyethylene as shielding materials to absorb low energy neutrons at the exit of the assembly. Though these materials had very similar absorbing properties, the lithiated polyethylene was chosen because hydrogen efficiently thermalizes the neutrons and the lithium absorbs the low energy neutrons without producing the capture gamma-rays of neutron absorption in hydrogen. Lithiated polyethylene is also easier to work with since it is a solid (see Figure 9). 


\section{Leakage}

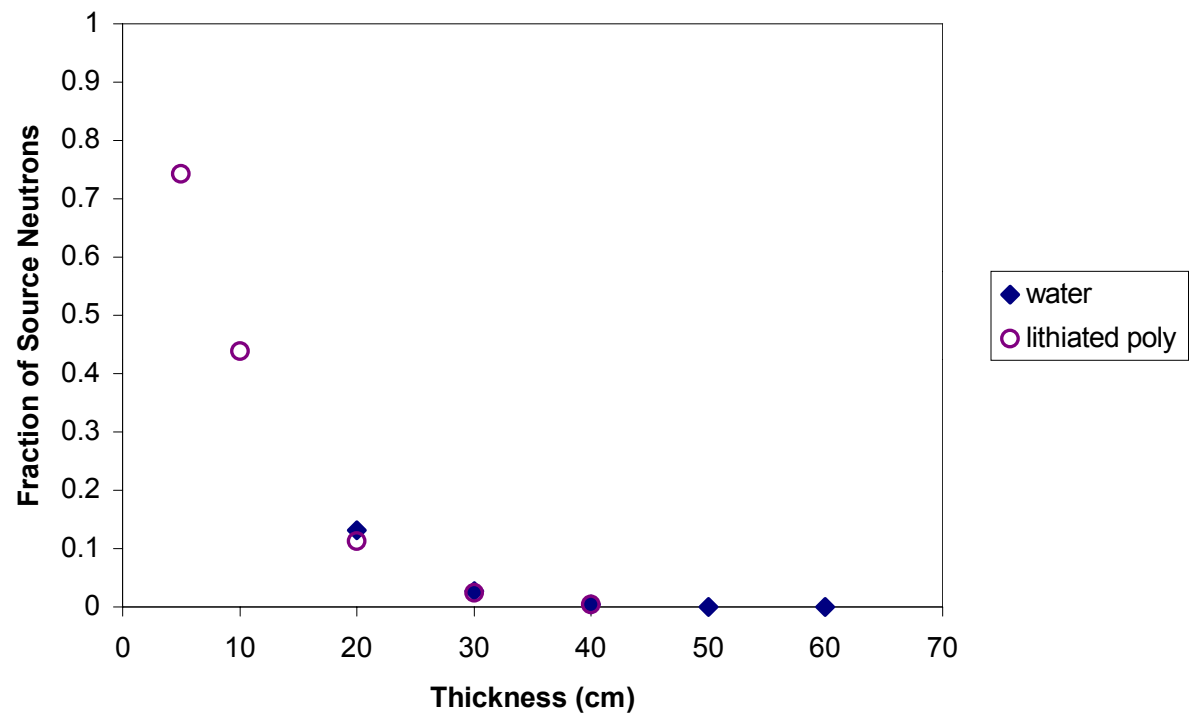

Figure 9 Leakage data for lithiated polyethylene and water.

\section{CNS Cooling}

The primary requirements for the cooling of the compact neutron source are to remove the beam power incident on the target and to maintain the target temperature between 373 and $473 \mathrm{~K}$ to control the diffusion of Deuterium in the target. While a passive cooling system is desired, the relatively high heat flux and confined geometry precludes the design of a cooling system based on passive processes like free convection. Fortunately, the CNS does not pose a significant risk in an accident scenario as the fusion plasma and the resulting beam power would dissipate rapidly in the event of an emergency. An automatic control system could be designed to quickly shutdown the power to the source when tripped by an alarm from a set of redundant thermocouples imbedded in the system. If this system fails, a quartz pressure vessel provides additional containment for items that have melted and vaporized coolant water could be vented to the atmosphere. Furthermore, replacing damaged portions of the source are fairly straightforward and inexpensive. As a result, a pump driven forced flow system is sufficient to remove the beam power and ensure safety. However, a set of redundant pumps may be desired to maximize the availability of the facility in the event maintenance on the pumps.

\section{Thermal Modeling}

For the thermal analysis, the components of interest are the copper target and the annulus of cooling water. The titanium layer on the inner surface of the copper target will be neglected as this micrometer thick layer is insignificant in comparison to the thickness of other materials. The stainless steel annulus forming the cooling channel will be treated as an insulated, adiabatic surface given the vacuum between the steel and the outermost quartz chamber. 


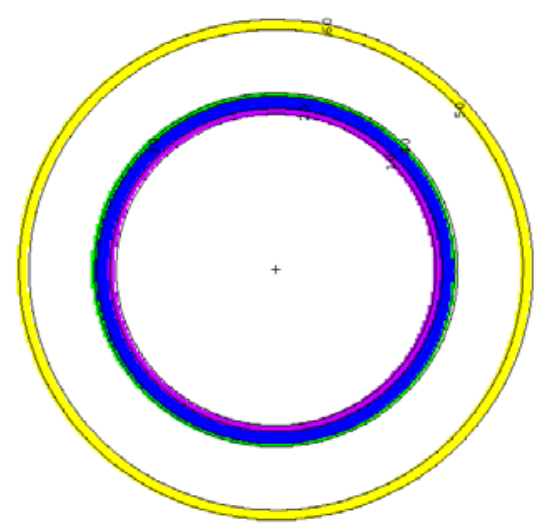

Figure 10 Cross section of the compact neutron source

Figure 10 shows a cross section of the compact neutron source as modeled in MCNP. The inner purple annulus is the copper target, the second blue annulus is the water layer, the third green layer is made of stainless steel, and the yellow annulus is a quartz chamber. The dimensions of interest are:

$r_{l}=0.0809 \mathrm{~m}$ (Inner radius of $\mathrm{Cu}$ target)

$r_{2}=0.0841 \mathrm{~m}$ (Outer radius of $\mathrm{Cu}$ Target and inner radius of water annulus)

$r_{3}=0.0905 \mathrm{~m}$ (Outer radius of water annulus)

$L=0.21 \mathrm{~m}$ (Length of cylindrical source)

\section{Power}

The compact neutron source is currently designed to extract a $1 \mathrm{~A}$ beam of Deuterium-Deuterium fusion plasma with an extraction voltage of $200 \mathrm{keV}$, resulting in a total beam power of $200 \mathrm{~kW}$ deposited on the target. Given the dimensions of the source, this beam power translates into a heat flux of approximately $188 \mathrm{~W} / \mathrm{cm}^{2}$ at both the inner and outer surfaces of the copper target that must be transferred to the cooling water. The designers of the source claim that up to $600 \mathrm{~W} / \mathrm{cm}^{2}$ can be easily removed using water. ${ }^{\text {vii }}$

\section{Steady State, Fully Developed, Forced Turbulent Convection in a Smooth Annulus}

The heat flux crossing the outer surface of the target to the cooling water can be determined by Newton's law of cooling,

$q^{\prime \prime}{ }_{i}=h_{i}\left(T_{2}-T_{\infty}\right)$

where $q$ " is the heat flux in $\mathrm{W} / \mathrm{m}^{2}, h$ is the convection heat transfer coefficient in $\mathrm{W} /\left(\mathrm{m}^{2} \mathrm{~K}\right)$, and $\left(T_{2}-T_{\infty}\right)$ is the temperature difference in Kelvin between the outer surface of the target and the coolant.

Determining the value of the convection heat transfer coefficient, $h$, requires knowledge of the Nusselt number $(\mathrm{Nu})$ defined as,

$$
N u_{i}=\frac{h_{i} D_{h}}{k_{f}}
$$


where $k_{f}$ is the conductivity of the cooling fluid in $\mathrm{W} /(\mathrm{mK})$ and the hydraulic diameter, $D_{h}$, in meters defined as,

$D_{h}=D_{3}-D_{2}=2\left(r_{3}-r_{2}\right)$

The Nusselt numbers for forced turbulent convection at the inner surface of a smooth concentric annular duct in the fully developed limit can be determined with a specified uniform heat flux, $q{ }^{\prime}$, at the inner surface and an insulated outer surface $\left(q{ }^{\prime}=0\right)$ by the following correlation,

$N u_{i}=\frac{h_{i} D_{h}}{k_{f}}=\frac{N u_{i i}}{1-\frac{q_{0}{ }_{0}}{q_{i}^{\prime \prime}} \theta_{i}}=N u_{i i}$

where the influence coefficient, $\theta_{i}$, is eliminated by the zero heat flux at the outer boundary. ${ }^{\text {ix }}$

Kays and Leung determined the Nusselt number $N u_{i i}$ and influence coefficients as a function of the Reynold's number $(R e){ }^{\mathrm{x}}$

$\operatorname{Re}=\frac{u D_{h}}{v}$

where $u$ is the fluid velocity in $\mathrm{m} / \mathrm{s}, D_{h}$ is the hydraulic diameter in $\mathrm{m}$, and $v$ is the kinematic viscosity in $\mathrm{m}^{2} / \mathrm{s}$; the Prandtl number $(\mathrm{Pr})$,

$\operatorname{Pr}=\frac{v}{\alpha}=5.82$ (for water at $300 \mathrm{~K}$ )

where $v$ is the kinematic viscosity in $\mathrm{m}^{2} / \mathrm{s}$ and $\alpha$ is the thermal diffusivity in $\mathrm{m}^{2} / \mathrm{s}$; and the ratio between the inner and outer radii of the annulus $\left(r^{*}\right)$,

$r^{*}=\frac{r_{3}}{r_{2}}$

Unfortunately, Kays and Leung do not provide a Nusselt number for the compact neutron source geometry $\left(r^{*}=0.929\right)$. However, the Nusselt number reaches a limiting value as $r^{*}$ approaches unity for a Reynolds's number of $3 \times 10^{4}$ and a Prandtl number of 5.82 (see Figure 11). Without a tabulated value, this analysis will assume a Nusselt number corresponding to an $r^{*}$ of 0.8 , which is higher than the apparent limiting value. This approximation will tend to overestimate heat transfer since the convective heat transfer coefficient is proportional to the Nusselt number. Examining Figure 11, the error in the estimate of the Nusselt number is on the order of \pm 10 . 


\section{$\mathrm{Nu}_{\mathrm{ii}}$ vs. $\mathrm{r}^{*}$ \\ $\mathrm{Re}=3 \mathrm{E} 4, \operatorname{Pr}=5.82$}

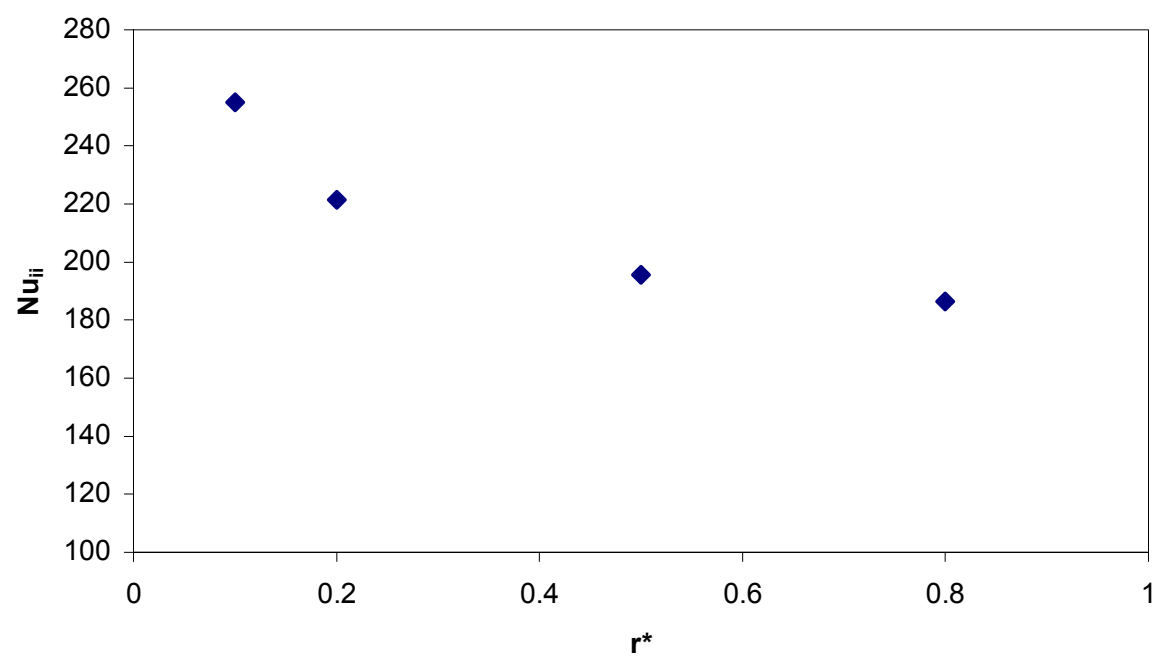

Figure $11 \mathrm{Nu}_{\mathrm{ii}}$ vs. $\mathrm{r}^{*}$ for $\mathrm{Re}=3 \times 10^{4}, \mathrm{Pr}=5.82$ fluid.

Based on the above analysis, the convective heat transfer coefficient, $h$, was found to be about $12200 \mathrm{~W} /\left(\mathrm{m}^{2} \mathrm{~K}\right)$ for a heat flux of about $188 \mathrm{~W} / \mathrm{cm}^{2}$ and a cooling water temperature of $300 \mathrm{~K}$. Solving Newton's law of cooling for the temperature of the outer surface of the target,

$T_{2}=T_{\infty}+\frac{q^{\prime \prime}}{h}=300 \mathrm{~K}+\frac{188 \frac{\mathrm{W}}{\mathrm{cm}^{2}}}{12200 \frac{\mathrm{W}}{\mathrm{m}^{2} \mathrm{~K}}}=448.9 \pm 0.1 \mathrm{~K}$

where the approximate error is given by, $\sigma_{T_{2}}=\sqrt{\left(\frac{\sigma_{q^{\prime \prime}}}{h}\right)^{2}+\left(\frac{-q^{\prime \prime} \sigma_{h}}{h^{2}}\right)^{2}}=\frac{q^{\prime \prime}}{h^{2}} \sigma_{h}=0.1 K$

assuming that the error in the heat flux is negligible and where $\sigma_{x}$ is the error in variable $x$.

\section{Radial Conduction}

Using the temperature of the outer target surface, the radial temperature profile of the copper annulus can be calculated by assuming that the conductivity of copper remains constant. The temperature profile is given by,

$T(r)=\frac{T_{1}-T_{2}}{\ln \frac{r_{1}}{r_{2}}} \ln \frac{r_{1}}{r_{2}}+T_{2}$ for $r_{1}<r<r_{2}$

and the difference in surface temperatures is given by,

$$
T_{1}=T_{2}+\frac{\dot{q} \ln \frac{r_{2}}{r_{1}}}{2 \pi L k_{C u}}
$$


where the subscript 1 corresponds to the inner surface, the subscript 2 corresponds to the outer surface of the copper annulus, $T$ is the temperature in $\mathrm{K}, r$ is the radius in meters, $\dot{q}$ is the beam power in $\mathrm{W}$, and $L$ is the length of the source in meters.

Performing this analysis for the target, the maximum target temperature is approximately $463 \mathrm{~K}$, about $10 \mathrm{~K}$ under the maximum desired operating temperature of $473 \mathrm{~K}$. The radial temperature profile of the target is plotted in Figure 12.

\section{Radial Temperature Profile of Copper Target}

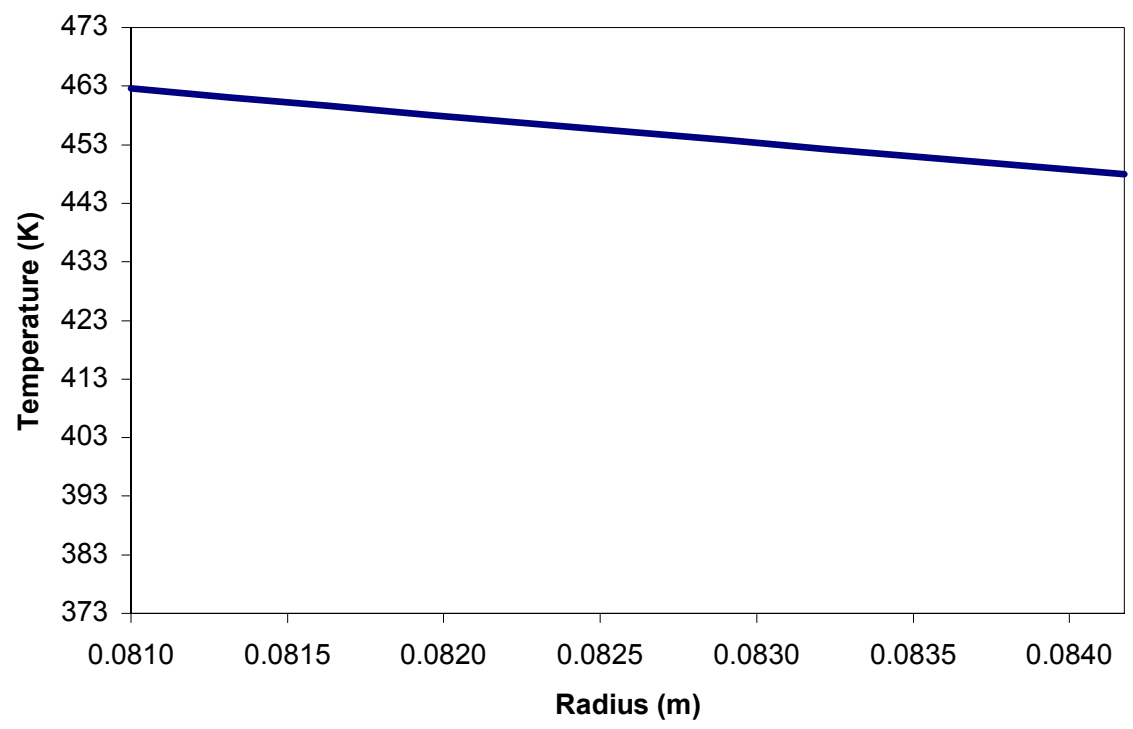

Figure 12 Radial temperature profile of the copper target.

\section{Fluid Flow}

The flow velocity can be easily determined from the Reynolds's number $\left(R e=3 X 10^{4}\right)$ as follows, $u=\frac{\operatorname{Re} \times v}{D_{h}}=2.03 \mathrm{~m} / \mathrm{s}$

The volumetric flow rate can then be determined based on the annular area, $\dot{V}=u A=u \pi\left(r_{o}^{2}-r_{i}^{2}\right)=0.007 \mathrm{~m}^{3} / \mathrm{s}=1.86^{\mathrm{gal}} / \mathrm{min}$ and the mass flow rate can be determined with the density of the fluid at $300 \mathrm{~K}$, $\dot{m}=\rho \dot{V}=7 \mathrm{~kg} / \mathrm{s}$

Such flowrates are achievable with typical commercially available pumps and should not present major difficulties.

\section{Conclusion}

This neutron source may potentially provide a compact, simple, safe, and effective design compared to D-T sources and other accelerator-based systems. This same neutron source is currently being tested at LBNL and there will be operational experience and neutron yield data 
within the next year. In the case of insufficient source intensity either in the simulations or in testing, the beam current can be increased for a linear increase in neutron yield. This additional power must be supplied by the power supply and dissipated in the target. The reflector has been optimized for the highest efficiency in coupling neutrons to the SCM, and the integrated design will be modified after reliable results are attained from the safety analysis and the patient dose calculations.

While cooling the source is fairly straightforward, the source must be shielded to protect patients and operators from high neutron doses. Difficulties arise when routing ducts through the shielding material as any gap in the shielding provides a path for neutron diffusion. Such effects can be minimized through the design of labyrinthine paths for water flow, though additional pumping power would be required to overcome frictional losses in a convoluted piping system.

\section{SUBCRITICAL FISSION MULTIPLIER (SCM)}

\section{Introduction \& Methods}

As mentioned earlier, a D-D fusion based CNS does not have sufficient intensity to achieve acceptable treatment times. Utilizing a subcritical fission multiplier to increase neutron intensity has two main advantages. The first is to significantly reduce the amount of time a patient is exposed to an epithermal neutron flux without the use of tritium (in D-T fusion neutron sources). Using D-D fusion neutrons is advantageous because the system used to create the neutrons is inherently safe, whereas radioactivity of tritium gas complicates handling. The second advantage is that of cost and efficiency. Ideally, a subcritical system can multiply the number of source neutrons by a factor of 50 .

MCNP was used extensively in the iterative design of the SCM for criticality calculations and the neutron fluxes and currents. MCNP simulates the behavior of neutrons and other energetic subatomic particles in their reactions with various materials, including fissile $\mathrm{UO}_{2}$ fuel. Specifying a particular number of source particles, MCNP can run a sufficient number of trials to measure the $\mathrm{k}_{\mathrm{eff}}$ of the system. The theoretical subcritical multiplication factor can be determined from $\mathrm{k}_{\mathrm{eff}}$ as follows,

$$
\mathrm{SCM}=1 / 1-\mathrm{k}_{\text {eff }}
$$

\section{Design Process \& Goals}

The ultimate goal of the SCM is to maximize the multiplication of source neutrons without achieving criticality and with fuel enriched to less than $20 \mathrm{w} / \mathrm{o}{ }^{235} \mathrm{U}$ to minimize proliferation concerns. Geometrical concerns are vitally important as the leakage of neutrons from undesirable surfaces must be minimized and a large fraction of source neutrons should be absorbed in the SCM all while maximizing the neutron intensity output from the SCM. Realistically, an array of $\mathrm{UO}_{2}$ fuel pins similar to those found in reactors would likely be used. However, for modeling and analytical simplicity, the investigation focused on a series of low aspect ratio cylindrical plates with light water moderator/coolant layers. 


\section{Design Variables}

Once the basic geometry was established, it was necessary to specify the physical dimensions of the system as well as the properties of the materials used in its fabrication. The physical dimensions of the cylindrical SCM include the fuel-region diameter, length, number of plates, $\mathrm{k}_{\text {eff }}$ (and thus the subcritical multiplication factor), fuel enrichment, moderator-to-fuel ratio, and neutron backscattering due to the coupling of the SCM to the source and the Beam Shaping Assembly.

\section{Material Properties}

The fuel of choice is standard enriched $\mathrm{UO}_{2}$, similar to that in nuclear power reactors. In the MCNP model, a $1 \mathrm{~mm}$ thick Zircaloy-4 cladding with a helium gap is used. The cladding properties and composition are shown below:

Table 1 Zircaly-4 Properties

\begin{tabular}{|l|l|l|}
\hline \multicolumn{3}{|l|}{ Zircaloy-4 Cladding Properties } \\
\hline Density & $6.44 \mathrm{~g} / \mathrm{cm}^{3}$ & \\
\hline Composition & $\mathrm{Zr}$ & 97.91 wt. \% \\
\hline & $\mathrm{Sn}$ & 1.59 wt. \% \\
\hline & $\mathrm{Fe}$ & 0.5 wt. $\%$ \\
\hline
\end{tabular}

Other materials used in the MCNP analysis include light water to be used as a moderator and reflector, lead for energy-spectrum hardening and gamma-ray shielding, and lithiated polyethylene to thermalize and absorb neutrons. Thick layers of aluminum and lead, the predominant materials in the Beam Shaping Assembly and the neutron source, respectively, was used to simulate backscattering at the interfaces between the sections.

\section{Plate Diameter}

The diameter of the plates was fixed at $80 \mathrm{~cm}$. According to Verbeke, the full-width-half-max (FWHM) of the epithermal peak of his BSA's output spectrum was minimized with an $80 \mathrm{~cm}$ diameter coupling between his source and BSA. ${ }^{\mathrm{xi}}$ Also, larger diameter plates are advantageous since they intercept the source neutrons over a larger solid angle.

Manufacturing large diameter $\mathrm{UO}_{2}$ plates and cladding seems mechanically impractical. Additionally, given the unique geometry of the SCM, the fabrication cost would undoubtedly be high. As mentioned earlier, the most practical alternative to the chosen geometry could utilize a lattice of enriched uranium fuel pins to better emulate a conventional nuclear reactor.

\section{Moderator/Fuel Ratio}

Light water was chosen for moderation and cooling purposes due to its abundance, minimal cost, and excellent moderation properties. (The thermal diffusion length of neutrons in water is 2.85 $\mathrm{cm}$.) The optimum water/fuel ratio was determined empirically using a Monte Carlo k-code 
calculation. To simulate the environment of a fuel plate far from the ends of the cylinder, a water "sandwich" was modeled with $\mathrm{UO}_{2}$ meat. A 1-cm thick infinite slab of $\mathrm{UO}_{2}$ was modeled with varying thicknesses of water on each side of the plate.

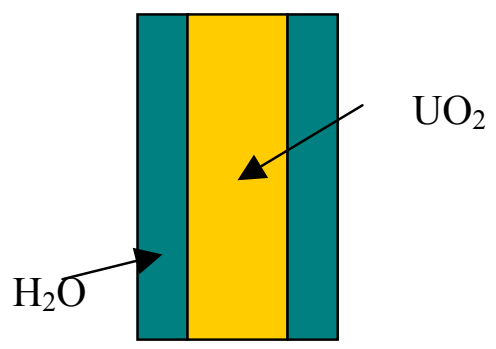

Figure 13 Infinite slab model

The following chart shows a range of values for water-to-fuel ratio and the corresponding value of $k_{\text {inf }}$ measured using MCNP's k-code. As seen graphically in Figure 15, a maximum occurs when the volumetric water-to-fuel ratio is approximately $2.25 / 1$. The maximum $\mathrm{k}_{\text {inf }}$ is also nearly independent of the fuel enrichment over the range of $5-20 \mathrm{w} / \mathrm{o}{ }^{235} \mathrm{U}$. The ratio of $2.25 / 1$ was selected for further analysis.

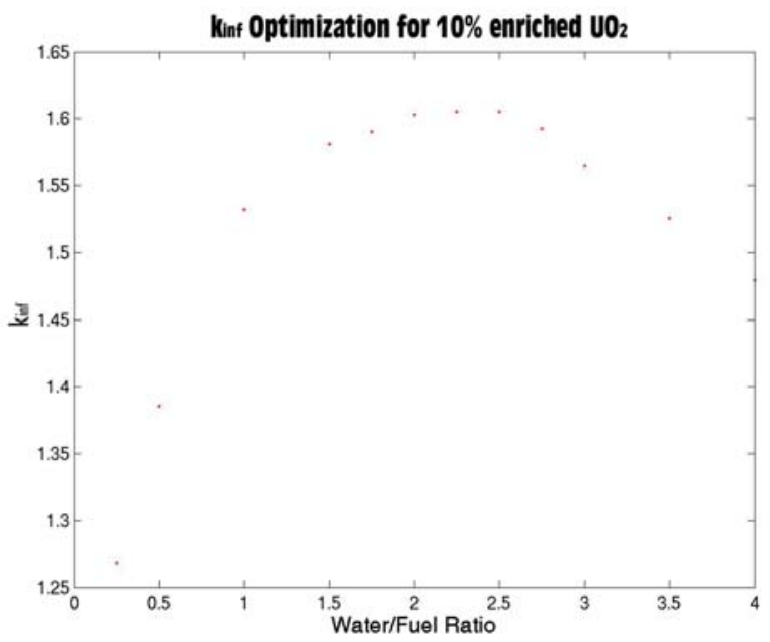

Figure $14 \mathrm{k}_{\text {inf }}$ vs. water-fuel volume ratio

\section{Length Optimization}

An iterative design approach was taken to determine the optimum number of heterogeneous fuel plates that would minimize radial leakage, maximize the absorption of source neutrons in the fuel, and most importantly maximize neutron multiplication. $\mathrm{k}_{\text {eff }}$ was kept close to 0.98 (the upper limit determined to be safe for a subcritical system) to maximize neutron multiplication. 


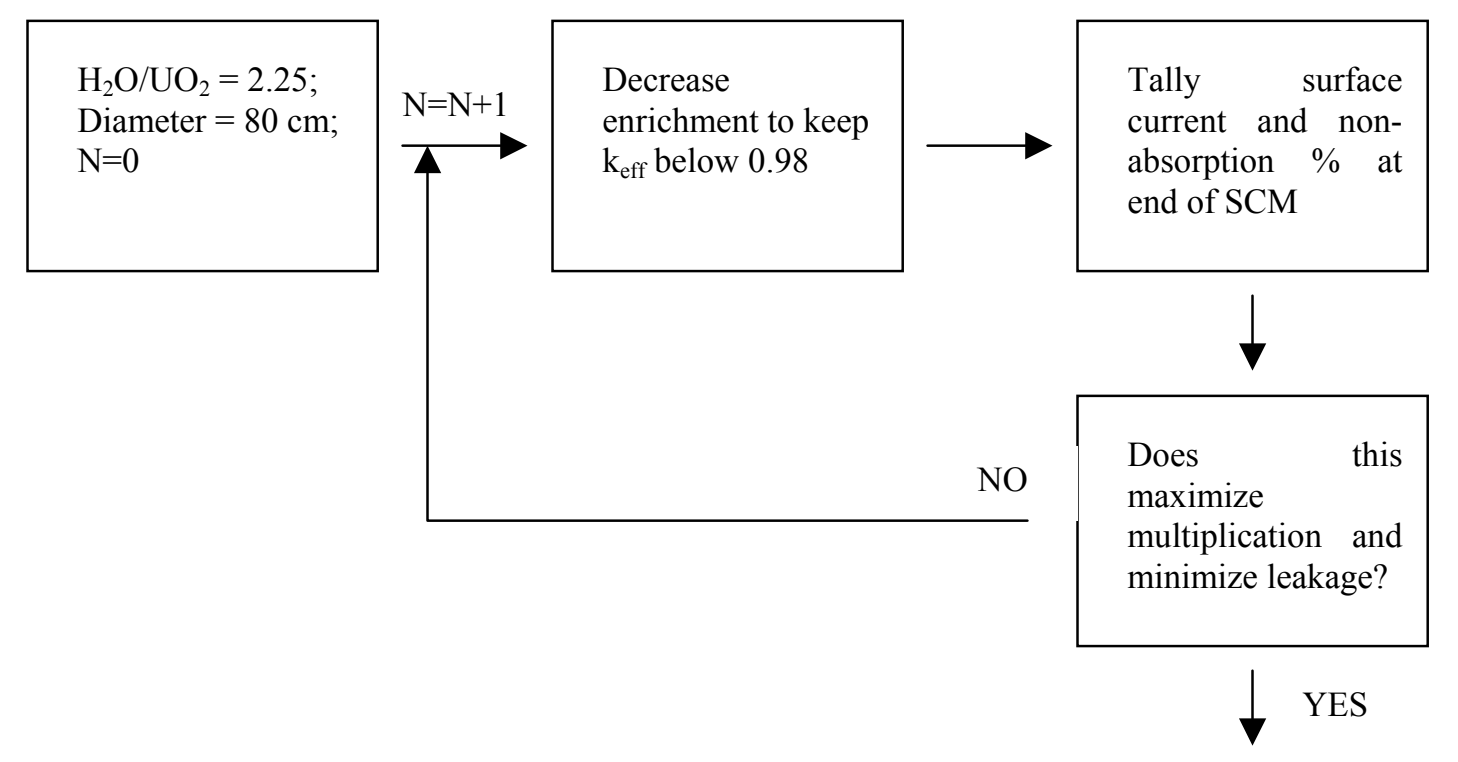

$\mathrm{N}_{\text {final }}=$ optimum number of plates

Figure 15 Flowchart showing iterative design process.

\section{Multiplication and Leakage}

To quantify the radial neutron leakage from the SCM cylinder, a tally surface was created around the outer annulus of lead and at the circular ends of the cylindrical volume. The source was modeled as a monodirectional, monoenergetic $(2.5 \mathrm{MeV})$ planar source, $16 \mathrm{~cm}$ in diameter. The source was placed nearly against the first fuel plate to model the source and inside the tally surface. The desired neutron leakage is out of the right circular surface in an axial direction.

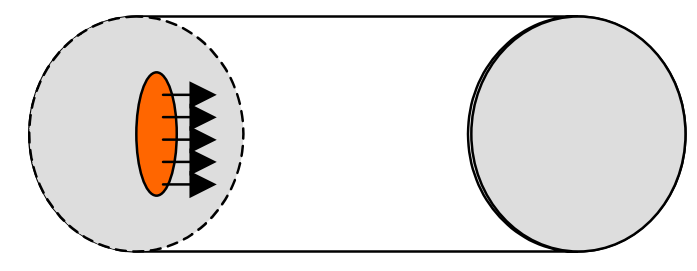

Figure 16 Tally surfaces

Measurements of the current leaking from this surface showed a peak in the subcritical multiplication factor at a length corresponding to five plates as shown in Table 2. 
Table 2 Multiplication

\begin{tabular}{|l|l|}
\hline Number of Plates & $\begin{array}{l}\text { Surface Current (per } \\
\text { source particle) }\end{array}$ \\
\hline 2 & 9.0 \\
\hline 4 & 27.9 \\
\hline 5 & 30.4 \\
\hline 6 & 26.8 \\
\hline
\end{tabular}

\section{Absorption}

To quantify the number of neutrons that are not absorbed in the fissile material and instead pass through the system to the output tally surface (as a function of length), the MCNP nonu, fission turnoff card was used. Using this card, MCNP simply turns the fission off, treating fission as an absorption event without the production of fission neutrons. As a result, the fraction of nonabsorbed source neutrons can be determined. As expected, a longer SCM will absorb more source neutrons and produce more fission events. Unfortunately, increasing the length of the SCM increases radial leakage, the net effect of which is a reduction in the observed neutron multiplication. For the five-plate geometry described above, $45.1 \%$ of the neutrons were absorbed in the fuel region while $54.9 \%$ leaked from the outer surfaces.

\section{Moderation/Reflection Study}

In order to improve the absorption efficiency of source neutrons, one possible solution is to premoderate the $2.5 \mathrm{MeV}$ source neutrons in a water layer before they enter the first uranium dioxide plate. However, neutrons are lost to reflection from the water layer. The MCNP model incorporated a 5-cm thick cylindrical volume of water between the source and SCM. Results showed that the net current flowed in the direction of the -1 to 0 cosine bin, corresponding to the direction of the source. Therefore, the pre-moderation was deemed unworthy of further investigation as it represented an unacceptable loss of source intensity.

\section{Integration, Enrichment, and $k_{\text {eff }}$}

The final geometry of the SCM module to be inserted into the integrated design consisted of five $\mathrm{UO}_{2}$ plates of $3.8 \%$ enrichment with a $\mathrm{k}_{\text {eff }}$ of 0.983 . Zircaloy cladding surrounds the uranium fuel plates and a $30-\mathrm{cm}$ thick annulus of lead is used around the SCM as a reflector and shield. Covering the lead is a $20-\mathrm{cm}$ thick annulus of Lithiated polyethylene to thermalize and absorb neutrons.

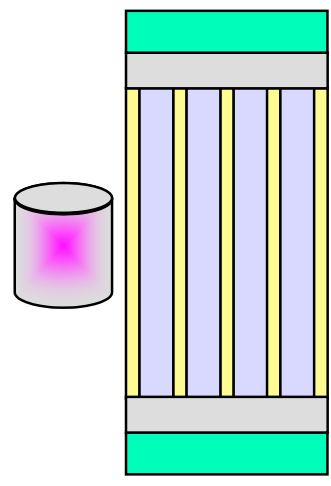

Figure 17 Diagram of CNS-to-SCM coupling 


\section{Burnup}

A rough estimate of fuel burnup and SCM lifetime can be determined based on SCM power and fuel geometry. As it will be shown in the thermal analysis, SCM power will not exceed $1 \mathrm{~kW}$. Given the geometry of the SCM, the combined volume of the five $\mathrm{UO}_{2}$ plates is about 25,000 $\mathrm{cm}^{3}$. Assuming the density of $3.8 \%$ enriched UO2 is $10.93 \mathrm{gm} / \mathrm{cm}^{3}$ at $300 \mathrm{~K}$, the mass of uranium dioxide is about 0.27 metric tons $(\mathrm{Mt})$ or approximately $0.24 \mathrm{Mt}$ of Uranium. Assuming 24 months of operation at constant maximum power of $1 \mathrm{~kW}$, the fuel burnup is estimated at about 3MWD/MTU as follows:

Total Burnup $=730$ days $\mathrm{x} 1 \mathrm{~kW}=730 \mathrm{kWd}$

Specific Burnup $=$ Total burnup / mass of uranium $=730 \mathrm{kWd} /(0.2747 \times[238 /\{2 \times 16+$ $238\}])=3 \mathrm{kWd} / \mathrm{t}$ (or $3 \mathrm{MWd} / \mathrm{MTU})$

In comparison, current light water reactor fuel typically have burnups around 30,000 MWd/MTU, indicating that the SCM would likely have a long lifetime before burnup effects, such as reactivity swings, have major effects on the system.

\section{SCM Cooling \& Safety}

In contrast to the source, cooling of the subcritical multiplier (SCM) has significant impacts on safety. The power generated by the source driven SCM must be effectively removed to ensure that all materials, especially the uranium oxide fuel and the Zircaloy cladding, do not melt or fail. With such considerations in mind, a passive heat removal system is desired to eliminate the possibility of overheating due to the failure of operators or the failure of active cooling systems.

\section{SCM Design}

The SCM consists of five slightly enriched $\mathrm{UO}_{2}$ cylinders $80 \mathrm{~cm}$ in diameter and $1 \mathrm{~cm}$ in thickness. Around the uranium plates is a $1 \mathrm{~mm}$ thick layer of helium gas to provide room for fuel swelling and gaseous fission products. The cladding is a $1 \mathrm{~mm}$ thick layer of Zircaloy. The fuel region is then surrounded by a $30 \mathrm{~cm}$ layer of lead to provide neutron reflection and photon shielding. A $2.25 \mathrm{~cm}$ layer of light water is used as a moderator and coolant between each fuel plate. Finally, the current design of the SCM has a layer of lithiated polyethylene surrounding the lead shield to moderate and absorbs neutrons escaping from the lead shielding. The melting temperatures of the various materials are:

- UO2: $3120 \mathrm{~K}$

- Zircaloy: $2123 \mathrm{~K}$

- Lead: $601 \mathrm{~K}$

- Lithiated Polyethylene: $410 \mathrm{~K}$

Unfortunately, given the relatively low melting temperature and low conductivity of polyethylene, this layer cannot be in contact with the SCM as it would probably melt. Instead, this analysis will model the SCM inside of a pool of coolant water with the final neutronabsorbing layer, an additional photon shield, and other structural materials forming a structure containing the coolant (see Error! Reference source not found.). Additionally, it will be assumed that there is a path for water flow through the lead shielding and between the fuel 
plates. The effect of these water ducts, the water pool, and the external shielding on the neutronics of the system has not been modeled at this point.

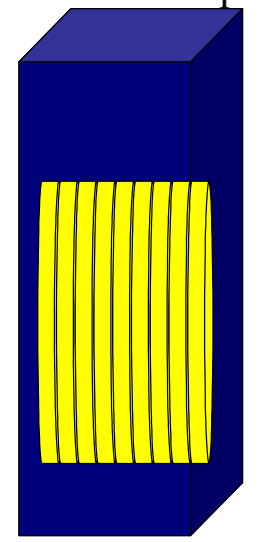

Figure 18 SCM in water pool.

\section{Power Estimate}

An estimate of the power produced by the source driven Subcritical Multiplier (SCM) can be determined as follows,

$$
\dot{q}=(n)\left(\frac{1}{1-k_{\text {eff }}}\right)\left(\frac{1 \text { fission }}{2.4 \text { neutrons }}\right)\left(\frac{200 \mathrm{MeV}}{\text { fission }}\right)=668 \mathrm{~W}
$$

where $n$ is the neutron source intensity $\left(1 \times 10^{12} \mathrm{n} / \mathrm{s}\right)$ and $k_{\text {eff }}$ is 0.98 . However, this estimate of SCM power is very conservative since over half of the source neutrons are lost to leakage and additional neutrons are lost to leakage in the multiplier. Actual multiplier power should be less than $333 \mathrm{~W}$. This analysis will conservatively estimate a peak total power of $1 \mathrm{~kW}$.

\section{Thermal Modeling}

A full thermal analysis of the SCM is complicated by the necessity of characterizing heat transfer in both the radial and axial directions. However, each axis corresponds to different operating conditions and so, this analysis can be simplified by considering each axis one at a time. Under normal operating conditions with sufficient coolant flow between fuel plates, the majority of the power would be dissipated between the faces of the fuel plates and the flowing water layers. In the case of a loss of coolant accident, the space between fuel plates would be insulated by an air gap. While some airflow may occur, axial centerline temperatures would rise such that the majority of the SCM power would be conducted radially to the surrounding medium.

\section{Steady State Axial Heat Transfer}

This analysis will assume steady state conduction and convection. For additional simplicity, the system will be approximated as rectangular isoflux plates equal in area to the circular plates with a height equal to the diameter of the circular plates. Radial heat transfer will be ignored.

Free Convection within Vertical Parallel Plate Channels 
Buoyancy forces can drive fluid flow between two heated vertical plates. To determine the heat transfer due to free convection between vertical isoflux plates, Bar-Cohen and Rohsenow define the Nusselt number as,

$$
N u_{S, L}=\left(\frac{q_{s}^{\prime \prime}}{T_{s, L}-T_{\infty}}\right) \frac{S}{k_{f}}
$$

where $N u_{S, L}$ is the local Nusselt number, $q{ }^{\prime}{ }_{s}$ is the heat flux, $T_{S, L}$ is the temperature at the top of the plate $(x=L)$ where the surface temperature is highest, $T_{\infty}$ is the temperature of the medium at the entrance of the duct, $S$ is the spacing between plates, and $k_{f}$ is the thermal conductivity of the fluid. The relative effects of buoyancy in comparison to viscous forces in the fluid is determined by the modified Rayleigh number, $R a_{S}$, defined as,

$$
R a_{S}^{*}=\frac{g \beta q_{s}^{\prime \prime} S^{4}}{k_{f} \alpha v}
$$

where $g$ is gravitational acceleration in $\mathrm{m} / \mathrm{s}^{2}, \beta$ is the coefficient of expansion in $\mathrm{K}^{-1}, \alpha$ is the thermal diffusivity in $\mathrm{m}^{2} / \mathrm{s}$, and $v$ is the kinematic viscosity in $\mathrm{m}^{2} / \mathrm{s}$. Bar-Cohen and Rohsenow offers the following Nusselt number correlation for symmetric isoflux plates in the fully developed limit, ${ }^{\text {xi }}$

$$
N u_{S, L}=\left[\frac{48}{R a_{S}^{*} \frac{S}{L}}+\frac{2.51}{\left(R a_{S}^{*} \frac{S}{L}\right)^{2 / 15}}\right]^{-1 / 2}
$$

These correlations use the properties of the fluid evaluated at the average temperature of the fluid. For isoflux surfaces, fluid temperature varies linearly with distance and so the mean temperature is simply,

$$
\bar{T}=\frac{T_{S, L}+T_{\infty}}{2}
$$

\section{Steady State Axial Conduction}

Steady state axial temperature profiles in the SCM can be determined by assuming that conductivity values do not vary significantly with temperature. For a plane wall with internal heat generation, the temperature profile is,

$T(x)=\frac{q^{\prime \prime \prime} L^{2}}{2 k_{\mathrm{UO}_{2}}}\left(1-\frac{x^{2}}{L^{2}}\right)+T_{\text {surface }}$

where $x$ is the distance from the centerline of the plate, $q^{\prime \prime}$ ' is the volumetric power in $\mathrm{W} / \mathrm{m}^{3}, L$ is the thickness of the plate, $k$ is the conductivity, and $T_{\text {surface }}$ is the temperature of surface at $x=L$. The temperature profile through a plane wall without internal heat generation is linear and given by,

$T(x)=\left(T_{2}-T_{1}\right) \frac{x}{L}+T_{1}$

where the subscript 1 corresponds to the inner wall and the subscript 2 corresponds to the outer wall, $L$ is the thickness of the wall, and $x$ is the axial position. The difference in wall temperatures is given by, 
$T_{1}=T_{2}+\frac{q^{\prime \prime} L}{k}$

where $q$ " is the heat flux on the faces of the fuel plates. Properly applying the previous three equations to the different materials provides the temperature profile in the axial direction.

\section{Computational Methods}

An iterative technique was developed to converge on the surface temperature of the SCM assembly and was implemented in Microsoft Excel. The heat flux that needs to be removed is set by the power and geometry of the SCM. An arbitrary, realistic value of $T_{S, L}$ is picked and the modified Rayleigh number is computed with material properties evaluated at the mean temperature. The Nusselt number correlation provided by Bar-Cohen and Rohsenow is then calculated using the modified Rayleigh number. Given this Nusselt number and $T_{S, L}$, the heat flux removed by free convection is calculated. If the calculated heat flux is greater than the heat flux generated by the SCM, $T_{S, L}$ is reduced and vice versa until the calculated and required heat fluxes converge.

Based on this maximum surface temperature, the maximum axial temperature profile of the assembly is generated. This also requires an iterative technique as the conductivity of the materials change with temperature. To provide a conservative estimate of peak temperature, the lowest conductivity value is selected. This is accomplished by first choosing reasonable conductivity values and then evaluating the resulting temperature profiles. Based on these profiles, conductivity values are reevaluated at all temperatures and the minimum is selected for the next iteration on temperature. These iterations continue until conductivity values converged. This algorithm is outlined in the following flowchart (See Figure 19).

The thermal conductivity of $95 \%$ dense $\mathrm{UO}_{2}$ fuel was determined with the following relation provided by the International Nuclear Safety Center at the Argonne National Laboratory,

$k(t)=\frac{100}{7.5408+17.692 t+3.6142 t^{2}}+\frac{6400}{t^{5 / 2}} e^{-16.35 / t}$ were $t$ is the temperature in Kelvin, $T$, divided by $1000 .^{\text {xiii }}$ The thermal conductivity of Zircaloy for temperatures between 300 and $1800 \mathrm{~K}$ is given by, $k(t)=12.767-5.4348 \times 10^{-4} T+8.9818 \times 10^{-6} T^{2}$ where $T$ is the temperature in Kelvin. ${ }^{\text {xiv }}$ Other values, such as the conductivity of Helium were adjusted manually as necessary.

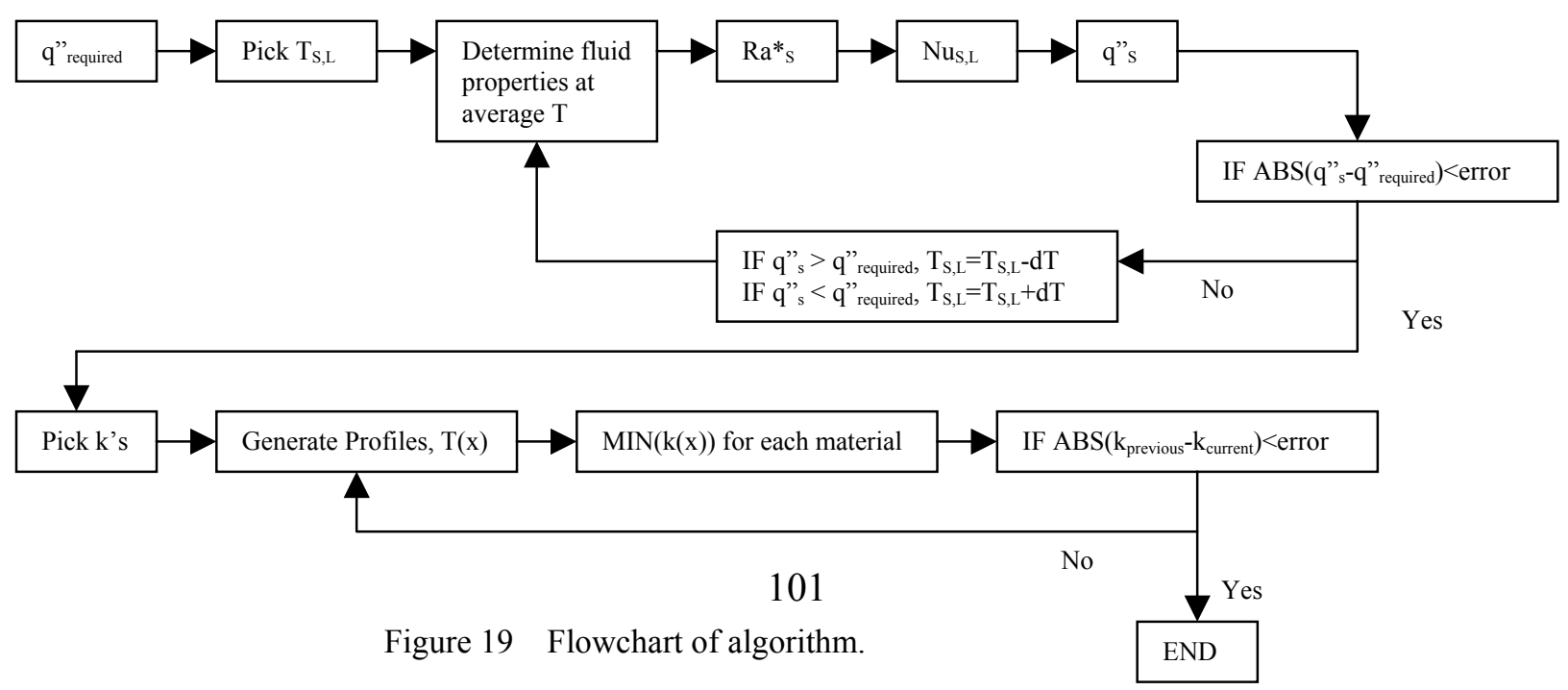




\section{Temperature Profiles}

This analysis was performed at three power levels, $1000 \mathrm{~W}, 100 \mathrm{~W}$, and $1 \mathrm{~W}$. Temperatures were well below the melting points of all materials at all of these power levels (see Figure 20).

\section{Free Convection Between Fuel Plates Axial Temperature Profiles}

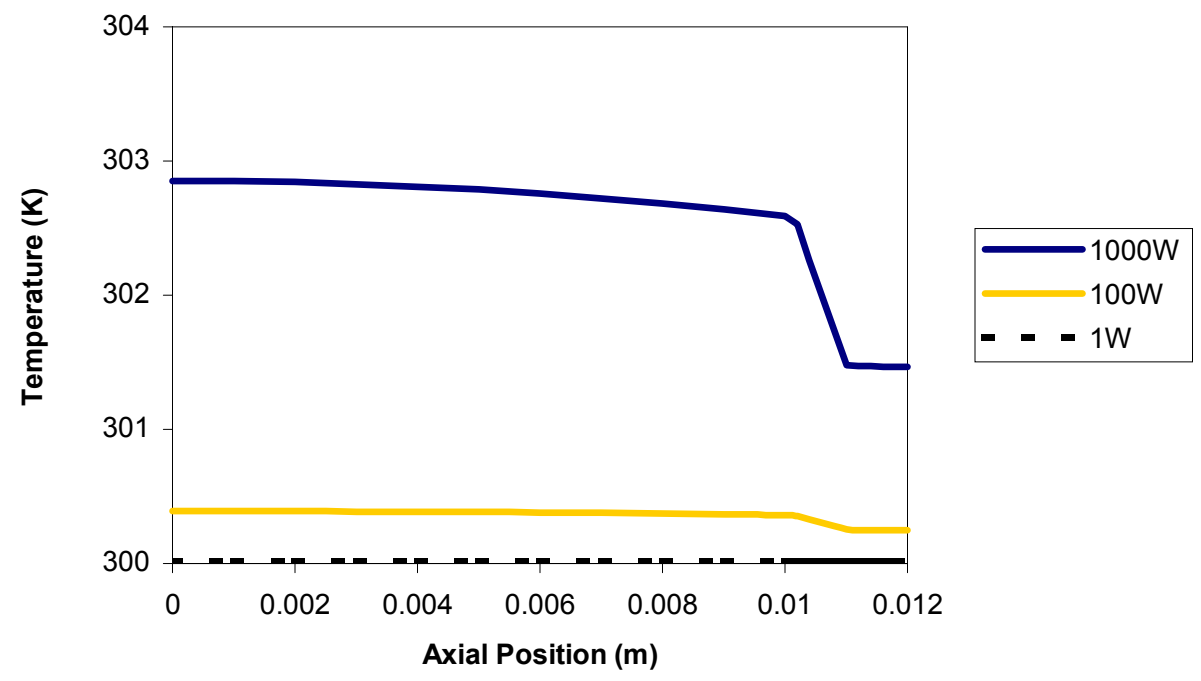

Figure 20 Axial temperature profiles at different SCM power levels

\section{Steady State Radial Heat Transfer}

This analysis models steady state conduction and external convection in the radial direction. This analysis also assumes that heat transfer between plates is negligible and that the surrounding cooling medium is effectively infinite and at constant temperature. Additional analysis should be performed to model heat transfer within an enclosure.

\section{Free Convection from an Isothermal Horizontal Cylinder}

Buoyancy effects can drive fluid flow around a heated cylinder, the SCM, which will be modeled as a long horizontal isothermal cylinder. The average heat transfer coefficient due to free convection can be determined as follows, $\bar{h}=\frac{k}{D} \bar{N} u_{D}$

where $D$ is the diameter of the cylinder. Churchill and Chu recommend the following Nusselt number correlation for long horizontal isothermal cylinders, ${ }^{\mathrm{xV}}$

$$
\bar{N} u_{D}=\left[0.60+\frac{0.387 R a_{D}^{1 / 6}}{\left[1+(0.559 / \mathrm{Pr})^{9 / 16}\right]^{3 / 27}}\right]^{2}
$$


for Rayleigh numbers, $R a_{D}$, less than approximately $10^{12}$ where the Rayleigh number is defined as,

$R a_{D}=\frac{g \beta\left(T_{s}-T_{\infty}\right) D^{3}}{v \alpha}$

with all material properties evaluated at $T_{\infty}$.

\section{Radial Conduction}

The calculation of radial conduction through the SCM is similar to the calculations performed for radial conduction through the CNS. However, in this case, the multiplier assembly is modeled as a central horizontal cylinder of $\mathrm{UO}_{2}$ with concentric annuli of Helium, Zircaloy, and lead. For the central cylinder with uniform heat generation, the temperature distribution is given by,

$$
T(r)=\frac{q^{\prime \prime \prime} r_{1}^{2}}{4 k}\left(1-\frac{r^{2}}{r_{1}^{2}}\right)+T_{1}
$$

The temperature difference between the outer lead surface and the surrounding medium is given by,

$T_{4}=T_{\infty}+\frac{\dot{q}}{2 h \pi r_{4} L}$

where $\dot{q}$ is the SCM power and $L$ is the length of the SCM. Temperature profiles within annuli are as before,

$T(r)=\frac{T_{1}-T_{2}}{\ln \frac{r_{1}}{r_{2}}} \ln \frac{r_{1}}{r_{2}}+T_{2}, \quad r_{1}<r<r_{2}$

with the temperature difference between the surfaces given by,

$T_{1}=T_{2}+\frac{\dot{q} \ln \frac{r_{2}}{r_{1}}}{2 \pi L k_{C u}}$

An iterative method similar to the method presented earlier was developed to perform this analysis for two coolants, water and air.

\section{Natural Convection to Water}

For natural convection in a large pool of water, temperatures in the SCM stay well below melting temperatures at 1000,100, 1, and $0.001 \mathrm{~W}$ of assembly power (see Figure 21). 


\section{Radial Conduction, Natural Convection in Water Radial Temperature Profile}

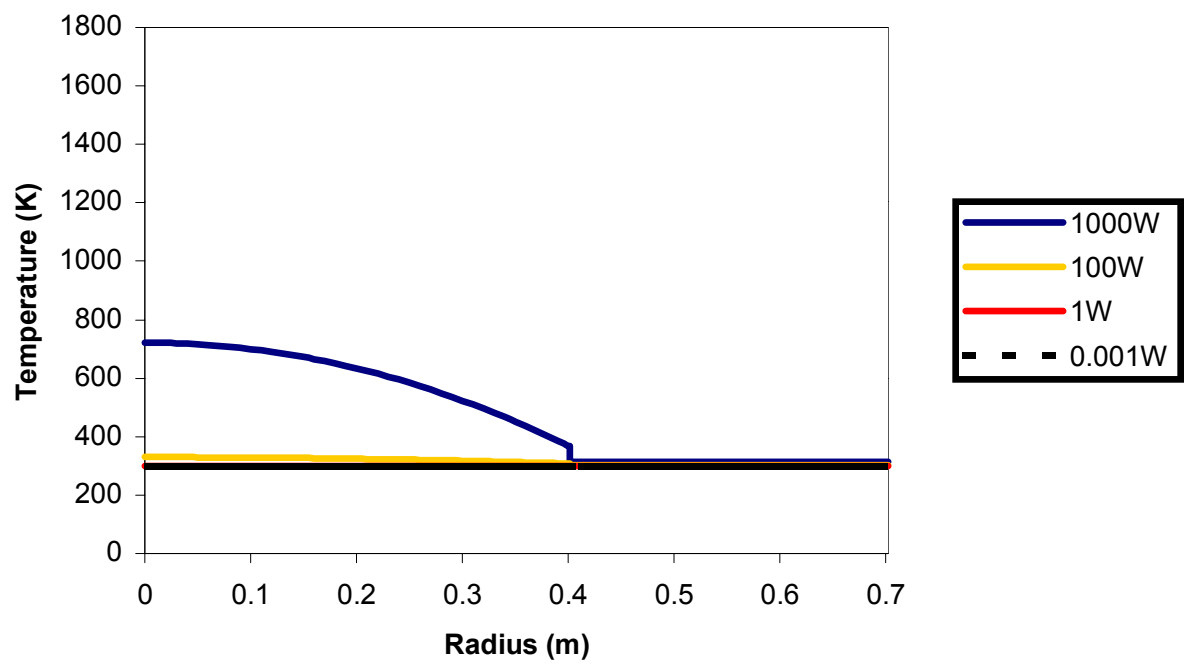

Figure 21 Radial temperature profiles for SCM in water.

\section{Natural Convection to Air}

However, in a loss of coolant accident (LOCA), it is conceivable that the water tank will be drained or perhaps all water has been boiled away (though this is unlikely given the relatively low power levels and high heat capacity of water). In response to such a scenario, analysis was performed for an SCM cooled by natural convection in air. In this case, the temperature of the lead annulus rises above the melting point for the extreme case of $1000 \mathrm{~W}$ of SCM power (See Figure 22). 


\section{Natural Convection in Air Without Fins \\ Radial Temperature Profile}

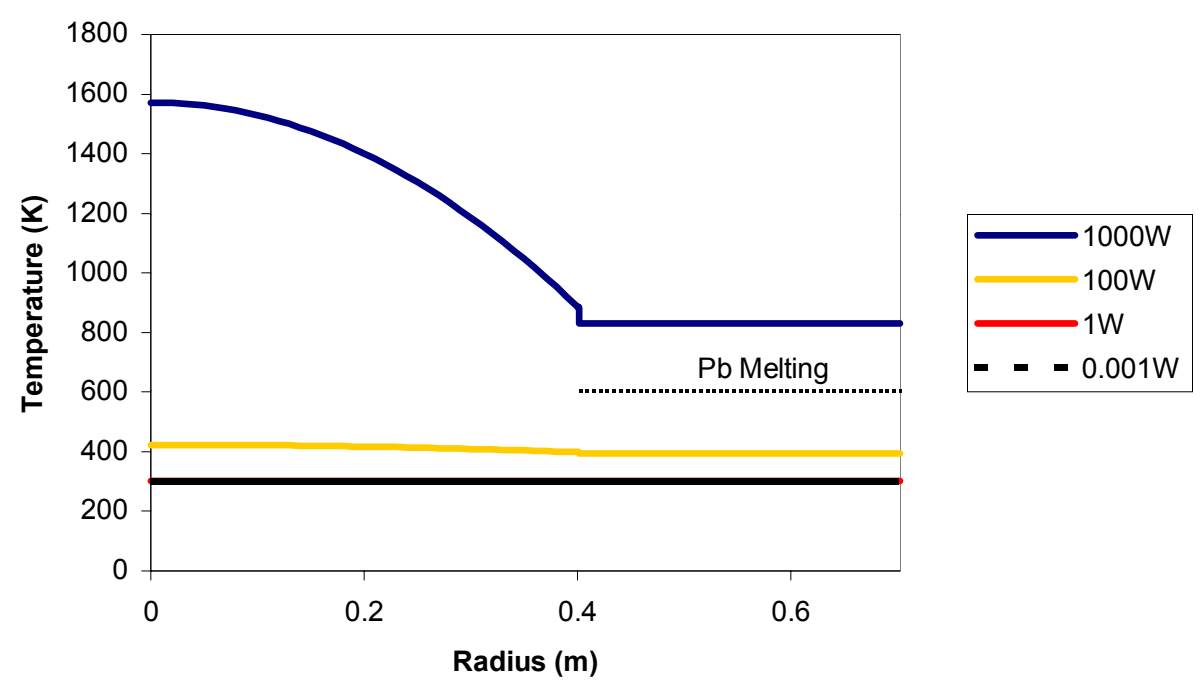

Figure 22 Radial temperature profiles for the SCM in air with $\mathrm{Pb}$ melting

\section{Annular Heat Fins}

While SCM power will be less than half of the extreme case in which lead melts, additional design work was carried out to address this potential hazard. Convective heat transfer can be enhanced by the addition of high conductivity heat sinks as shown schematically in Figure 23.

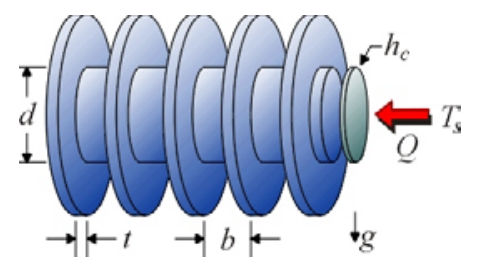

Figure 23 Schematic of annular fins

With the addition of these fins, the total heat transfer rate is given oy,

$\dot{q}=h A_{t}\left[1-\frac{N A_{f}}{A_{t}}\left(1-\eta_{f}\right)\right]\left(T_{\text {surface }}-T_{\infty}\right)$

with the fin area, $A_{f}$, defined as, $A_{f}=2 \pi\left(r_{2 c}^{2}-r_{1}^{2}\right)$

where $r_{1}$ is the inner radius of the fins (outer radius of SCM) and $r_{2 c}$ defined as the sum of the radius and half the thickness, $t$, of the fins; the total area, $A_{t}$, defined as, $A_{t}=N A_{f}+2 \pi r_{1}(H-N t)$

where $N$ is the number of fins and $H$ is the length of the SCM; and the fin efficiency, $\eta_{f}$. 
Incropera and DeWitt provide fin efficiencies in Figure 24 as a function of the ratio of $r_{2 c}$ to $r_{1}$ and the nondimensional number,

$L_{c}^{3 / 2}\left(h / k A_{p}\right)^{1 / 2}$

where the corrected fin length, $L_{c}$, is the sum of the actual fin length, $L$, and half of the fin thickness, $t$; and the corrected fin profile area, $A_{p}$, is the product of the corrected fin length and the fin thickness.

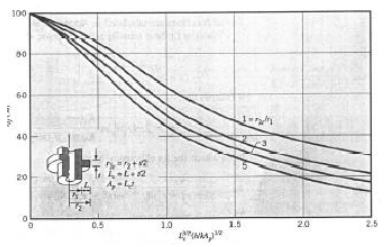

Figure 24 Efficiency of annular fins of rectangular profile. ${ }^{\text {xvi }}$

Using this analysis, five annular heat fins made out of 2024-T6 aluminum alloy were attached to outer surface of the lead annulus. Each of these fins is one centimeter in length and half a centimeter in thickness. Given that these fins are relatively spaced out along the SCM, it will be assumed that the convection heat transfer coefficient generated by free convection around the heated cylinder will not change.

Iterative methods were used again to determine the surface temperature and the resulting temperature profile at a variety of power levels (See Figure 25). As a result of the addition of annular heat fins, temperatures do not rise above melting and the SCM would be able to cool itself in air.

\section{Natural Convection in Air With Annular Fins \\ Radial Temperature Profile}

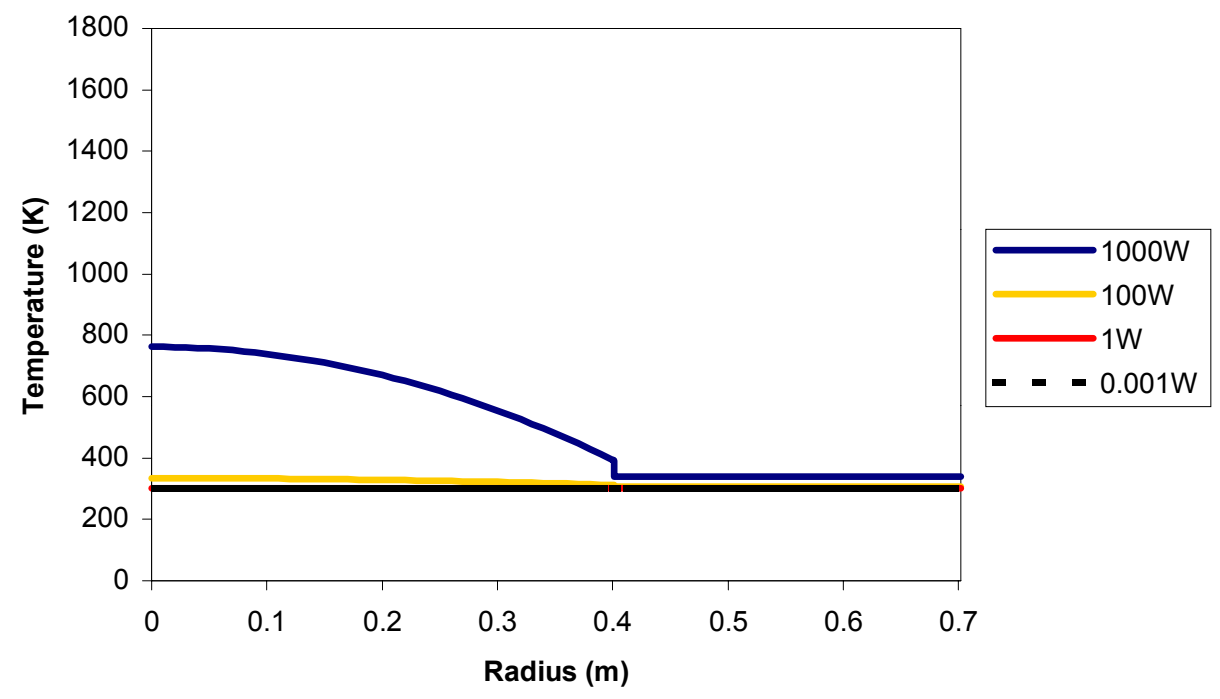

Figure 25 Radial temperature profiles for the SCM in air with annular heat fins. 


\section{Conclusions}

To amplify the neutrons from the CNS, a subcritical fission assembly consisting of Zircaloy cladding, uranium dioxide, light water, and a lead reflector was designed with MCNP. The design goal was a $\mathrm{k}_{\mathrm{eff}}$ of 0.98 , which corresponds to a multiplication factor of 50 . The moderatorto-fuel volume ratio was optimized to maximize $\mathrm{k}_{\infty}$. Varying the SCM dimensions and uranium enrichment maximized the neutron current into the Beam Shaping Assembly.

The optimal moderator-to-fuel volume ratio was found to be about 2.25:1. The optimal fission plate dimensions are $80 \mathrm{~cm}$ in diameter and about $14 \mathrm{~cm}$ in length with five $1 \mathrm{~cm}$ thick $3.8 \mathrm{w} / \mathrm{o}$ enriched plates. The $\mathrm{k}_{\mathrm{eff}}$ of this system is 0.983 resulting in about 30.4 neutrons leaking into the BSA per source neutron. The radial leakage of neutrons accounts for the discrepancy between the observed value and the expected multiplication factor of 50 .

Passive cooling mechanisms are sufficient to cool the SCM at all expected power levels in both normal and accident conditions. Complete two-dimensional modeling is desirable, but unnecessary to analyze this system. Temperature and fluid flow transients should also be modeled to ensure safe operation during startup or shutdown. However, given the large thermal inertia, high melting point, and low power density of the SCM, it is unlikely that any transients would develop that would challenge the safety of the system.

\section{Additional Considerations}

The neutronics of the subcritical multiplier assembly must be reanalyzed in response to the requirements for heat removal. First, the lithiated polyethylene layer cannot be in contact with the SCM due to its low conductivity and low melting temperature. A large water layer between the SCM and the polyethylene layer is ideal to provide cooling of the SCM via natural conduction and to provide cooling and neutron moderation between the fuel plates. Ducts must be cut out of the lead annulus to allow water to flow between the fuel plates, increasing neutron leakage through these channels. A container must be designed to contain the coolant such that leaks are unlikely to develop.

The addition of water poses additional requirements for radiation shielding due to the production of photons in water. An additional lining of lead could be used in conjunction with the polyethylene layer and the container structure to shield radiation. Similarly, radiation shielding must be addressed in the event of a loss of coolant accident.

\section{Goals}

\section{BEAM SHAPING ASSEMBLY (BSA)}

The compact BNCT apparatus uses two sources of neutrons to generate the intensities necessary for an effective treatment plan. The Compact Neutron Source produces isotropic mono-energetic neutrons through either D-D or D-T fusion reactions. The neutron source is then coupled to a Subcritical Multiplier (SCM) where a portion of the source neutrons will cause fissions, resulting in a source of fission spectrum neutrons. As a result, the neutron spectrum at the initial stages of 
the BSA will be a superposition of the source neutrons that have diffused and slowed though the SCM and partially thermalized fission neutrons from the SCM itself.

The most effective neutron energy range for BNCT is between 1 and $20 \mathrm{keV}{ }^{\text {xii }}$ The goal of the BSA is to efficiently moderate the source spectrum produced by fusion and fission reactions to the epithermal energy range between 1 to $20 \mathrm{keV}$ while maintaining a high enough intensity to result in a treatment time of about 1 hour.

\section{Assumptions}

Before integrated simulations were performed, it was assumed that the neutron spectrum at the interface between the SCM and BSA is essentially a Watt fission spectrum. This assumed spectrum was used to optimize the BSA before integrated MCNP runs were performed. The difference from the assumed spectrum and the spectrum resulting from simulation (Figure 26) is primarily due to the thermalization of neutrons in the SCM.

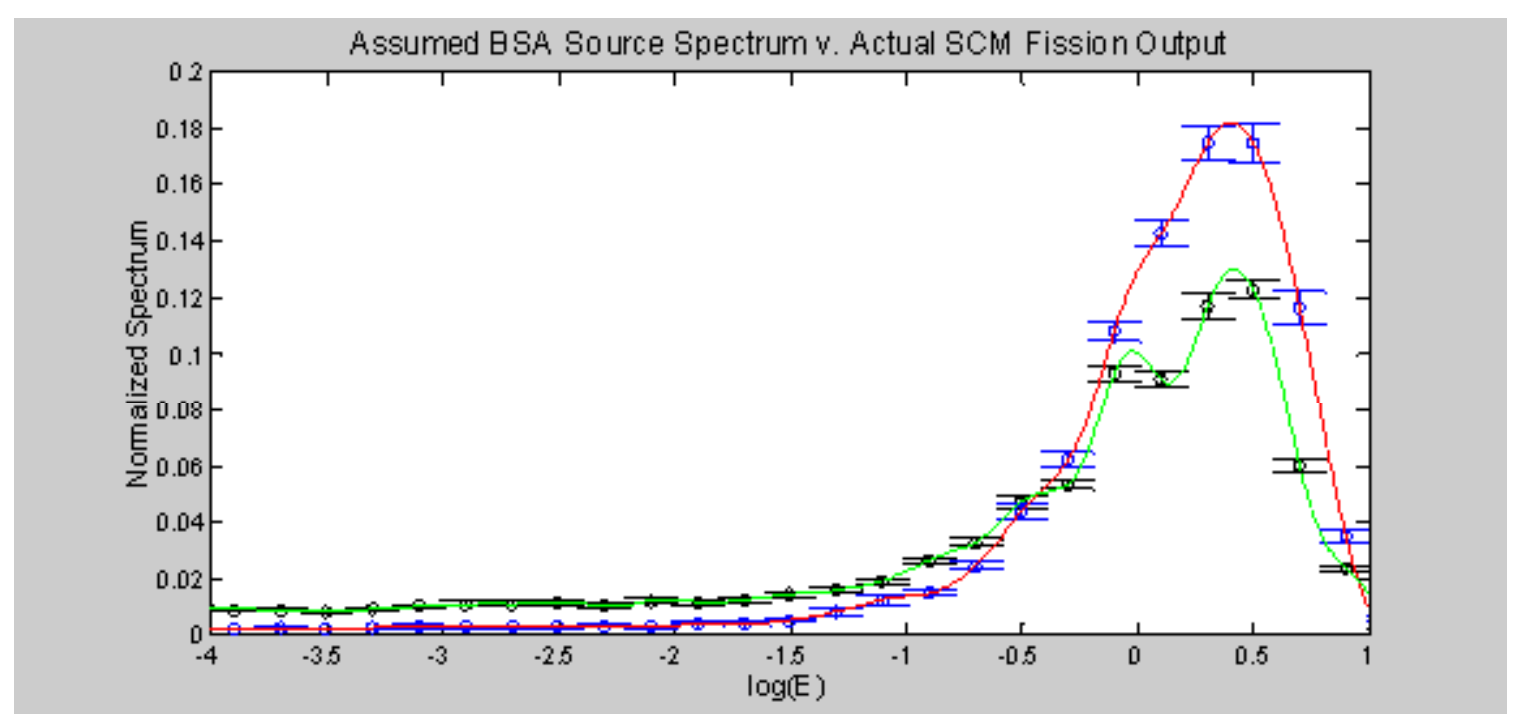

Figure 26 Assumed Fission Spectrum (red) as compared with actual spectrum of the subcritical fission multiplier output

\section{Previous Work}

Previous design work on beam shaping facilities has been carried out at the Fission Converter Facility at MIT's research reactor. In this design a filter/moderator of $\mathrm{Al}$ and Teflon $\left(\mathrm{CF}_{2}\right)$ is used to moderate fission neutrons from a $0.26 \mathrm{k}_{\text {eff }}$ fission converter assembly to a conical collimated output beam port. A lead shield is used to shield patients and personnel from gamma rays. Cadmium is used as a thermal neutron filter at the last stages of moderation. This reactorbased design yields approximately $10^{10}$ neutrons $/ \mathrm{cm}^{2} \mathrm{~s}$ of epithermal flux.

A previous study conducted at UC Berkeley of epithermal beam optimization produced results for neutrons at both 2.45 and $14 \mathrm{MeV}{ }^{\text {xviii }}$ In this study, different combinations of Lead, Iron, Al, and $\mathrm{AlF}_{3}$ are used to moderate the high-energy neutrons to between 1 and $40 \mathrm{keV}$. 
The spectrum from the SCM will most resemble the spectrum of the MIT Fission Converter Assembly given that both are based on fission. However, the study at Berkeley demonstrated the interesting qualities of Fluental, a composite material consisting of $40 \% \mathrm{Al}$ and $60 \% \mathrm{AlF}_{3}$, as a moderating material. Both studies used the resonance properties of Aluminum and Fluorine to reach a desired spectral and intensity profile.

\section{Fission Spectrum Moderation Study}

In this study, an assumed fission spectrum is used as a source of neutrons to emulate the SCM output as shown in Figure 26

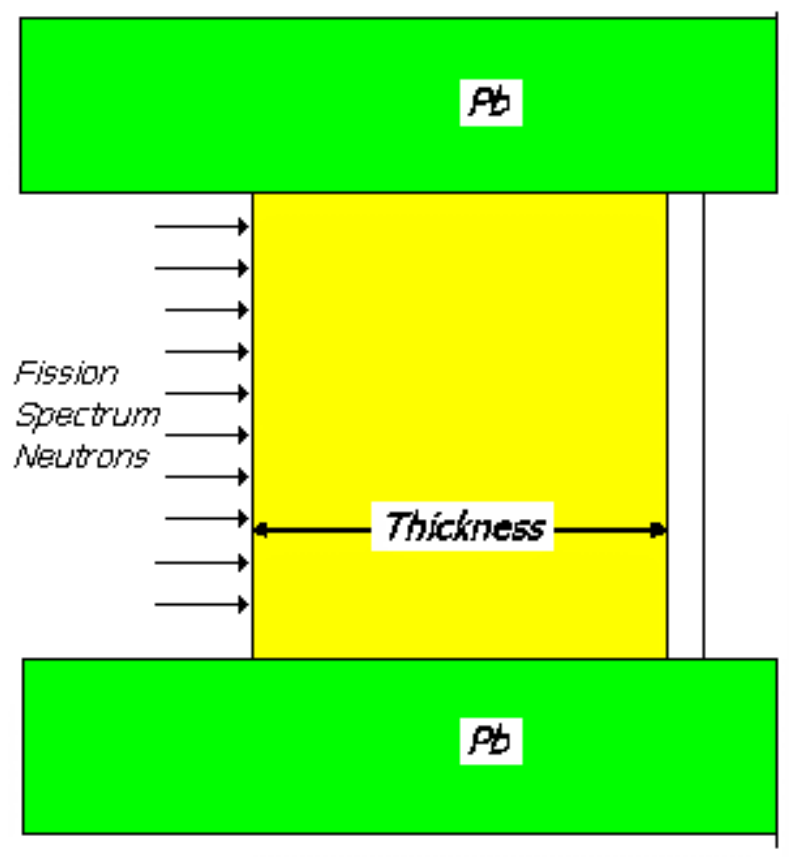

Figure 27 Fission Spectrum Study MCNP Model. The material is an $80 \mathrm{~cm}$ diameter cylinder with a $20 \mathrm{~cm}$ annulus of lead used for shielding. Monodirectional fission spectrum neutrons are applied and tallied in the right-most cell.

\section{Pb Study}

Using this MCNP model, the effects of different thicknesses of lead on the fission spectrum neutrons were studied. Being a very heavy element, lead will tend to shift the peak of the spectrum to lower energies without substantial amounts of thermalization. Minimizing the intensity of fast neutrons reduces harmful proton recoil reactions in healthy tissue.

Analyzing the cross-sections of the most common Pb isotopes (see Figure 28, Figure 29, and Figure 30 ), one notes that the absorption cross-sections are at least 2-3 orders of magnitude below the scattering cross-sections. In $\mathrm{Pb}-208$, which comprises $52 \%$ of natural $\mathrm{Pb}$, the 
absorption is even less pronounced. The effect is that lead will have good scattering properties with a neutron losing about $1 / 209$ or $0.5 \%$ of its initial energy after every collision.

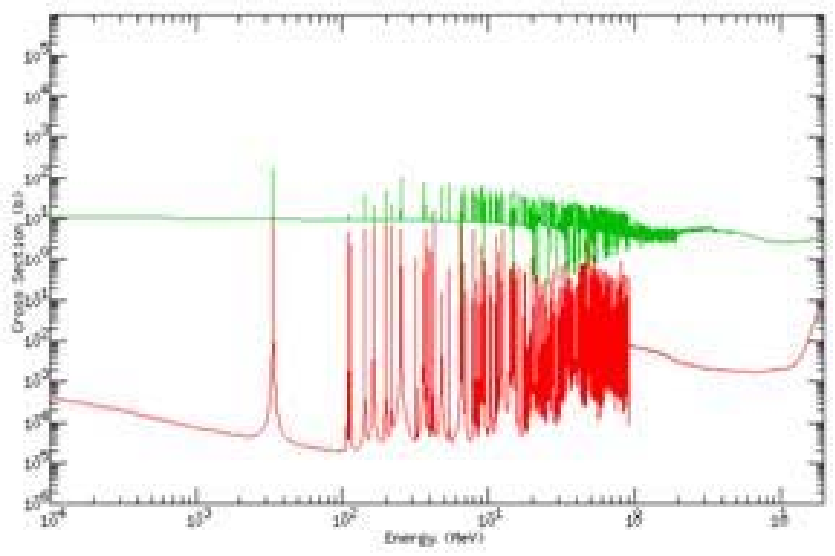

Figure 28 Elastic (green) and Absorption (red) microscopic cross-sections for Pb-206 from ENDF/B-6.0

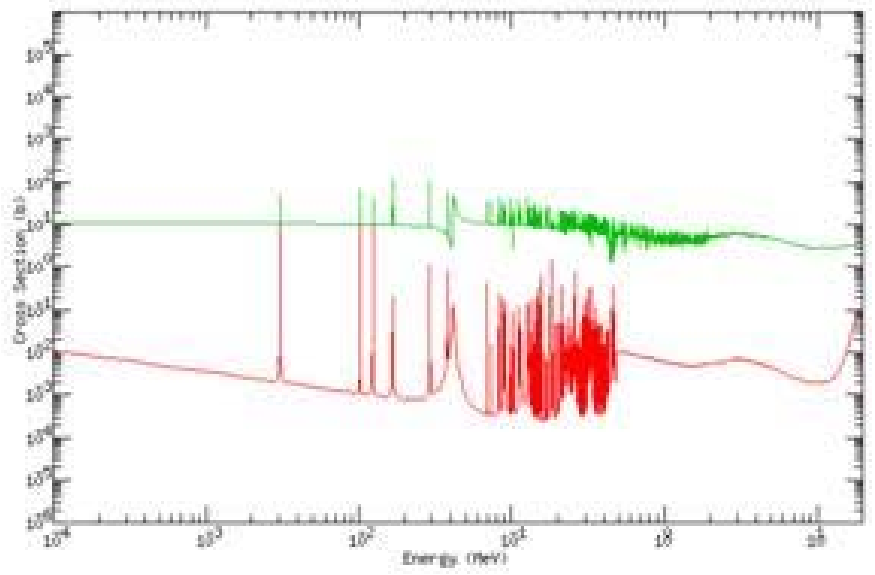

Figure 29 Elastic (green) and Absorption (red) microscopic cross-sections for Pb-207 from ENDF/B-6.0

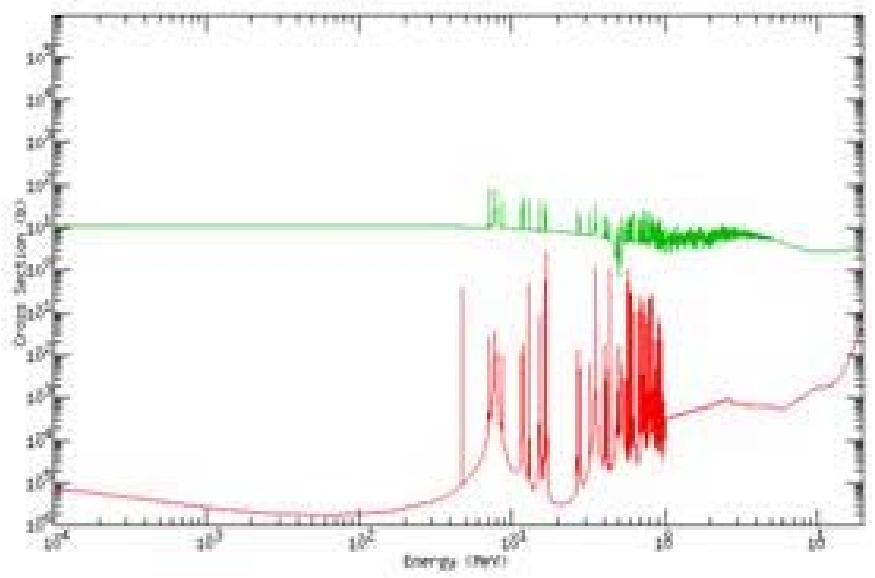

Figure 30 Elastic (green) and absorption (red) microscopic cross-sections for Pb-208 from ENDF/B-6.0

Using the MCNP model diagramed in Figure 27, the effects of varying the thickness of lead is determined by tallying the spectrum at the end opposite the assumed neutron source. As 
predicted, peak neutron energies shift to lower energies with increasing lead thickness with little thermalization (see Figure 31).

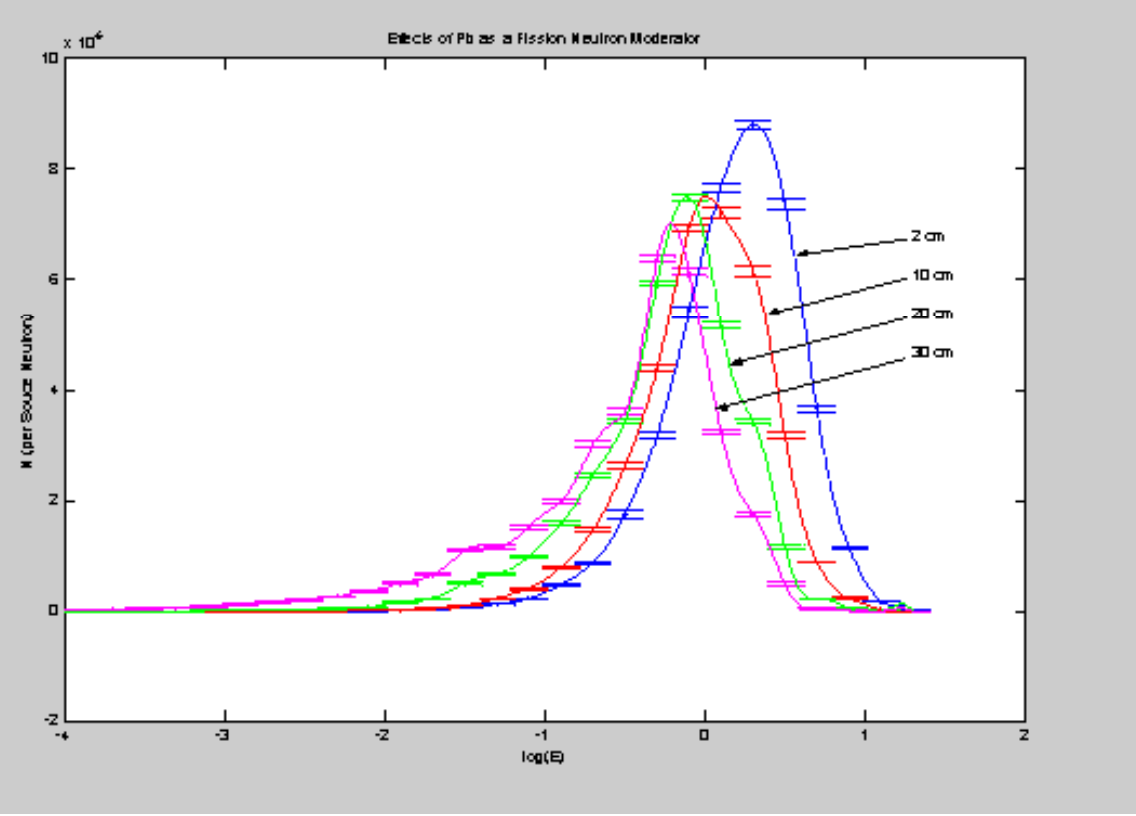

Figure 31 Effects of varying thickness of $\mathrm{Pb}$ moderator on a neutron fission spectrum

The fission spectrum is affected only by thick sections of $\mathrm{Pb}$ and the peak of the spectrum is shifted from about $2 \mathrm{MeV}$ with $2 \mathrm{~cm}$ of $\mathrm{Pb}$ to about $700 \mathrm{keV}$ with $30 \mathrm{~cm}$ of lead. This effect could be exploited to reduce fast neutrons to below $1 \mathrm{MeV}$ where other materials could possibly moderate the bulk of the fission neutrons more efficiently.

\section{Fe Study}

Iron is another heavy material with similar moderating ability. Iron is heavy enough to minimize the fraction of neutrons moderated to below $1 \mathrm{keV}$, but also has the cross section structure to possibly allow for the more efficient use of subsequent materials (see Figure 32 and Figure 33).

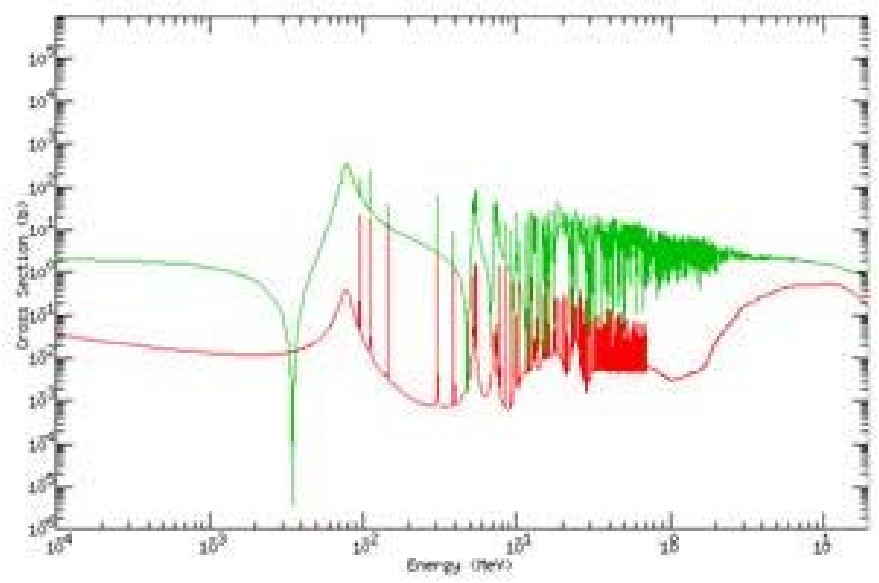

Figure 32 Elastic (green) and Absorption (red) microscopic cross-sections for Fe-54 (src:ENDF/B-6.0) 


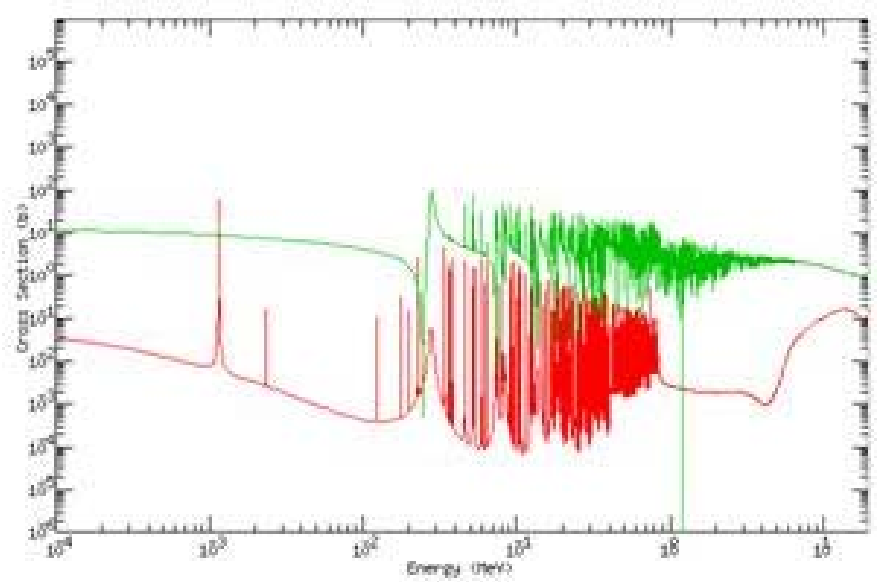

Figure 33 Elastic (green) and Absorption (red) microscopic cross-sections for Fe-56 (src:ENDF/B-6.0)

Naturally occurring iron contains approximately $92 \%$ Fe-56 so one would expect that the elastic and absorption peaks at $27 \mathrm{keV}$ would effect the spectrum significantly.

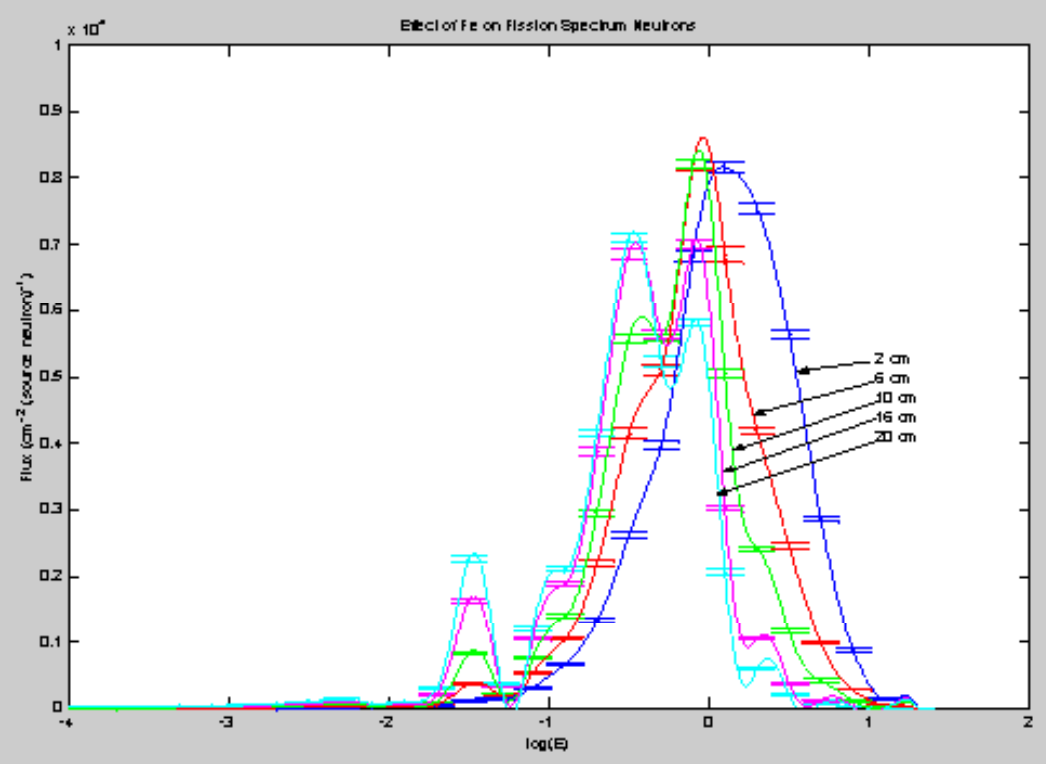

Figure 34 Results from varying thickness of Fe moderator on a neutron fission spectrum

Analysis of the effects of Fe on the fission spectrum using the MCNP model described earlier demonstrates the development of two peaks centered at about $40 \mathrm{keV}$ and $200 \mathrm{keV}$ (see Figure 34). These peaks are predominantly non-therapeutic as they increase the dose to healthy tissue with little impact on the tumor dose. The $40 \mathrm{keV}$ peak observed in the spectrum is the result of the $27 \mathrm{keV}$ peaks in the scattering cross-sections of iron. These peaks in the scattering crosssection act as a barrier to higher energy neutrons, resulting in a peak to the right (at higher energies) of the peak in the cross-section. 


\section{Using Resonance Peaks}

Understanding how the resonance peaks effect neutron spectra is important to effectively design the moderating material used in the BSA. In combination with the knowledge of the target spectra, one could find materials that have cross-sections that interfere with each other in energy ranges that are not preferred and have cross-sections that constructively add in energy ranges that are preferred. A material which exhibits this complimentary superposition of cross-sections is Fluental, an alloy composed of $40 \%$ Aluminum and $60 \% \mathrm{AlF}_{3}$.

\section{$40 \% \mathrm{Al} / 60 \% \mathrm{AlF}_{3}$ Study}

Analysis of this material shows a coupling of cross sections that produces a destructive effect at most energies greater that $40 \mathrm{keV}$ and a constructive effect at energies in the therapeutic range of 1 to $30 \mathrm{keV}$ (see Figure 35).
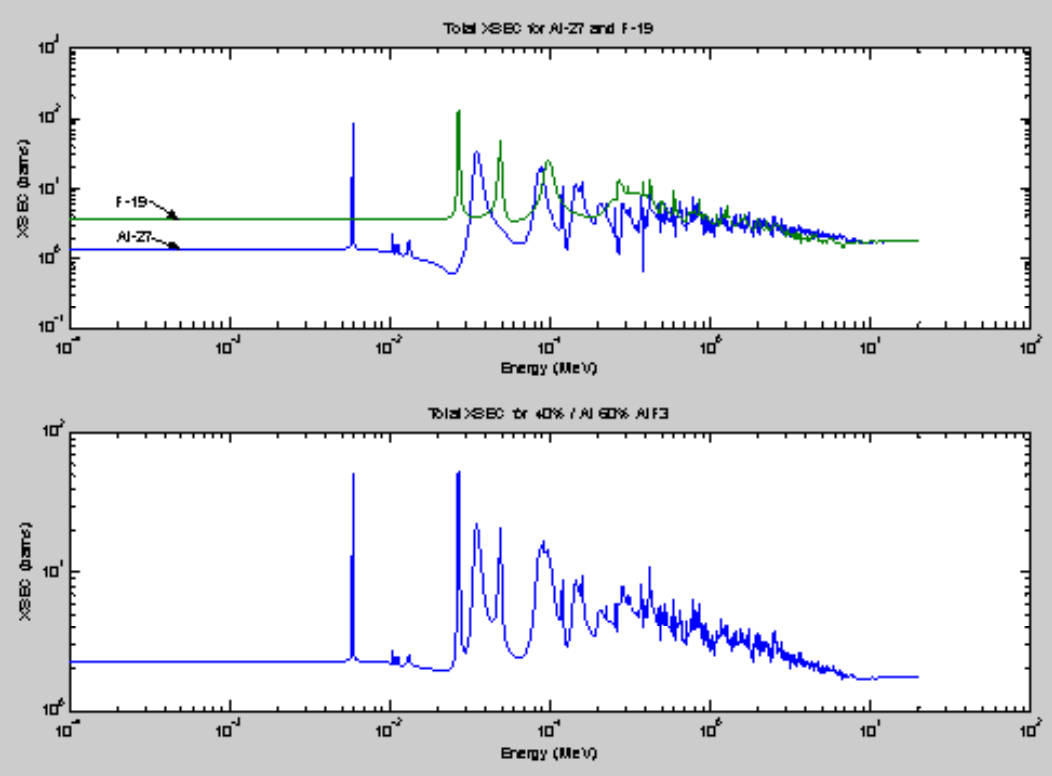

Figure 35 (a) Top: Total cross-sections for F-19 and Al-27 showing the destructive effect of resonances at most energies (b) Bottom: Combined microscopic cross section for mixture of $\mathrm{Al} / \mathrm{AlF}_{3}$ showing constructive effects at ranges of $7-30 \mathrm{keV}, 70 \mathrm{keV}$, and $700 \mathrm{keV}$

The cross sections of Aluminum and Fluorine show both destructive and constructive effects. For the most part, the cross sections allow fast neutrons above $1 \mathrm{MeV}$ to slow to the desired epithermal ranges. However, there are two regions of concern. First, the resonance peak at 170 $\mathrm{keV}$ acts as a barrier, creating a peak at an energy higher than the cross-section peak. Second, a $70 \mathrm{keV}$ peak in the neutron spectrum is expected as result of the combination of the large resonances between 25 and $60 \mathrm{keV}$ and the cross-section "valley" between 60 and $80 \mathrm{keV}$. 


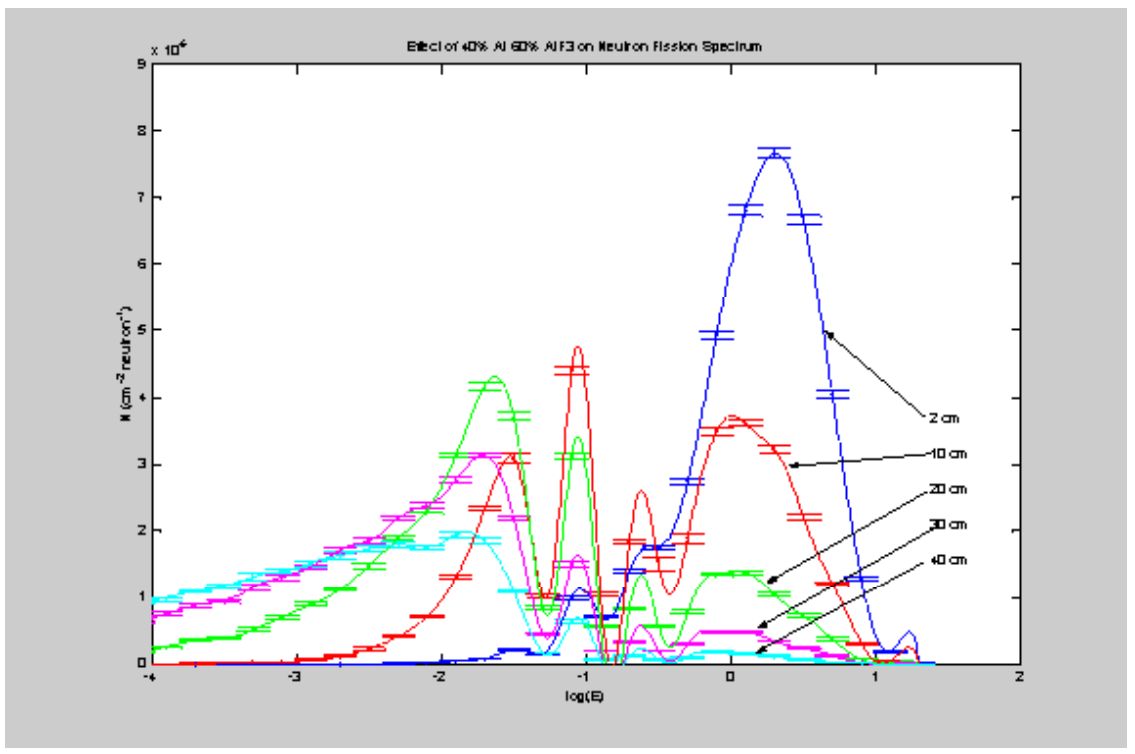

Figure 36 Effects of varying thickness of $40 \% \mathrm{Al} 60 \% \mathrm{AlF} 3$ on a neutron fission spectrum

Analysis of how the fission spectrum is moderated by the Aluminum/Fluorine mixture shows the development of three peaks that recede with increasing Fluental thickness. The peaks at 200 and $70 \mathrm{keV}$ develop first and peak with about $10 \mathrm{~cm}$ of moderator. The epithermal peak at $20 \mathrm{keV}$ develops later and reaches a maximum with about $20 \mathrm{~cm}$ of moderator. It is this epithermal 20 $\mathrm{keV}$ peak that is of interest due to its therapeutic value (see Figure 36).

\section{${ }^{7}$ LiF Study}

Lithium Fluoride is a light material and therefore will be placed in later stages of the BSA to filter the fast portion of the neutron spectrum to reduce the dose to healthy tissue. Analyzing the cross-sections, both Lithium and Fluorine have relatively small probabilities for absorption with the exception of fast neutrons above $3 \mathrm{MeV}$. Resonance peaks at 20, 40 and $90 \mathrm{keV}$ for Lithium will cause some peaking of the neutron spectrum in these regions (see Figure 37 ).

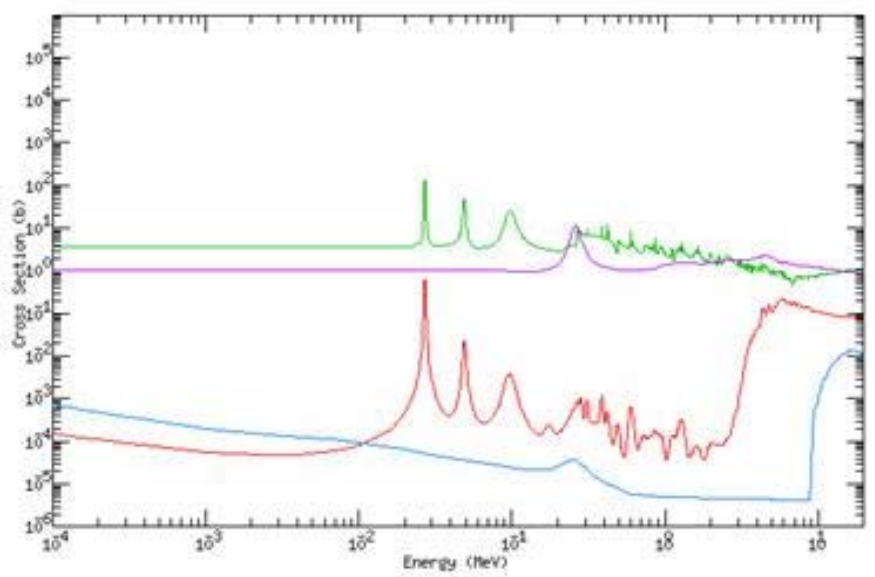

Figure 37 7Li elastic scattering (purple) and absorption (blue) microscopic cross-sections and 19F elastic (green) and absorption (red) microscopic cross-sections (src:ENDF/B-6.0) 


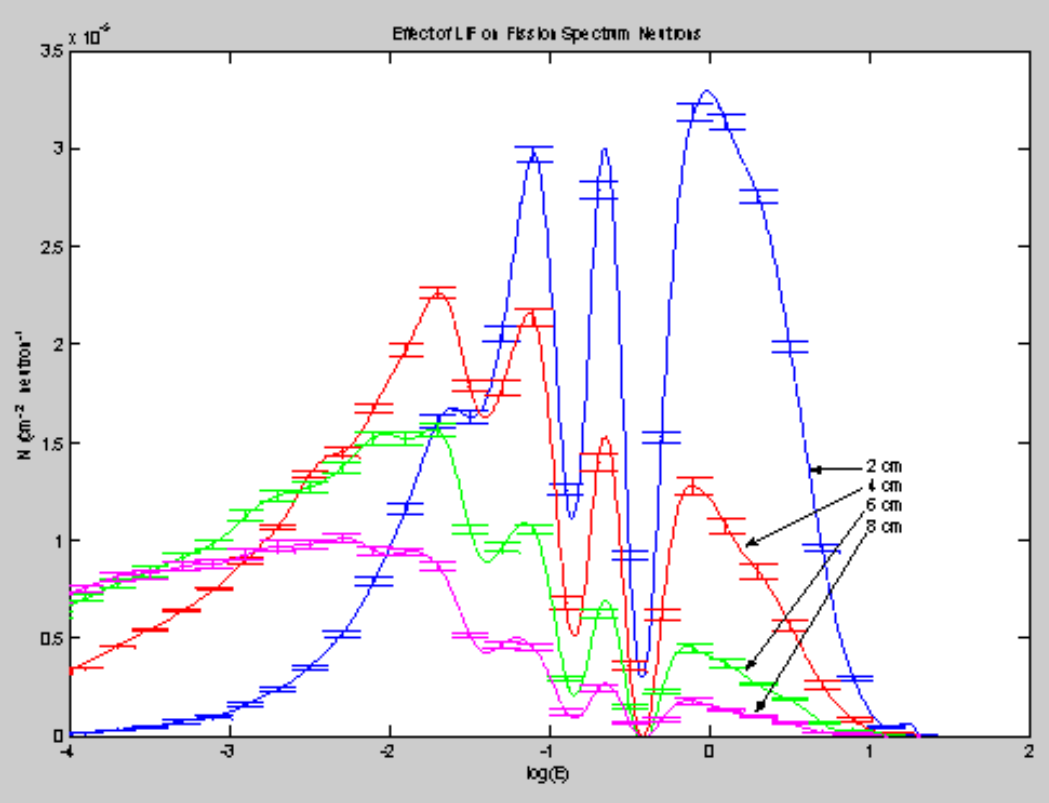

Figure 38 Effect of varying thickness of ${ }^{7} \mathrm{LiF}$ on Fission Spectrum Neutrons

The results of the LiF study shows that the fission spectrum peak can be shifted with as little as 8 $\mathrm{cm}$ of material. An interesting observation is that between $2 \mathrm{~cm}$ and $8 \mathrm{~cm}$ of LiF, the $70-80 \mathrm{keV}$ peak is reduced to around $15 \%$ of the original intensity while the peak at $12 \mathrm{keV}$ is reduced only to only $41 \%$ of original intensity. In addition, the $12 \mathrm{keV}$ peak is shifted to about $7 \mathrm{keV}$, an energy with higher therapeutic value.

\section{Optimized Material Combination}

Based on this analysis, a combination of Aluminum and Fluorine was determined to be the optimum material for moderating and filtering a fission neutron spectrum. To arrive at the optimum thickness of material, the neutron intensity at energies around $10 \mathrm{keV}$ should be maximized while fast neutrons above $40 \mathrm{keV}$ should be minimized to maximize the ratio between tumor healthy tissue dose. To visualize this process, a flux intensity map was created to plot neutron intensity as a function of moderator thickness and energy. 


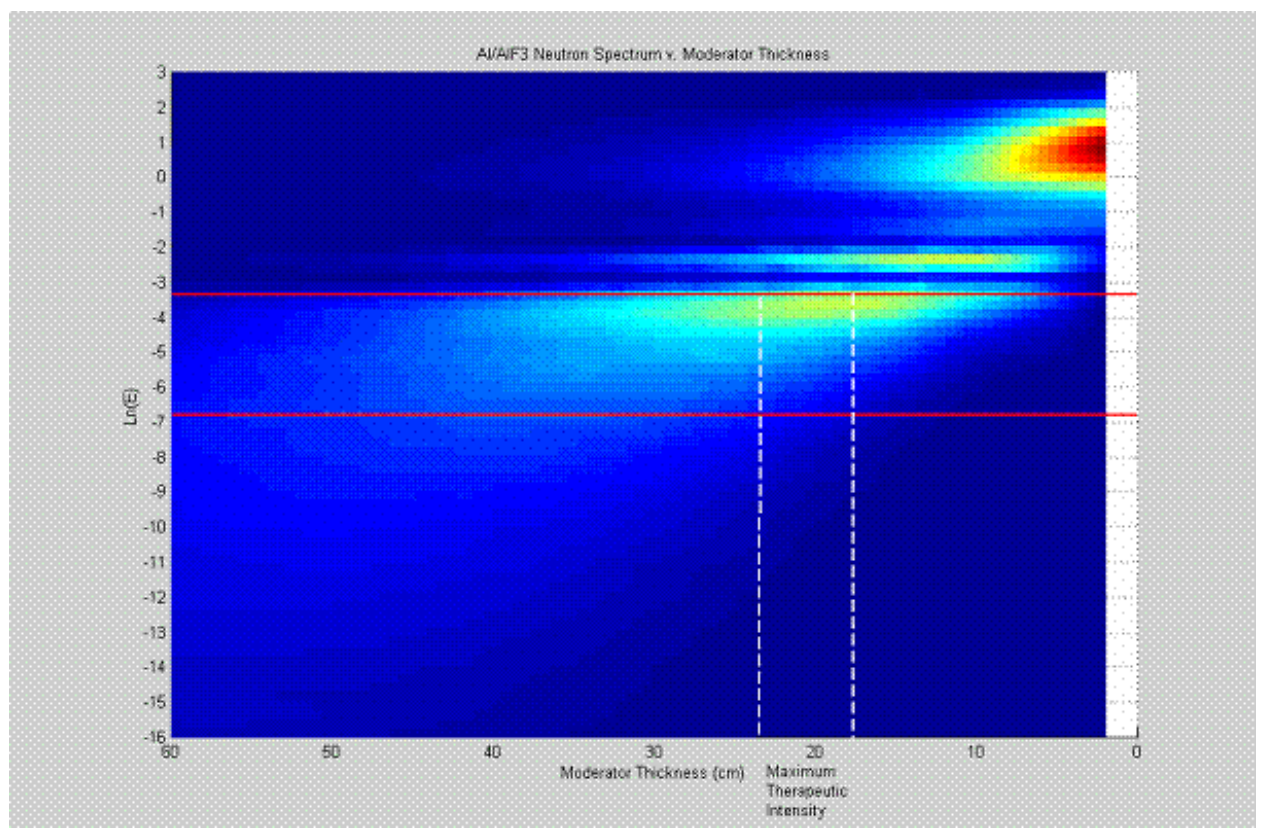

Figure 39 Neutron flux intensity as a function of energy and moderator thickness

Although the maximum of the therapeutic region $(1-40 \mathrm{keV})$ occurs with about $20 \mathrm{~cm}$ of Fluental, the fast spectrum is much too high and would result in an unacceptable dose to healthy tissue. Examining the plot, $45 \mathrm{~cm}$ of Fluental was determined to minimize the fast spectrum while maximizing the intensities in the therapeutic region (see Figure 39).

\section{${ }^{6}$ LiF Study}

In prior literature, it was found that ${ }^{6} \mathrm{Li}$ is an efficient filter of thermal neutrons, as evidenced by its increasing absorption cross section at thermal energies (see Figure 40).

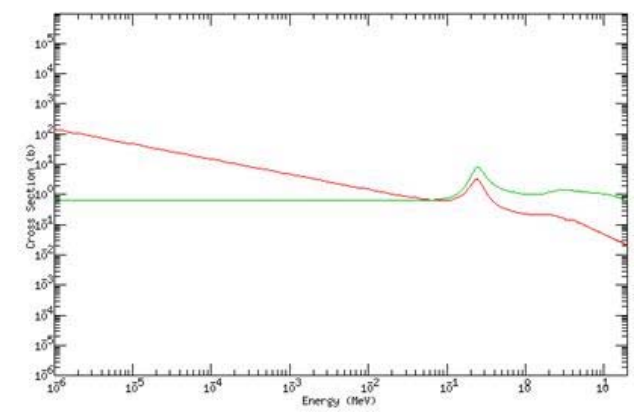

Figure 40 Elastic (green) and absorption (red) cross sections of ${ }^{6} \mathrm{Li}$ (src:ENDF/B-6.0)

The effects of inserting a thin $(0.1-0.5 \mathrm{~cm})$ layer of ${ }^{6} \mathrm{LiF}$ after the Fluental moderator were studied to try to reduce the intensities of thermal neutrons while maintaining epithermal neutrons of therapeutic value. 


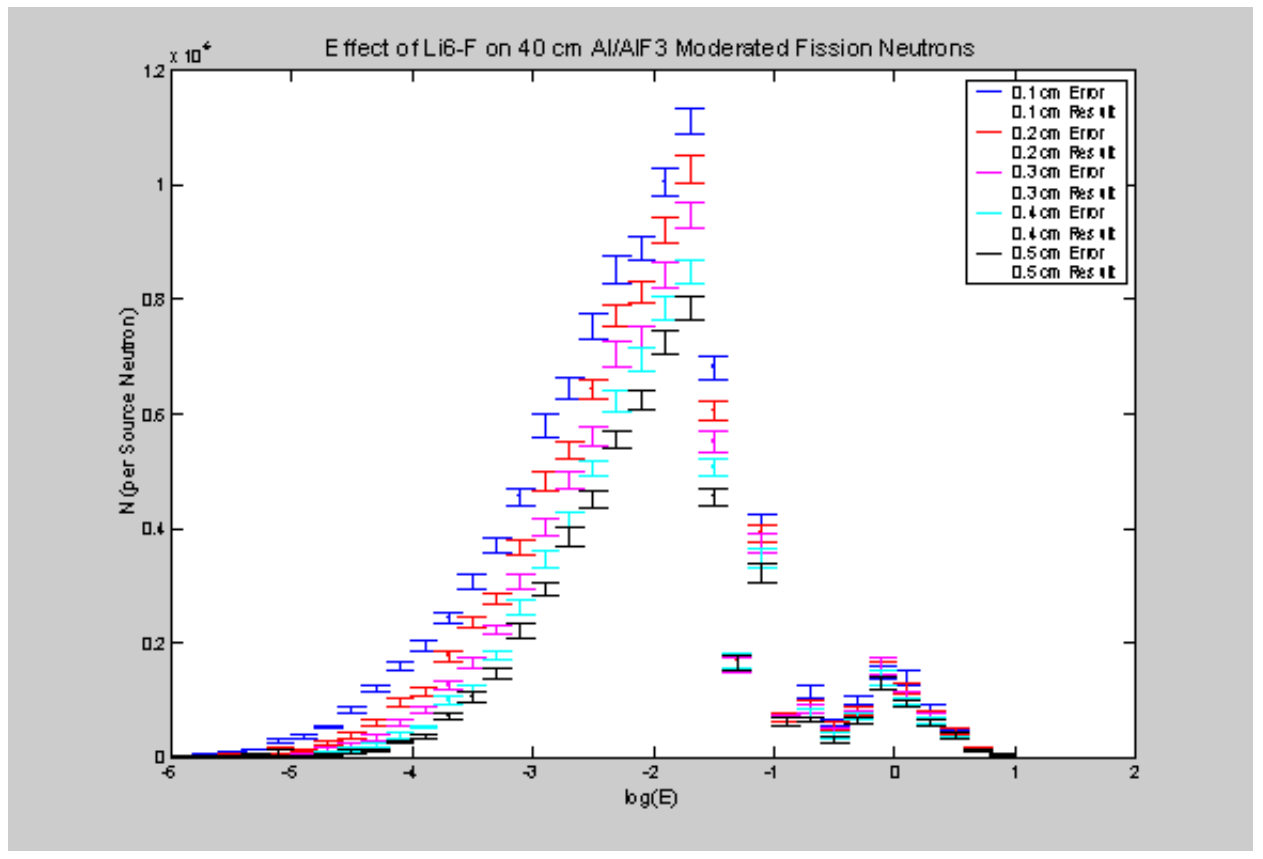

Figure 41 Effects of ${ }^{6} \mathrm{LiF}$ filter after $40 \mathrm{~cm}$ Fluental moderator

From the simulations, the effects of ${ }^{6} \mathrm{LiF}$ are quite pronounced. As expected, more thermal neutrons are absorbed as the thickness of ${ }^{6} \mathrm{LiF}$ increases. Though a $0.5 \mathrm{~cm}$ thick layer absorbed more thermal neutrons than a $0.1 \mathrm{~cm}$ thick layer, the thicker layer also decreased the intensity of epithermal neutrons. Unfortunately, thermal neutrons do not penetrate tissue as well as epithermal neutrons, reducing the ability of the beam to treat tumors located deep within the brain. As a result, the thinner $0.1 \mathrm{~cm}$ layer of ${ }^{6} \mathrm{LiF}$ was chosen to maintain a higher intensity of epithermal neutrons.

\section{Assembly Diameter Study}

In previous studies, the diameter of the moderating assembly was analyzed. In the Berkeley study using monoenergetic neutron beams, the diameter of a cylindrical BSA was optimized at $80 \mathrm{~cm}^{\text {xix }}$. To validate this result, a comparison was performed with $80 \mathrm{~cm}$ and $50 \mathrm{~cm}$ diameters to observe the effects on the output spectrum. The model for this study was a cylindrical BSA with $40 \mathrm{~cm}$ of Al/AlF3 and $0.1 \mathrm{~cm}$ of ${ }^{6} \mathrm{LiF}$ surrounded by a $30 \mathrm{~cm}$ thick lead annulus for neutron reflection and shielding. Based on the Berkeley design, the output collimator, a conical port with diameters of $20 \mathrm{~cm}$ and $12 \mathrm{~cm}$, was hollowed out of a $12 \mathrm{~cm}$ thick lithiated polyethylene neutron shield placed at the business end of the BSA. A fission spectrum source was introduced at the left of the moderating material and a flux tally was performed at the output window of the assembly (see Figure 42). 


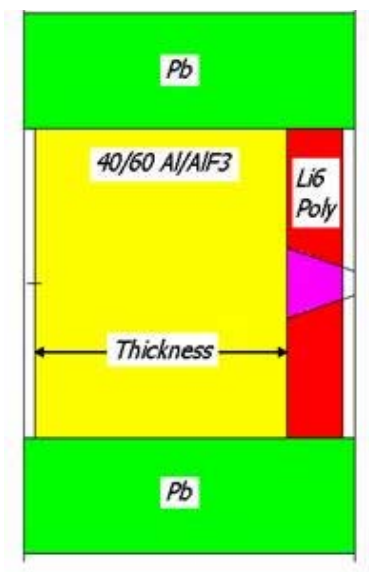

Figure 42 Model for assembly diameter study

The results indicate that assembly diameter has little effect on the width of the epithermal spectrum. While the $80 \mathrm{~cm}$ diameter BSA had higher intensities, the ratio between fast and epithermal neutrons did not change significantly with different BSA diameters (see Figure 43). As a result, an $80 \mathrm{~cm}$ assembly diameter was selected to increase the efficiency of the subcritical multiplier (see previous discussion on SCM optimization) and to increase neutron intensity at the output window.

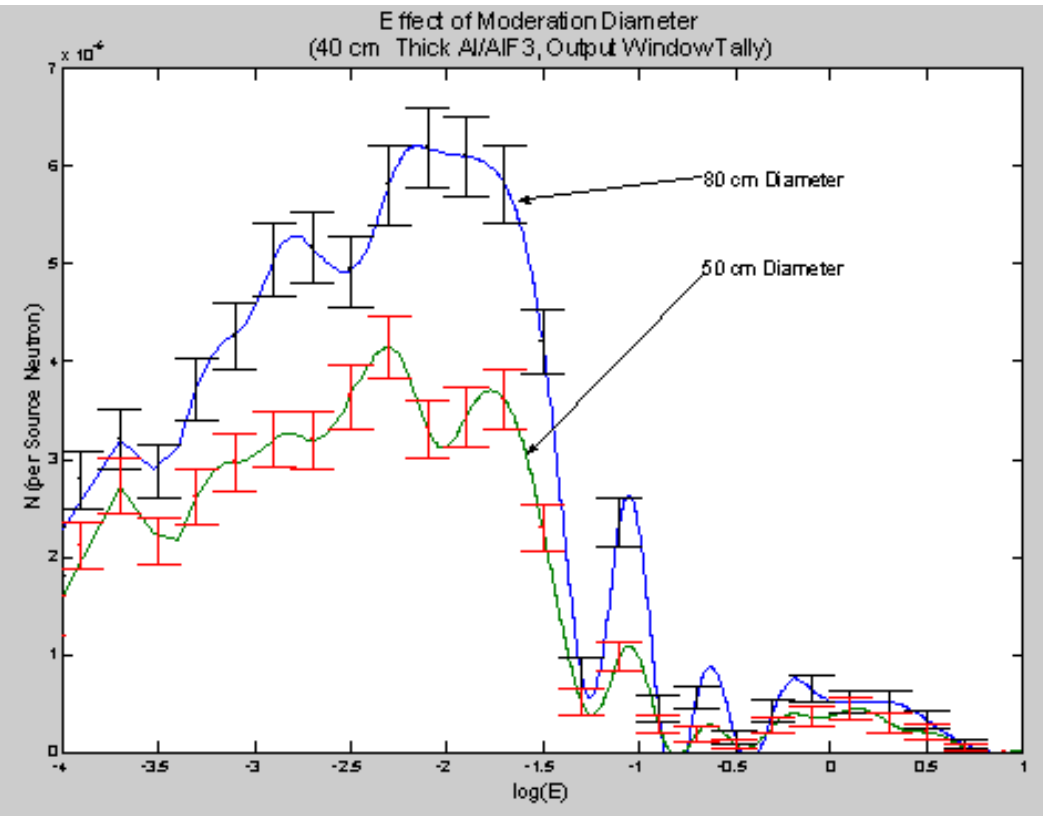

Figure 43 Results of assembly diameter study

\section{Conclusions}

Using a fission spectrum assumption as the input spectrum to the BSA, conclusions can be drawn as to the effects of different materials with respect to the moderating capabilities. It remains that an optimum combination of Aluminum and Fluorine will create an epithermal peak at near optimum energy for BNCT. The mixture of $40 \% \mathrm{Al} / 60 \% \mathrm{AlF}_{3}$ was analyzed to be 
optimum at around $45 \mathrm{~cm}$ thickness in an $80 \mathrm{~cm}$ cylindrical geometry. The effect of diameter of the assembly was that the intensity was diminished with a smaller diameter vice a larger one. A $0.1 \mathrm{~cm}$ thick ${ }^{6} \mathrm{LiF}$ layer was used a thermal neutron filter.

\section{FACILITY INTEGRATION \& DOSE}

Upon performing the design iterations on each segment of the facility, an integrated MCNP model was developed to simulate the performance of the design from source neutron to patient. Unfortunately, the computational time required to produce meaningful data from this complex geometry was unavailable. While preliminary data exists, large statistical errors obfuscate the data. As a result, patient dose and treatment times from this device remain inadequately quantified. Variance reduction techniques in addition to longer simulations will likely be required to improve the statistics.

However, Verbeke's work with D-D fusion Compact Neutron Sources (CNS) coupled to a Beam Shaping Assembly (BSA) resulted in treatment times of approximately 30 hours. ${ }^{\mathrm{xx}}$ Given the neutron multiplication factor of 30 in the Subcritical Multiplier (SCM), it seems likely that a treatment time of one hour can be achieved with this facility.

\section{REFERENCES}

i ... "General information about Brain Cancer." July 29, 2002,

$<\mathrm{http}: / /$ www.holisticcancersolutions.com/BraintumorGeneralreport.htm>.

ii Verbeke, Jerome. "Development of High-Intensity D-D and D-T Neutron Sources and Neutron Filters for Medical and Industrial Applications." Diss. University of California, Berkeley. 2000.

iii ... "General information about Brain Cancer." July 29, 2002,

$<\mathrm{http}$ //www.holisticcancersolutions.com/BraintumorGeneralreport.htm>.

iv See Endnote ii

${ }^{v}$ See Endnote ii

${ }^{v i}$ Leung, Ka Ngo. Professor in Residence. University of California at Berkeley/Lawrence Berkeley National Laboratory. Personal Communication.

vii Stevenson. "Introduction to Nuclear Engineering." 1958.

viii See Endnote vi

${ }^{\text {ix }}$ M. S. Bhatti and R. K. Shah, Turbulent and Transition Flow Convective Heat Transfer in Ducts, Handbook of Single-Phase Convective Heat Transfer, John Wiley and Sons, 1987.

${ }^{\mathrm{x}}$ W. M. Kays and E. Y. Leung, Heat Transfer in Annular Passages: Hydrodynamically Developed Turbulent Flow with Arbitrarily Prescribed Heat Flux, International Journal of Heat and Mass Transfer, Vol. 6, pp. 537-557, 1963.

${ }^{\mathrm{xi}}$ See Endnote ii 
xii Bar-Cohen, A. and W.M. Rohsenow, "Thermally Optimum Spacing of Vertical Natural Convection Cooled Parallel Plates," Journal of Heat Transfer, pp. 106-116, 1984.

xiii United States. Argonne National Laboratory: International Nuclear Safety Center. Thermal Conductivity and Thermal Diffusivity of Solid UO2, <http://www.insc.anl.gov/matprop/uo2/cond/thcsuo2.pdf $>$.

xiv United States. Argonne National Laboratory: International Nuclear Safety Center. Zircaloy Thermal Conductivity: Preliminary Recommendation, $<$ http://www.insc.anl.gov/matprop/zircaloy/zirck.pdf $>$.

${ }^{x v}$ Churchill, S. W., and H. H. S. Chu, "Correlating Equations for Laminar and Turbulent Free Convection from a Horizontal Cylinder," International Journal of Heat and Mass Transfer, 18, 1049, 1975.

${ }^{\text {xvi }}$ Incropera, F. P. and D. P. DeWitt. Fundamentals of Heat and Mass Transfer. John Wiley \& Sons, Inc. p. 123, 1996.

${ }^{\text {xvii }}$ See Endnote ii

xviii See Endnote ii

${ }^{\text {xix }}$ See Endnote ii

${ }^{\mathrm{xx}}$ See Endnote ii 